\title{
The bear in Eurasian plant names: motivations and models
}

\author{
Valeria Kolosova ${ }^{1 *}$, Ingvar Svanberg ${ }^{2}$, Raivo Kalle ${ }^{3}$, Lisa Strecker ${ }^{4}$, Ayşe Mine Gençler Özkan ${ }^{5}$, Andrea Pieroni ${ }^{6}$, \\ Kevin Cianfaglione ${ }^{7}$, Zsolt Molnár $^{8}$, Nora Papp ${ }^{9}$, Łukasz Łuczaj $^{10}$, Dessislava Dimitrova ${ }^{11}$, Daiva Šeškauskaite ${ }^{12}$, \\ Jonathan Roper ${ }^{13}$, Avni Hajdari ${ }^{14}$ and Renata Sõukand ${ }^{3}$
}

\begin{abstract}
Ethnolinguistic studies are important for understanding an ethnic group's ideas on the world, expressed in its language. Comparing corresponding aspects of such knowledge might help clarify problems of origin for certain concepts and words, e.g. whether they form common heritage, have an independent origin, are borrowings, or calques. The current study was conducted on the material in Slavonic, Baltic, Germanic, Romance, Finno-Ugrian, Turkic and Albanian languages. The bear was chosen as being a large, dangerous animal, important in traditional culture, whose name is widely reflected in folk plant names. The phytonyms for comparison were mostly obtained from dictionaries and other publications, and supplemented with data from databases, the co-authors' field data, and archival sources (dialect and folklore materials). More than 1200 phytonym use records (combinations of a local name and a meaning) for 364 plant and fungal taxa were recorded to help find out the reasoning behind bear-nomination in various languages, as well as differences and similarities between the patterns among them. Among the most common taxa with bear-related phytonyms were Arctostaphylos uva-ursi (L.) Spreng., Heracleum sphondylium L., Acanthus mollis L., and Allium ursinum L., with Latin loan translation contributing a high proportion of the phytonyms. Some plants have many and various bear-related phytonyms, while others have only one or two bear names. Features like form and/or surface generated the richest pool of names, while such features as colour seemed to provoke rather few associations with bears. The unevenness of bear phytonyms in the chosen languages was not related to the size of the language nor the present occurence of the Brown Bear in the region. However, this may, at least to certain extent, be related to the amount of the historical ethnolinguistic research done on the selected languages.
\end{abstract}

Keywords: Ethnobotany, Ethnolinguistics, Traditional knowledge, Phytonyms, Brown bear Ursus arctos, Motivation, Latin calques

\section{Background}

Many plant names have an animal element as a part of them. Animal names in folk (and scientific) plant names are common in many languages in Europe, as well as outside Europe. The prefix bear- is very common in many languages in the Eurasian area, which reflects the bear's importance in the folk tradition.

Phytonyms (that is plant names) with animal names as components have been discussed by many ethnobotanists, folklorists, and linguists. Already a pioneering

\footnotetext{
*Correspondence: chakra@eu.spb.ru

'Institute for Linguistic Studies, Russian Academy of Sciences, Tuchkov pereulok 9, Saint-Petersburg 199053, Russia

Full list of author information is available at the end of the article
}

plant name researcher $\mathrm{T}$. Thiselton-Dyer [1] observed that bear was a common animal element in many plant names, giving bear's foot, bear-berry, bear's bilberry, bear's-garlic, bears-breech, and bear's-wort as examples. The word bear could denote the size, the coarseness, and frequently the worthlessness or spuriousness of the plant. H. Kreiter [2] gave an account of French vernacular names of plants derived from animal names. $\mathrm{H}$. Marzell published a dissertation on animals in German plant names [3]. Croatian and general Slavonic material was analysed in works by N. Vajs [4] and S. Dubrovina [5], respectively. I. Hauenschield provided an important contribution with her studies on the use of animals in Turkic plant names [6]. She noted that the bear is an 
important animal in Turkish folklore and also has a very prominent place among Turkic plant names, generally connected with forest and mountain flora. Hungarian plant names were researched by Rácz [7], who considered dog's, wolf's, pig's names among the most common ones among those with an animal element. Komi phytonyms were discussed by Rakin [8]. Most of these works analyse many plant names in detail, but somewhat selectively, and without statistics.

Specific animals were discussed in numerous articles. For example, the Dutch ethnobotanist T. van Asseldonk examined the use of 'pig' in plant names in French, German, Dutch, and Flemish [9]; T. B. Haber - on dogs and other canines in British and American English [10]. Some authors tried to find explanations of naming in traditional culture [11]; such an approach is typical in ethnolinguistics. Still, most researchers limited themselves to comparing plants' organs with these or those body parts of animals, as well as pointing out some of their features - that is, their properties and characteristics. So, van Asseldonk mentioned the toxicity of some 'pig'-herbs and the fact that some of them are eaten by pigs [9]; Haber included worthless, inferior, harmful, and not cultivated plants to the list of 'dog' plants, adding those serving as dog's medicine, curing dog bites, and beneficial to dogs [10]. In a work based on Slavonic plant name data, V. Kolosova [12] discerned sixteen motivations (plant features which were the reason behind the naming) for animal names, a plant's shape, surface, colour, size, habitat, status, and a plant being animal's food or medicine among them. N. Vajs in her article [4] attempted to set up correspondences between the appearance of plants and an animal body part forming the basis of nomination (Fig. 1).

More specifically plant names with a bear component were discussed in articles by Brodskij [13] on FinnoPermic and Balto-Fennic languages and by Kolosova [14] on Russian dialects. We should also mention Dahlstedt [15] who in more depth discussed many bear berry names in northern Scandinavia. He notes that bear- in names of berries in northern Scandinavia is probably pejorative, because it is often used to berries regarded as inedible. However, it can also refer to the colour, because unripe berries can be red or maroon.

There was often no direct relationship between the actual habitat of an animal species and the use of animal metaphors in local plant names. There were for instance names with 'lion', 'monkey' and 'dragon' in the Nordic languages, and 'elephant' and 'lion' in various Turkic plant names $[6,16,17]$. The Tudor naturalist Turner recorded bearefote (nowadays still called bear's foot) in English already in 1538 for Helleborus [18]. No wild bears were present in the British Isles at that time. They were probably extinct already by $500 \mathrm{AD}$ or somewhat later [19].
Bear-related plant names can be considered semantically transparent or in some cases semi-transparent in Berlin's terms [20], yet we are talking about once traditional, but now highly literate societies. Therefore understanding the motivations of plant names is not easy. Plant names and the naming of plants subsume a great many aspects: synchronic, diachronic, structural, semantic/associative, systemic/variational, etymological, geographical, social, etc. There is also a question of the emotive/situational value of plant names and the handling of plant names in literary contexts. Many plant names reflected folklore motifs and mythological ideas. In addition, there were normative aspects in terms of diffusion and normalization of plant names. It is therefore not only a question of the supposed origin and motif of denomination (why was a plant X called Y?). Some names were for instance just translations of the Latin name, and especially the pre-Linnaean Latin names must be considered, cf. Turner's bearefote [21].

Large charismatic animals have always attracted people's interest, and therefore there exists a rich cultural history associated with such animals. Predators especially have exerted a special attraction in art, folklore, myths, rituals, and other cultural expressions. This applies in particular to the bear (along with wolf) that has played a central role in people's perceptions across Eurasia. Bear worship has for instance been common all over the circumpolar area [22-26]. Some peoples even regarded bears as their ancestors $[27,28]$. Vice versa, in Slavonic etiological legends bear is a human transformed into a beast as a punishment for some sin. South Slavs celebrated special "bear days" [29].

Bears are found all over the northern hemisphere [30]. Very much was written about the bear in folk perception and as a prey for hunters. Our relationship with bears seems eternal, ranging from prehistoric human relation to the cave bear right up to the bear's place in today's popular culture $[31,32]$. Several ancient (Pliny the Elder, Claudius Aelianus), medieval (Hildegard of Bingen, Albertus Magnus, Bartholomew of England), and Renaissance authors (Olaus Magnus, Conrad Gessner) dealt with the bear in detail. The scholarly literature on the bear in human history is extensive and covers many aspects [27, 30, 33-36].

Echoes of ancient ideas on some "equality" of humans and bears have been alive in ritual practices until recently. For instance, in Älvdalen, in the Swedish province Dalecarlia, a fiancée was called 'she-bear', and there are many expressions connected with the rituals before a wedding when the bear metaphor is used. Björn-grånka is the local term for a spruce tree, Picea abies (L.) H. Karst, which was set upright against the door of a farm where someone had the banns of marriage published. This was done to prevent 'the bear' from coming 'out of hibernation' [37]. In Russian wedding songs the bride is called медведииа 'she-bear' [38]. 


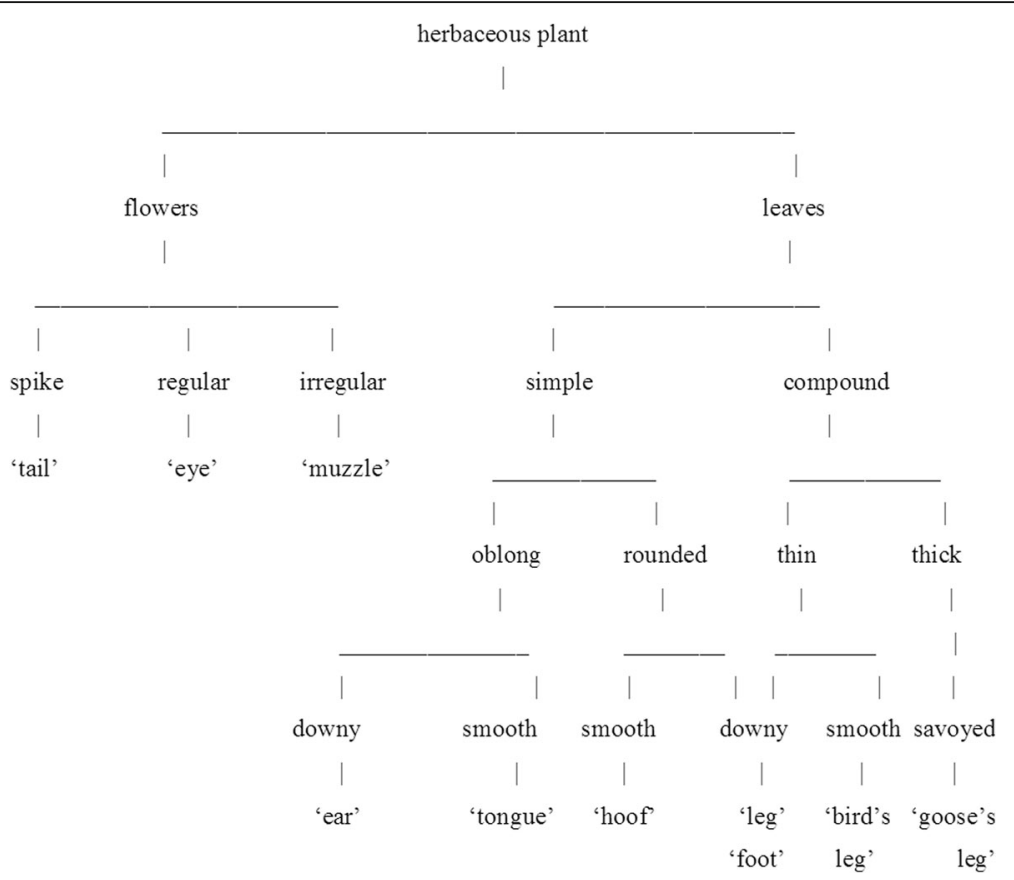

Fig. 1 Correspondences between the appearance of plants and animal body parts based on nomination (adapted from [4])

As a charismatic animal, hunted, revered, and feared, the bear also has many euphemisms in Eurasia [29, 39-41]. Strictly speaking, bear/björn/bjørn/Bär in the Germanic languages literally mean "the brown one", is an old euphemism [41]; Russian literary and common name for bear is also a euphemism literally meaning "honey eater"; all of them are used instead of old Indo-European name "rkso-

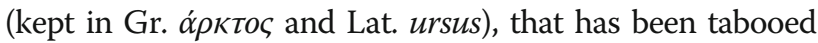
for many centuries [42]. The Hungarian language uses a Slavonic borrowing medve for the same reason.

The objectives of the research were to answer the following questions:

a. which plants were associated with the bear in folk plant nomenclatures?

b. why certain plants were considered 'bear plants' (i.e. eventual links to local perceptions)?

c. is there a common perception of the same taxon over larger areas?

d. what are differences and similarities of the patterns among different languages?

e. do plant names with 'bear' component always link to the animal 'bear'?

f. which word-formative models are used for creating 'bear'-names?

\section{Methods and sources}

Within this work we concentrated on plant names related to the Brown Bear (Ursus arctos Linnaeus, 1758) historically or still today co-habitating in the areas of the distribution of selected language groups (Fig. 2). We give an overview of using the bear as a prefix in Eurasian folk plant names (sometimes it is not a prefix but just a word meaning 'bear' or 'little bear' used as a metaphorical name). The names belonging to the official nomenclature were not analysed, unless they coincided with the folk ones.

Only species names were considered. The cases of bearnomination for individual natural objects, as, i.e., It. faggio dell'orso 'bear's beech' for one famous big tree at Mt. Tranquillo, near Pescasseroli village, are not analysed.

Plant names containing the root bear-were analysed in the spoken dialects of several Eurasian languages: Slavonic (Belarusian, Bulgarian, Croatian, Czech, Macedonian, Polish, Russian, Ruthenian, Serbian, Slovak, Slovenian, Sorbian, and Ukrainian), Germanic (English, German, Danish, Norwegian and Swedish), Romance (French, Italian, Latin, Sardinian, Friulian), Finno-Ugrian (Estonian, Finnish, Hungarian, Izhorian, Livonian, Saami, and Votic), Turkic languages (Bashkir, Chuvash, Kazakh, Kirghiz, Tatar, Turkish, Uyghur, and Uzbek) and other languages (Albanian, Lithuanian). The authors also considered the cases when the plant was unidentified, but the citation from respondent explained the using of a bear-name. The inner form (literal translation) of local names is given in square brackets.

The published dictionaries, ethnolinguistic literature, folkloric and ethnographic references based on primary literature and original field investigations were considered. 


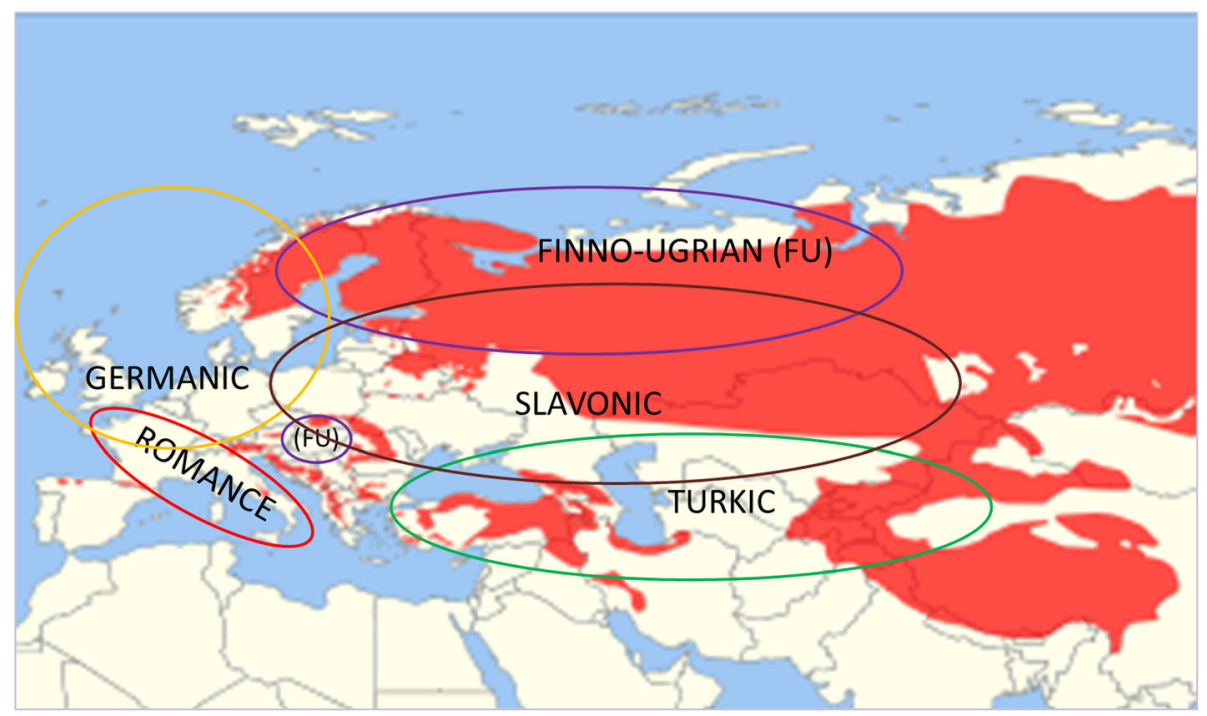

Fig. 2 Language groups reviewed in this publication and present geographic distribution of Brown Bear (Ursus arctos). Red - distribution of Brown Bear. Circles indicate the regions inhabited by the speakers of the language group very roughly. Base map (species distribution map) is created by the International Union for Conservation of Nature and distributed by Wikimedia according to Attribution-Share Alike Creative Commons License (https://commons.wikimedia.org/wiki/File:Ursus_arctos_range_map.svg)

Field materials from Abruzzo [Cianfaglione], Transylvania [Frendl and Papp], Estonia [Kalle and Sõukand], Transylvania [Molnár, Babai], and the Alps [Pieroni] are published for the first time. Data was extracted from the sources and entered in an Excel spreadsheet. Nomenclature followed The Plant List database [43] for plants and Index Fungorum [44] for fungi. The dataset supporting the conclusions of this article was included within the article as its (Table 1). The dataset has the following structure: valid nomenclature taxon, original source taxon identification, local plant name and its literal translation into English, language and geographical area of fixation of the plant name, motivation, and the source of the information. The column containing motivations was formed on the basis of the material extracted from the sources.

\section{Are all bears in plant names really bears?}

There is a particular problem in the interpretation of the bear plant names. The bramble (Rubus subg. Rubus) was called björnbär ["bear berry"] in Swedish. This plant name was recorded already in 1643 in Sweden. But the reasoning behind this name is not easily clarified. There is nothing bear-like about it, and bears never appear in the area where it grows along the southern Swedish coasts. One possible explanation is that another folk name is brumbär, and the first part brum has maybe been associated with the animal's name (although brum in this case actually means 'leaf', cf. English bramble, German Brombeere, etc.). Although most contemporary people connect björnbär with bears, it is not etymologically derived from historical roots connected to the animal bear [17]. On the other hand, the connections of Rubus spp. with bears in Belorussian, Ukrainian, Czech, Old-Polish, German, Italian, Estonian, and Votic cannot be explained by folk etymology.

Another example is the English name bearbind for Clematis vitalba L., Convolvulus arvensis L., and Polygonum convolvulus L., where the 'bear' element historically comes from Old English beow 'barley' rather than the Old English bera 'bear', and thus the whole name literally means the plant that binds barley $[45,46]$. Similarly, it is bere (also 'barley') rather than bera, that lies behind bear-barley, a Northumberland name for Hordeum hexastichon or tetrastichon, which thus has nothing to do with bears, but literally means "barley-barley" [47].

Thiselton-Dyer [1] used bear's-wort as an example, which, according to him, "is rather to be derived from its use in uterine complaints than from the animal". The explanation makes a lot of sense as Eng. bear has (at least) two meanings, including 'the animal bear' and 'to give birth', which lent good material to folk etymologists. If there was an herb that helped giving birth or was good for gynecological disorders, it could get the name of bear's-wort. The situation was the same with Germ. Bär 'the animal bear' and gebären 'to give birth'.

Words from which the later words were derived are a critical issue also in other quasi bear plant names. The main similarities between Est. karu 'bear' and the adjective karune 'hairy' made contemporary speakers take them as being cognates. The words for 'bear' (North Estonian and standard Estonian karu, South Estonian 


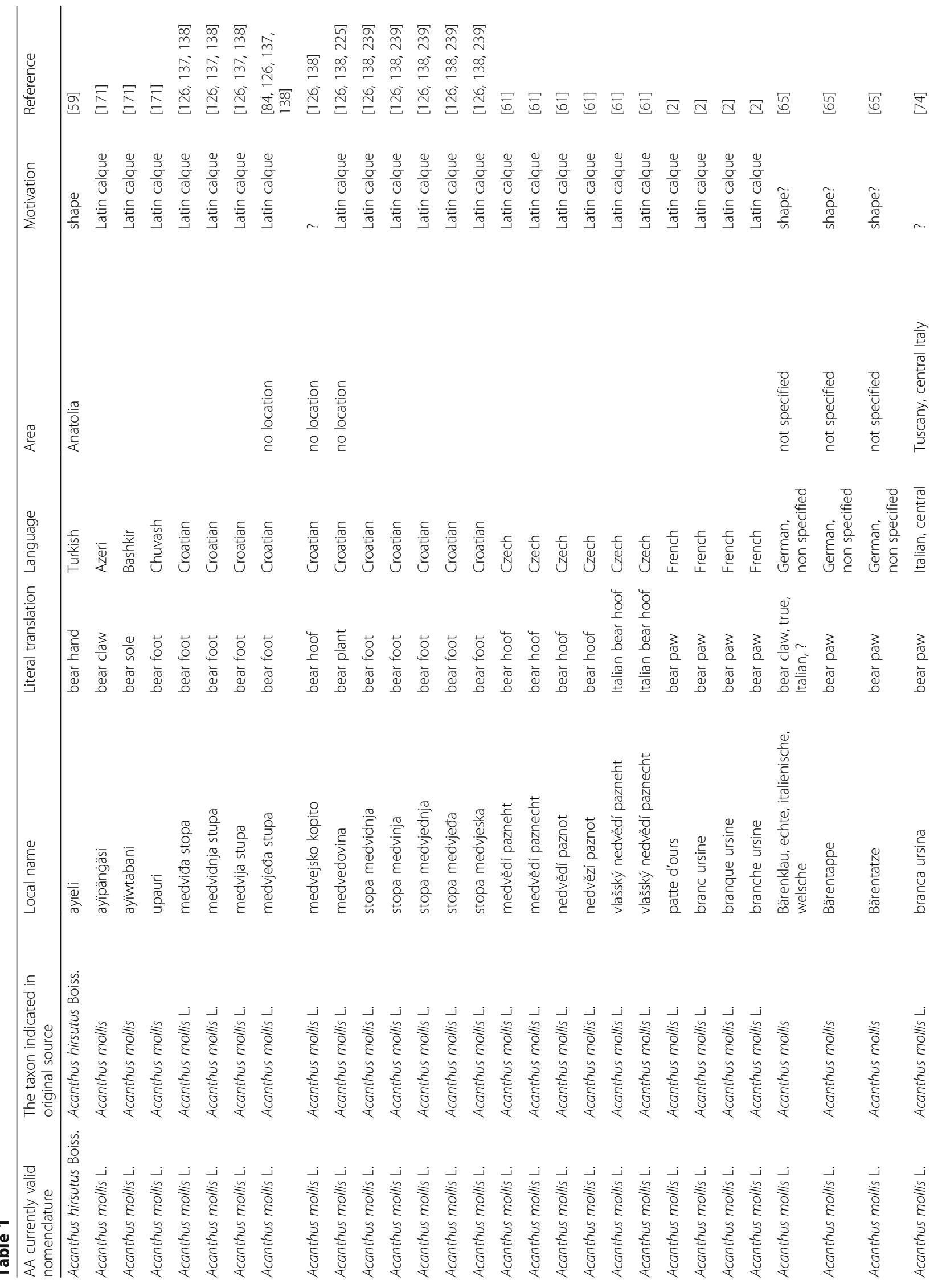




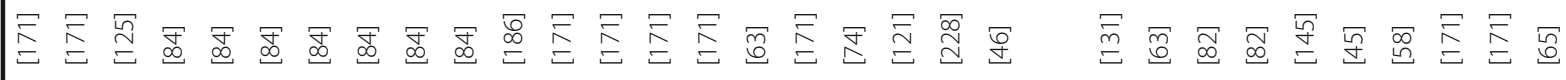

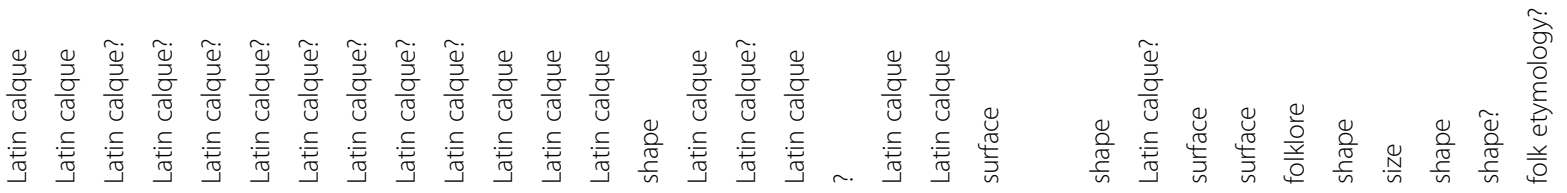

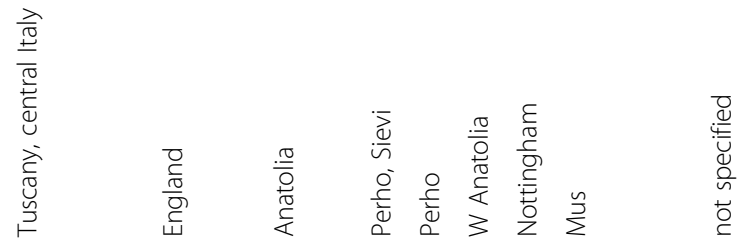

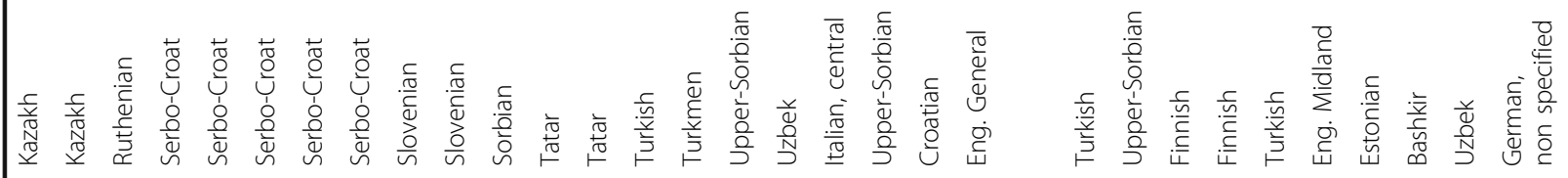

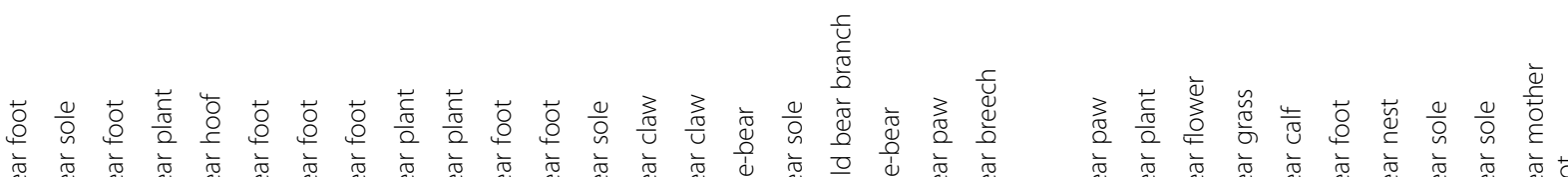

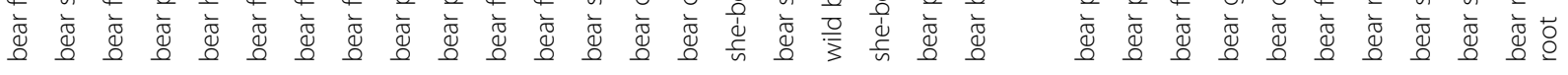

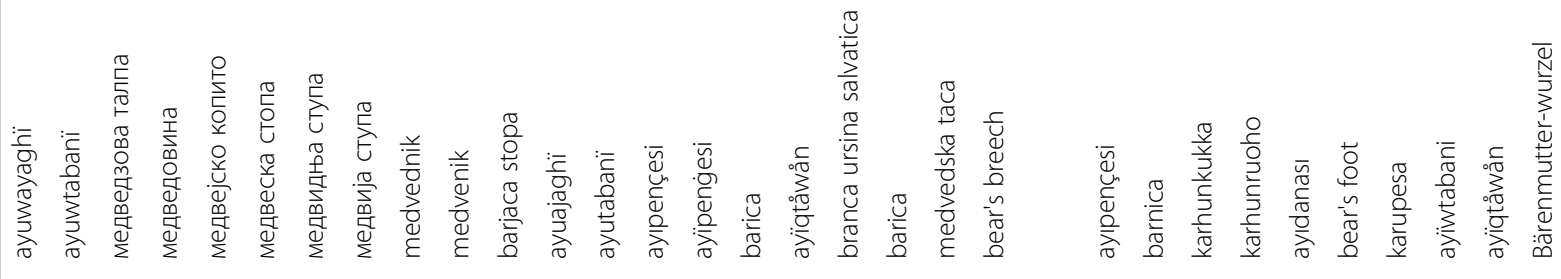

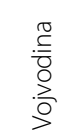
(a) 


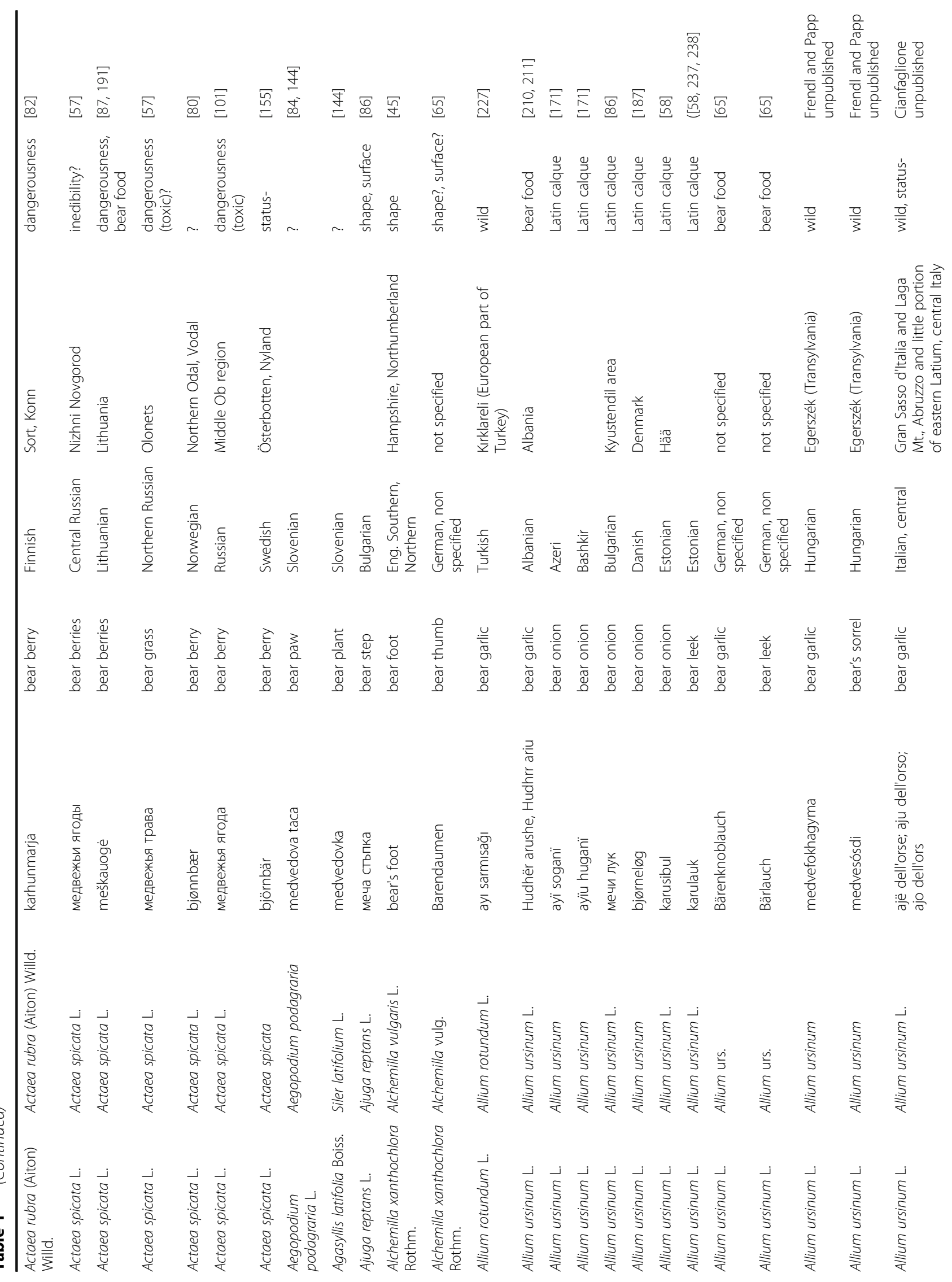




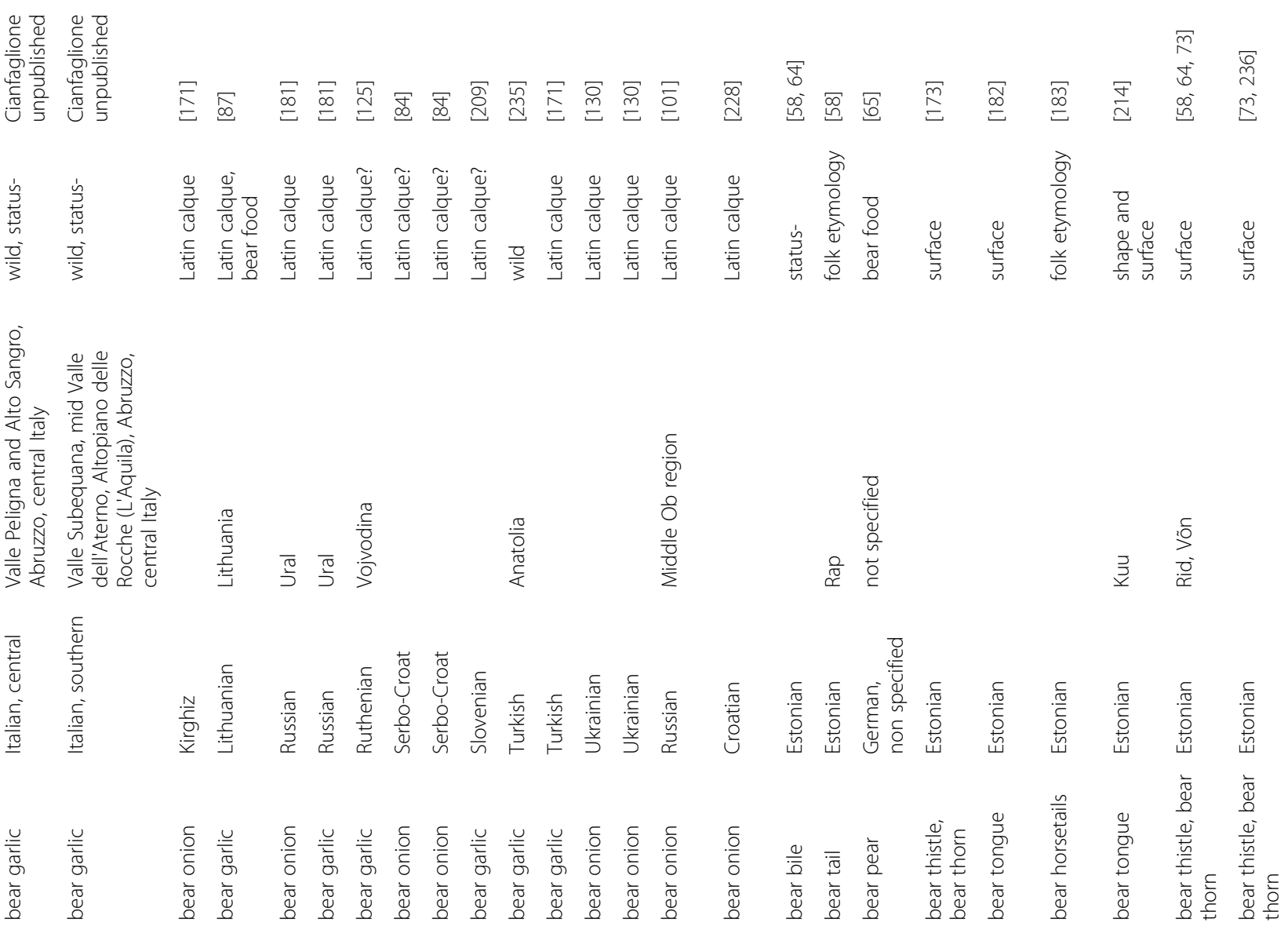

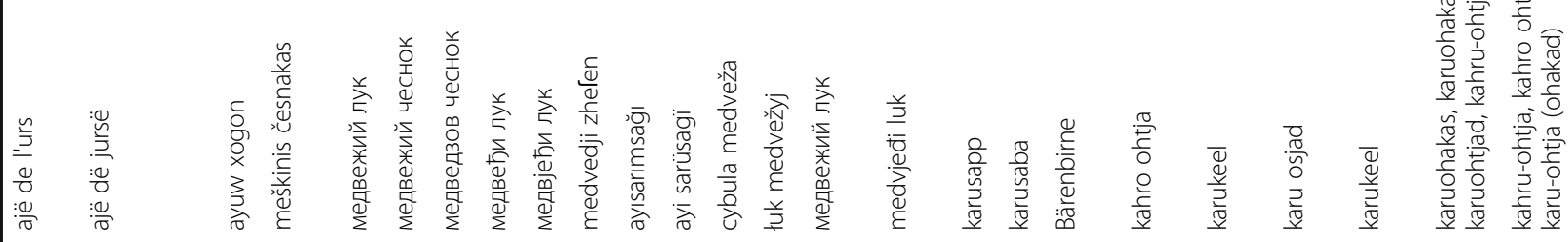

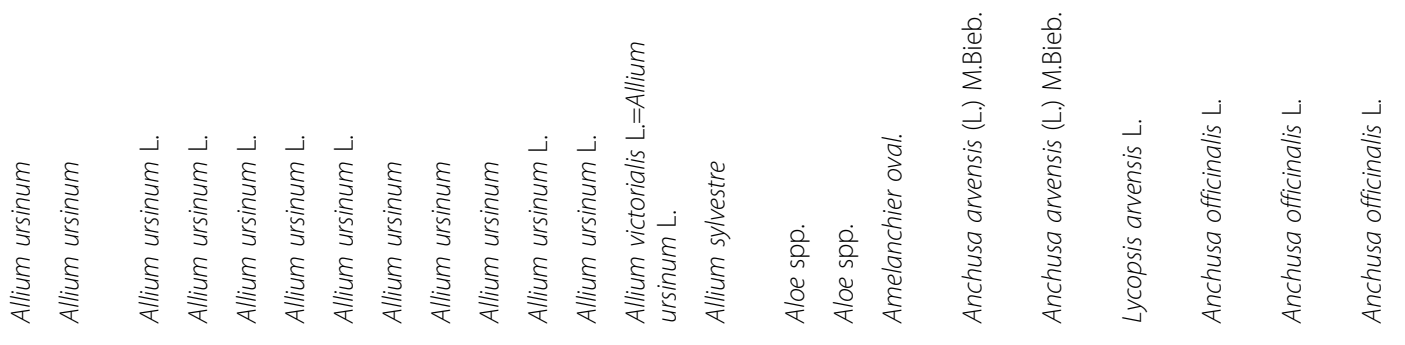




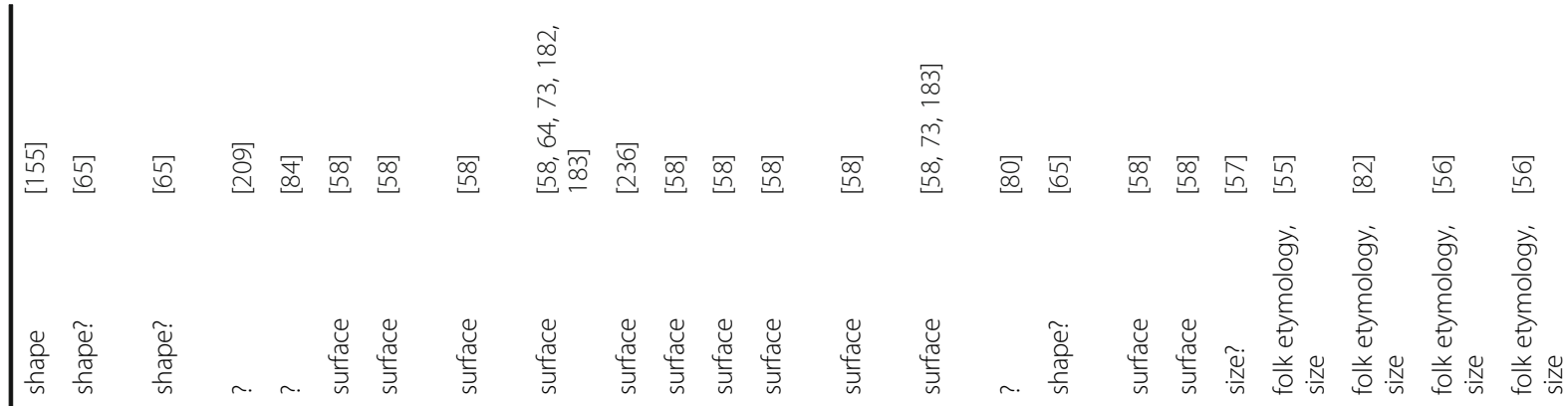

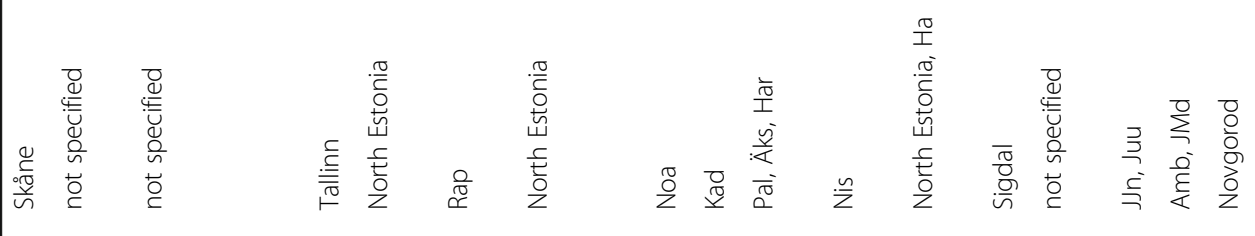

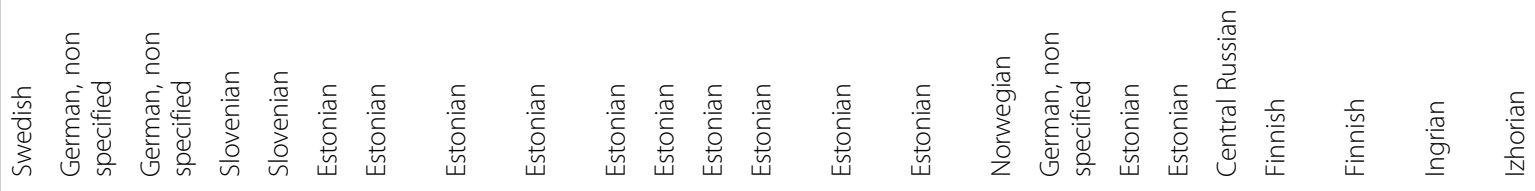

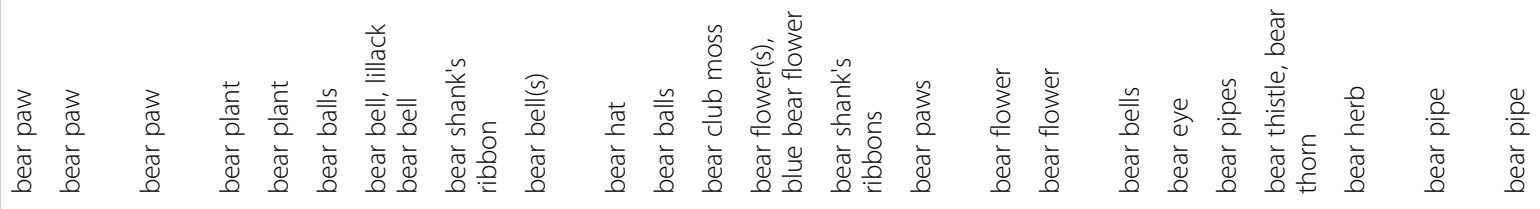

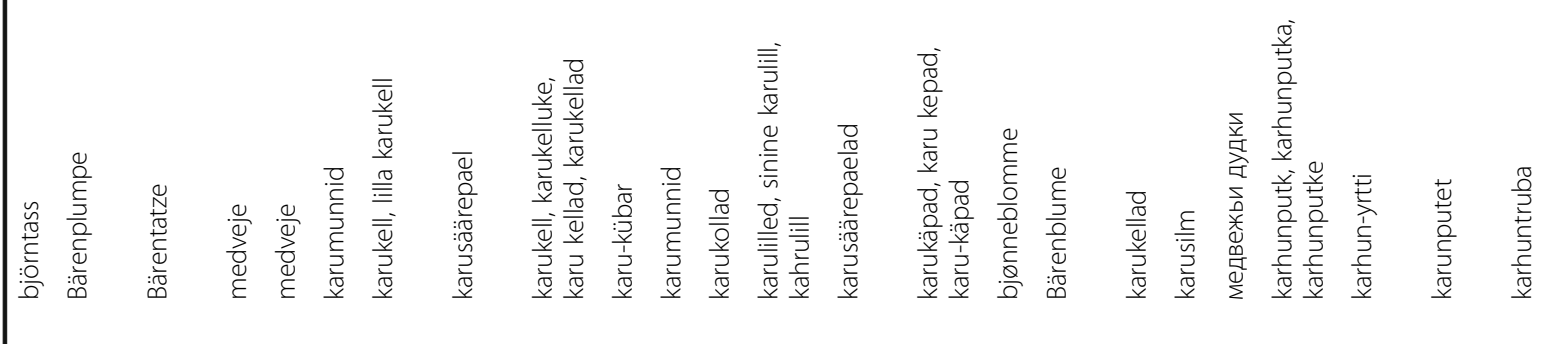




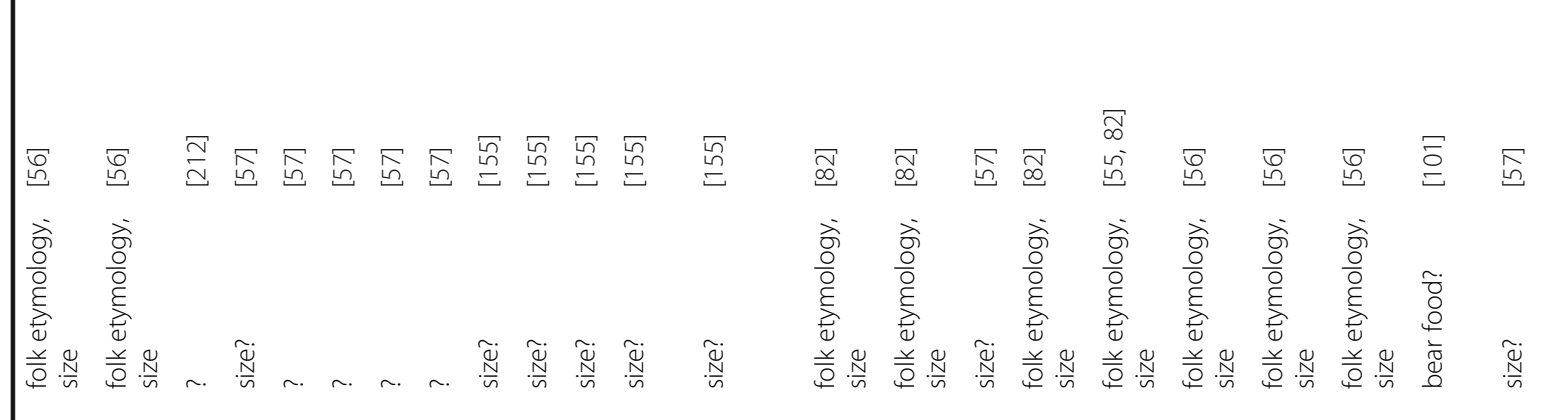

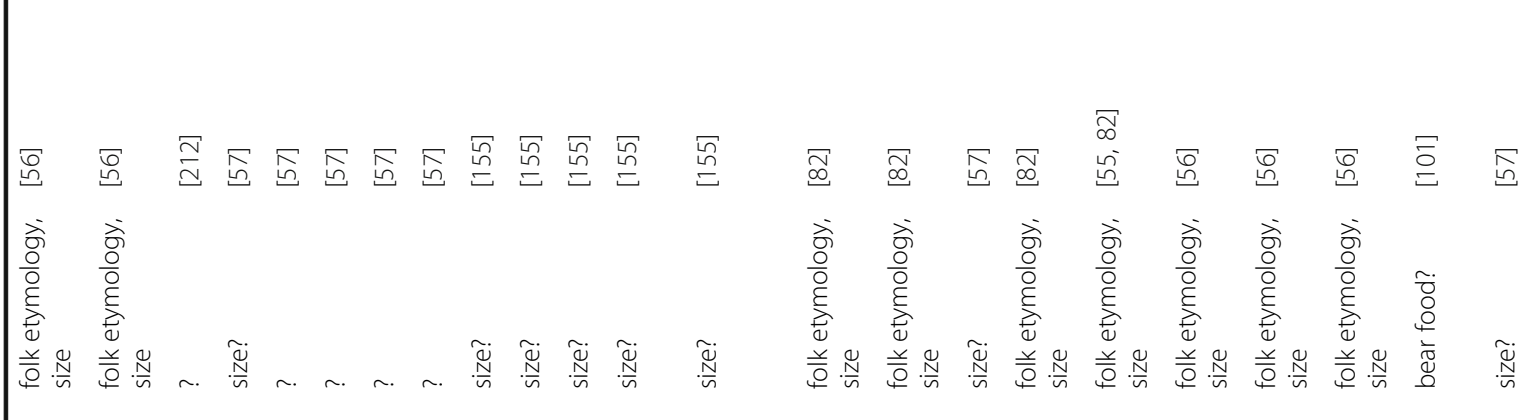

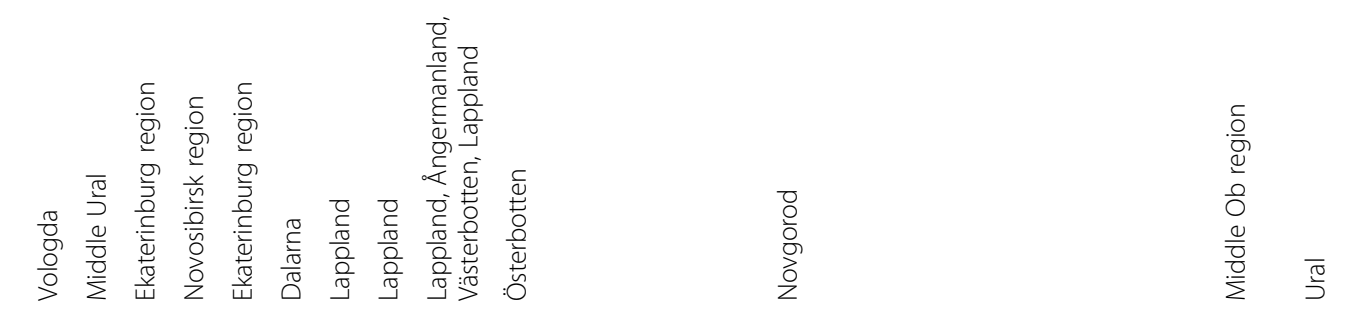

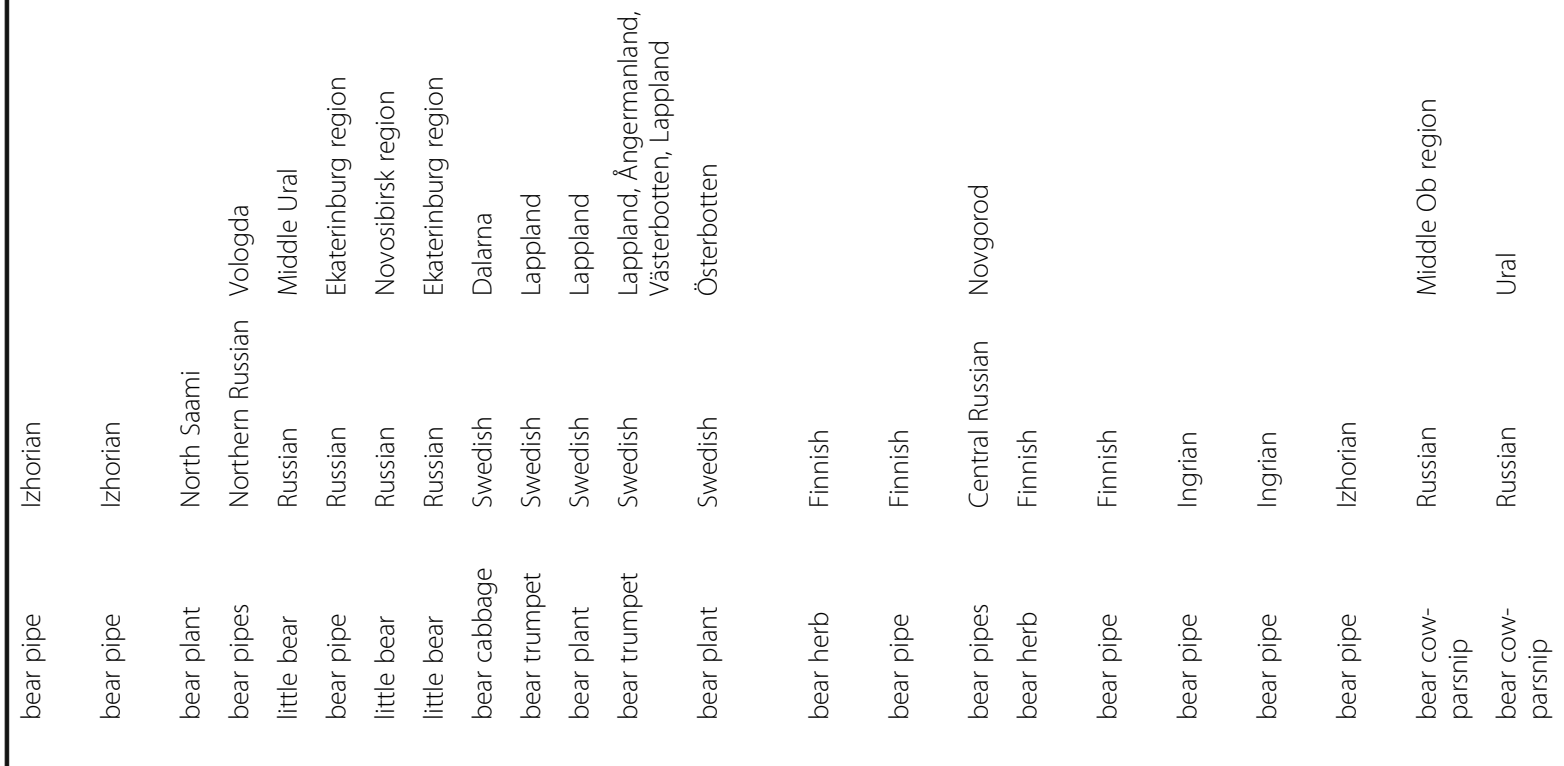

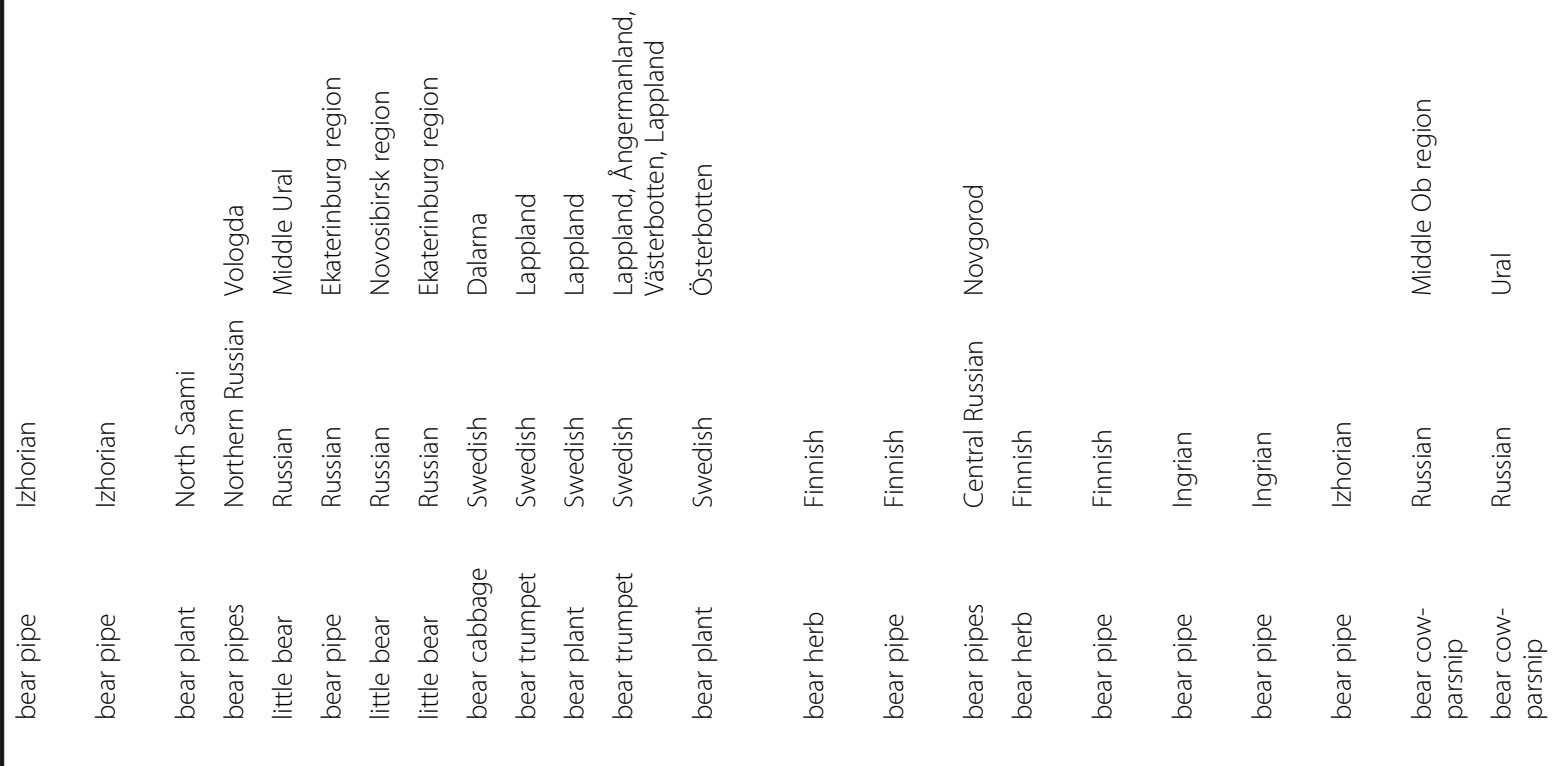

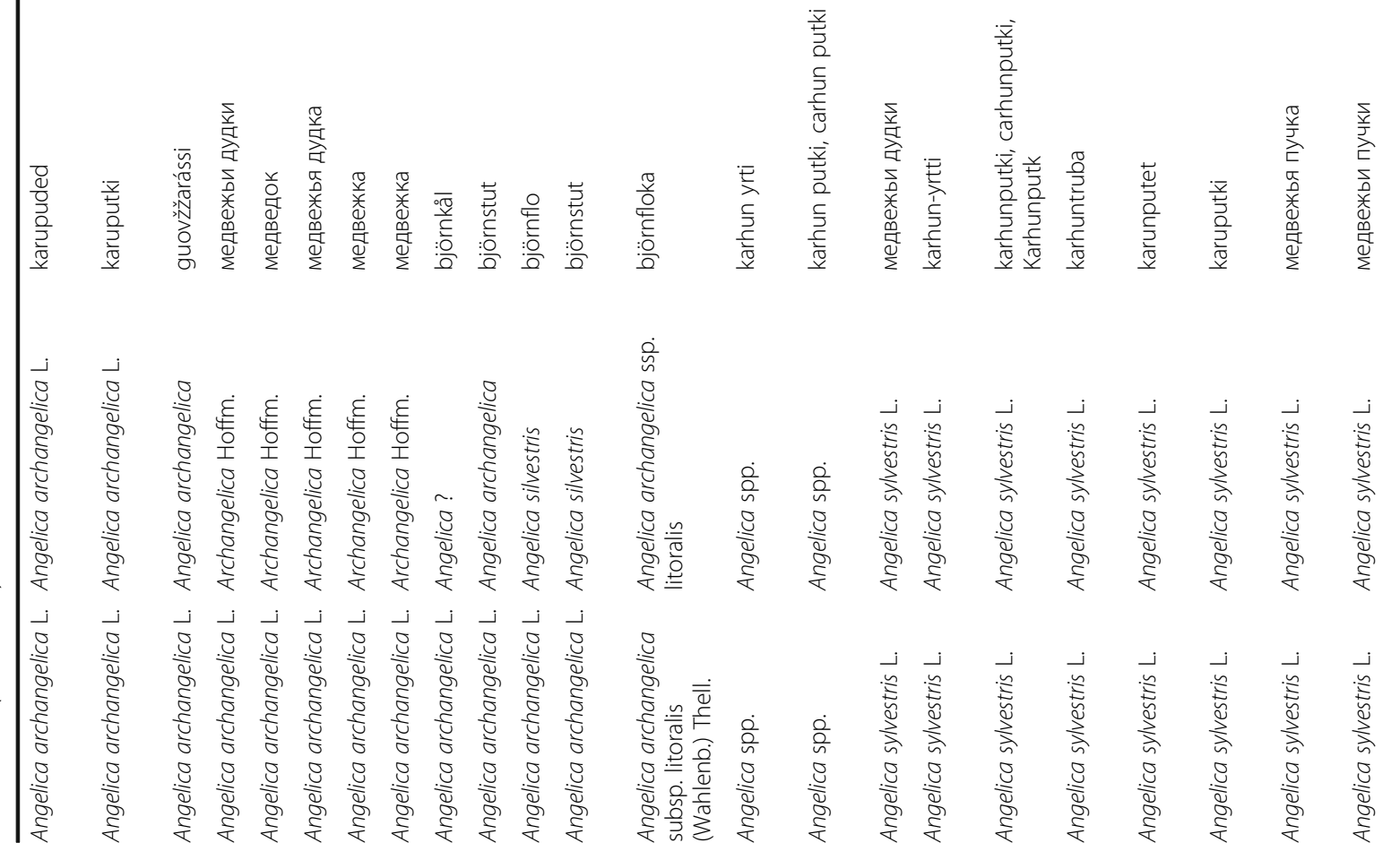

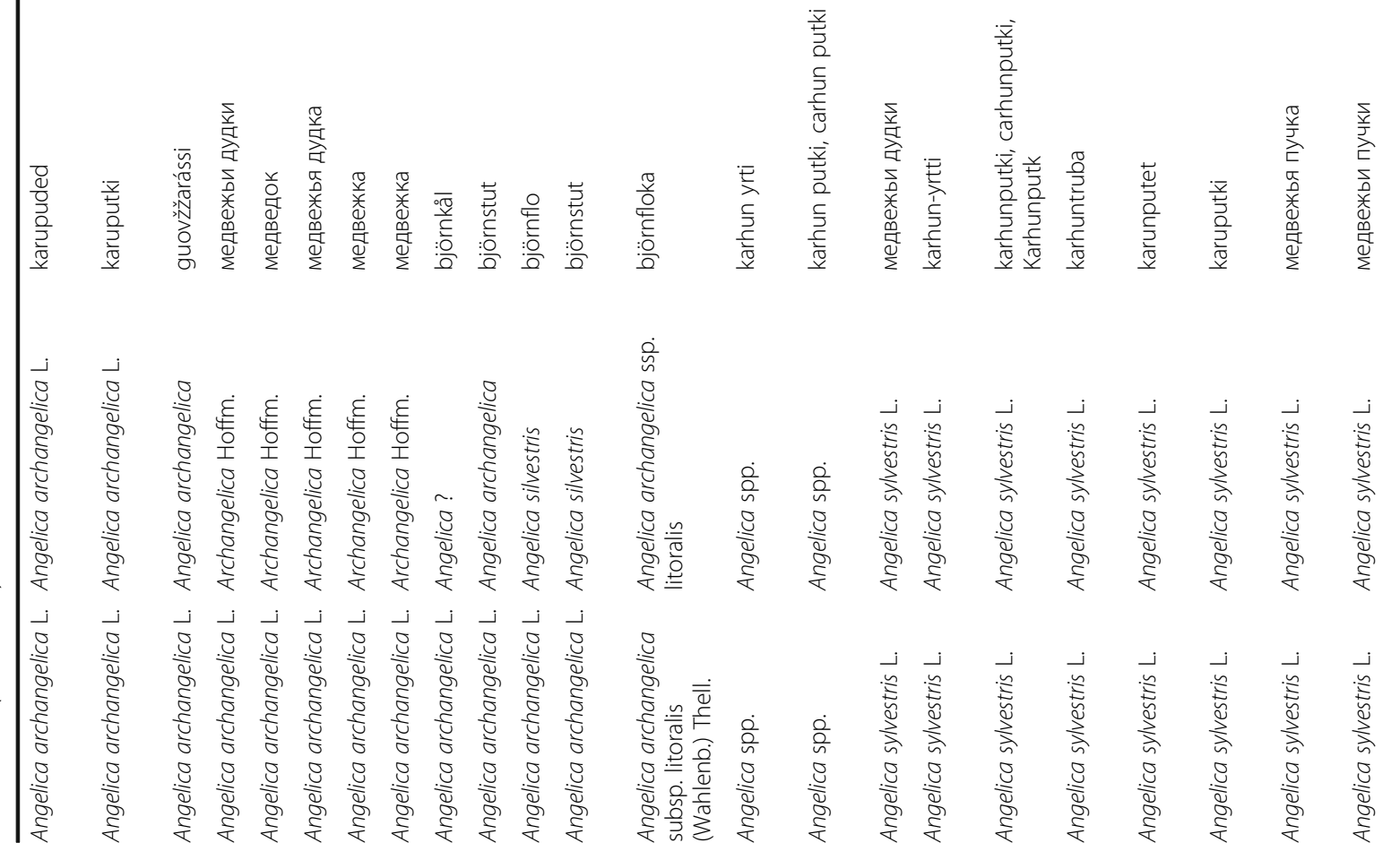




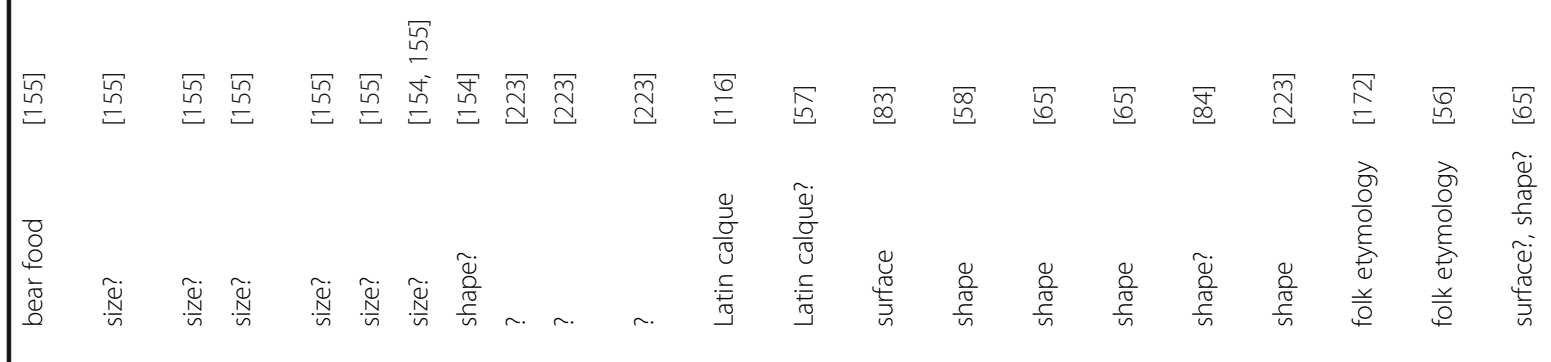

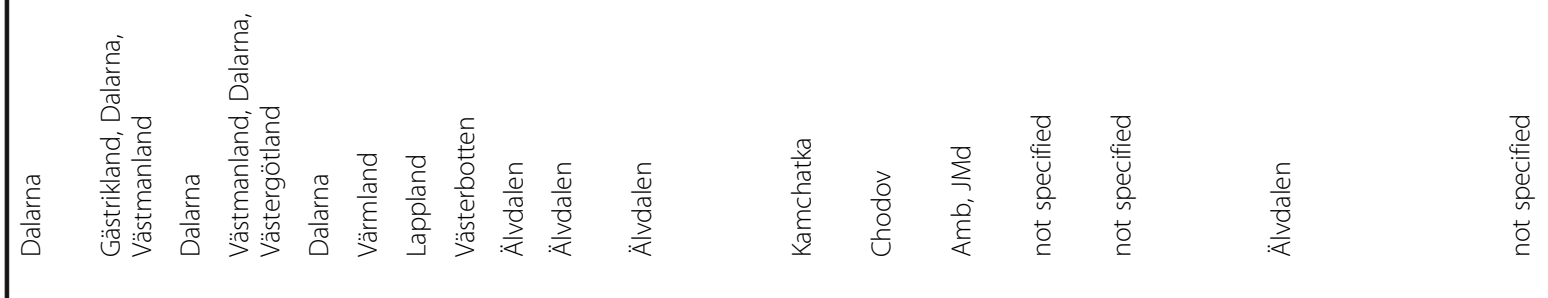

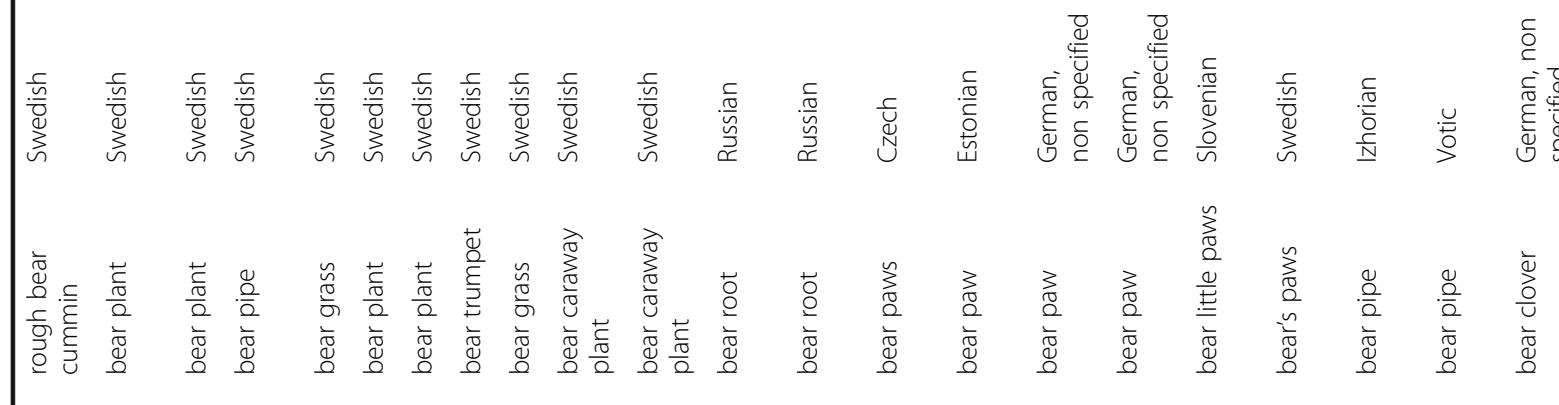

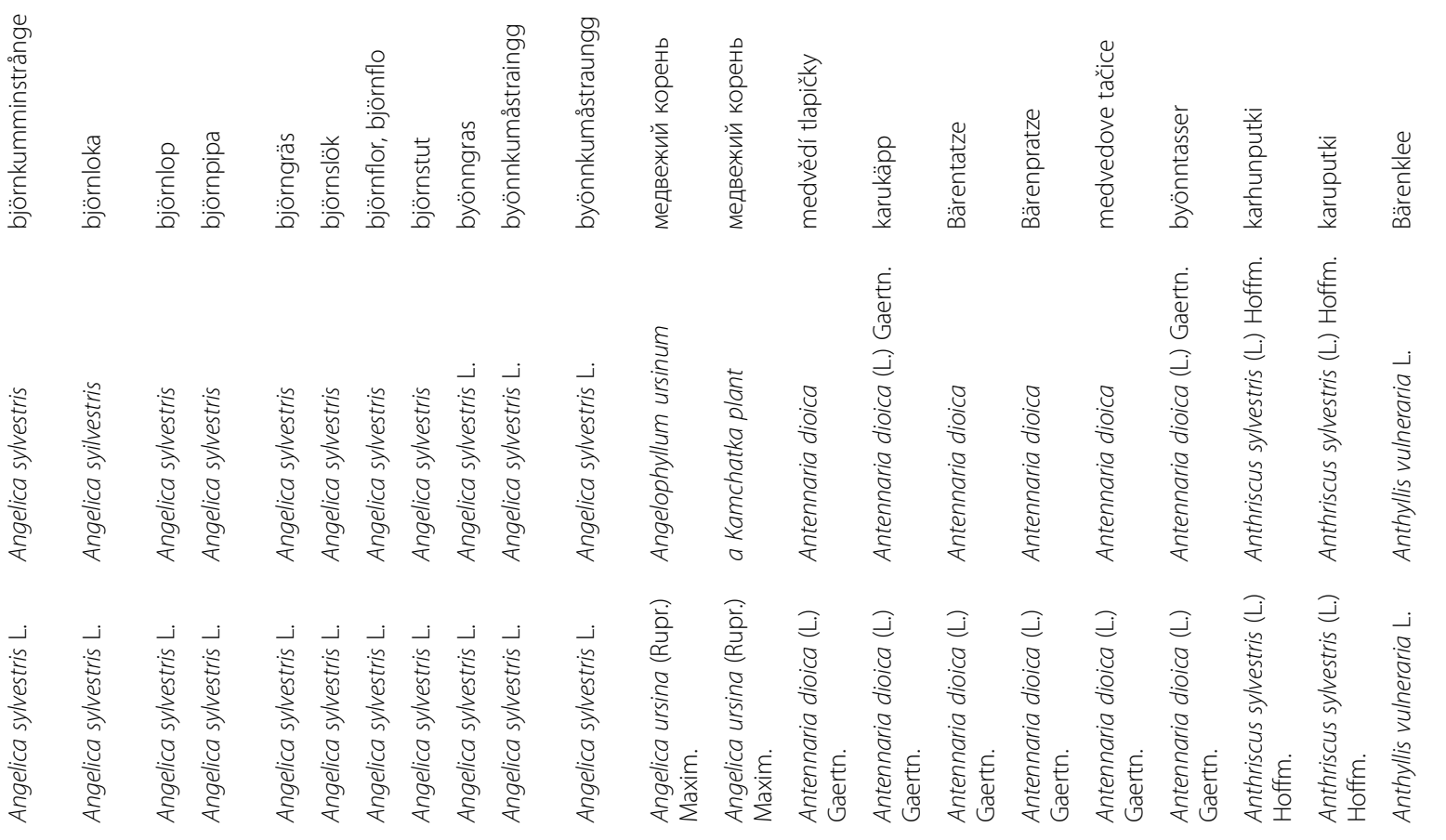




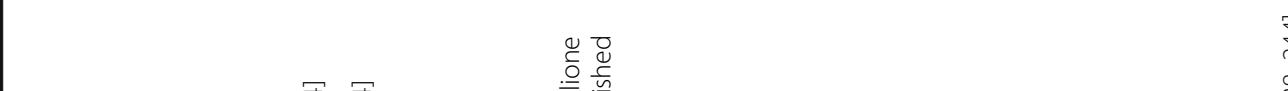

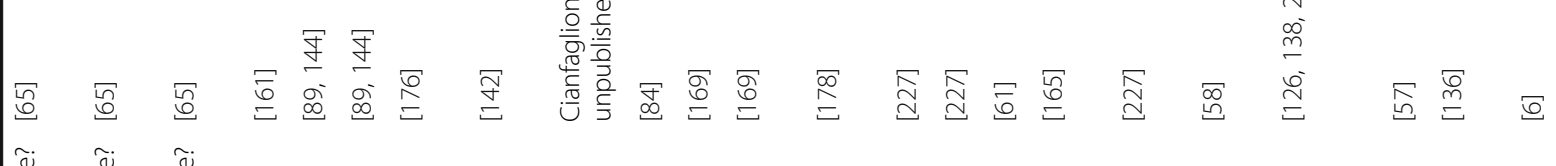

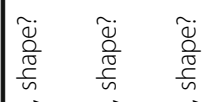

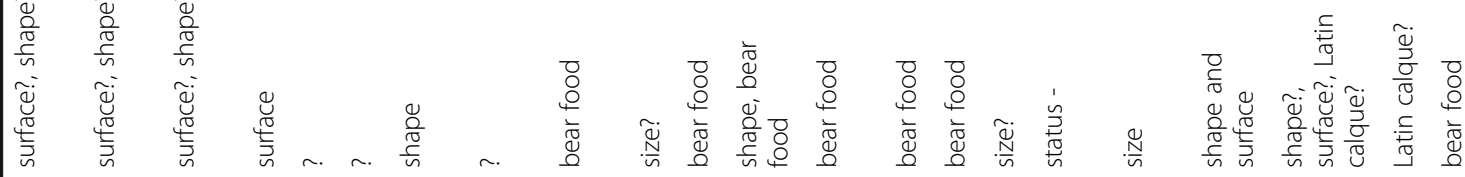

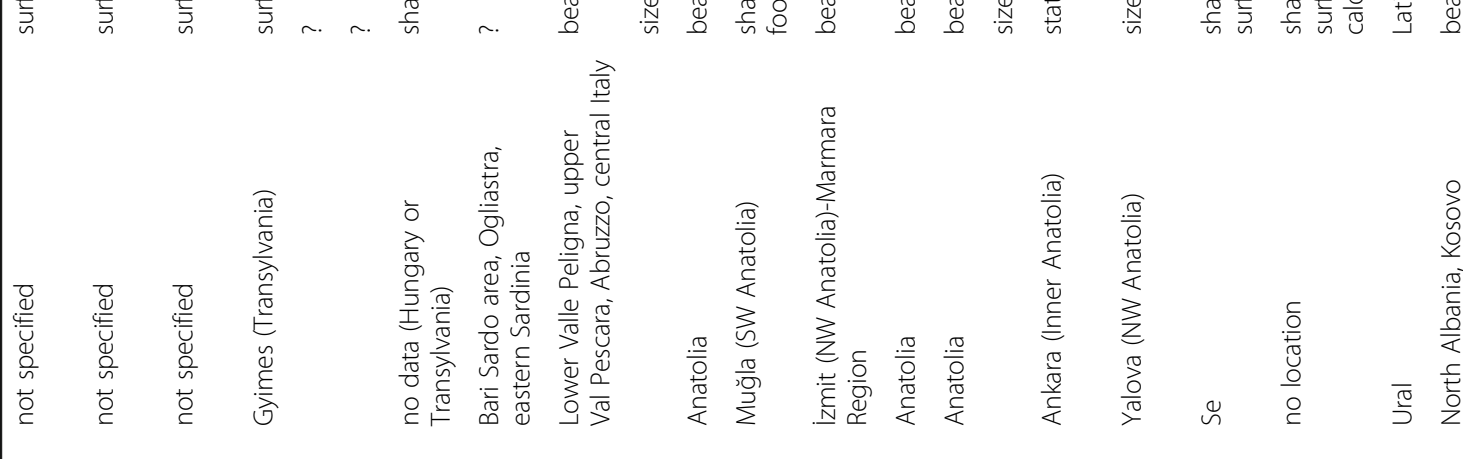

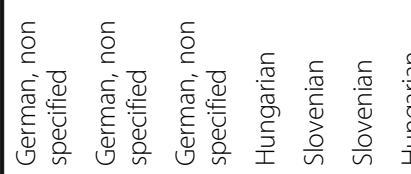

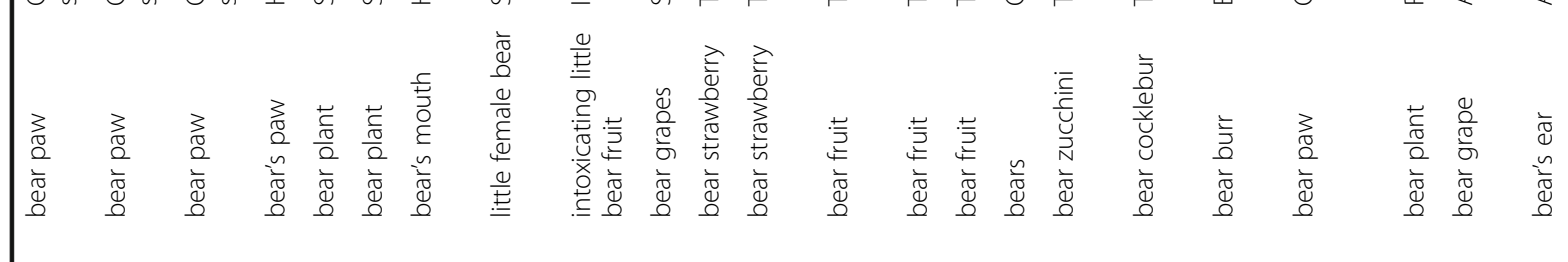

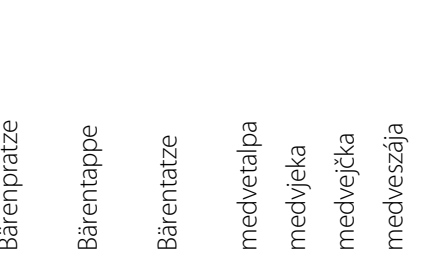

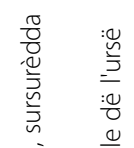

$\stackrel{+\pi}{\stackrel{0}{0}}$

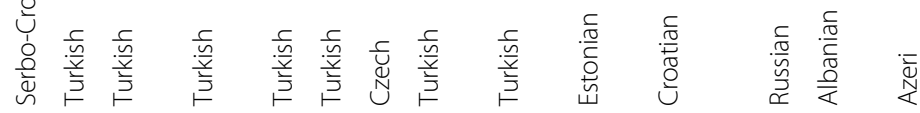

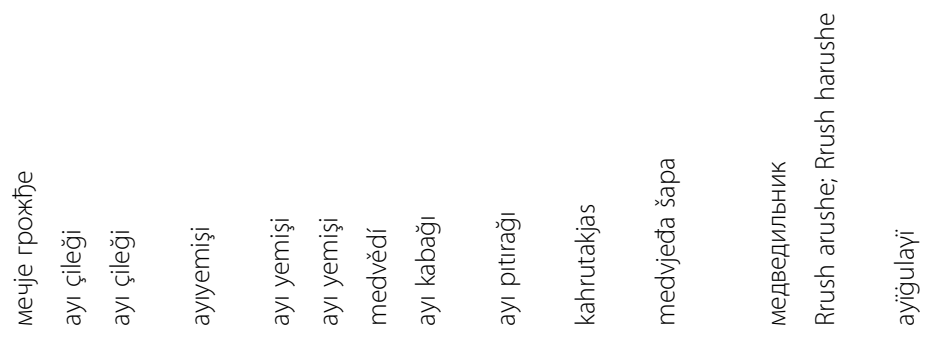

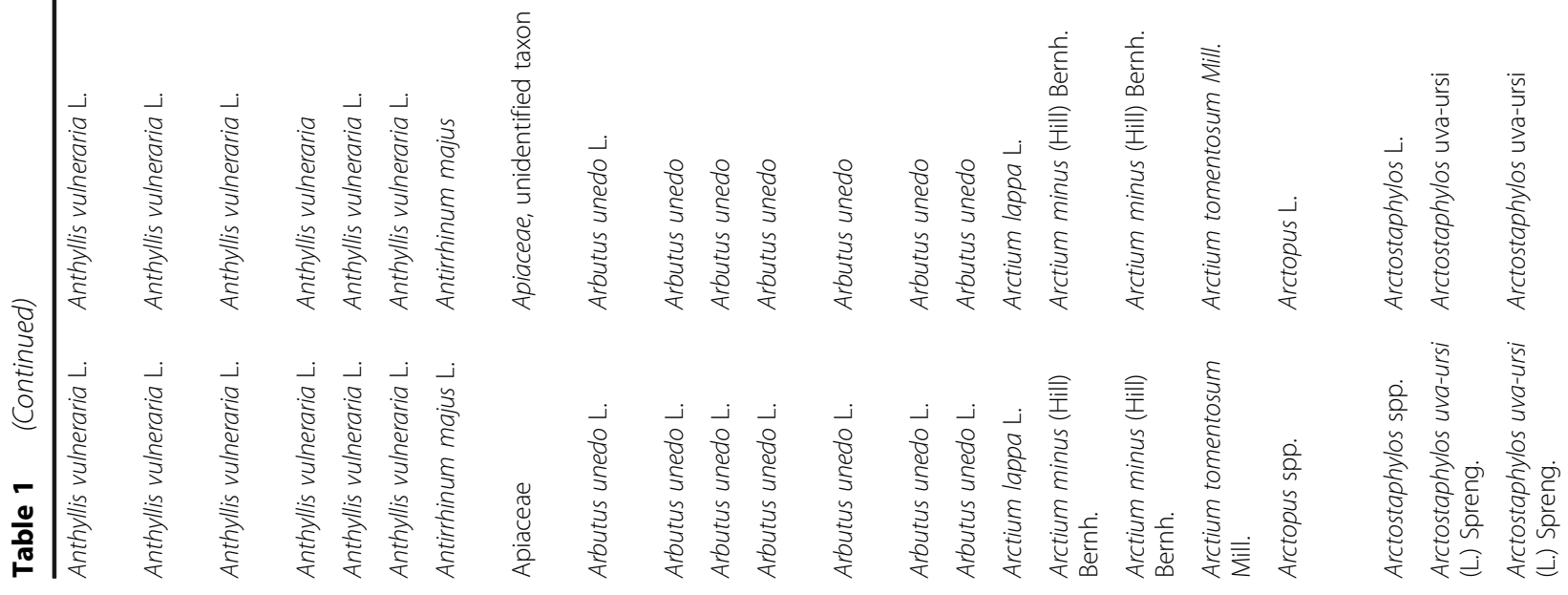




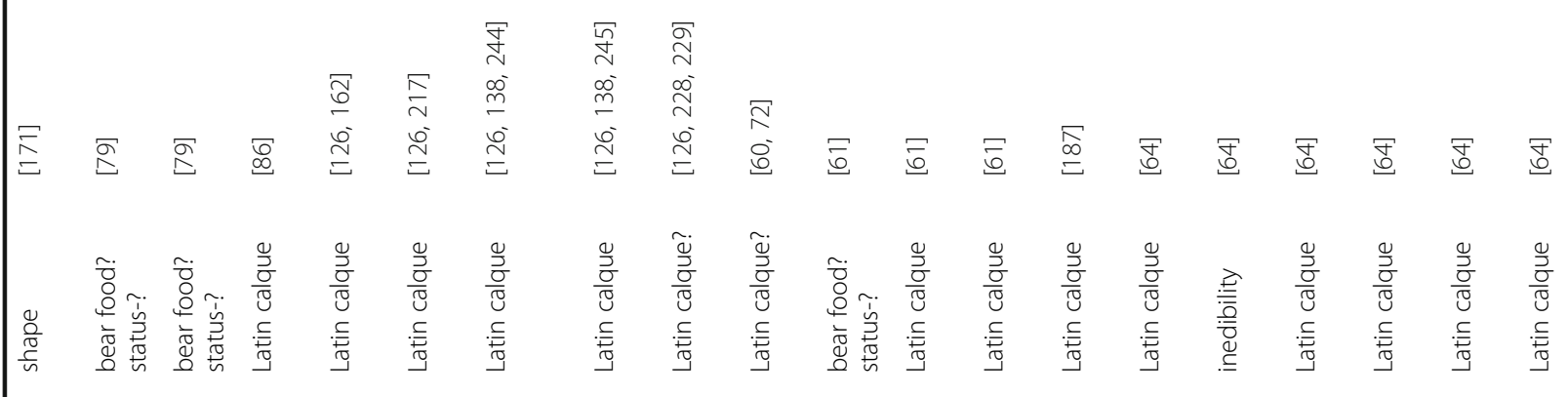

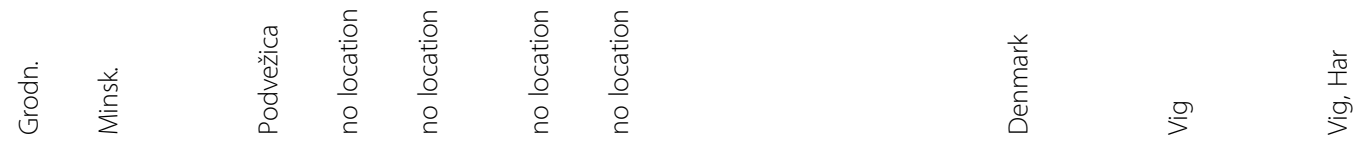

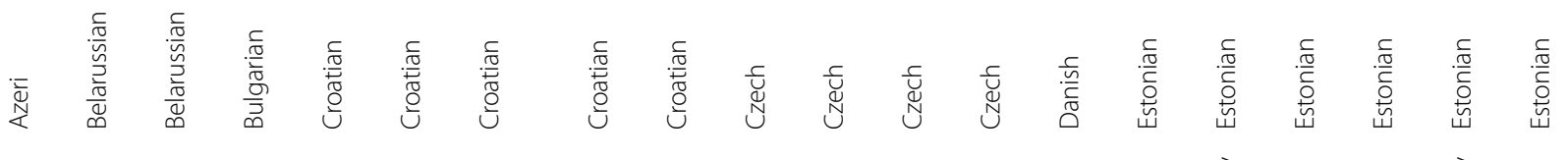

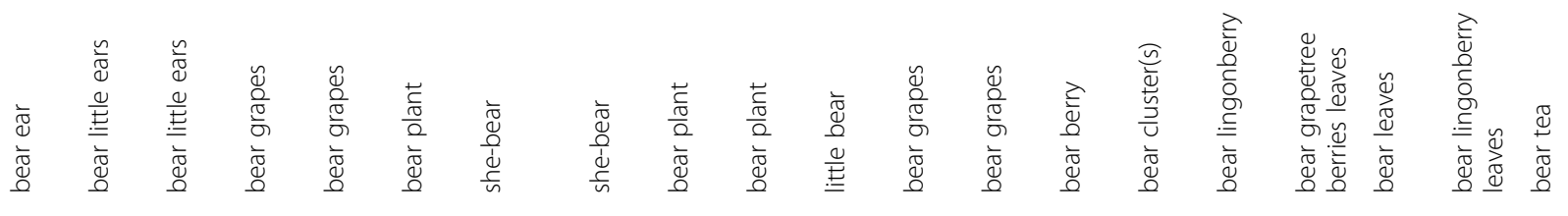

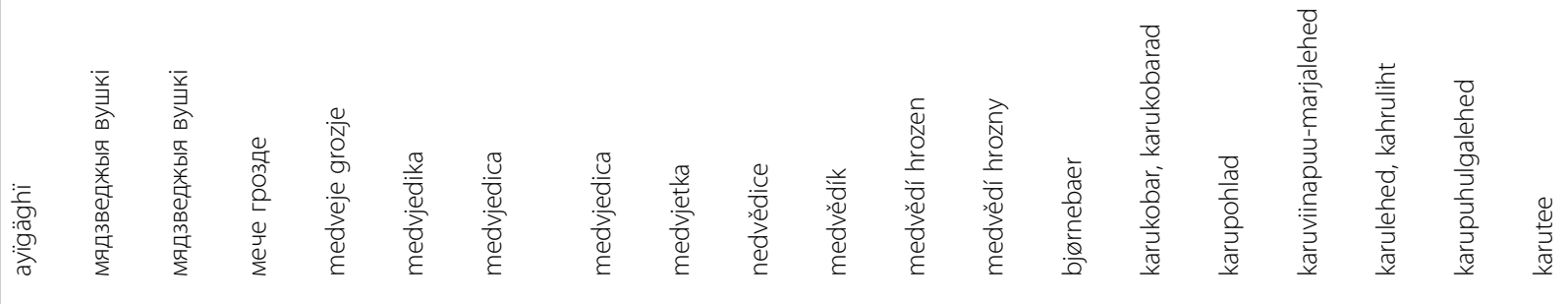

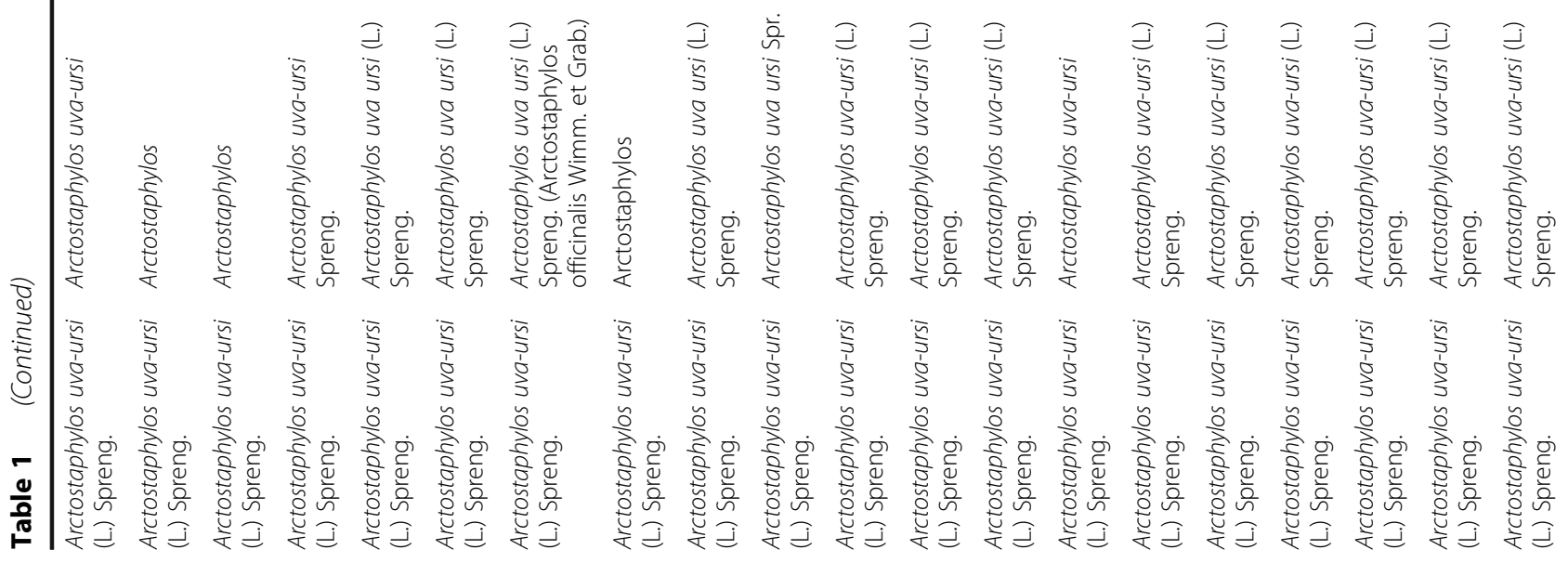




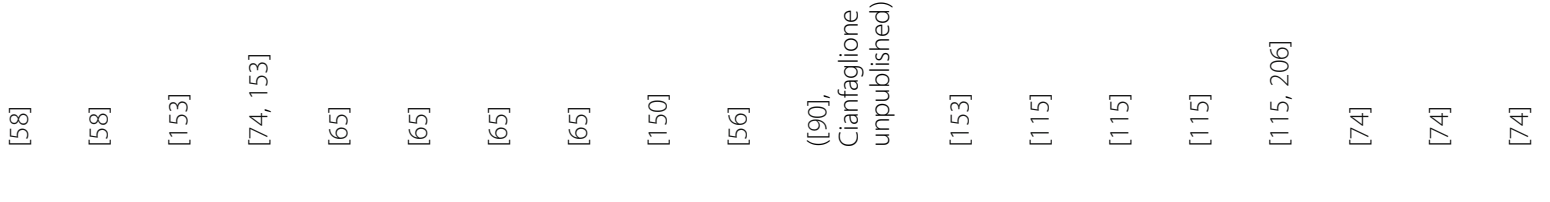

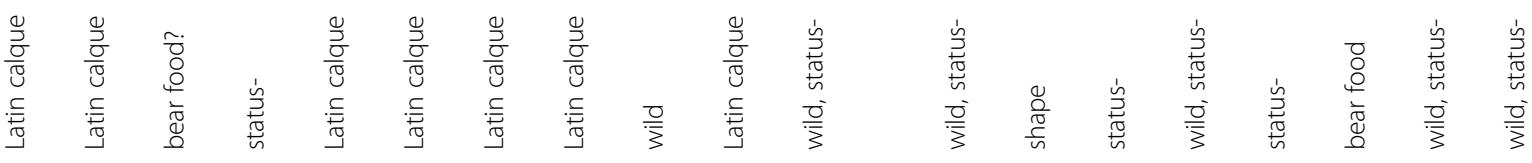

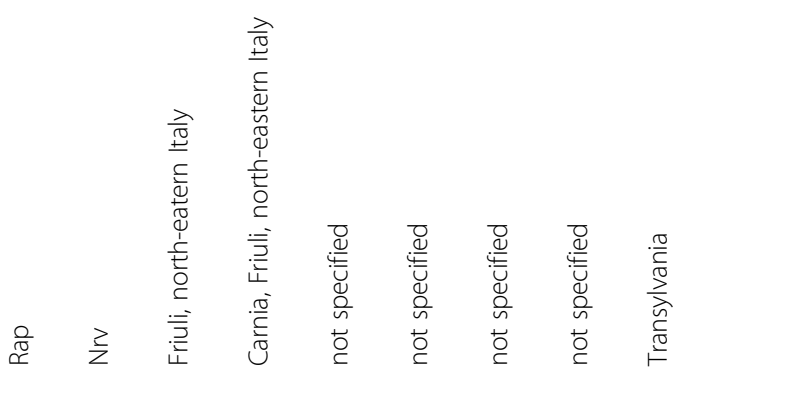

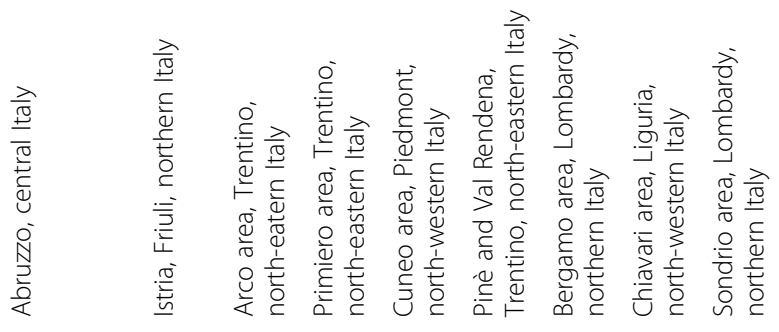

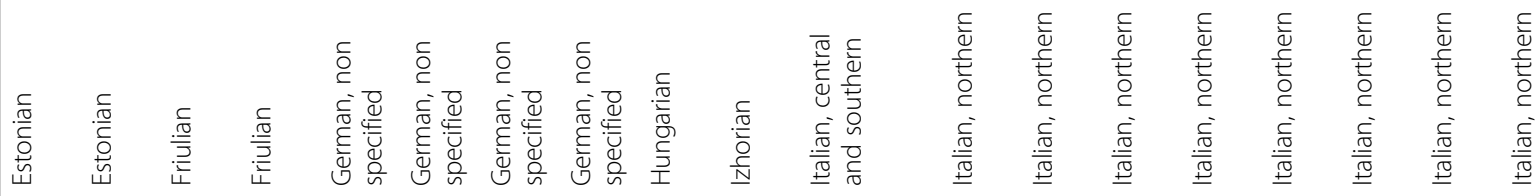

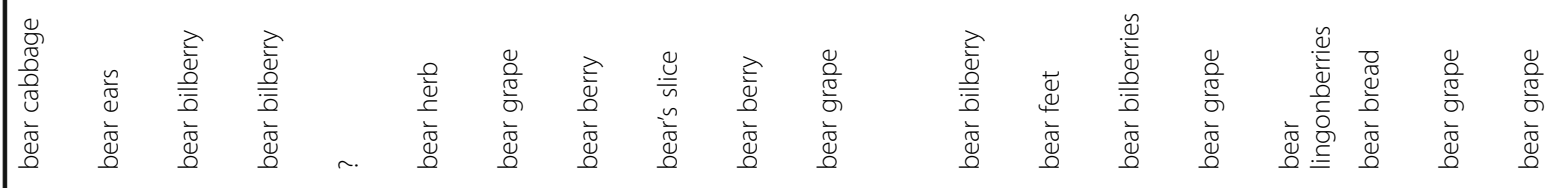

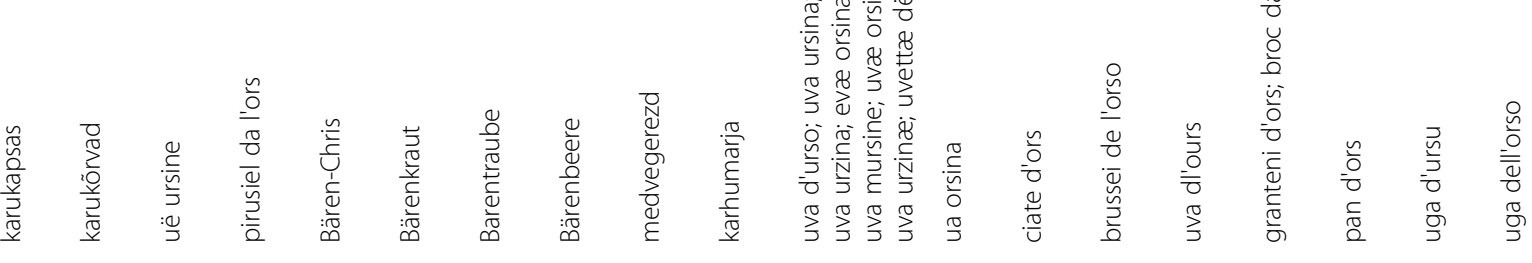

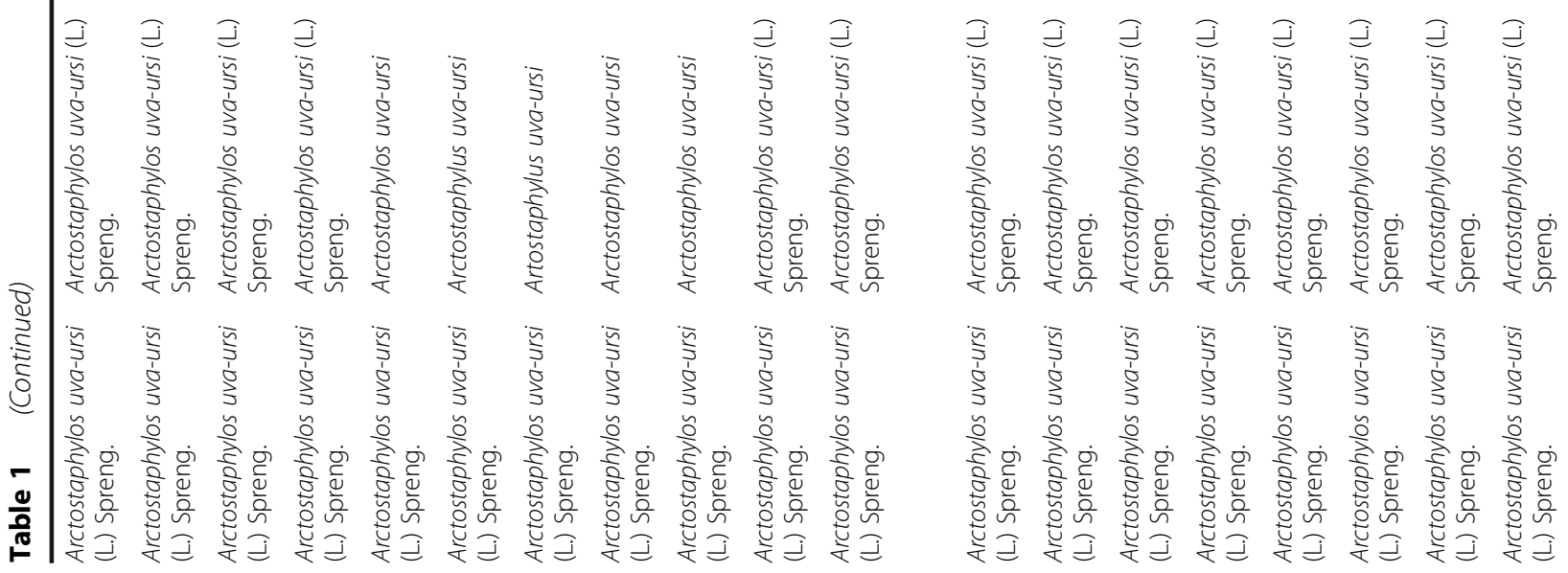




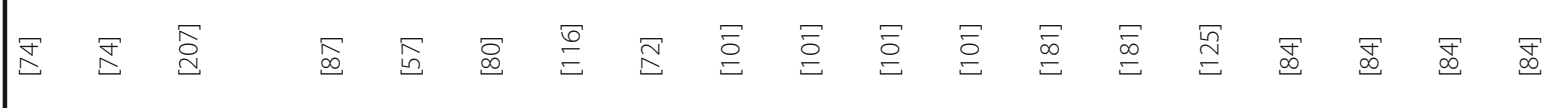

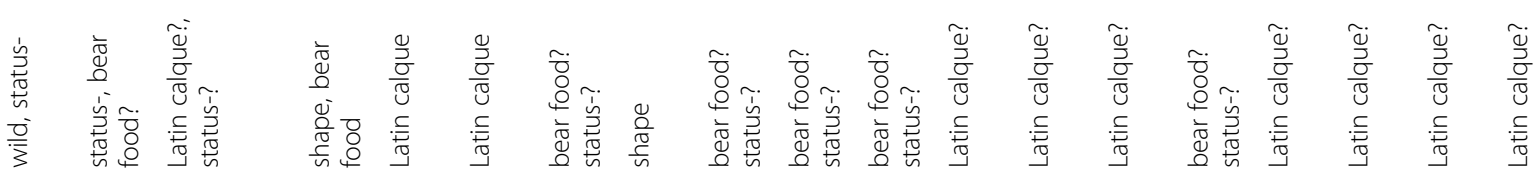

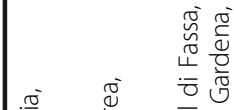

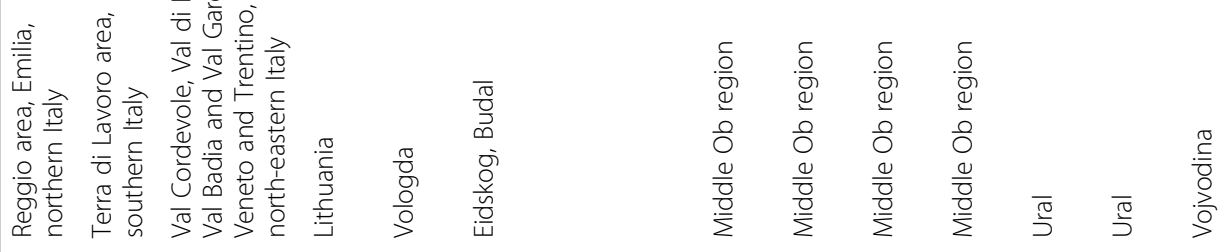

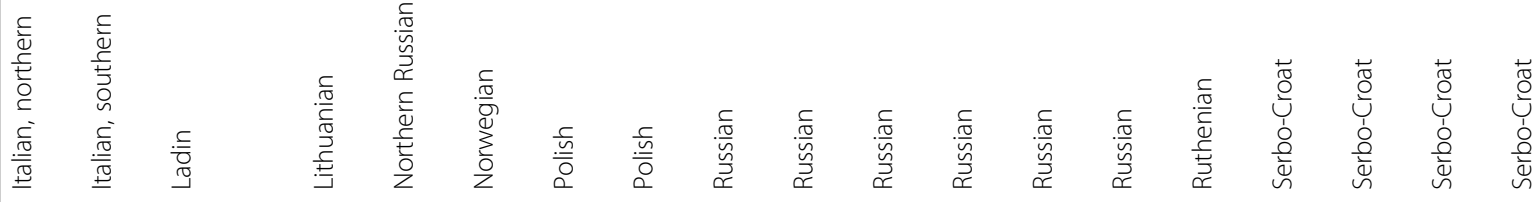

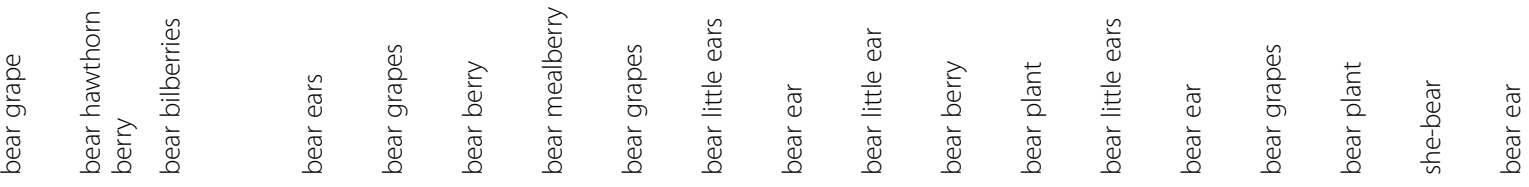

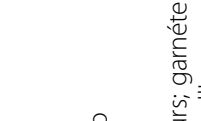

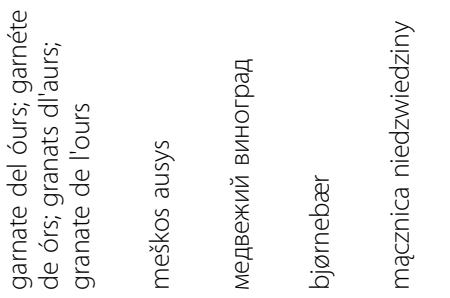

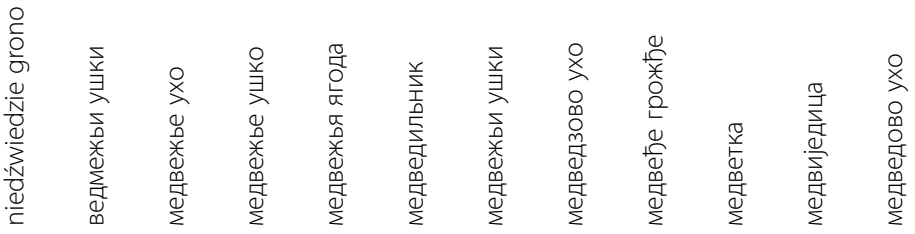

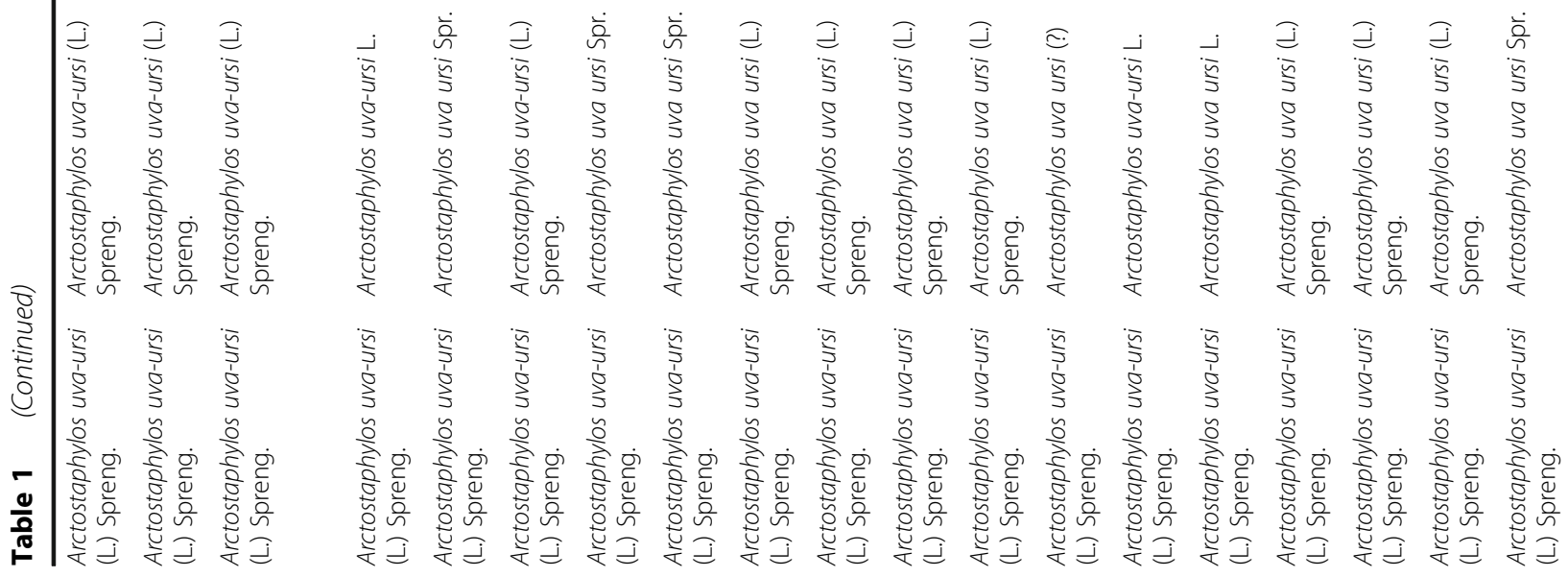




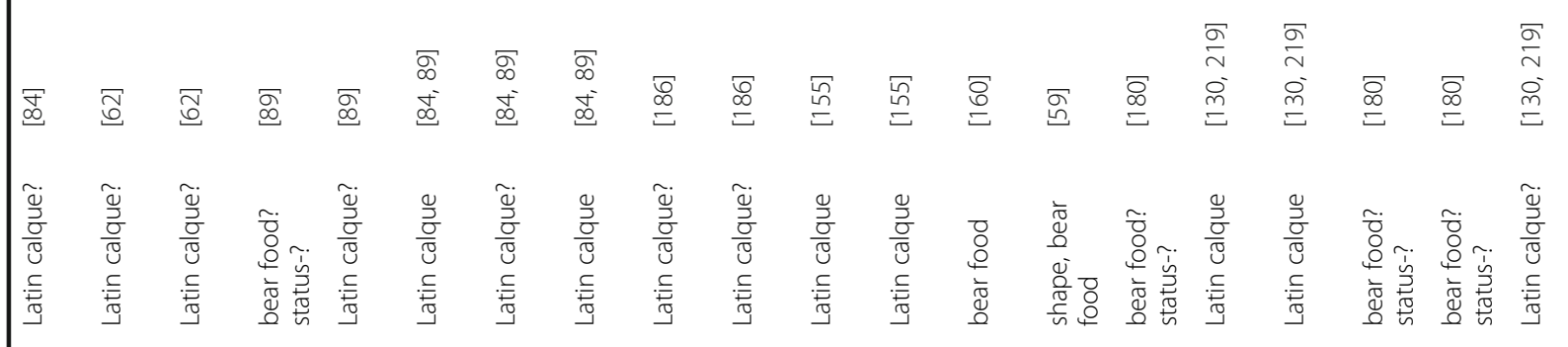

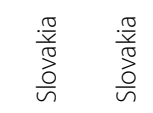

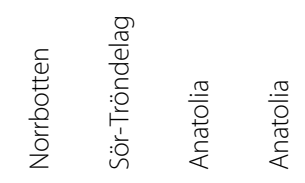

$\frac{\sqrt{0}}{\frac{0}{3}}$

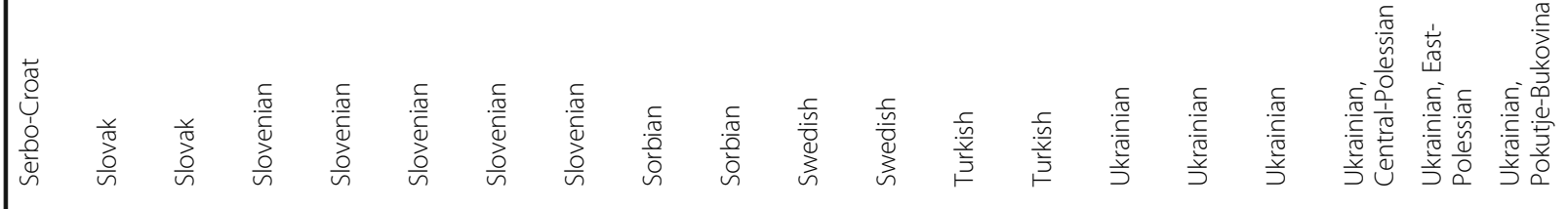

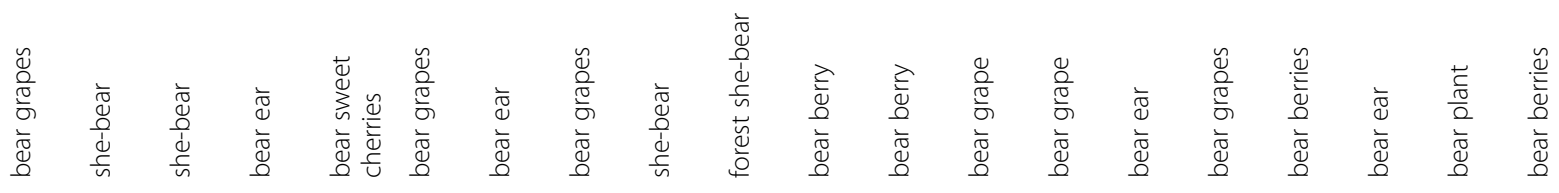

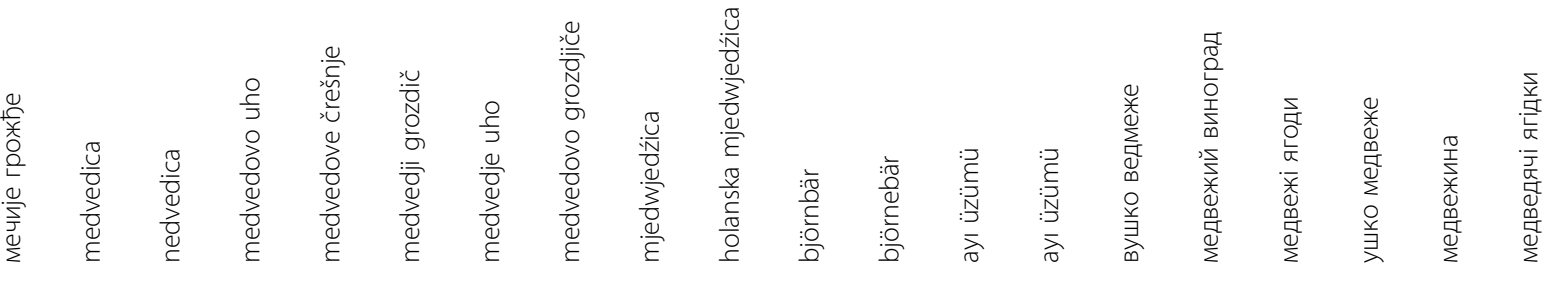

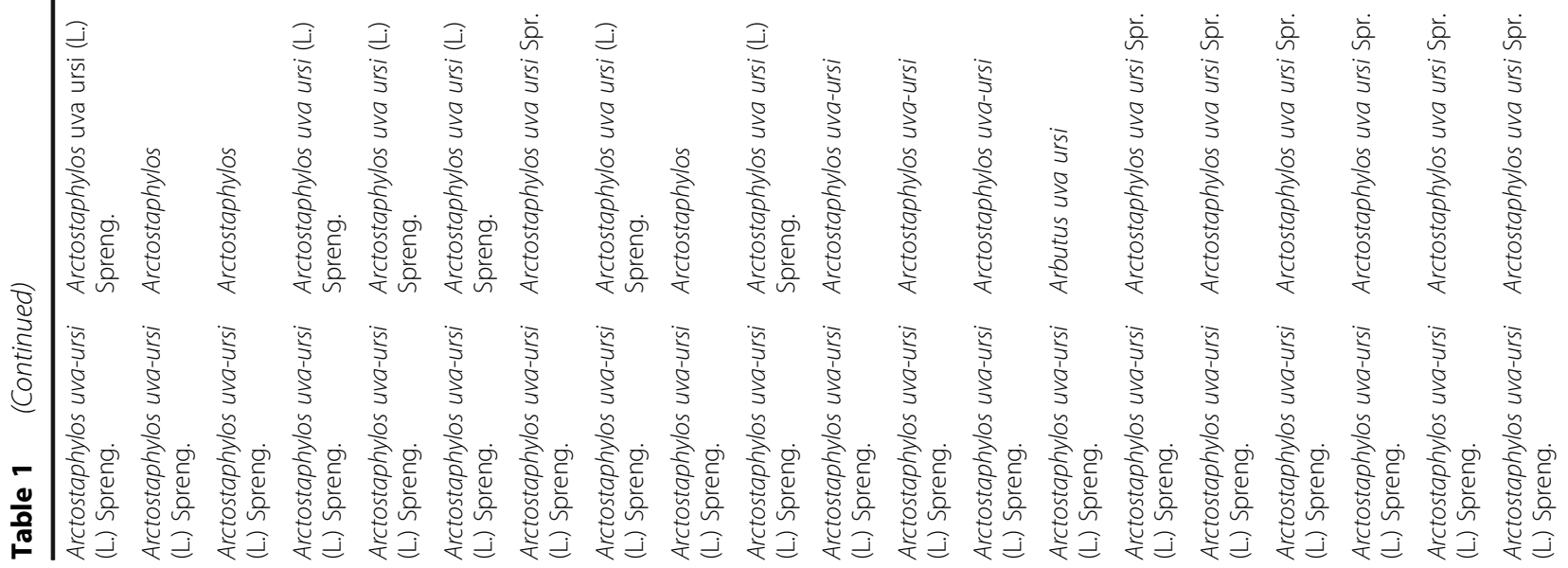




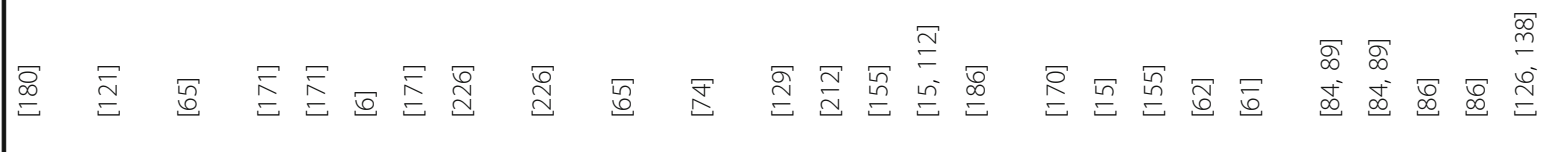

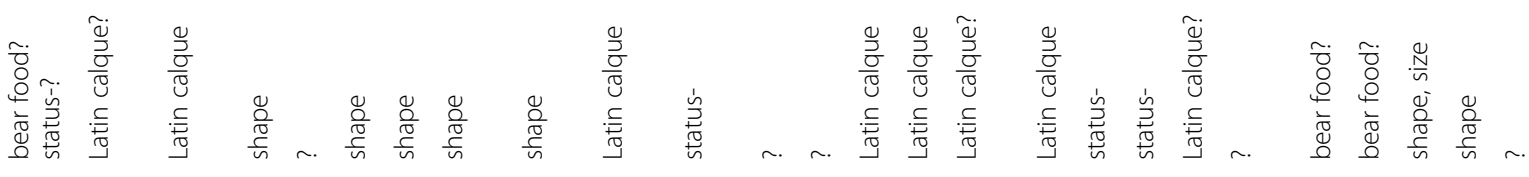

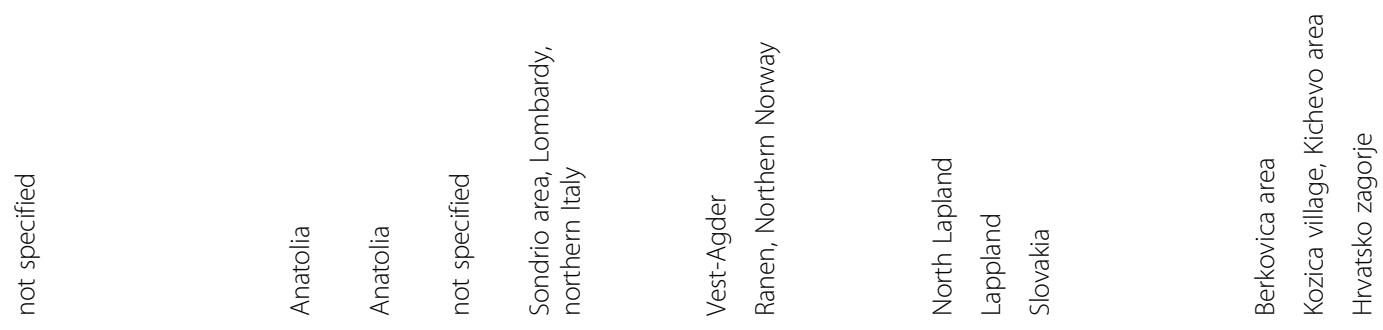

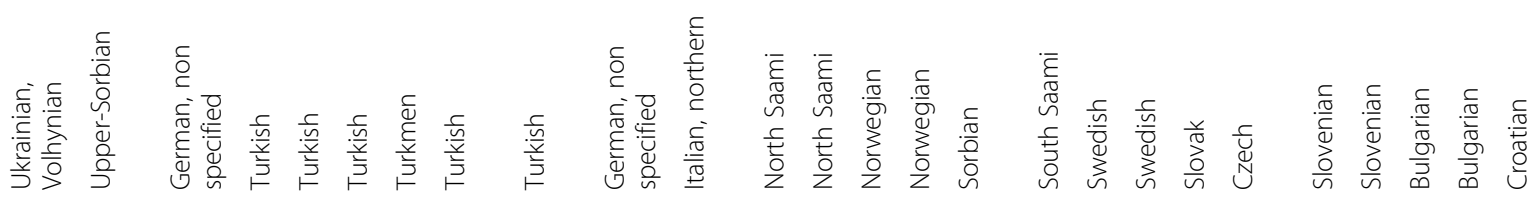

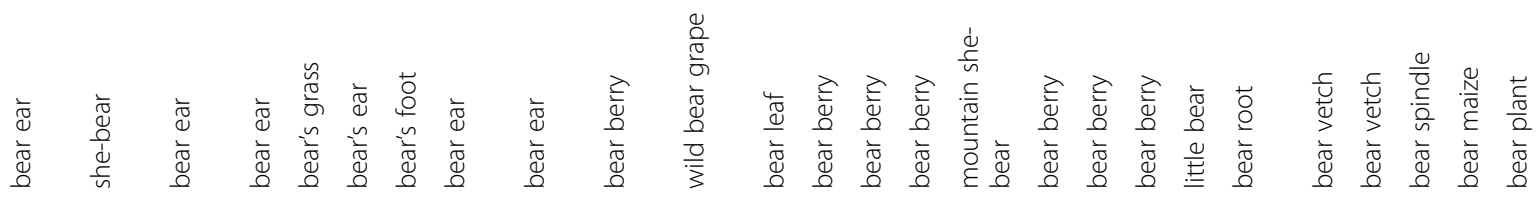

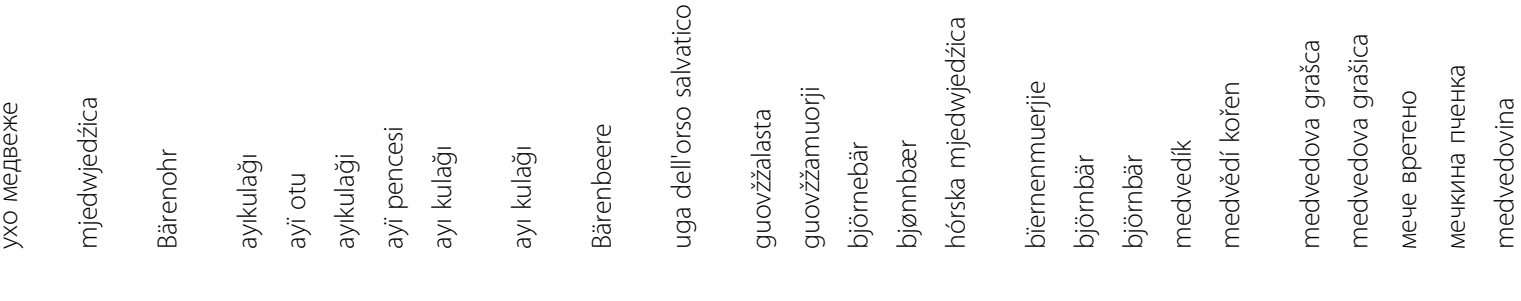

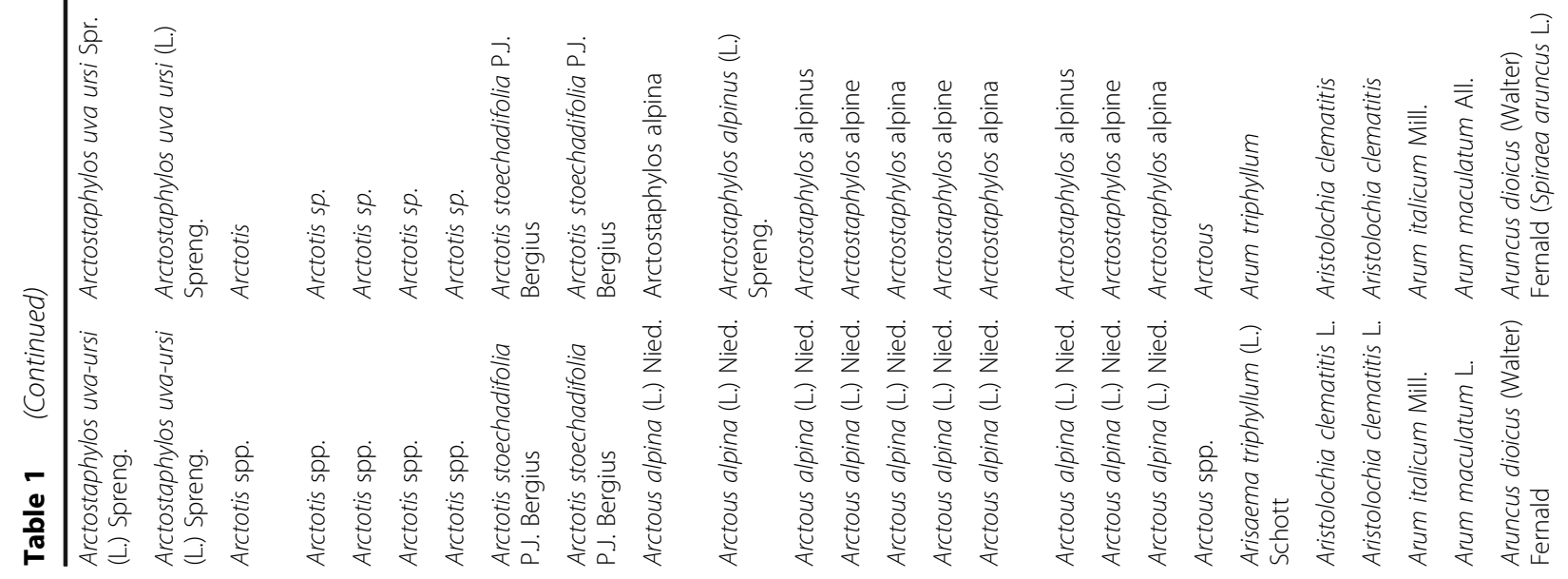




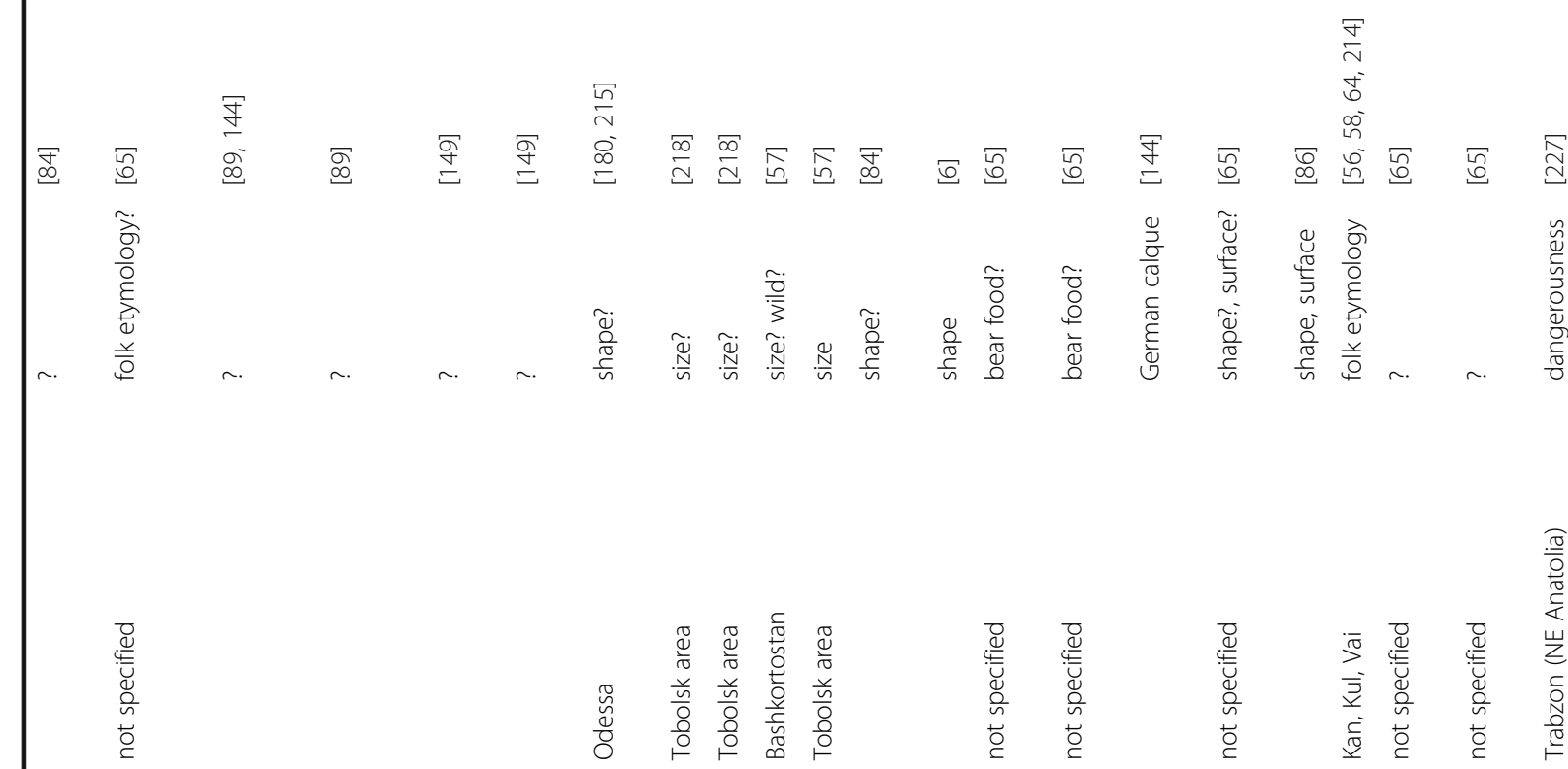

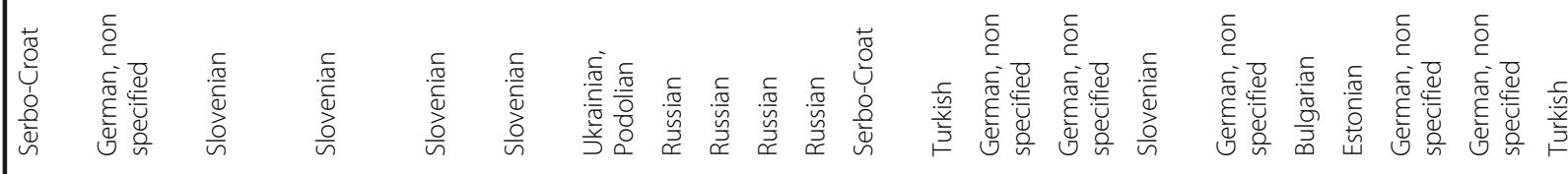

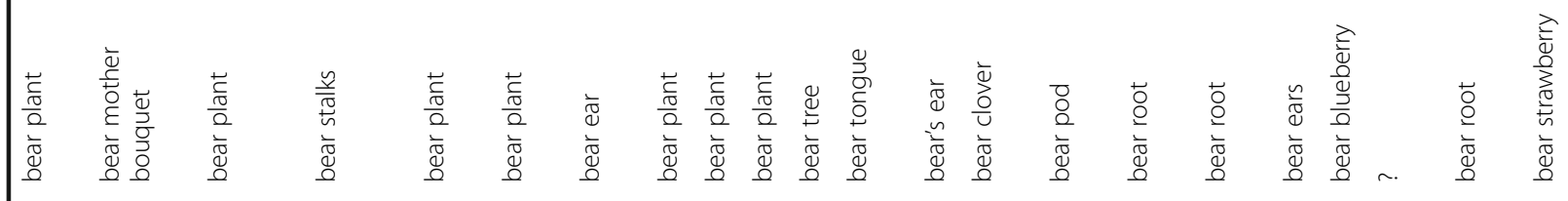

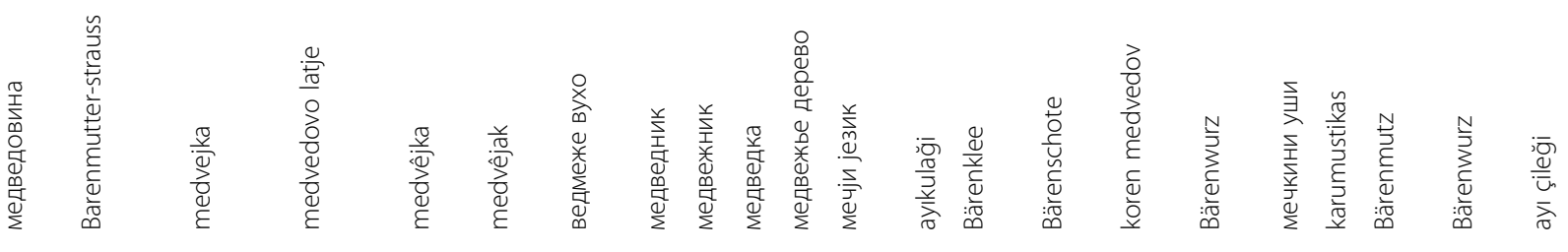<smiles>[CH][CH][CH][CH]</smiles>

$\sqrt{2}$ 


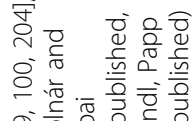

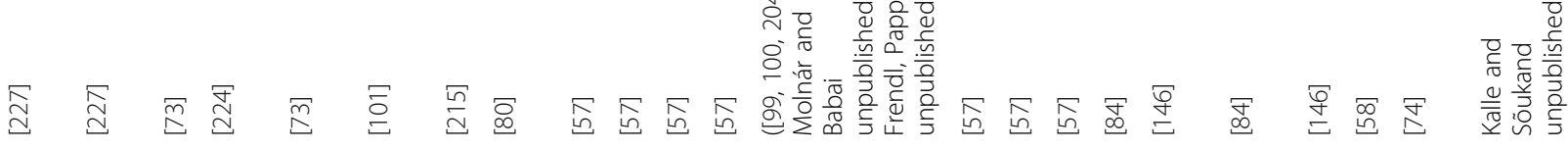

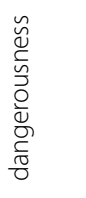

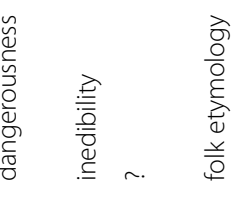

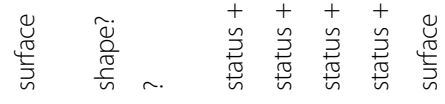

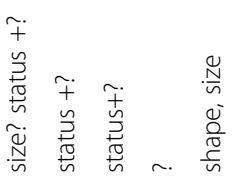

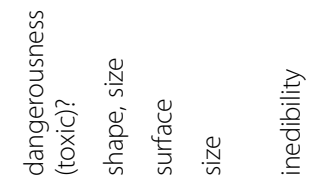

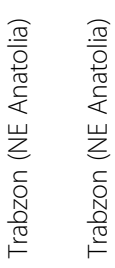

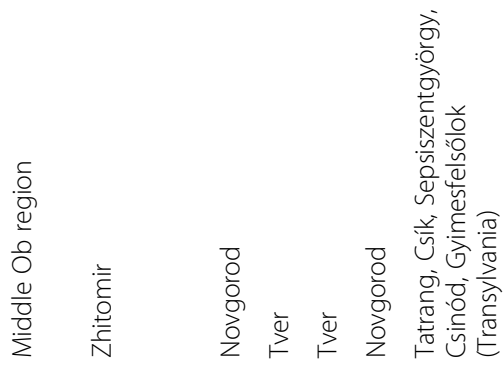

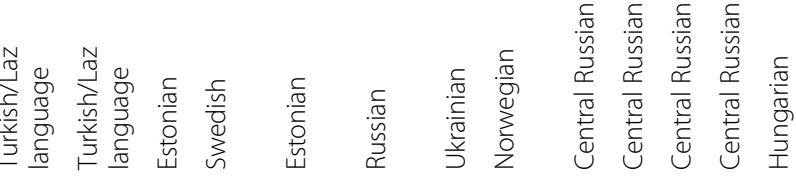

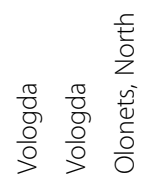<smiles>[CH]1CC1</smiles>

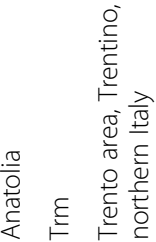

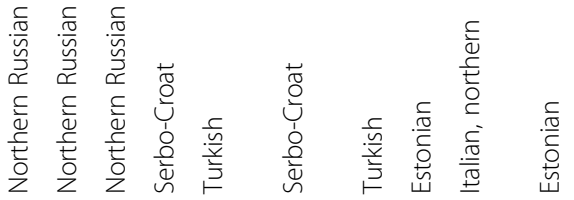

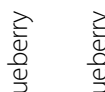

产

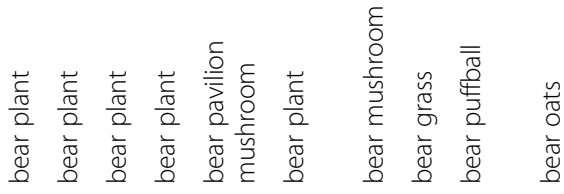

$\begin{array}{llllllllllll} & & & & & & \\ 0\end{array}$

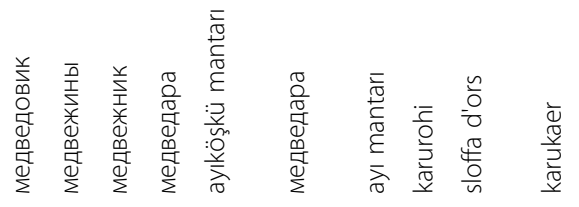

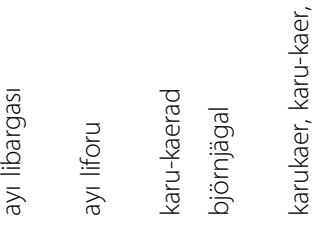

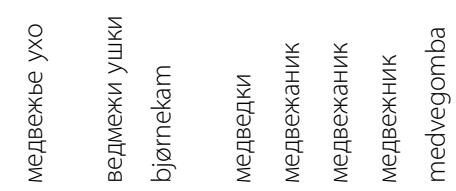

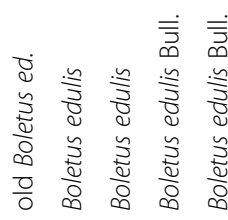

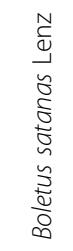

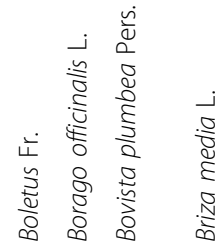




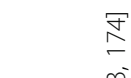

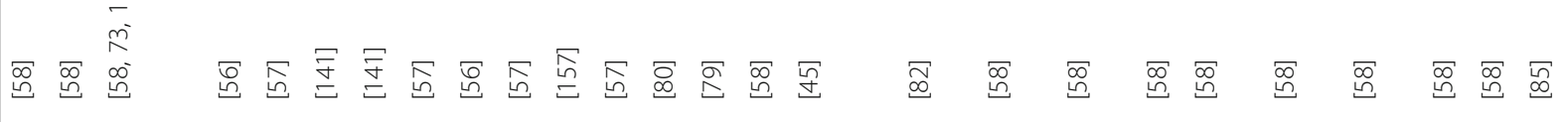

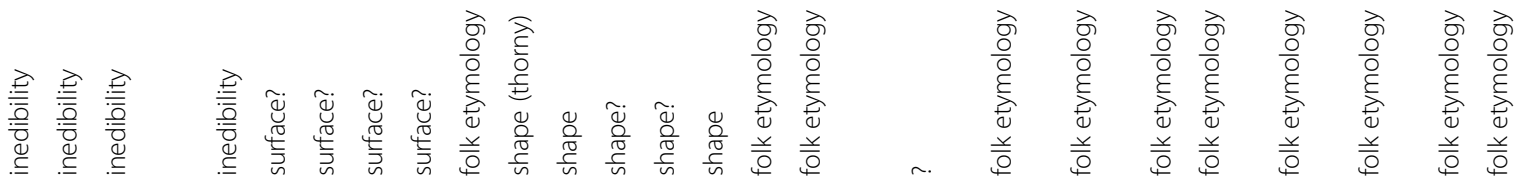

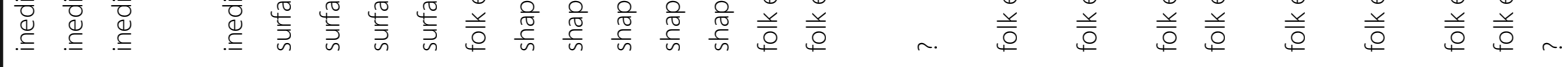

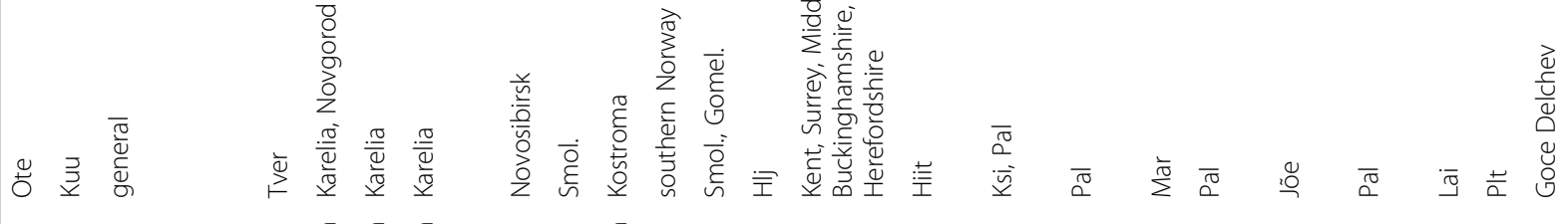

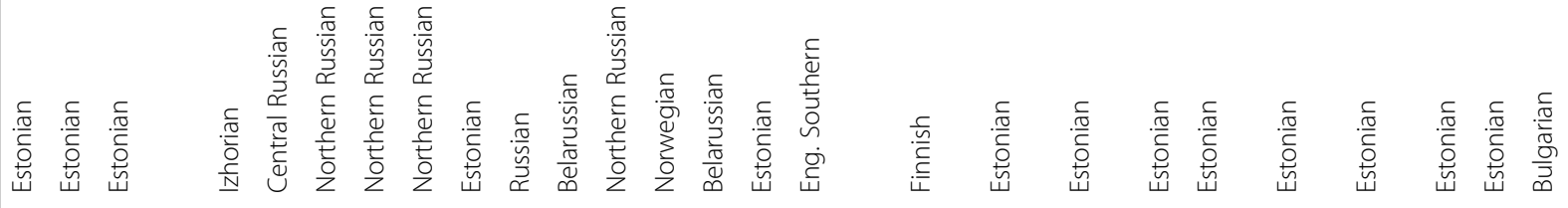

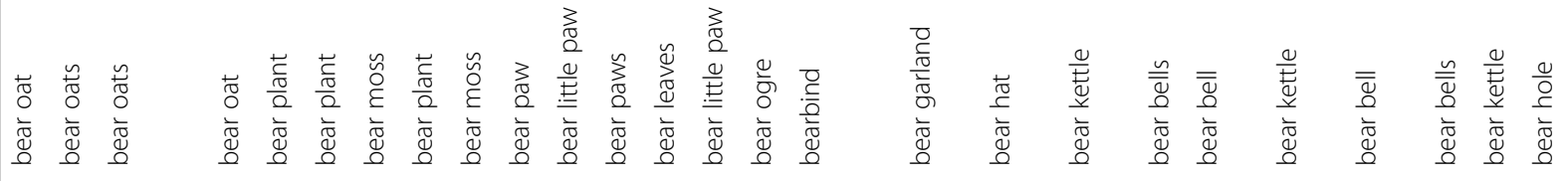

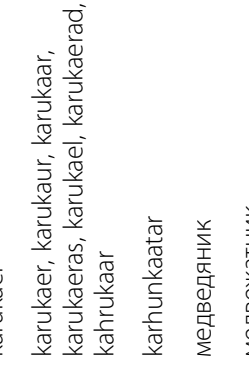

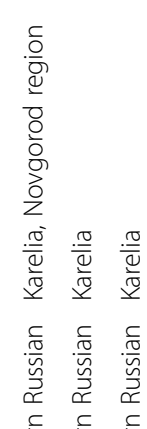

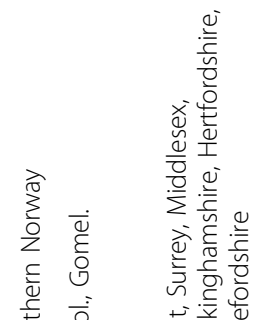




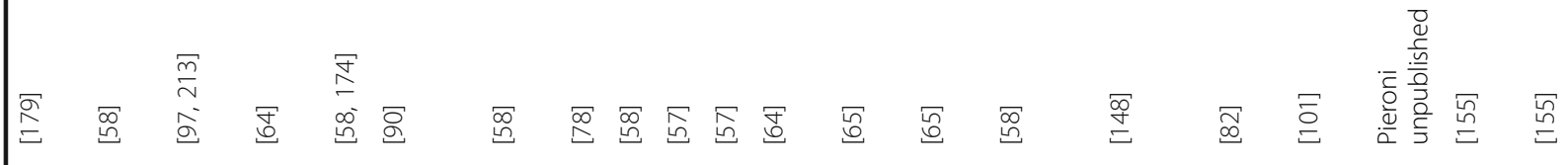

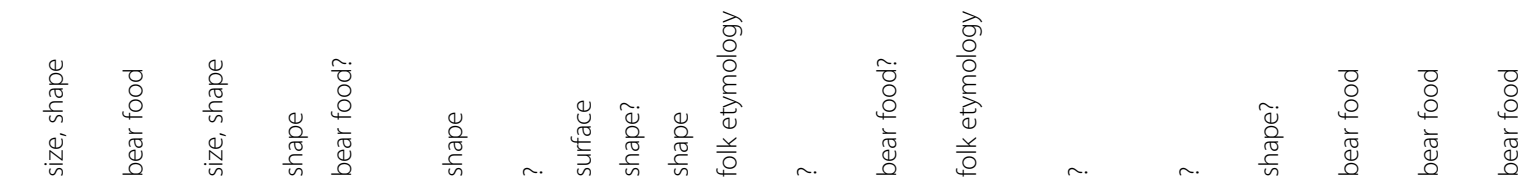

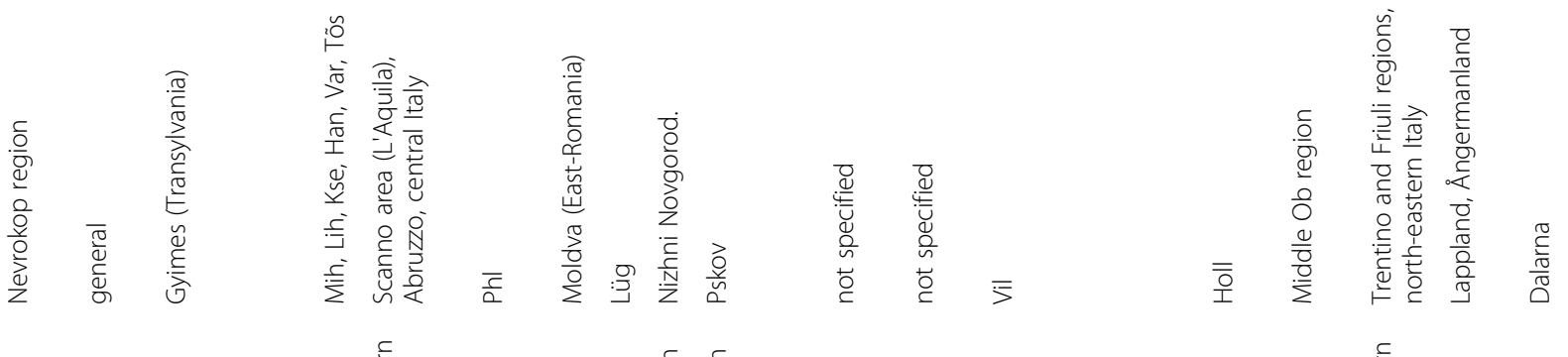

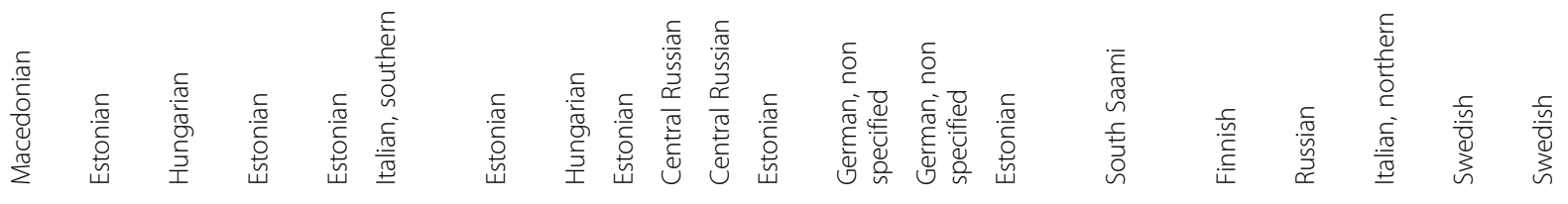

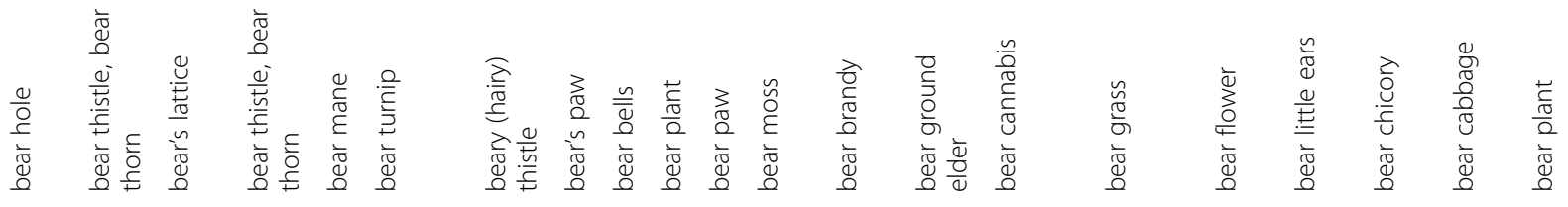

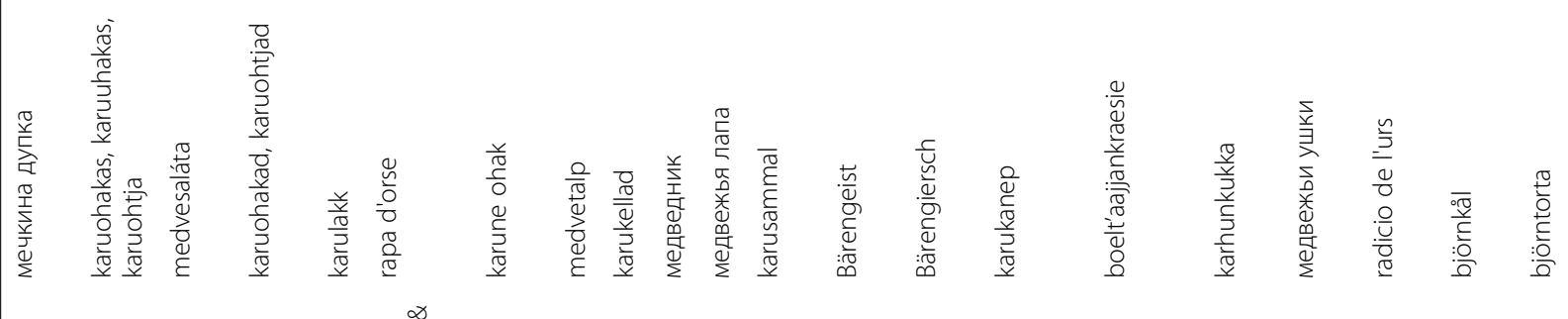

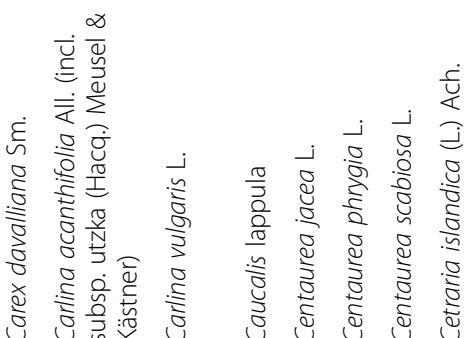

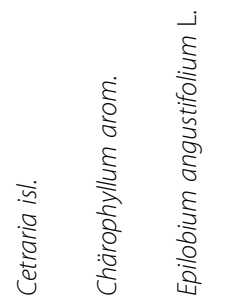

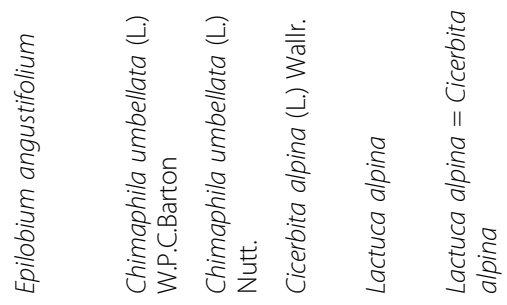

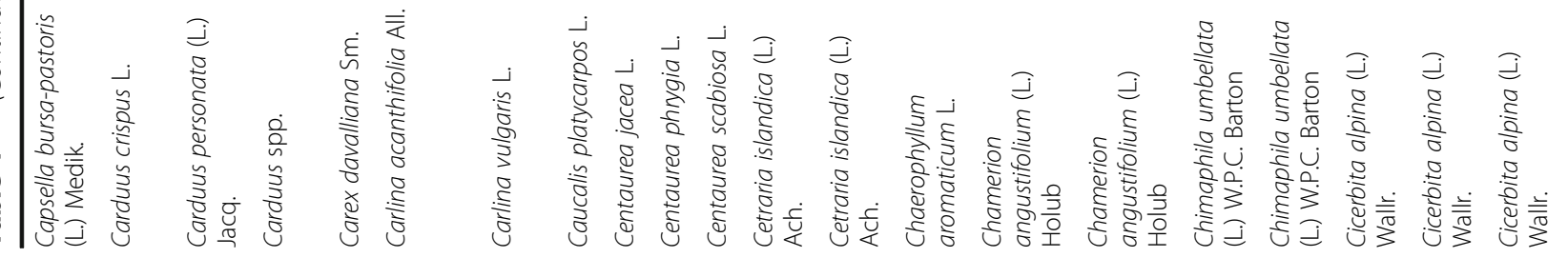




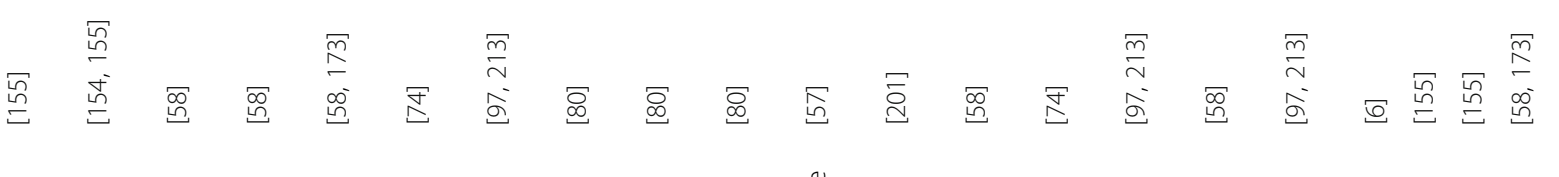

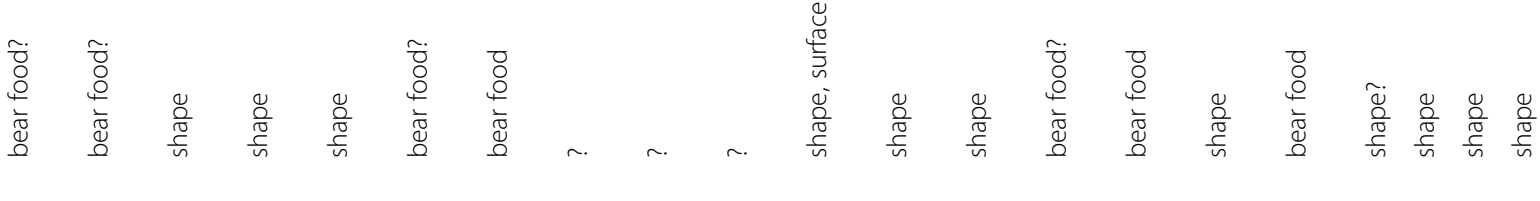

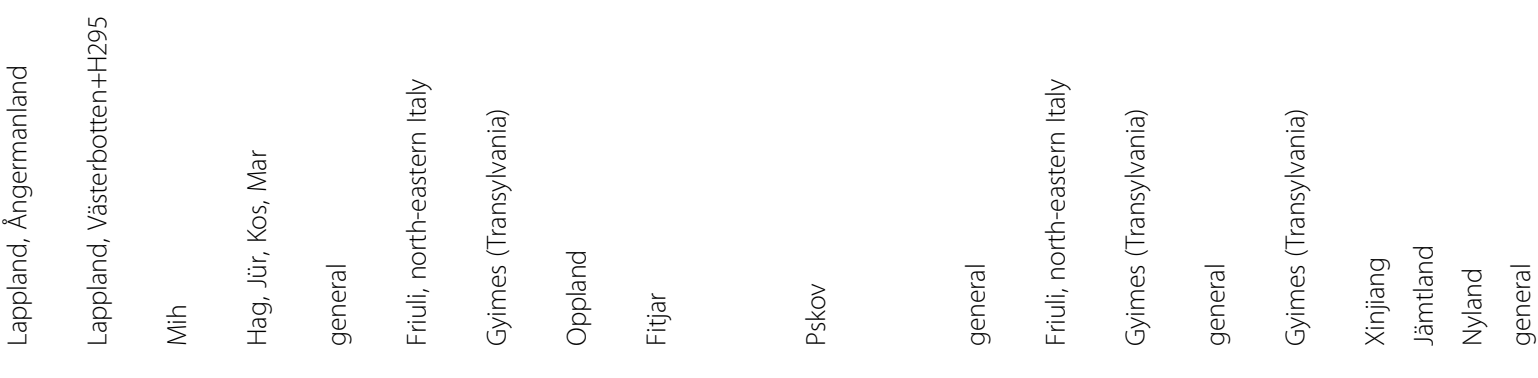

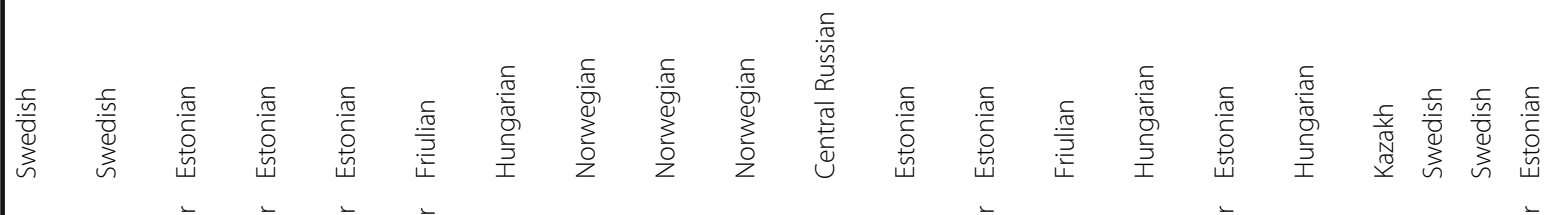

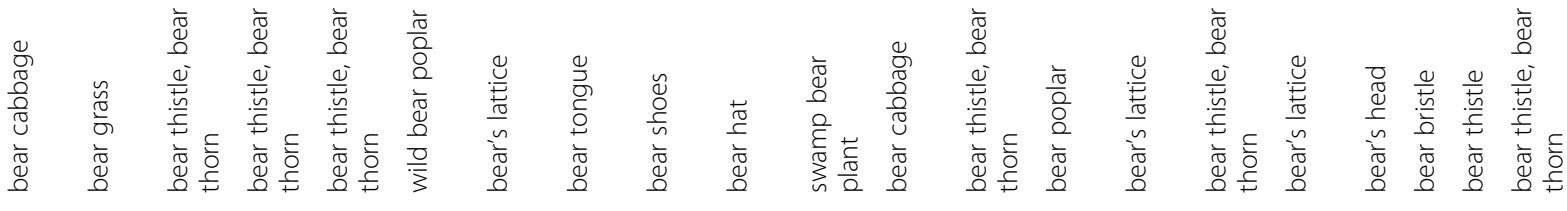

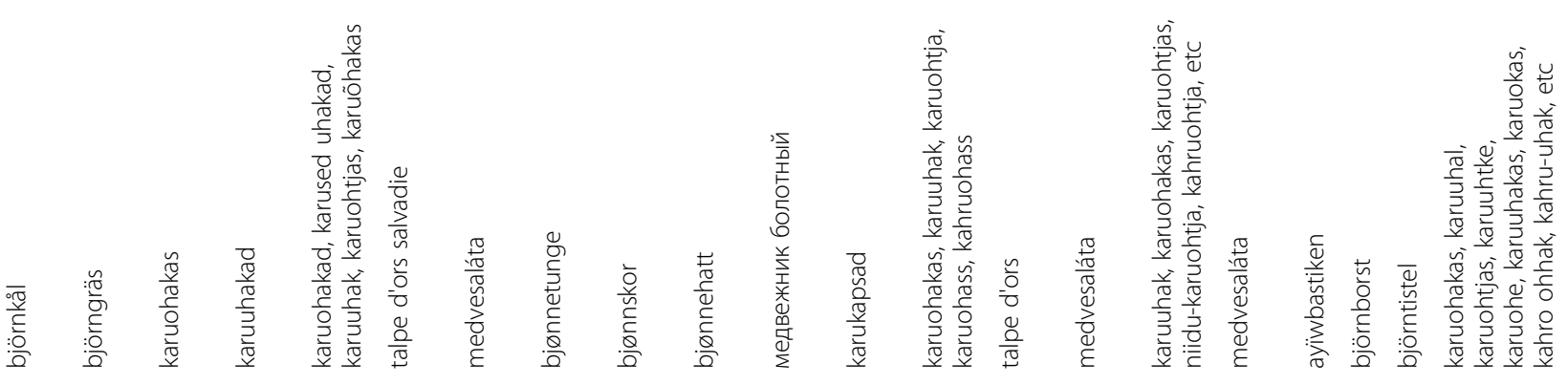

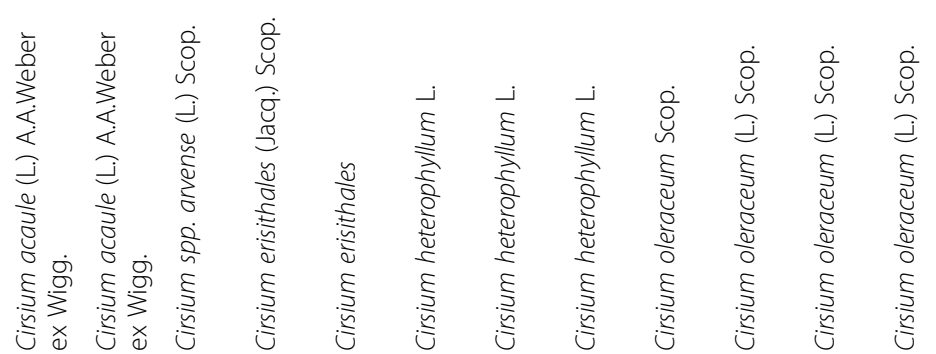

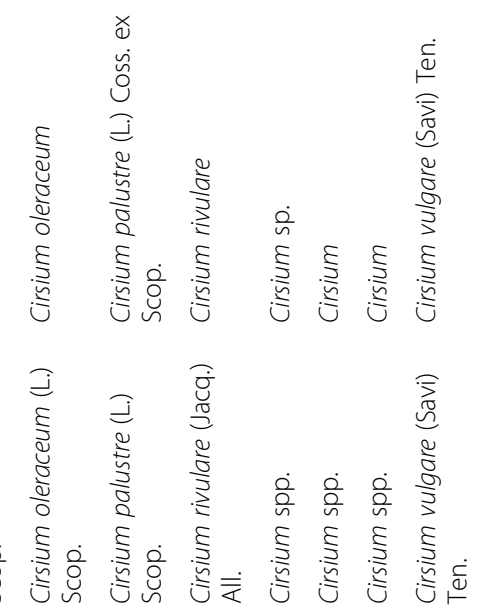




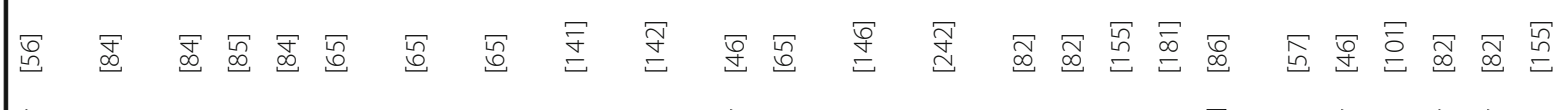

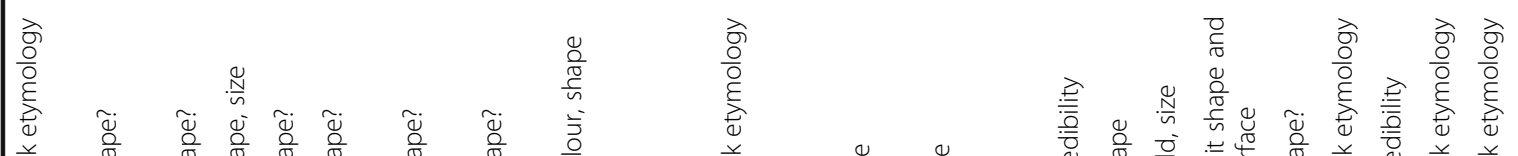

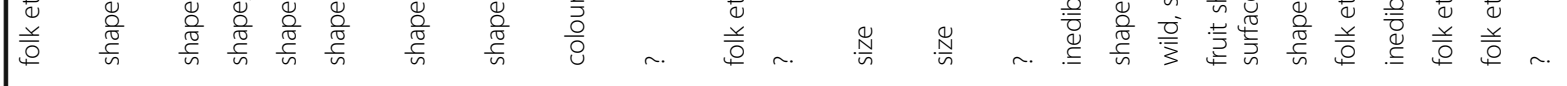

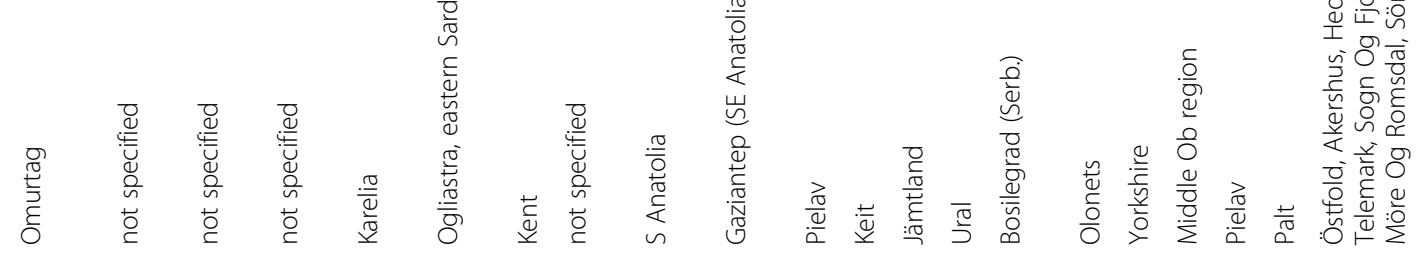

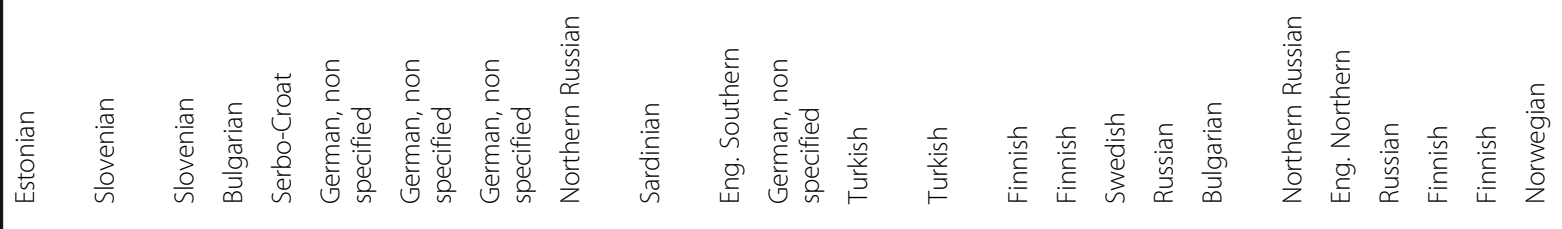

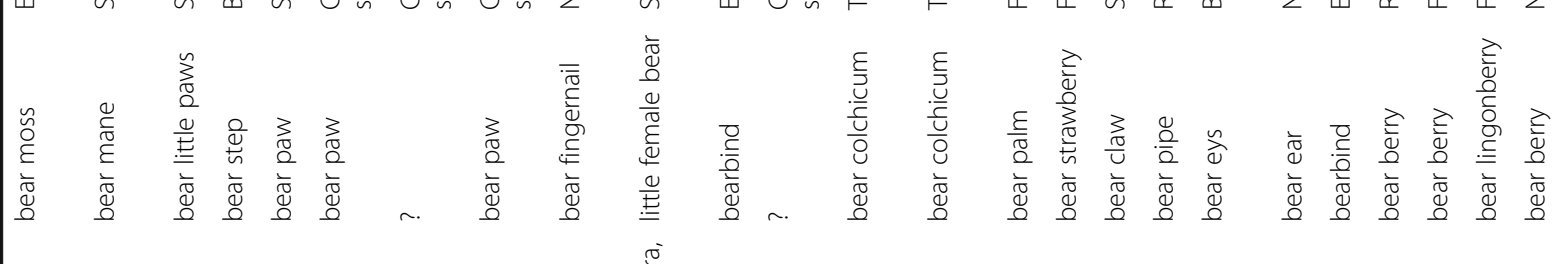

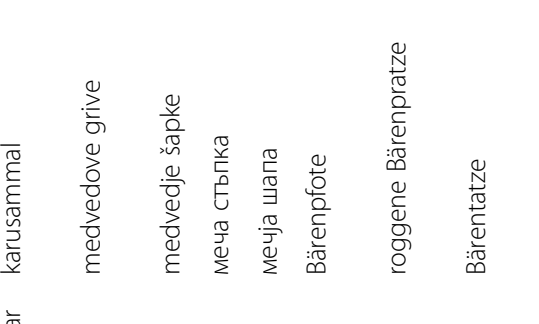

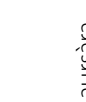

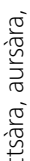

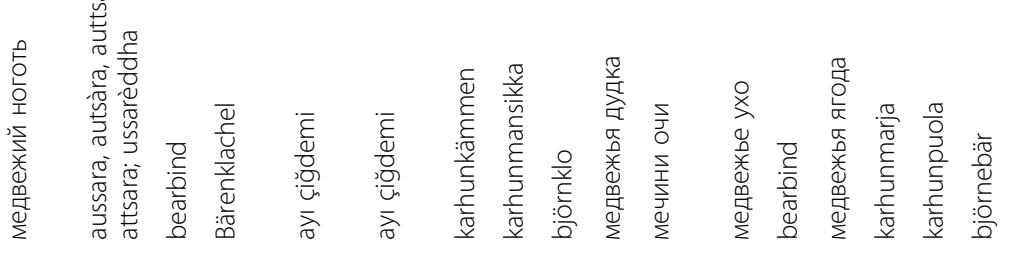

$\sqrt{3}$

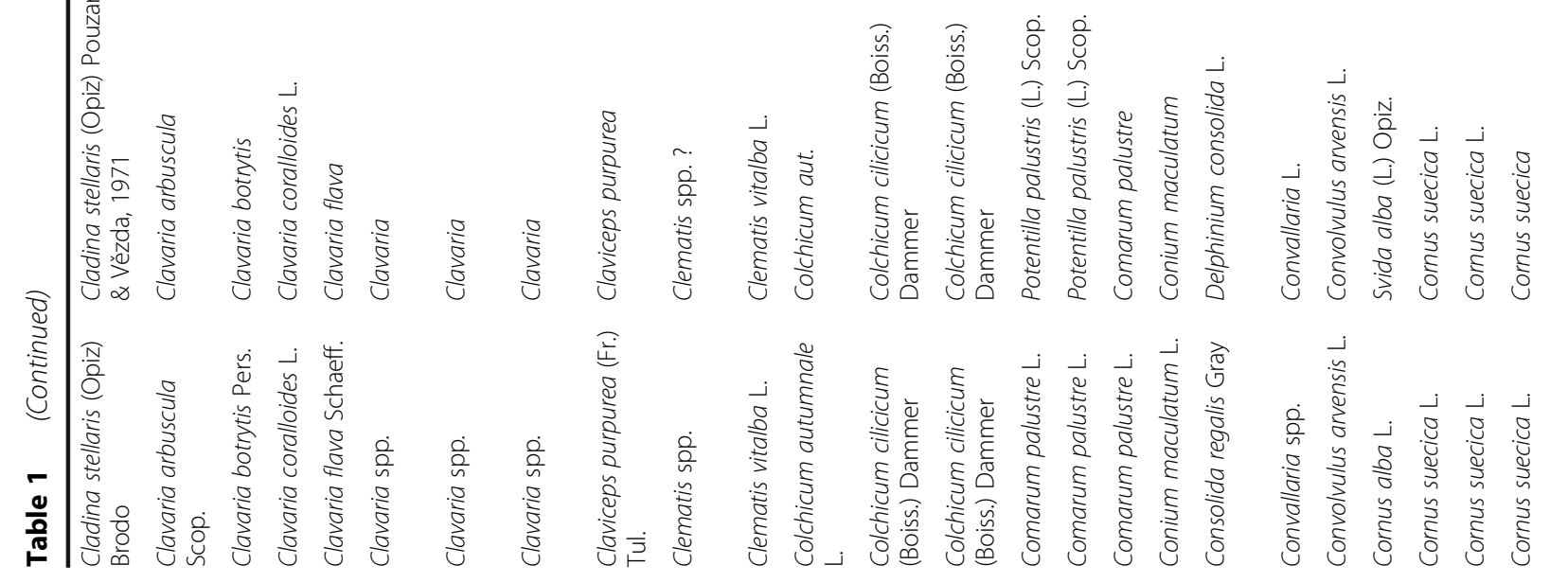




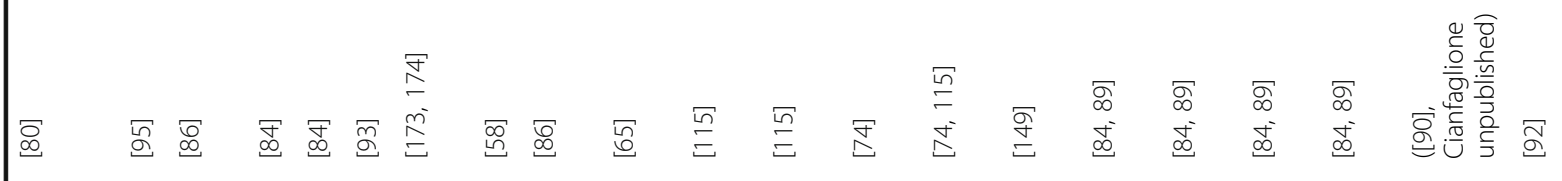

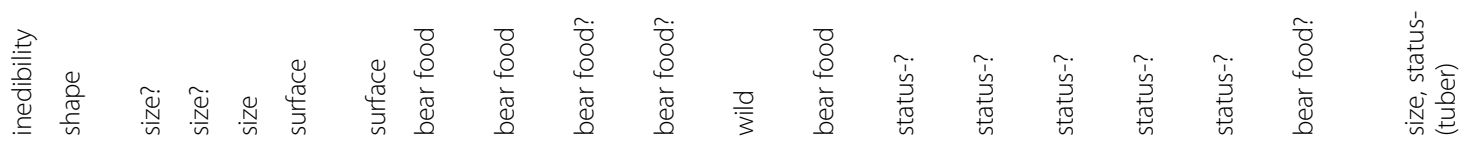

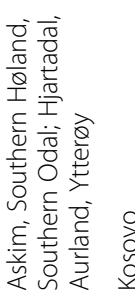
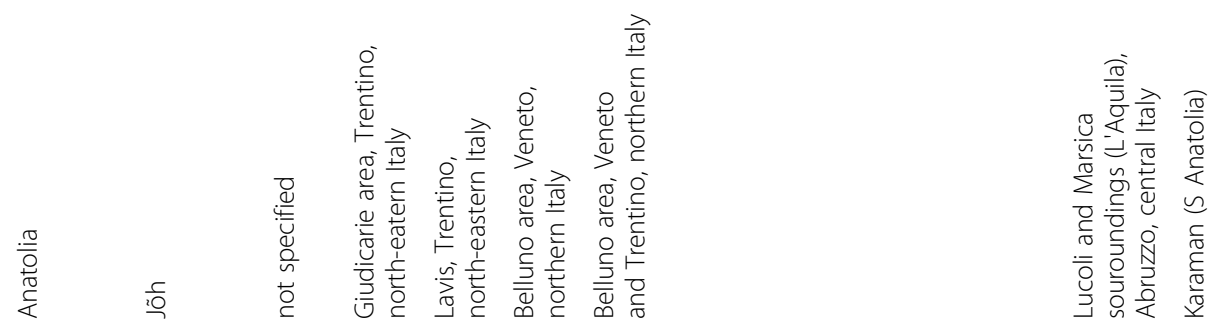

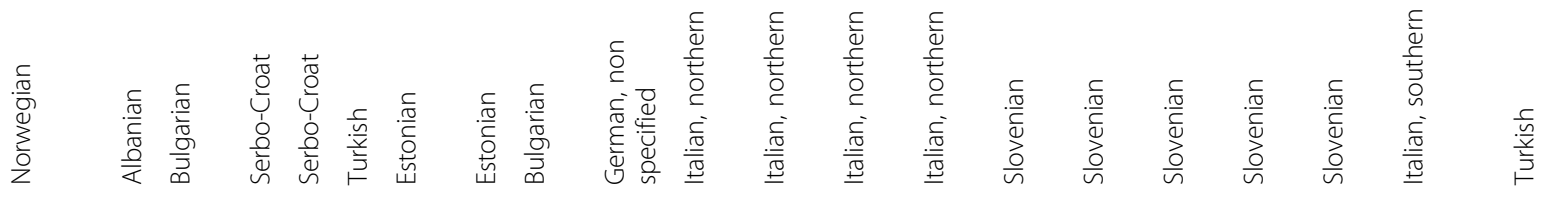

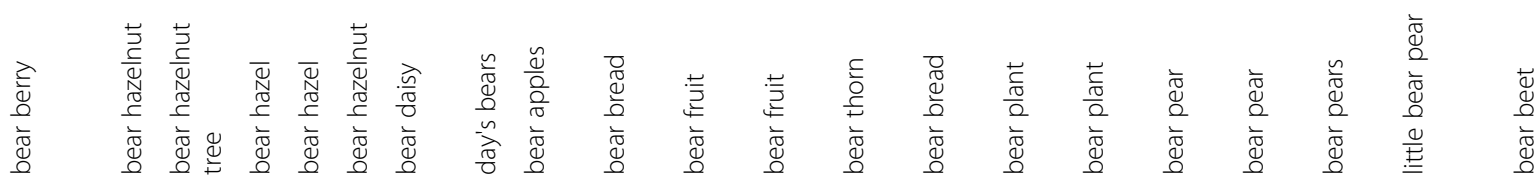

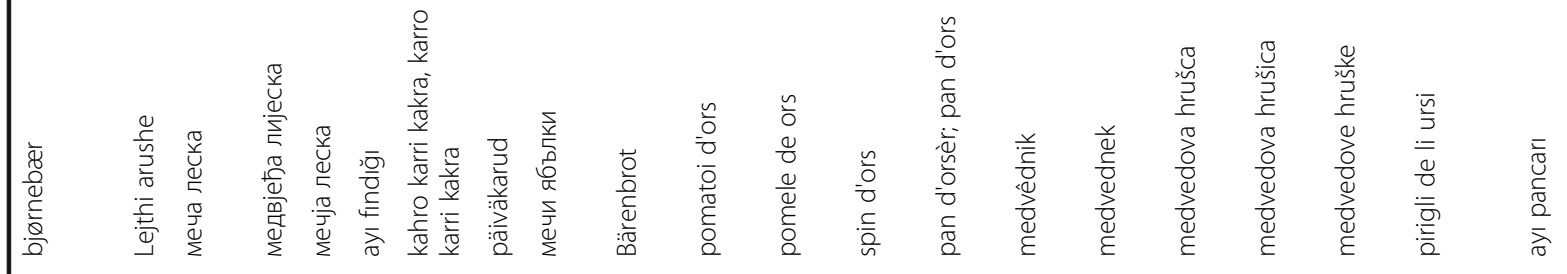




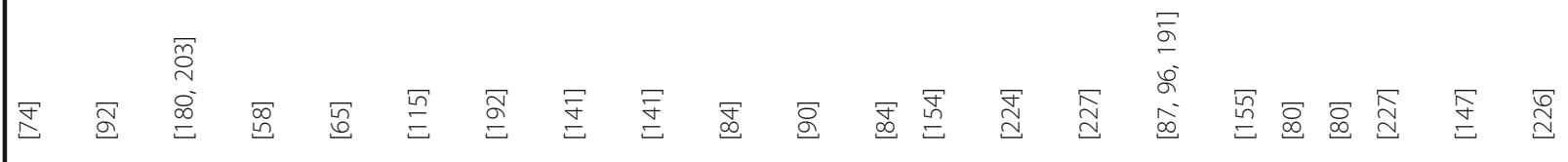

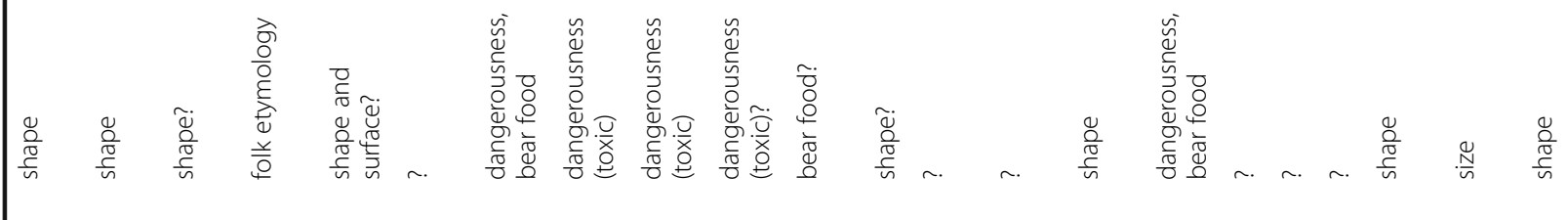

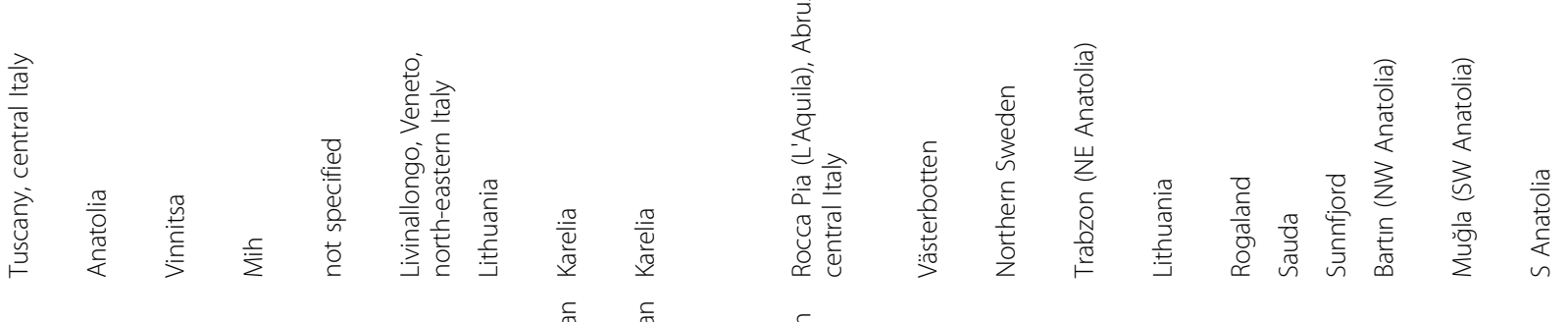

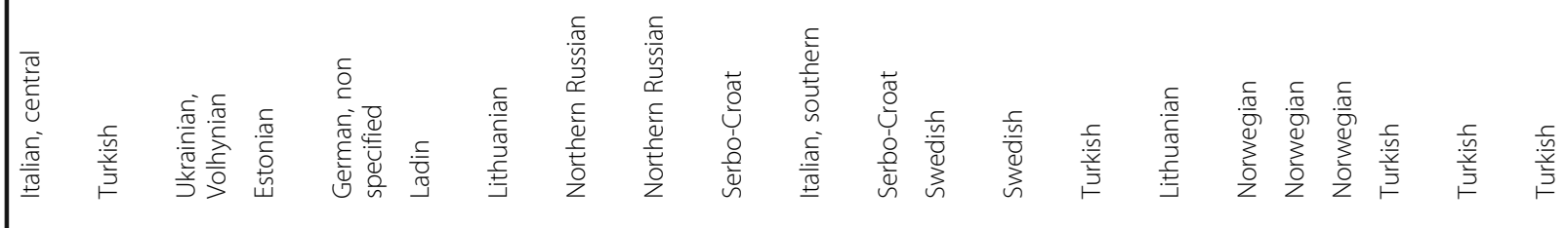

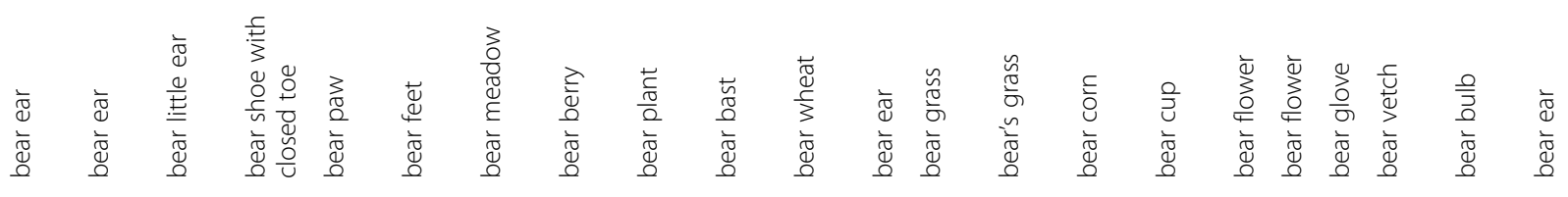

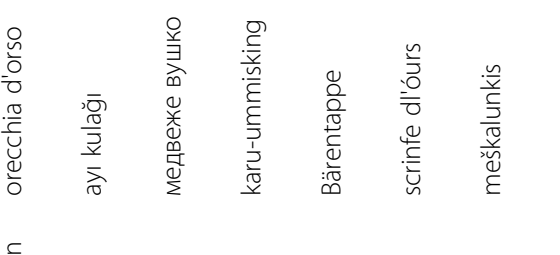

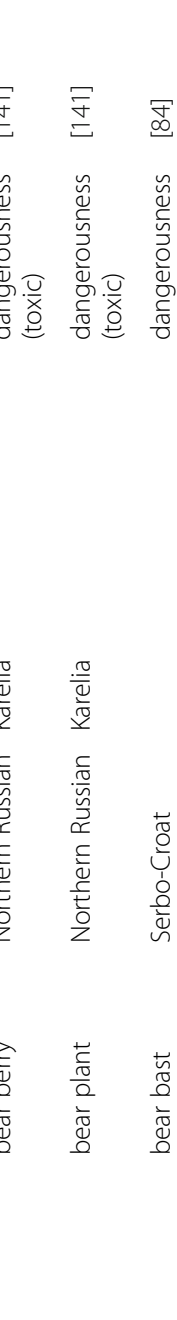

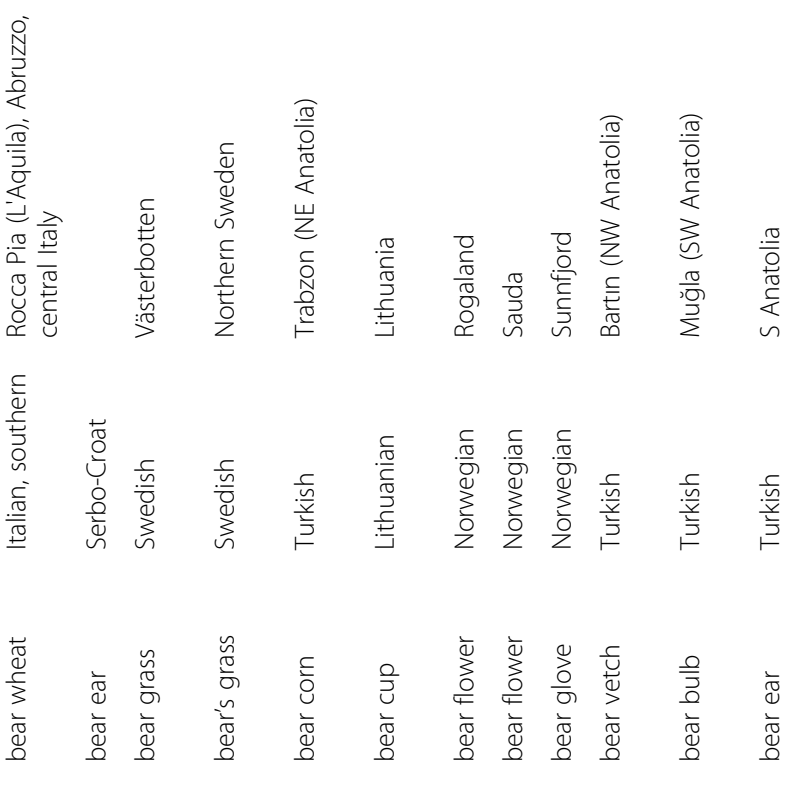

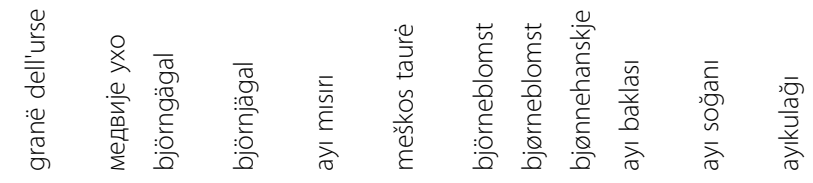

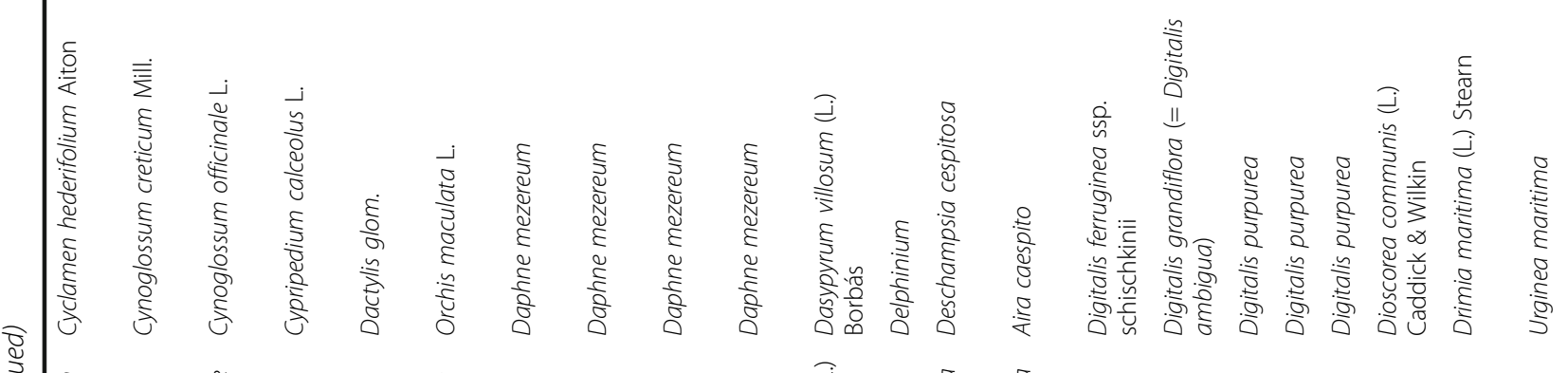

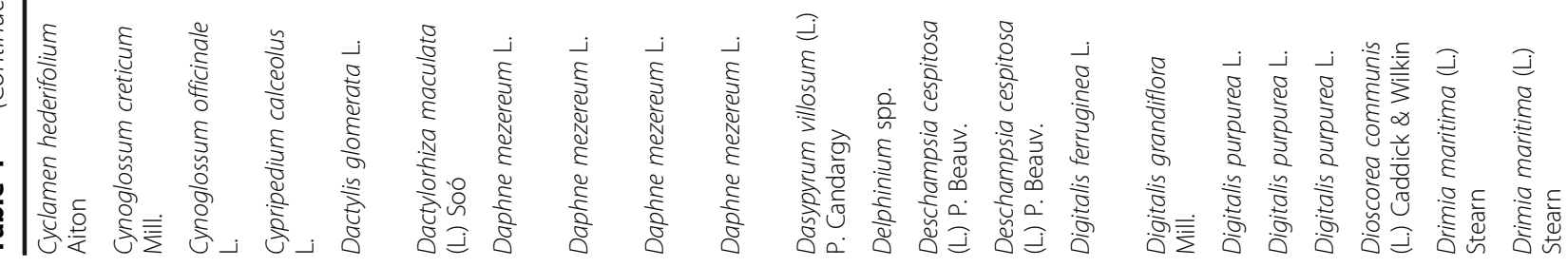




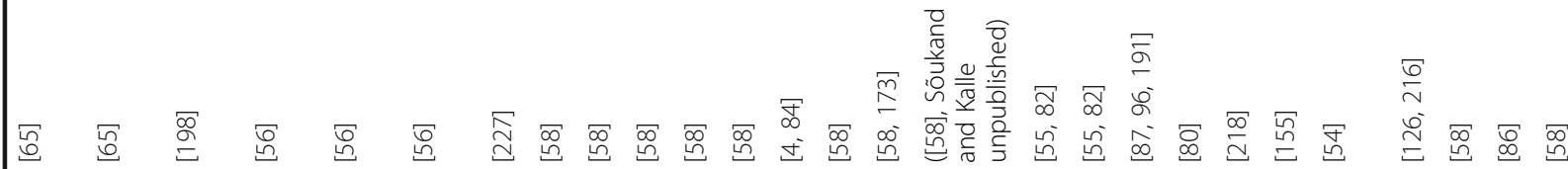

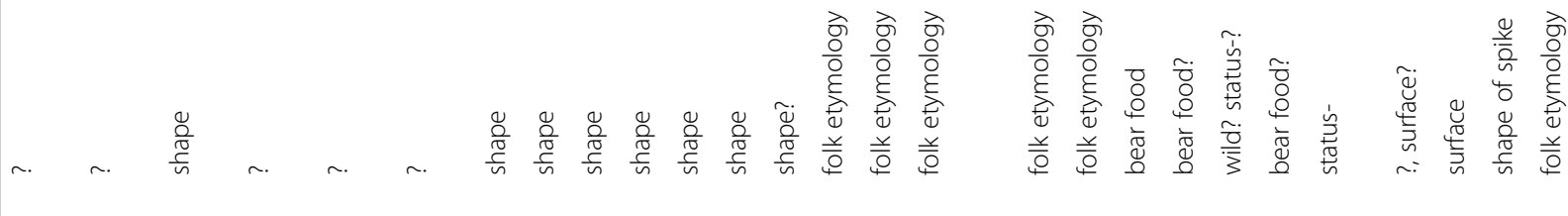

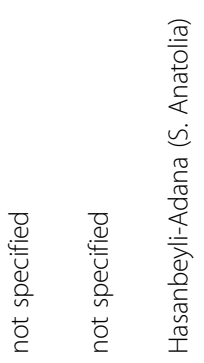

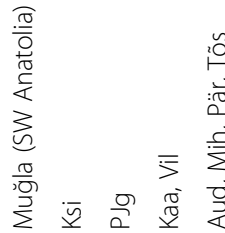

$\stackrel{n}{\stackrel{2}{\circ}}$

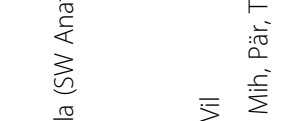

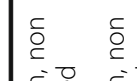

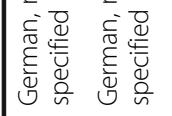

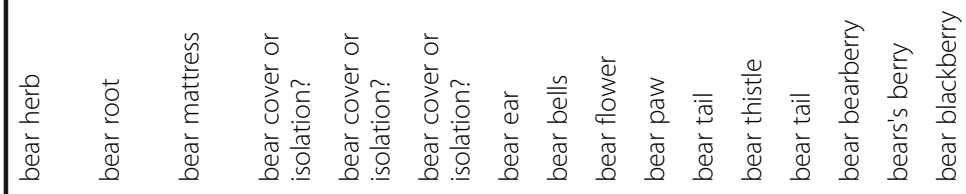

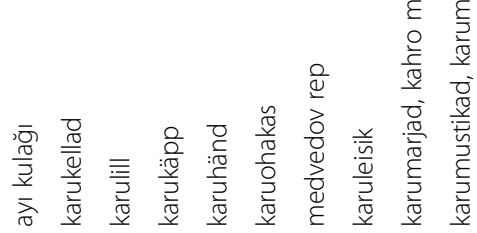

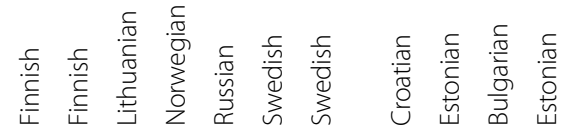

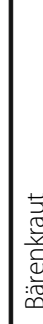

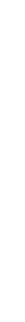

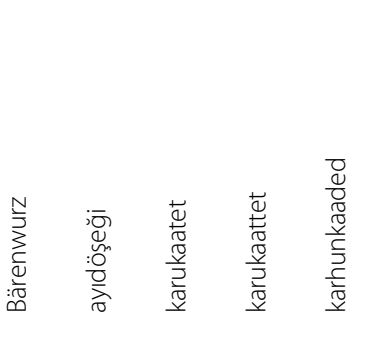

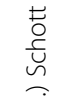

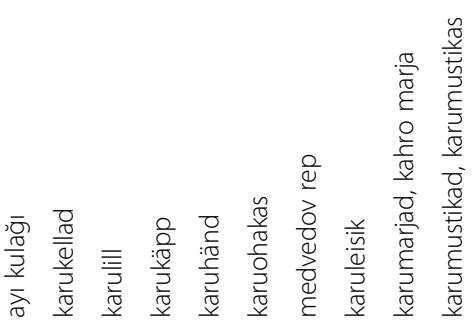

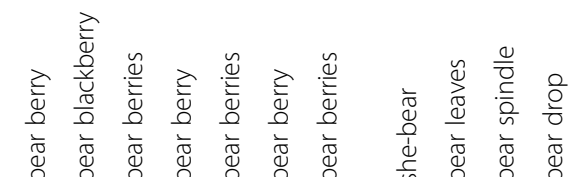

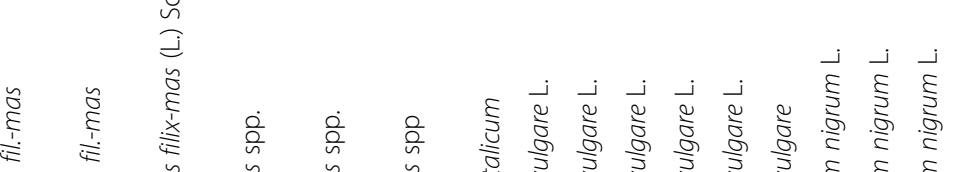

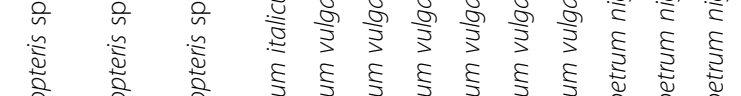

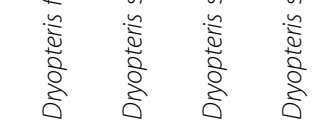

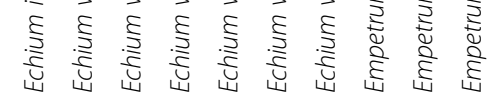

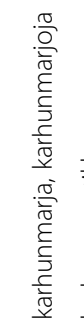

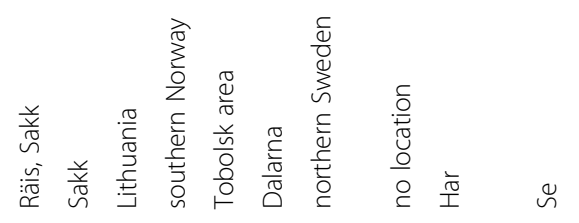

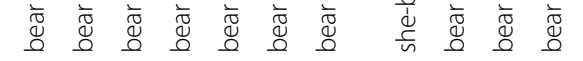
깅 .

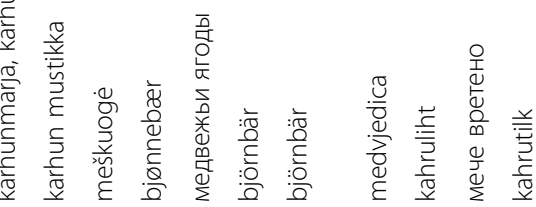

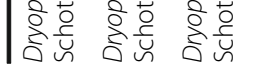
के 


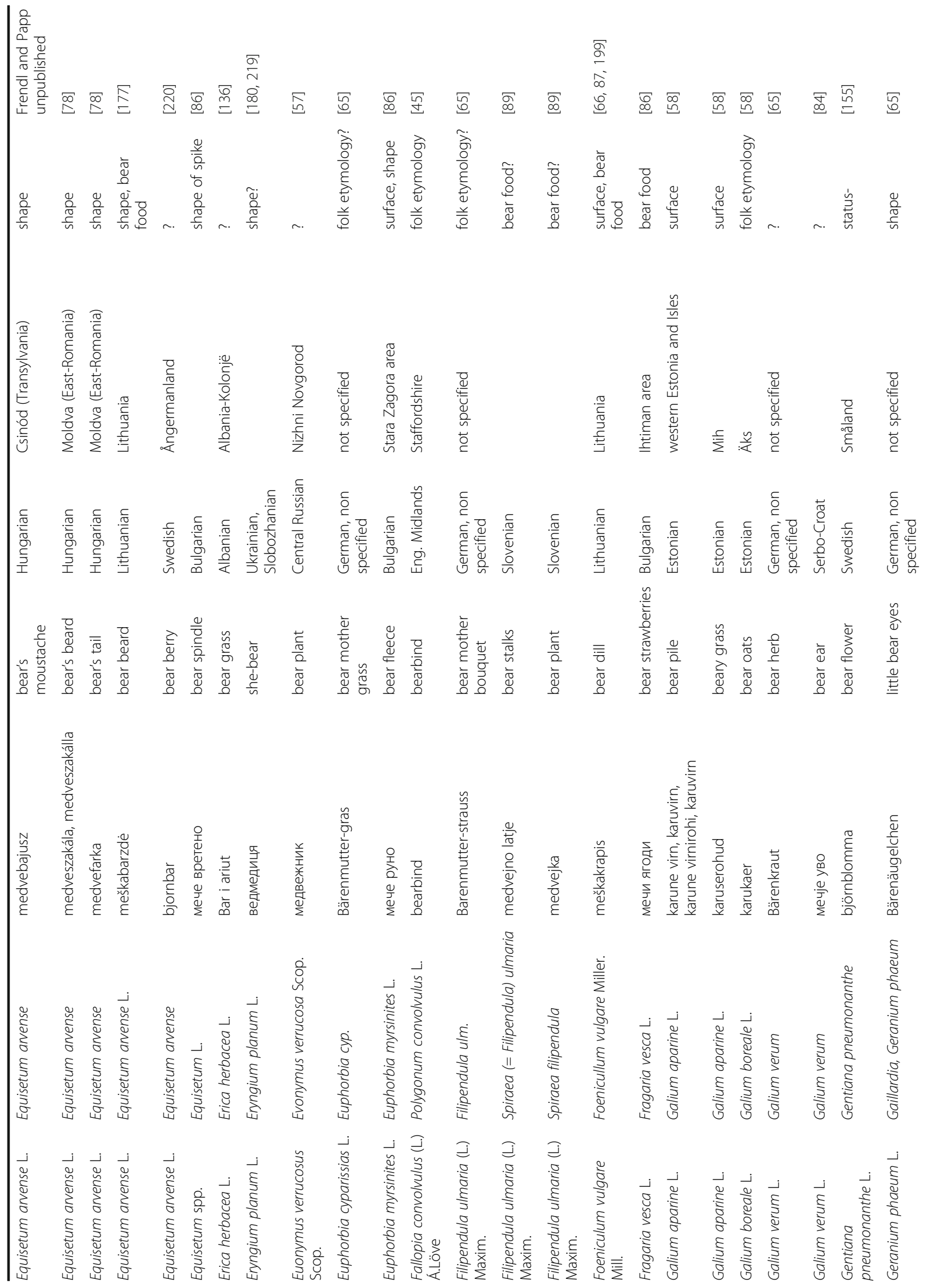




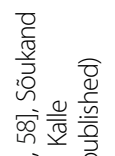

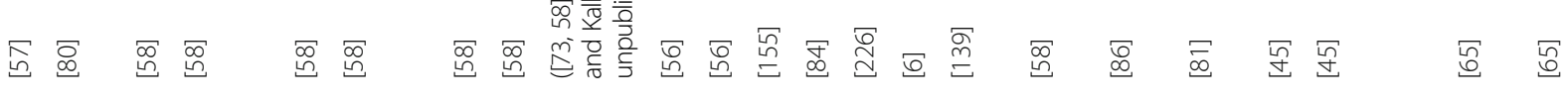

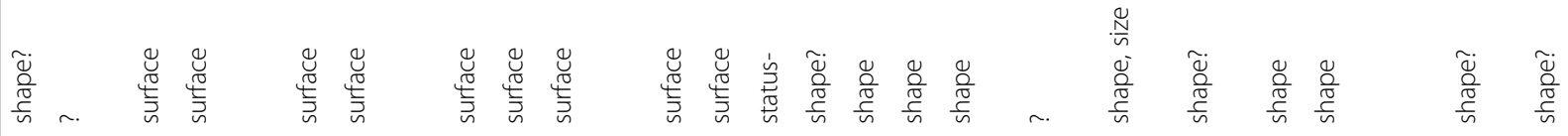

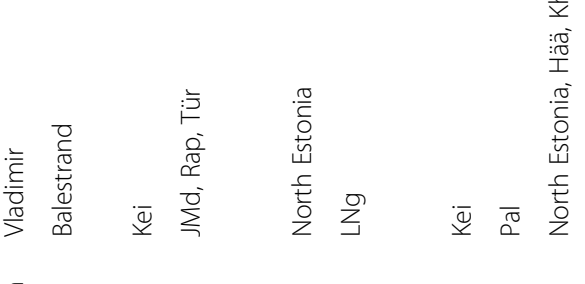
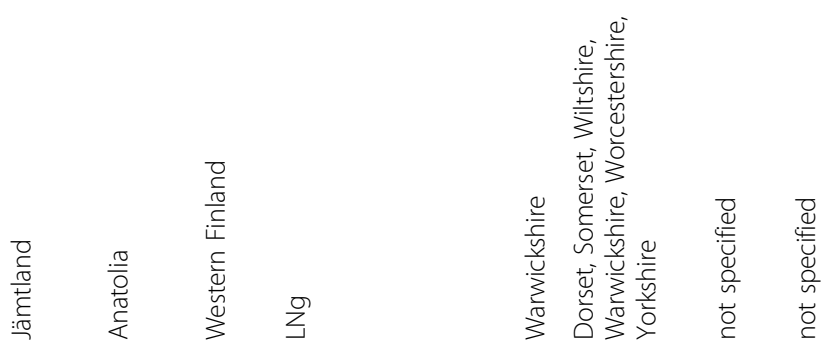

需

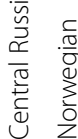

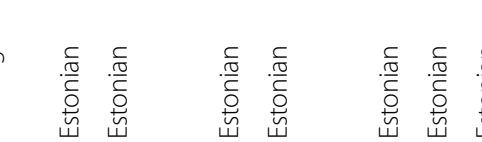

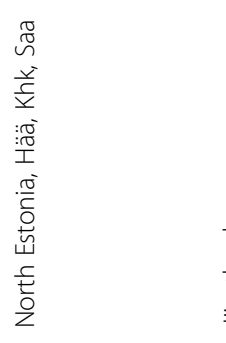

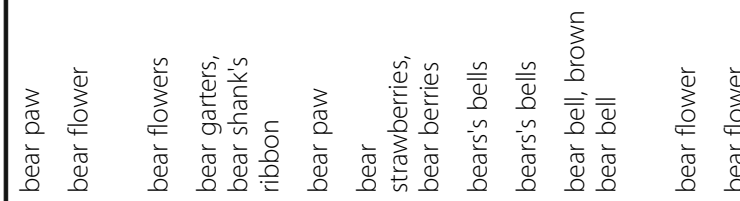

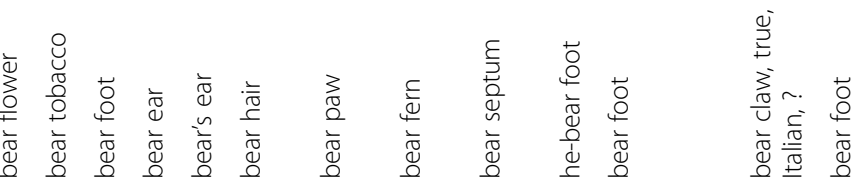

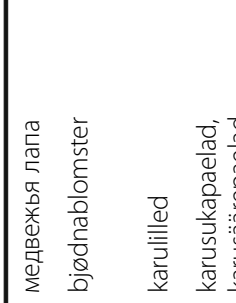
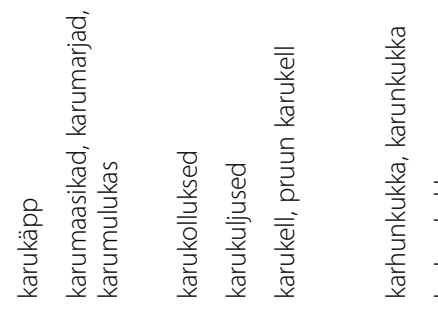

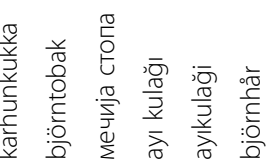

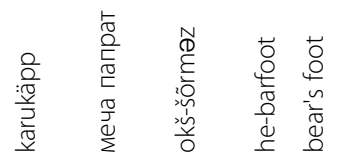
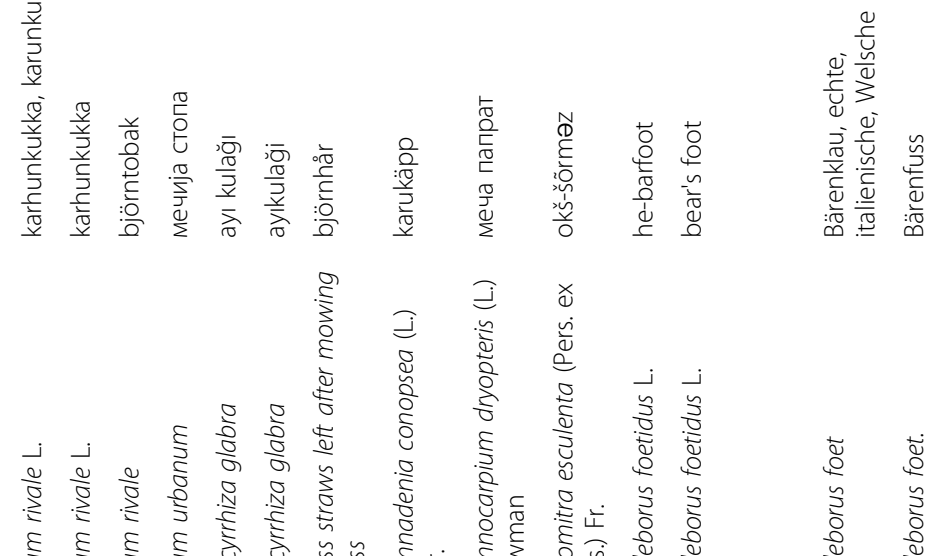

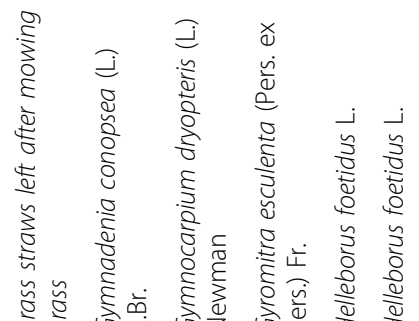

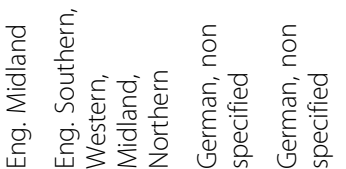




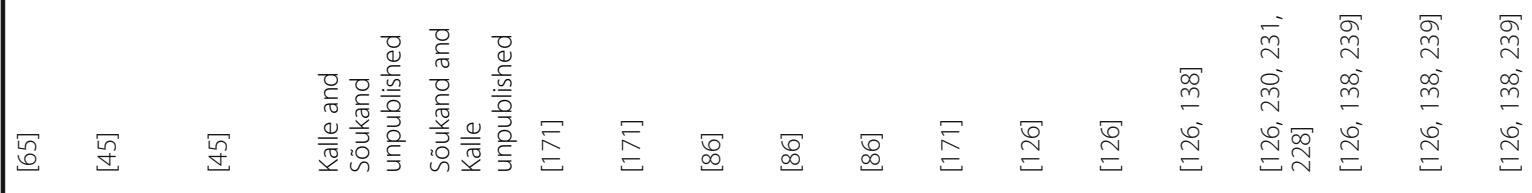

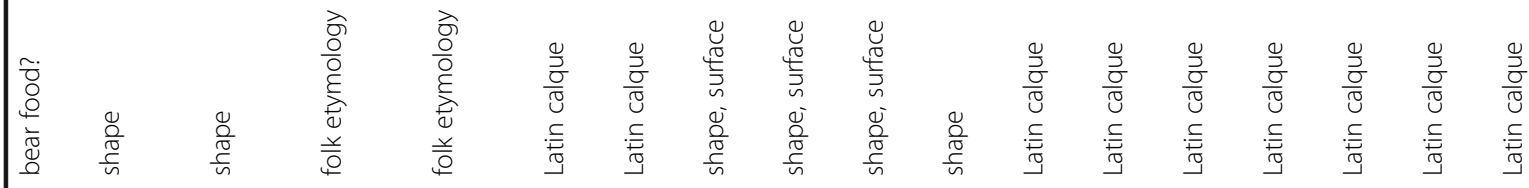

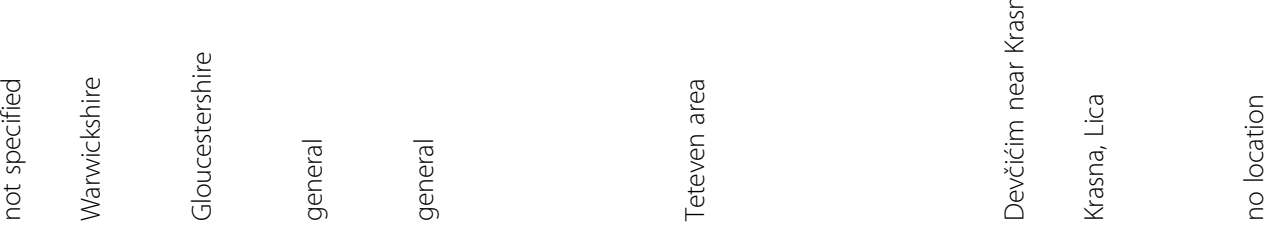

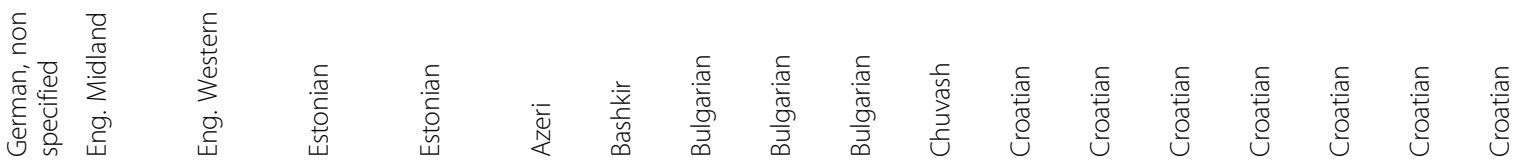

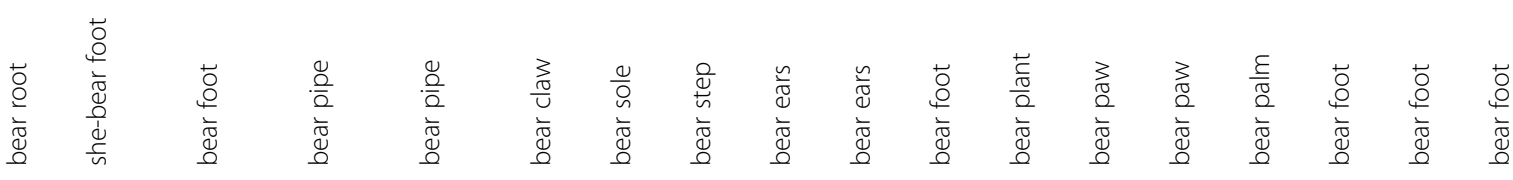

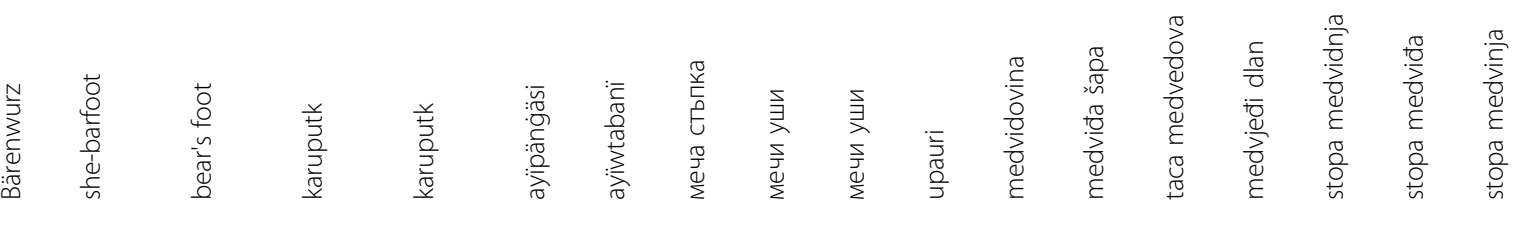

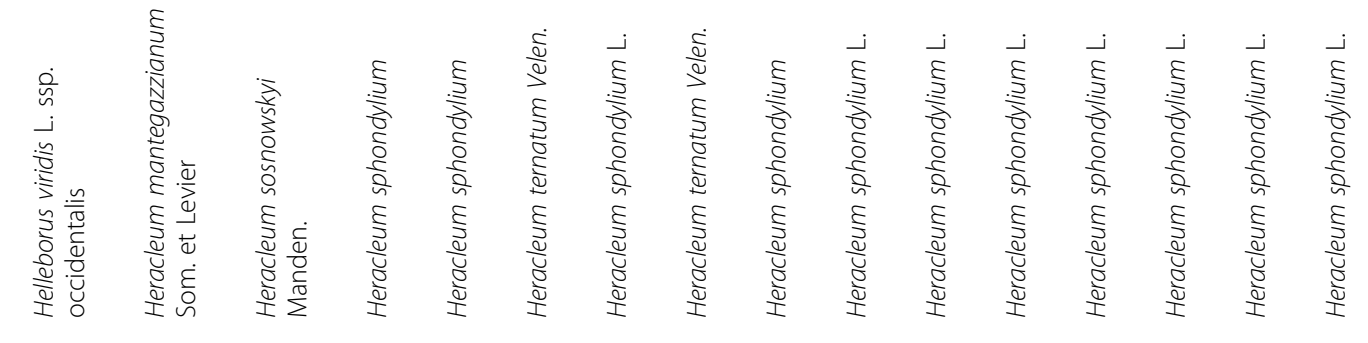
畄 总 


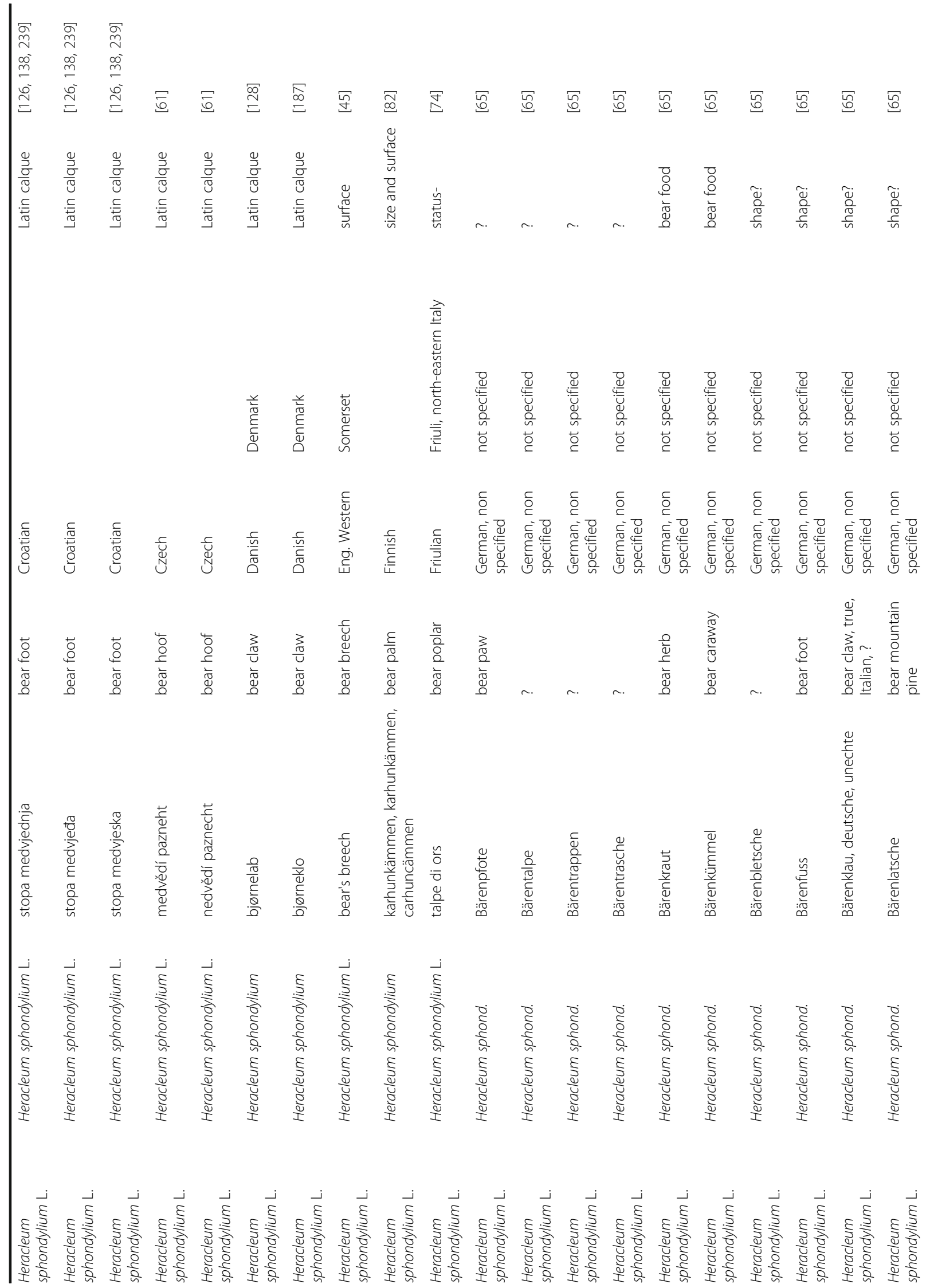




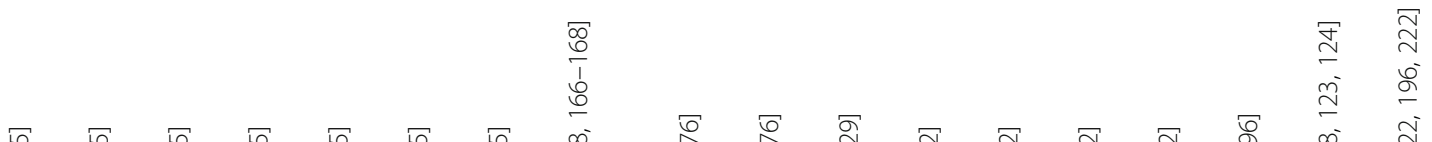

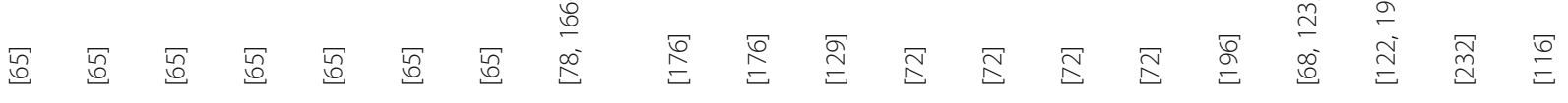

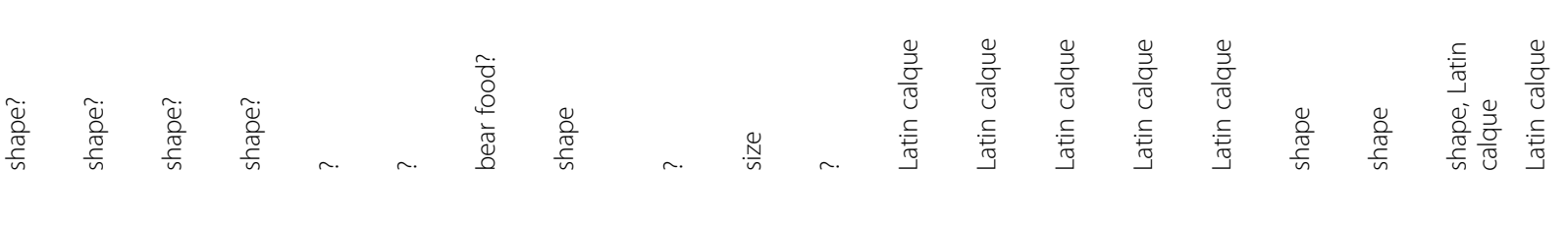

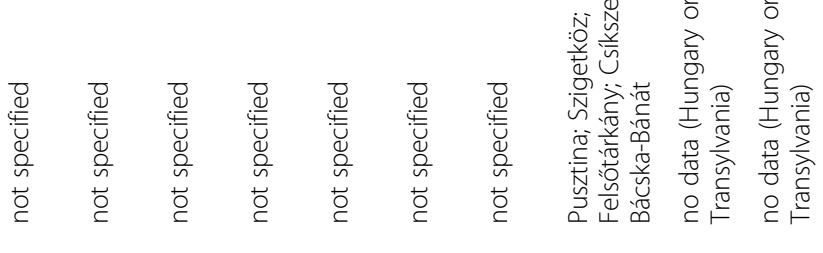

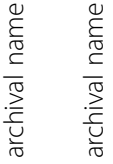

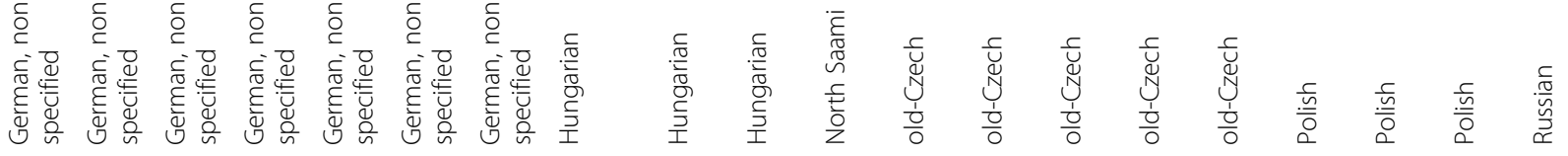

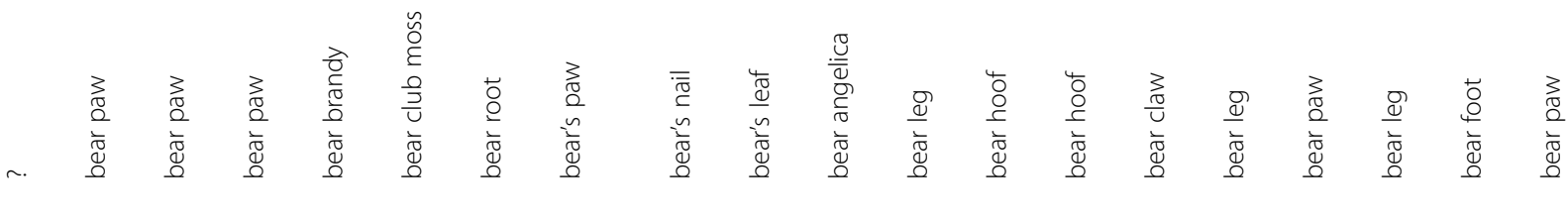

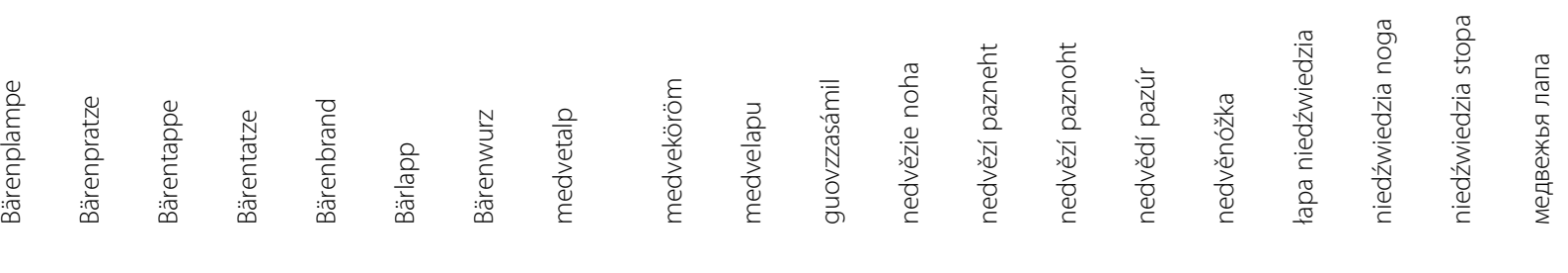

$$
\begin{aligned}
& \frac{2}{\frac{1}{3}}
\end{aligned}
$$

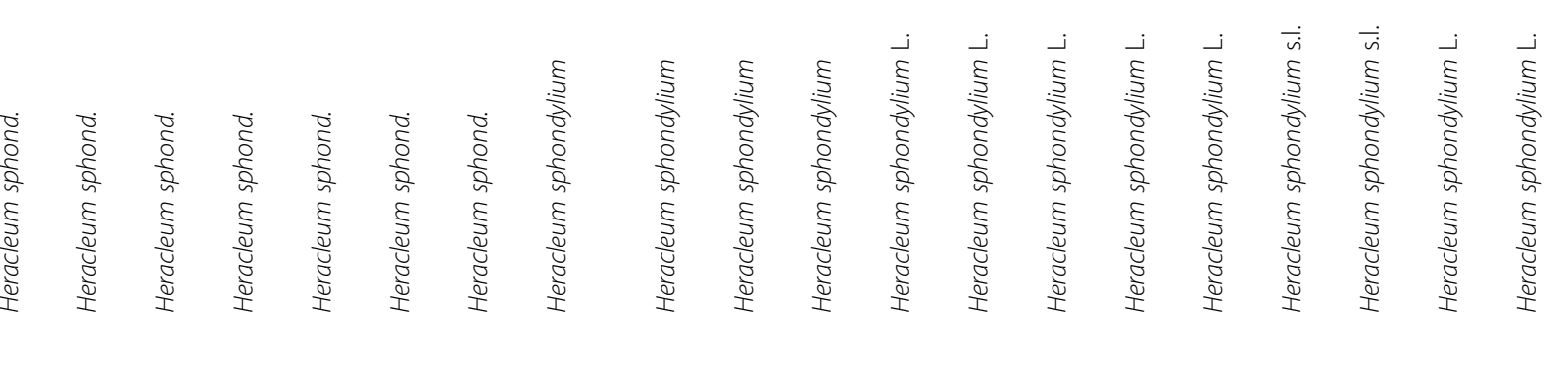

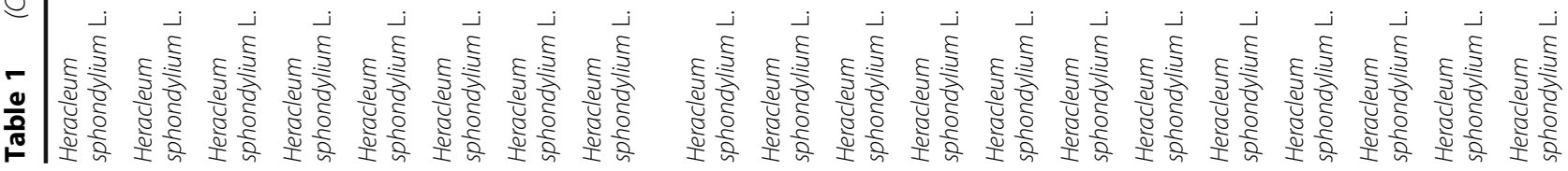




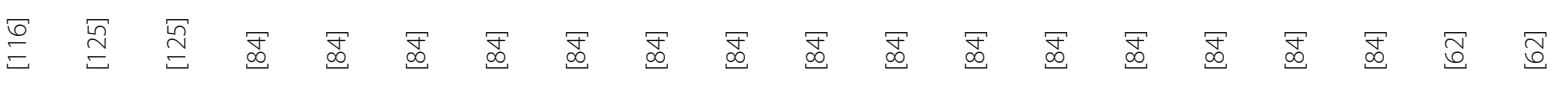

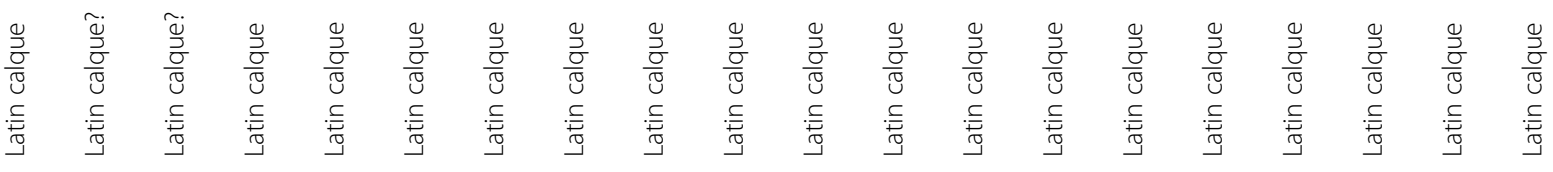

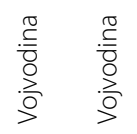

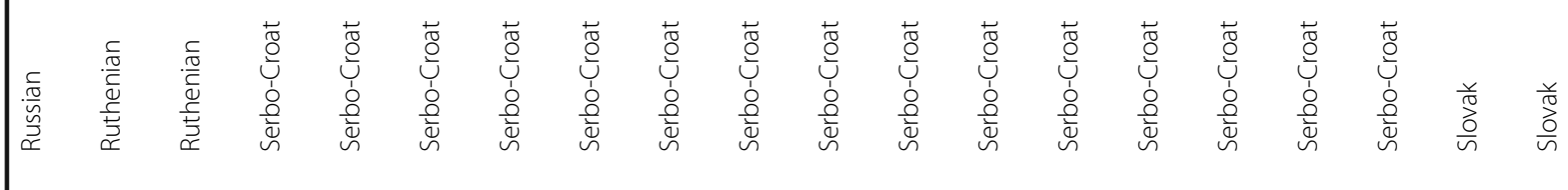

$\stackrel{\frac{0}{\pi}}{\frac{\pi}{\pi}}$

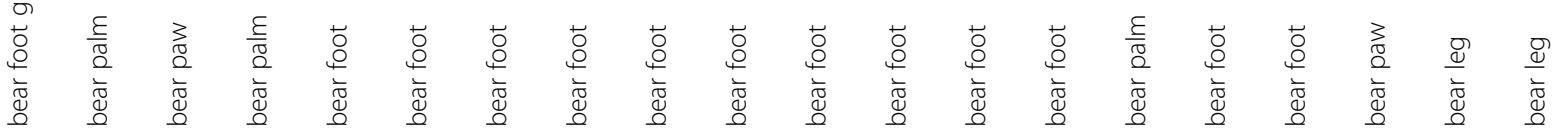




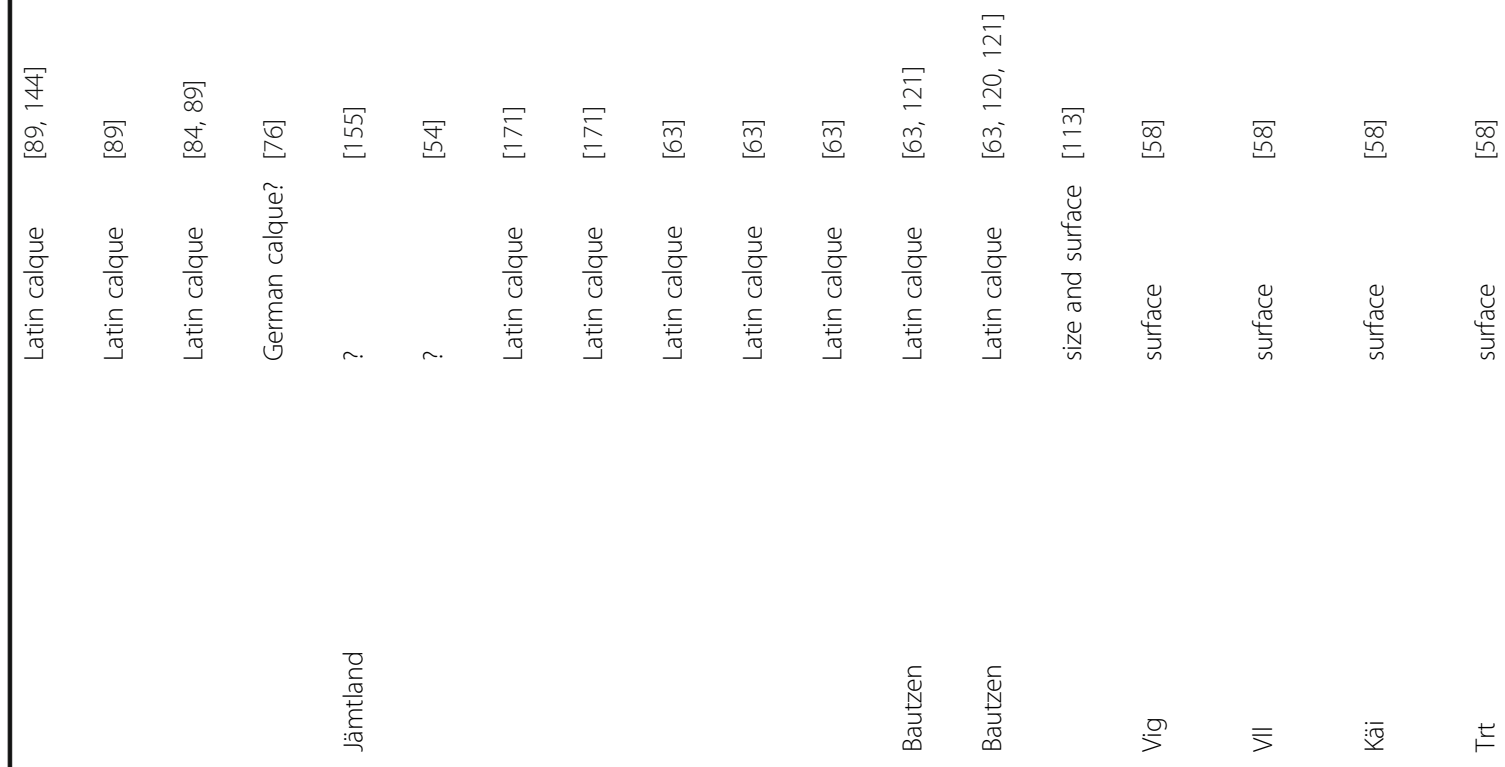

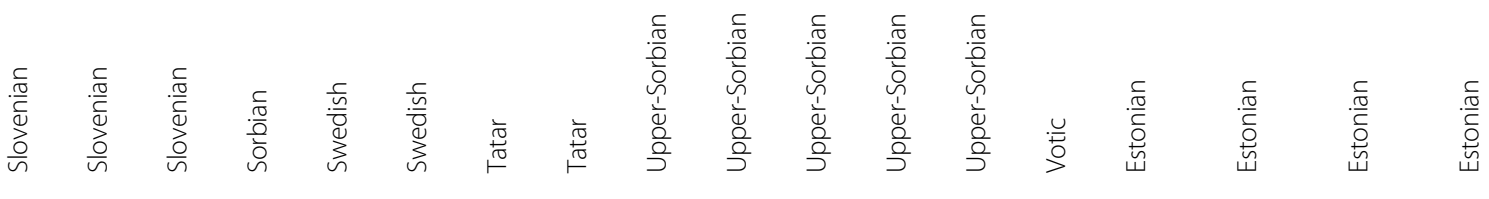

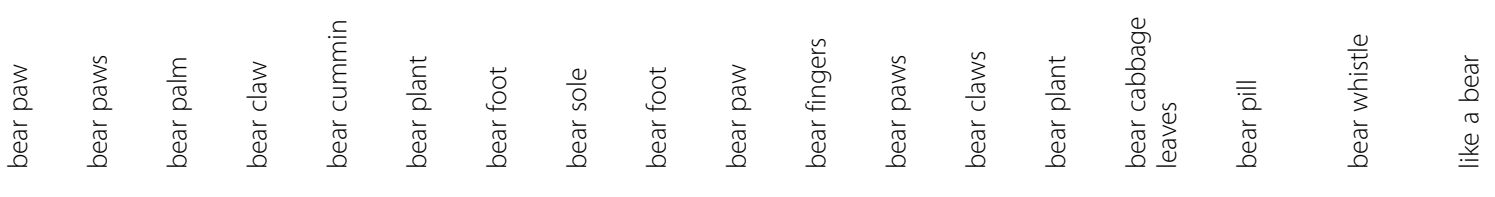

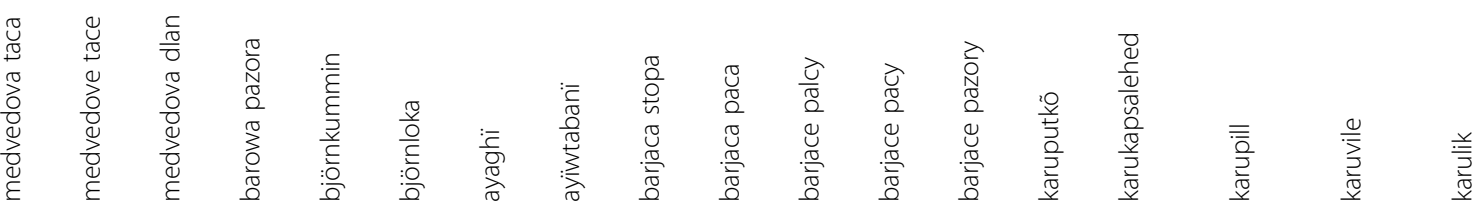

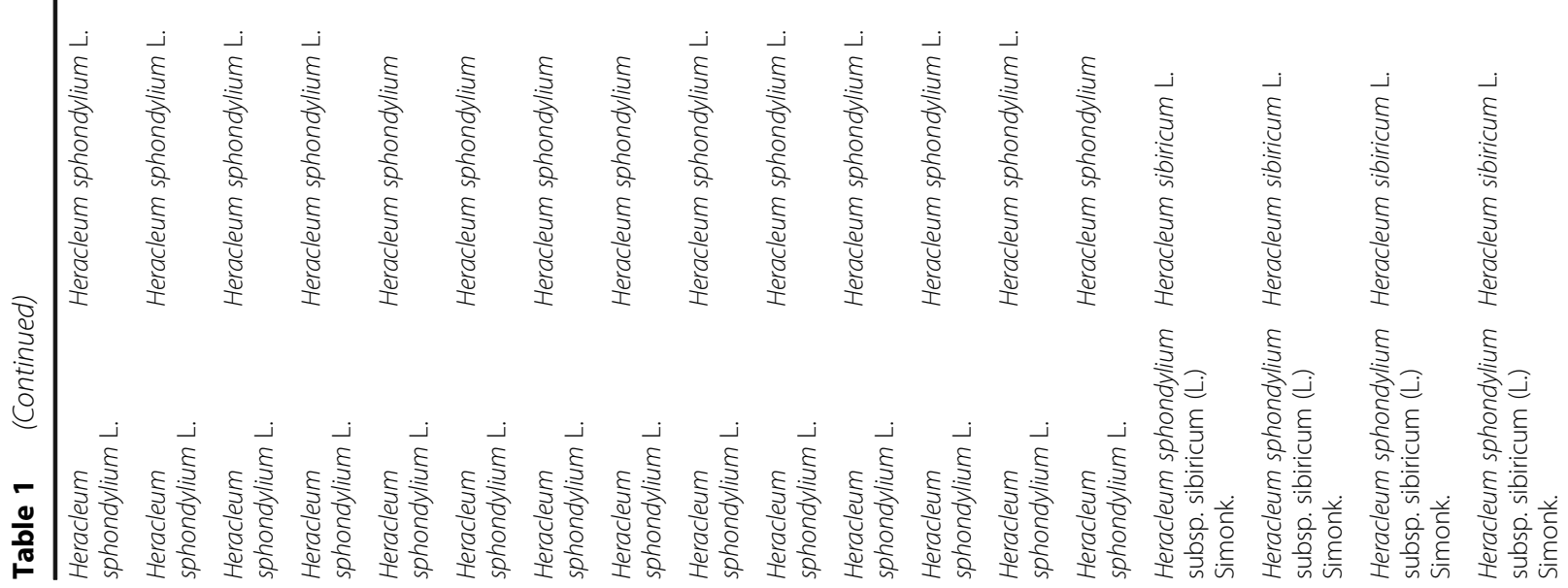




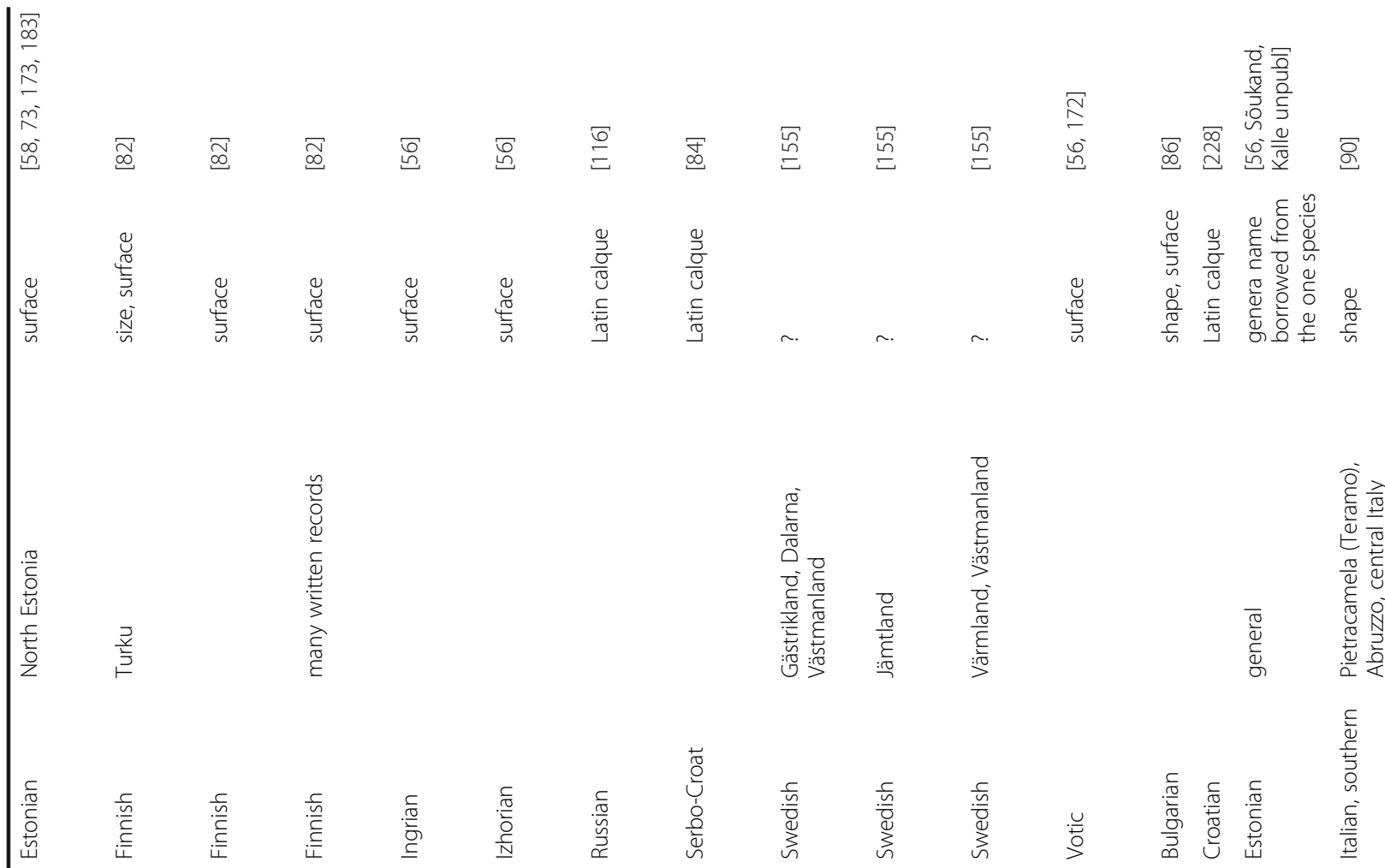

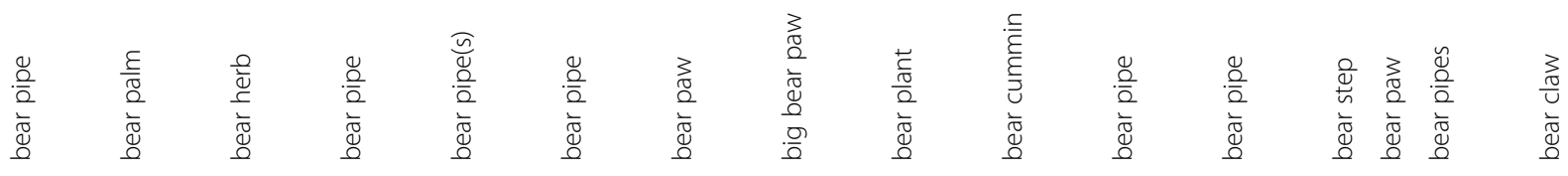

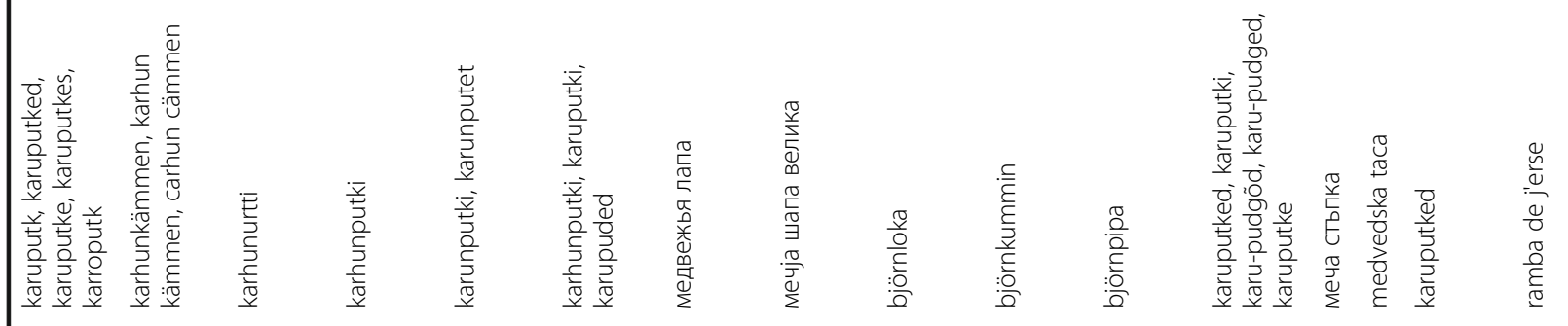

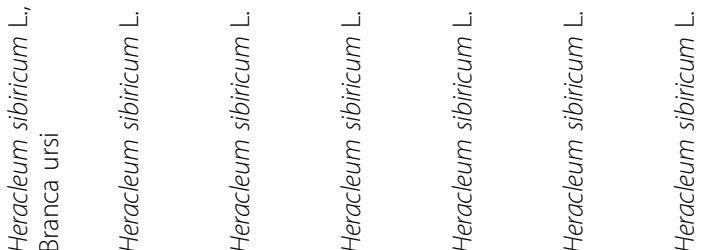

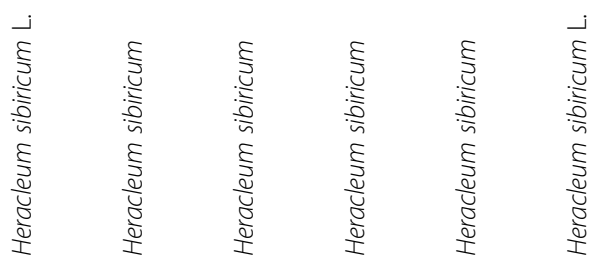

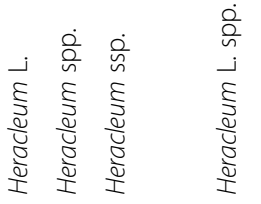




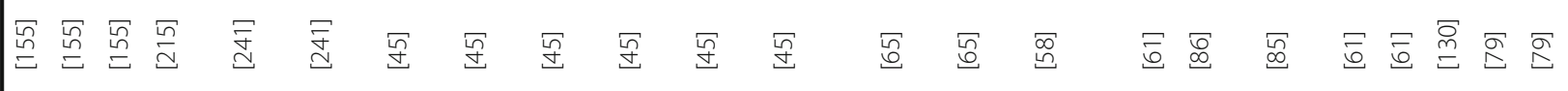

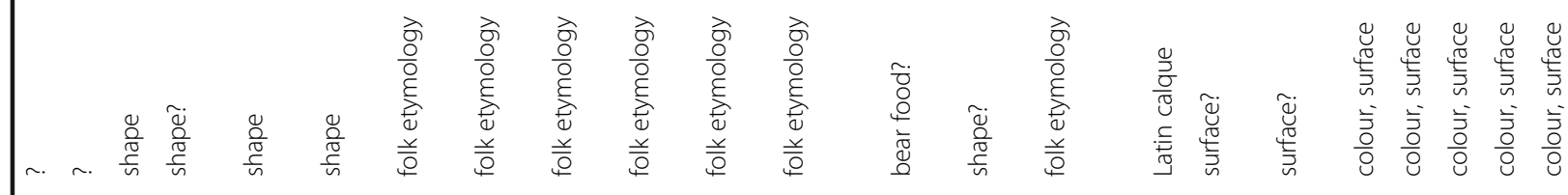

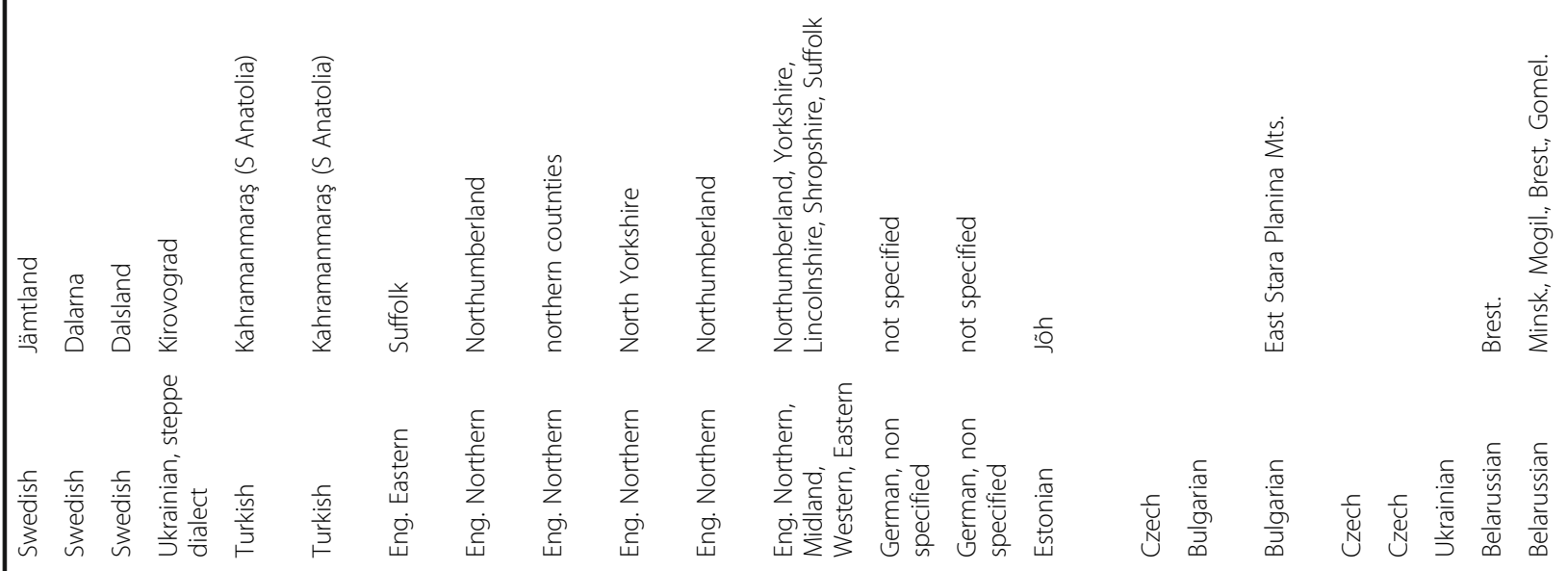

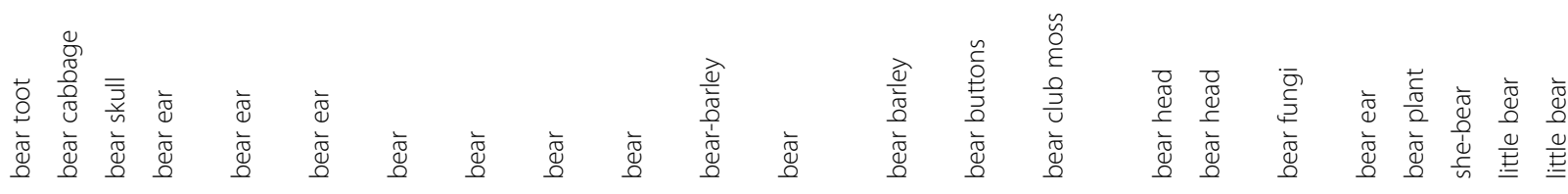

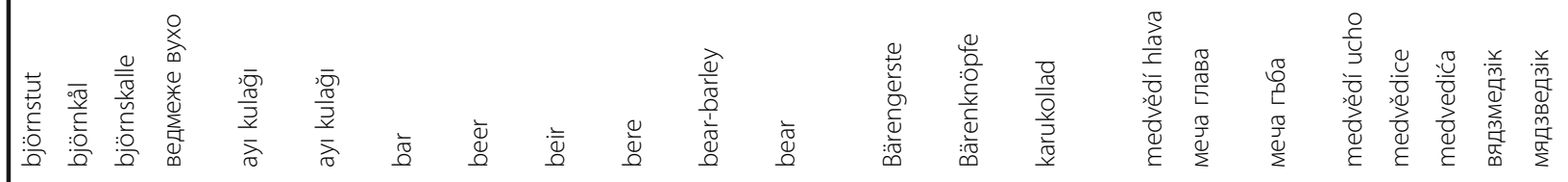

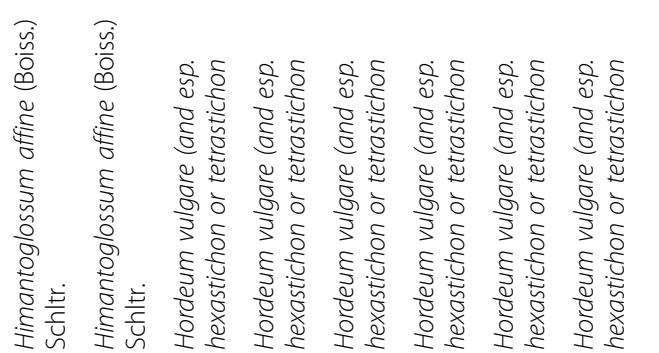

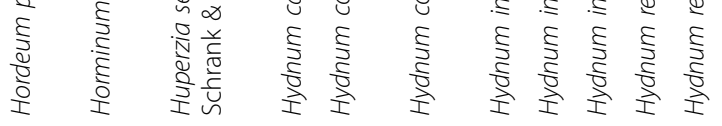

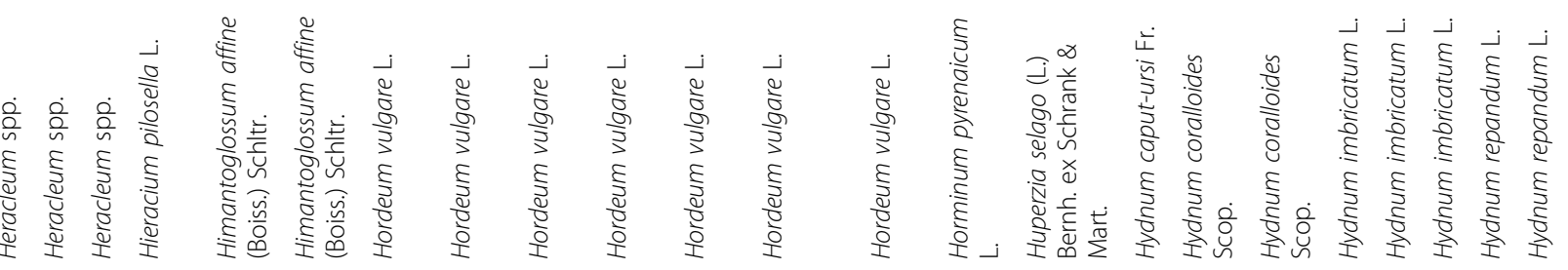




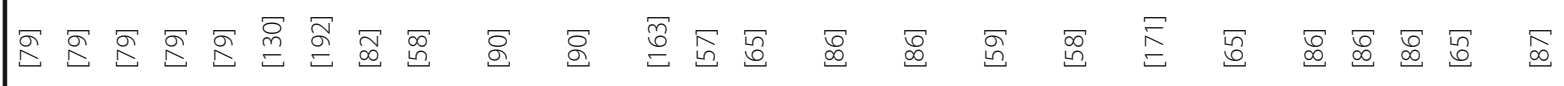

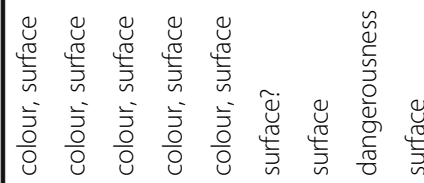

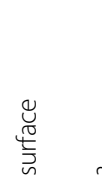

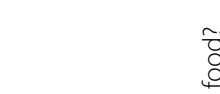

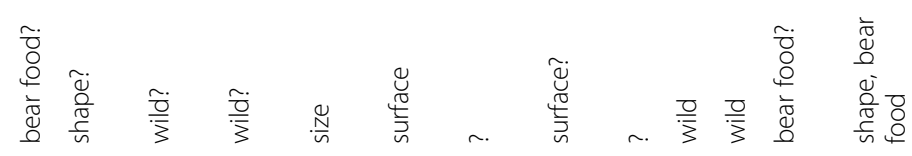

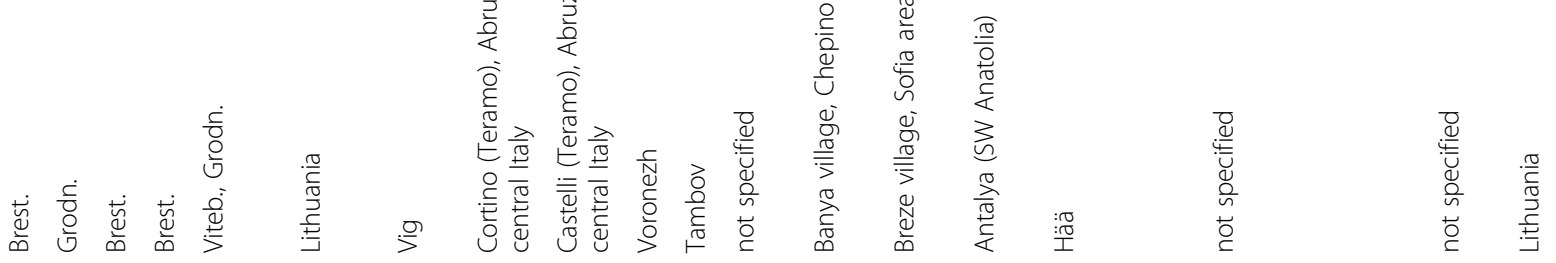

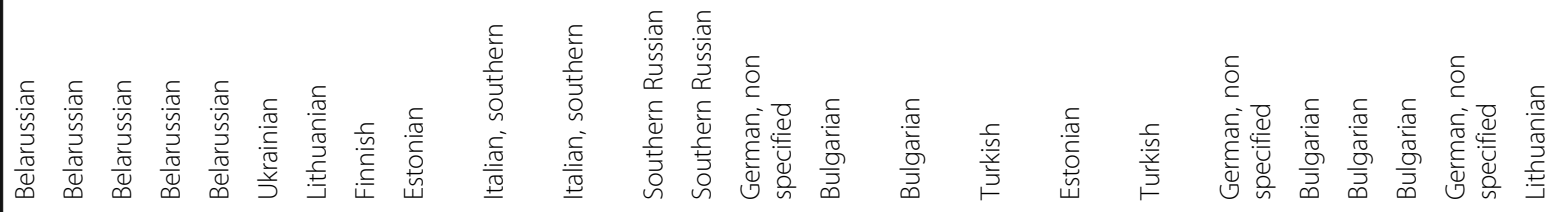

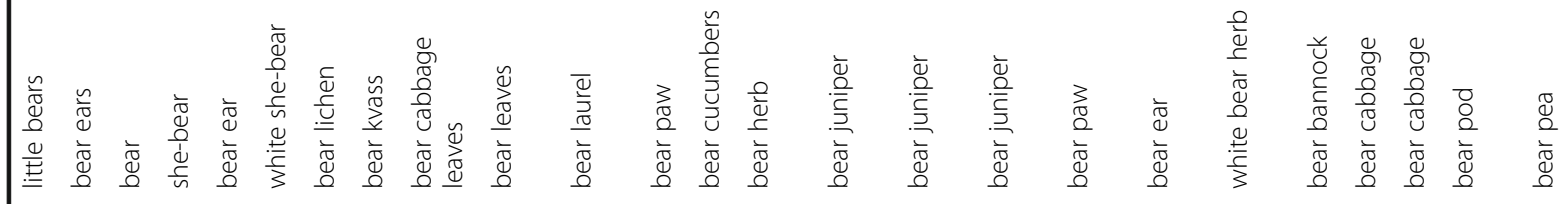

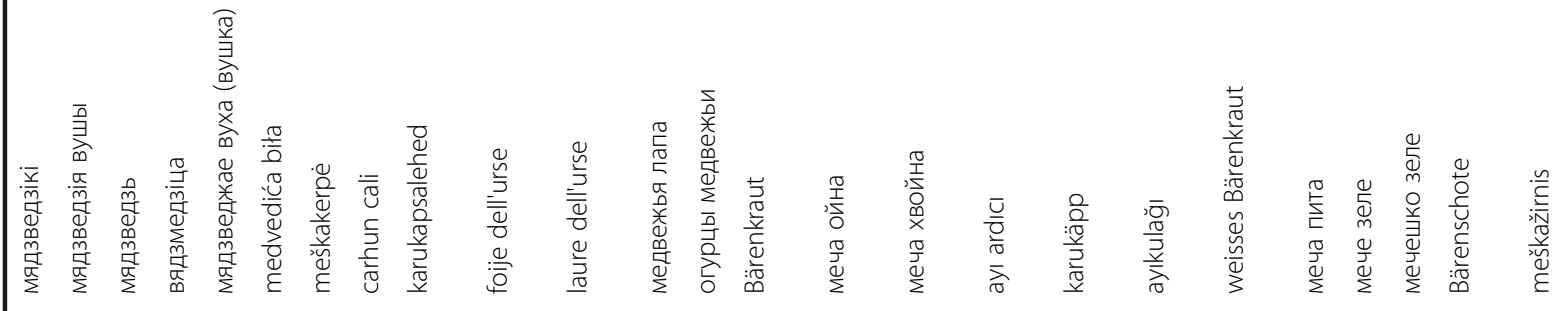




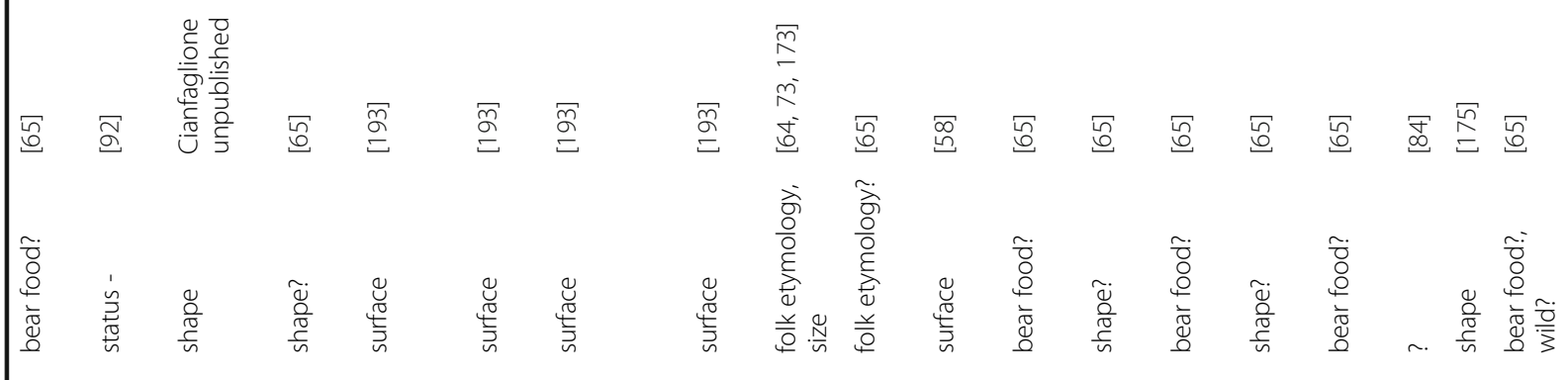

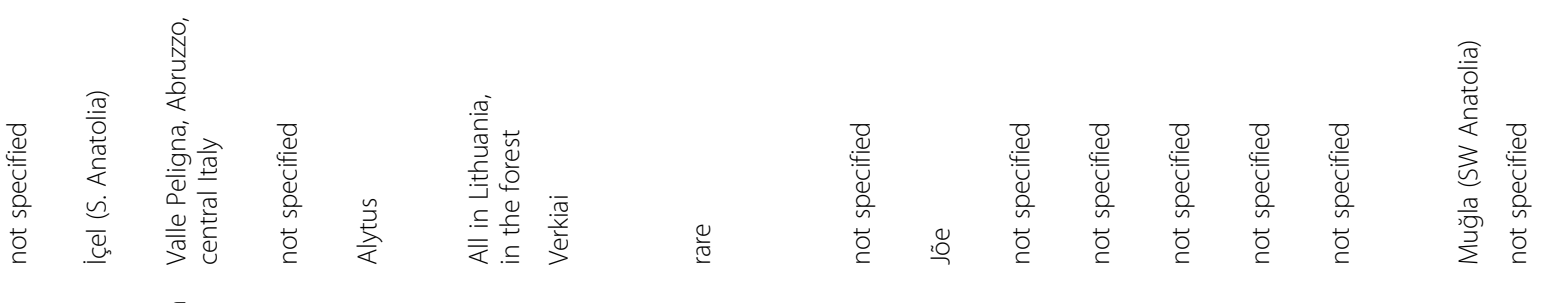

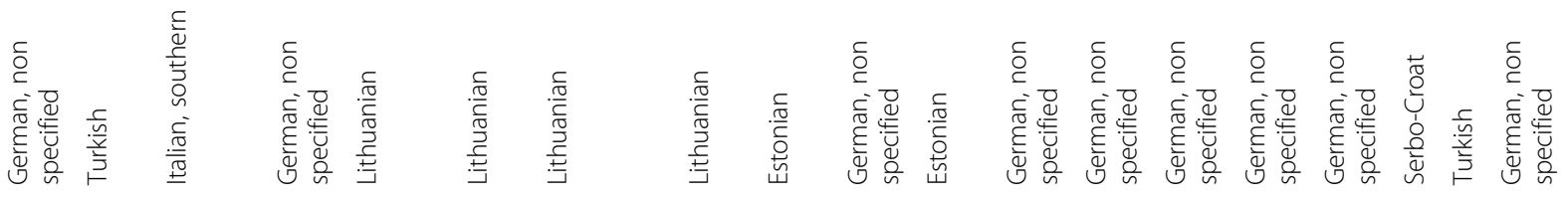

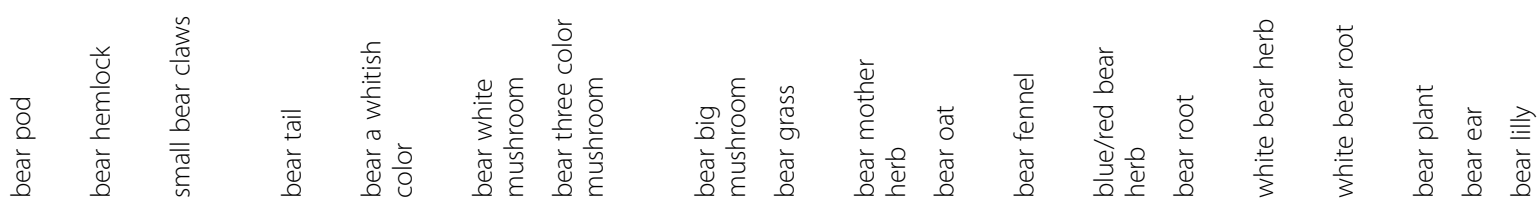

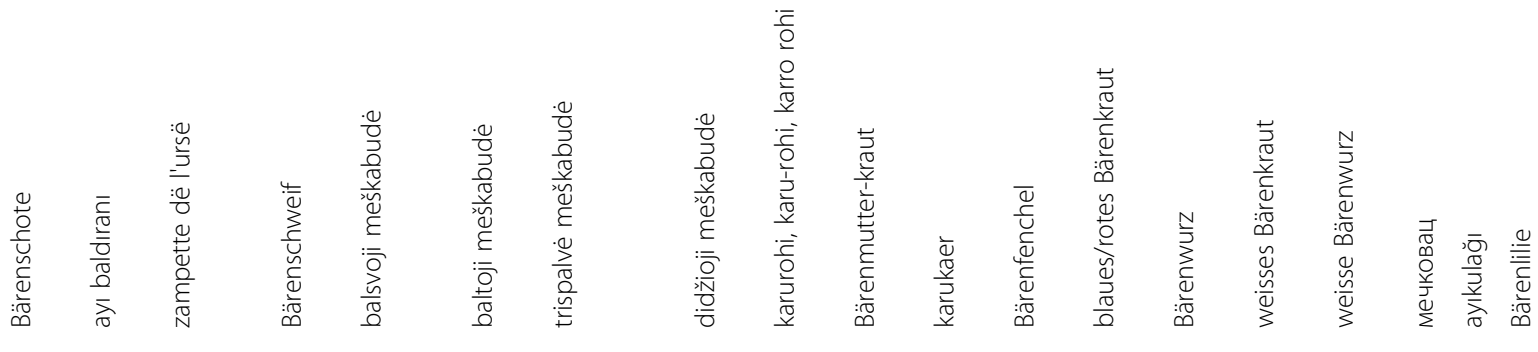

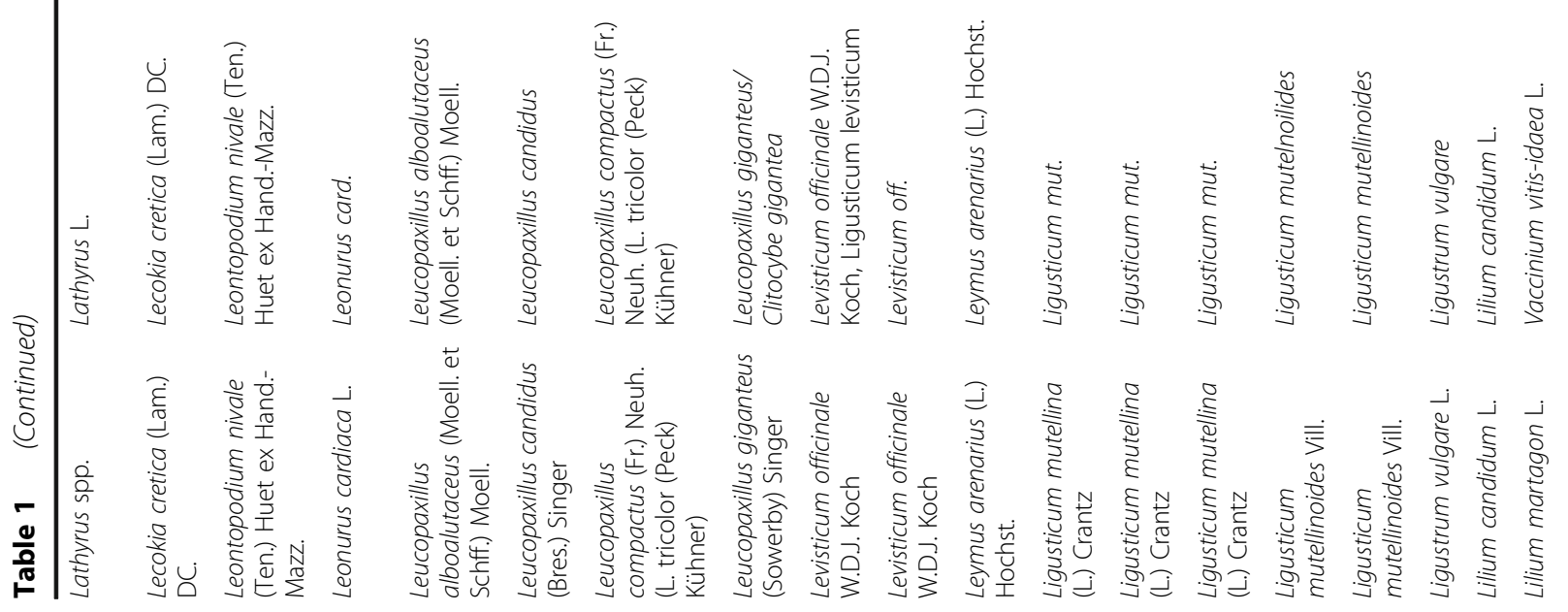




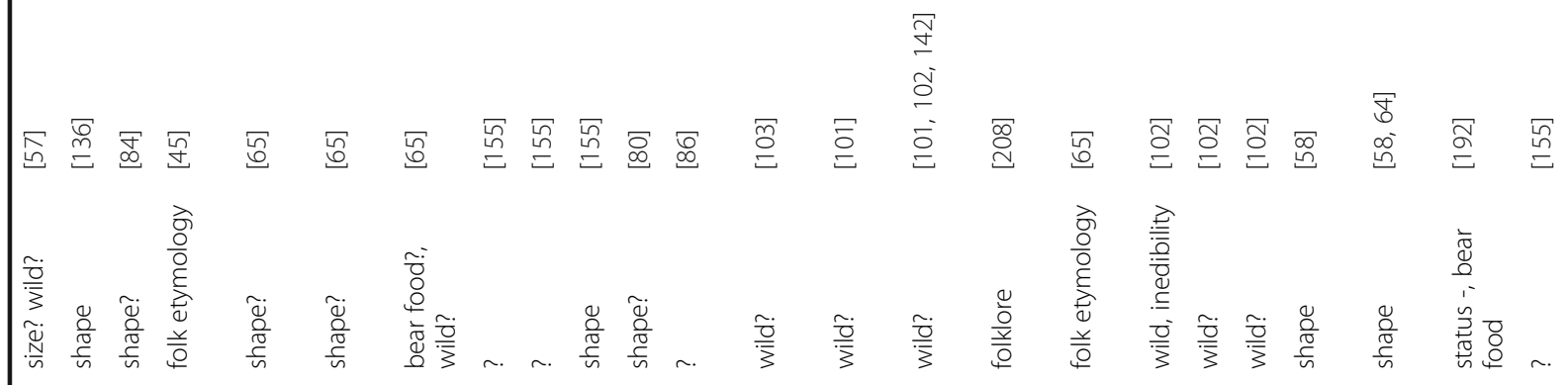

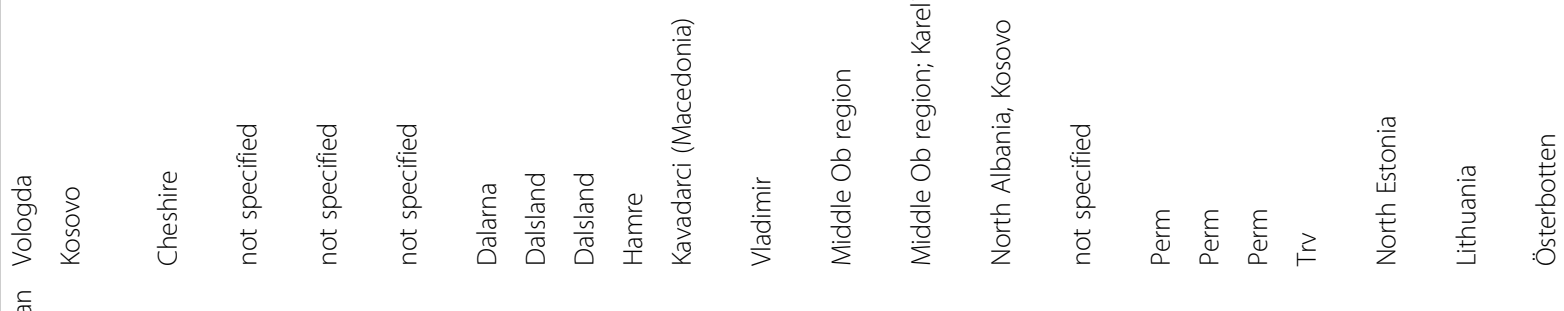

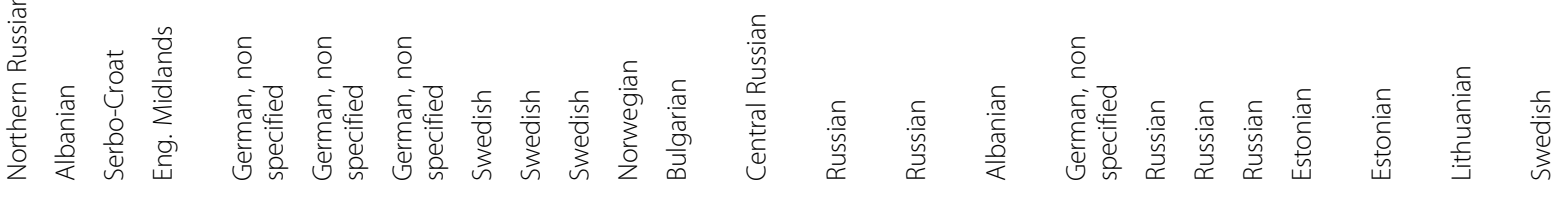

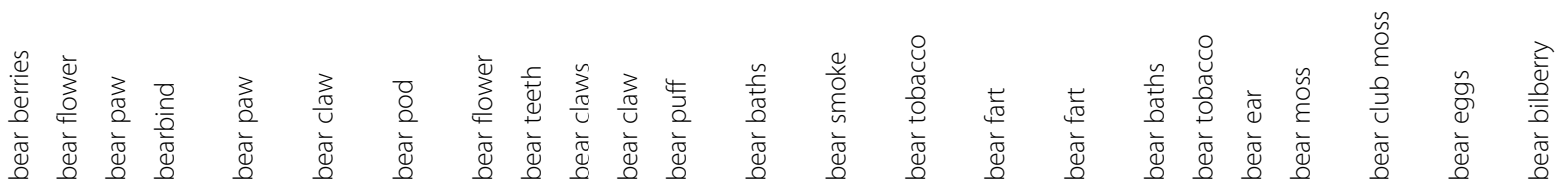

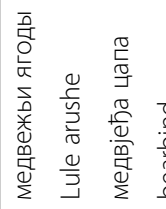

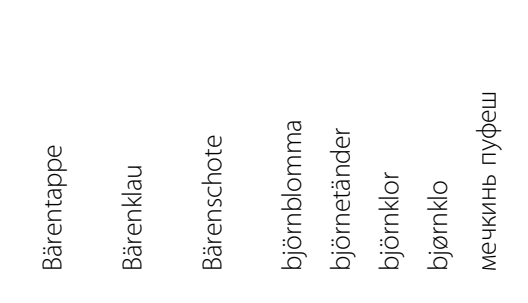

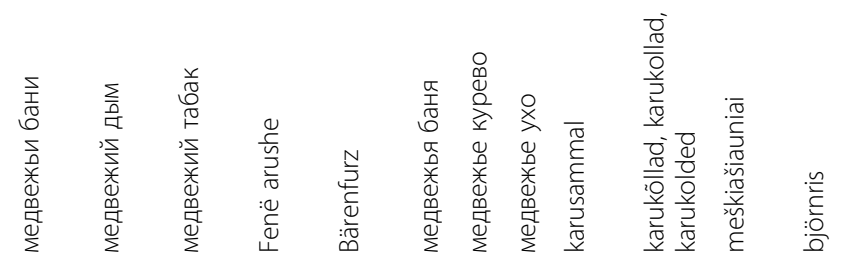

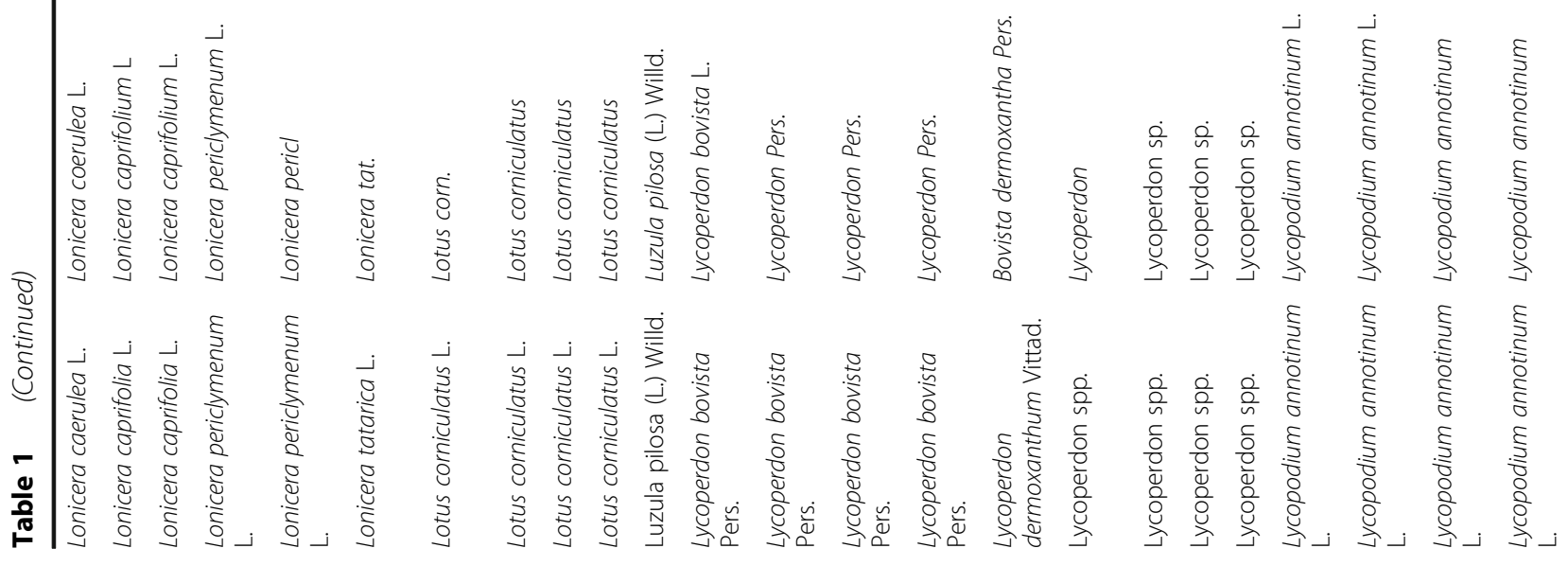




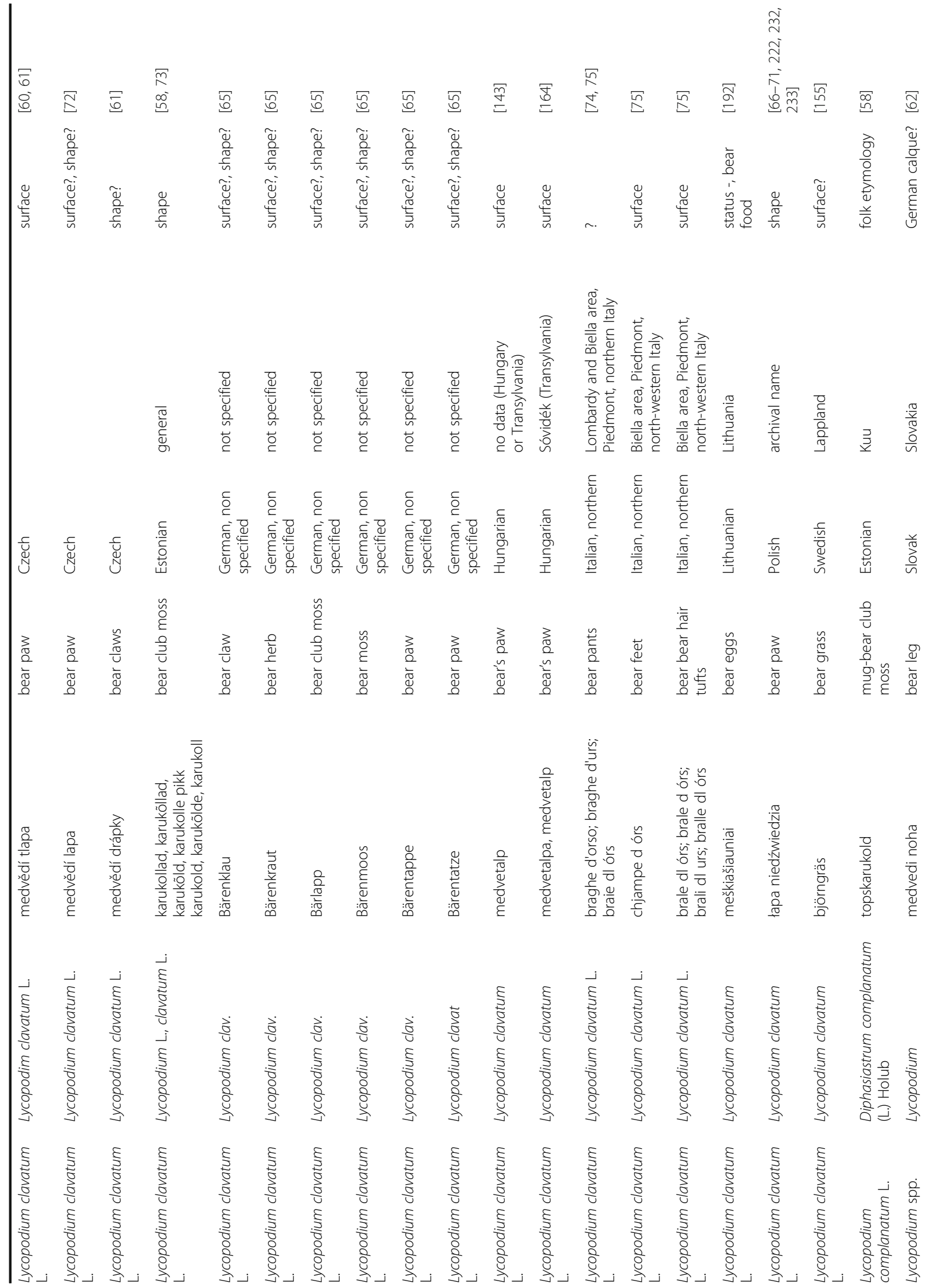




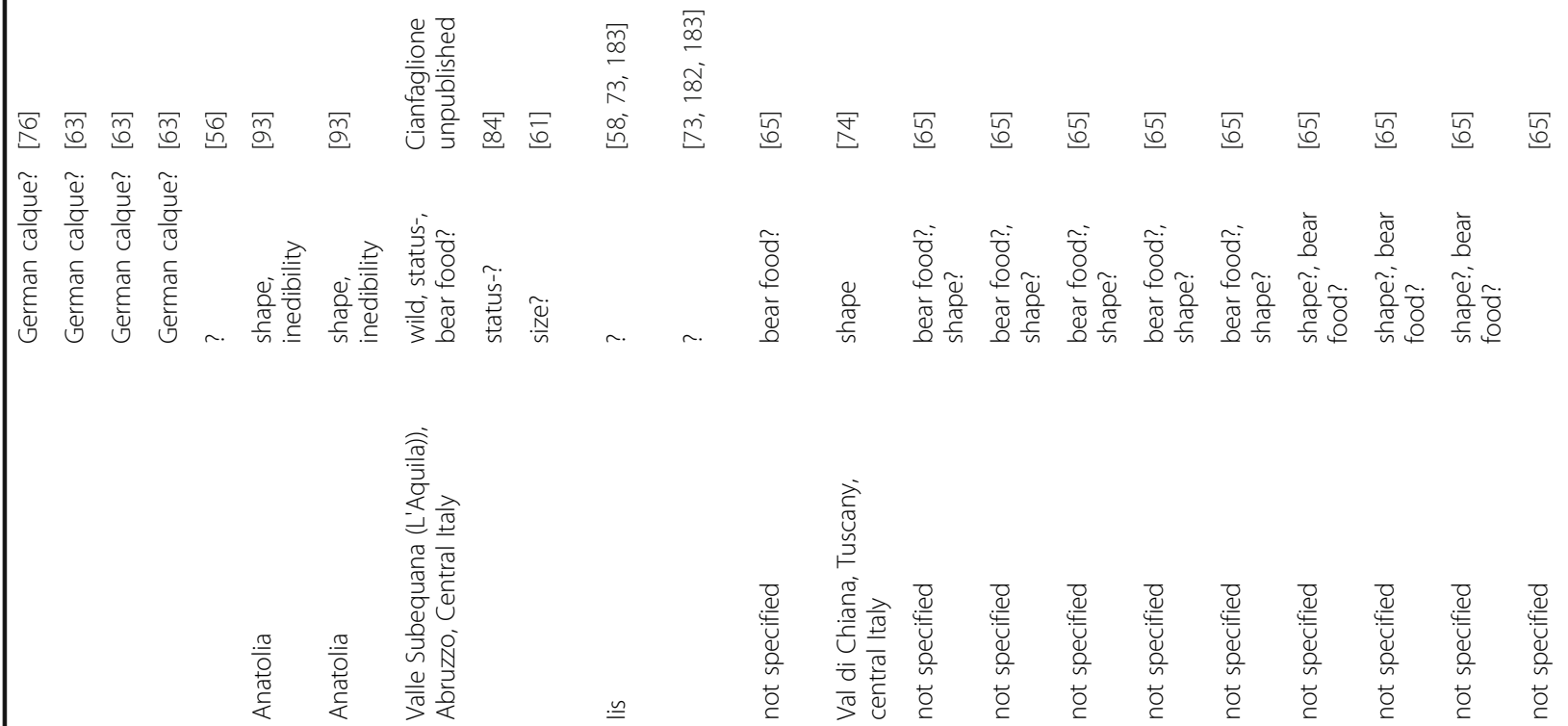

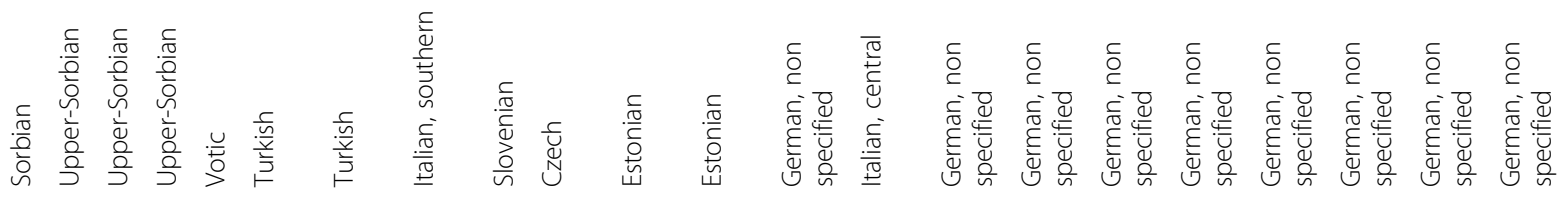

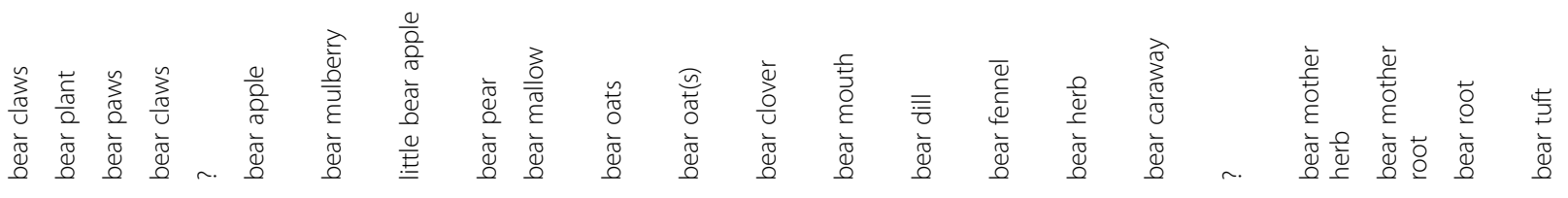

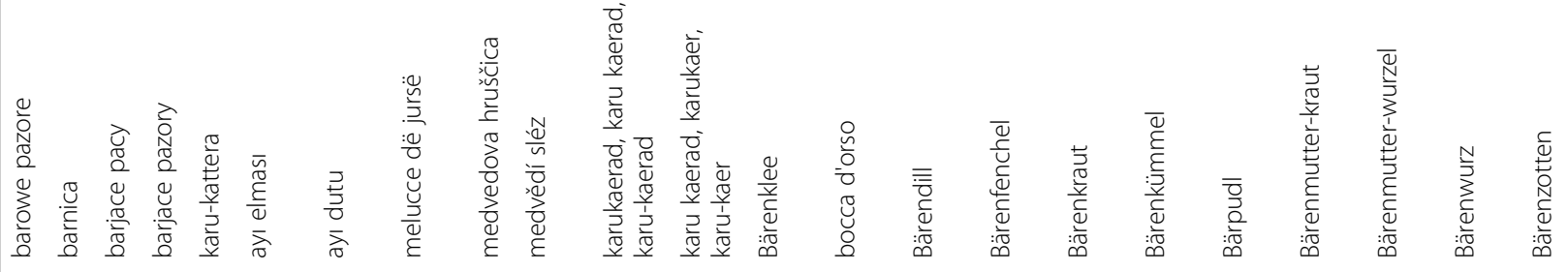




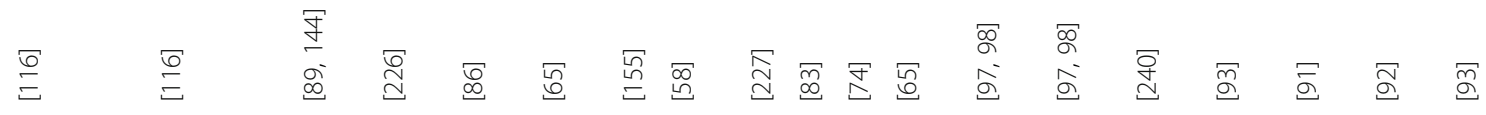

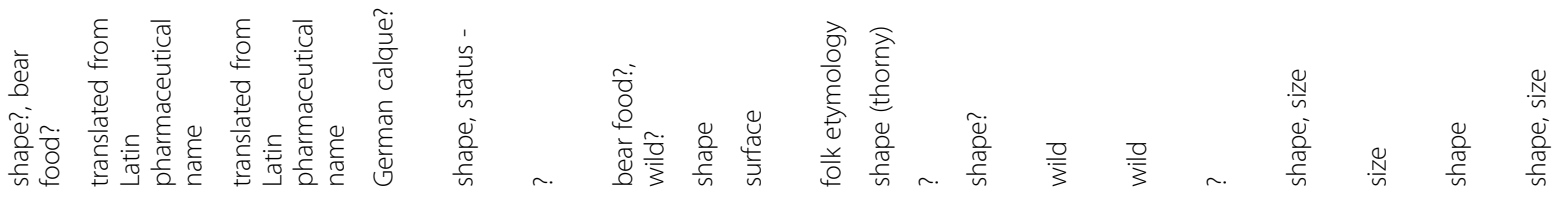

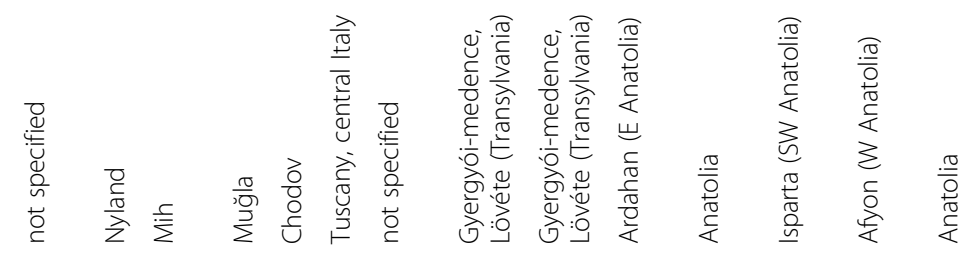

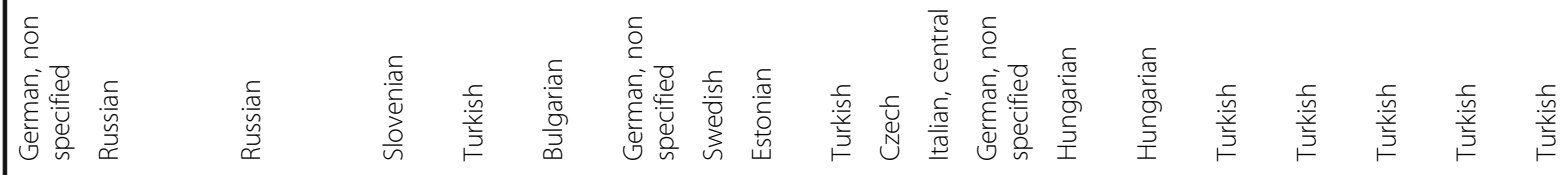

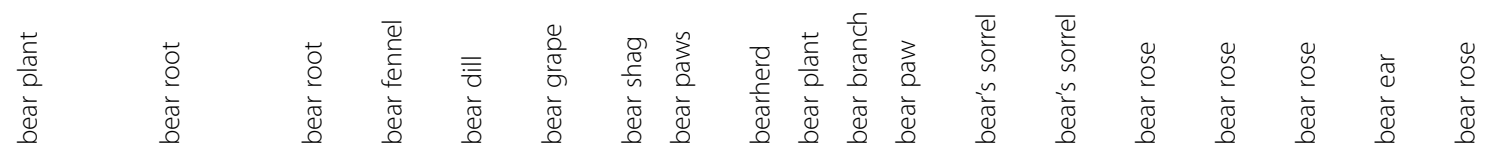

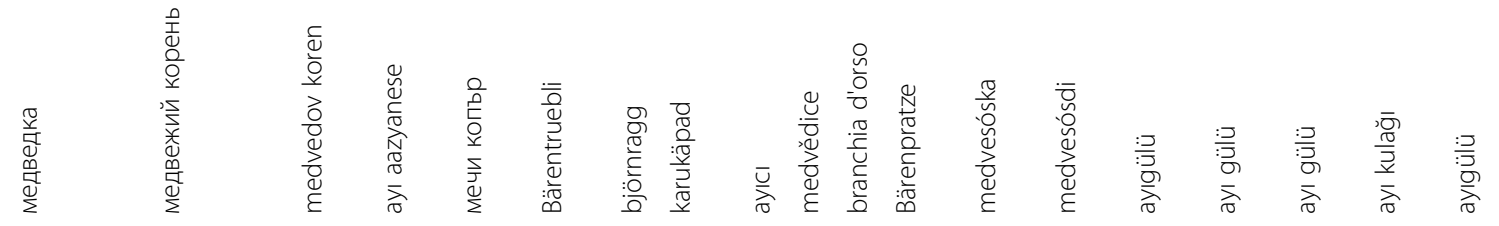

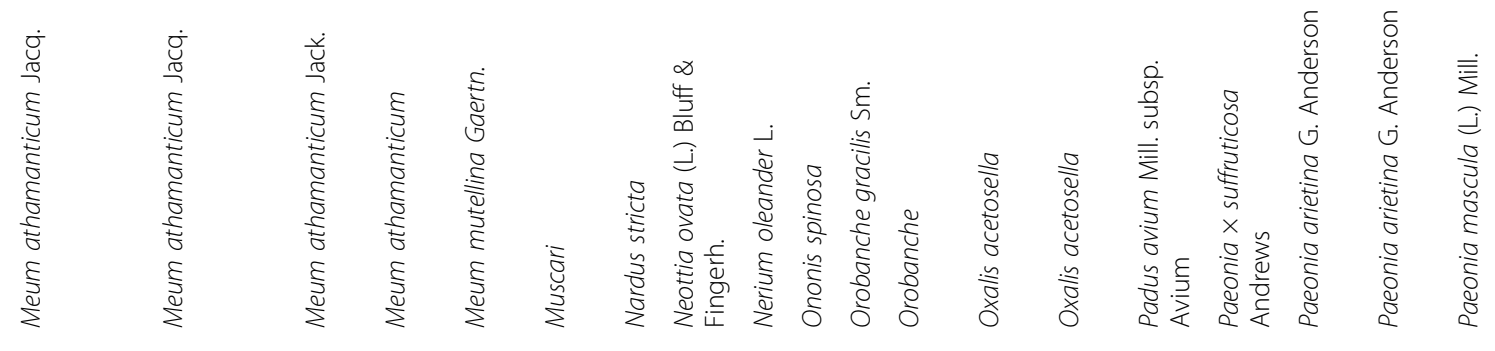

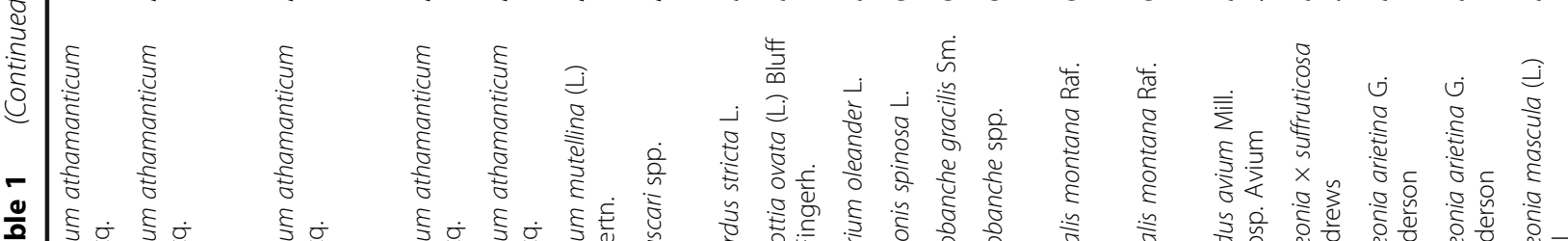

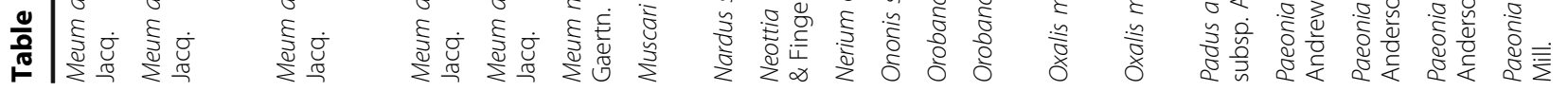




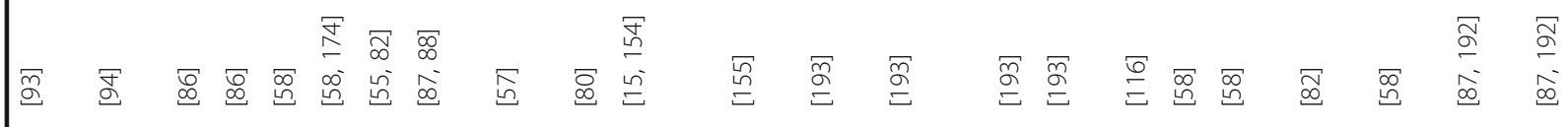

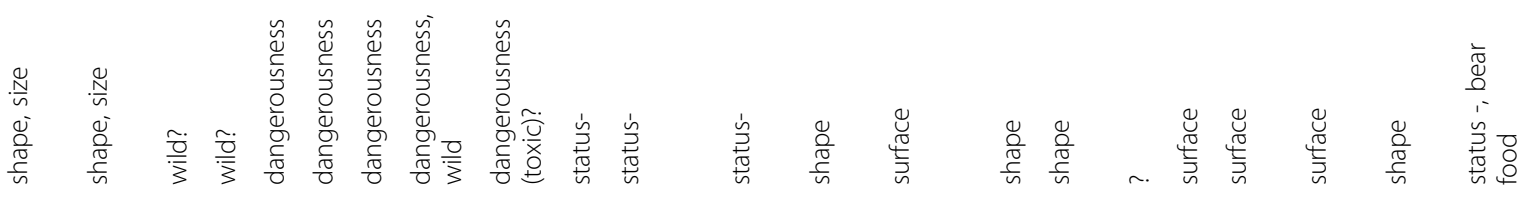

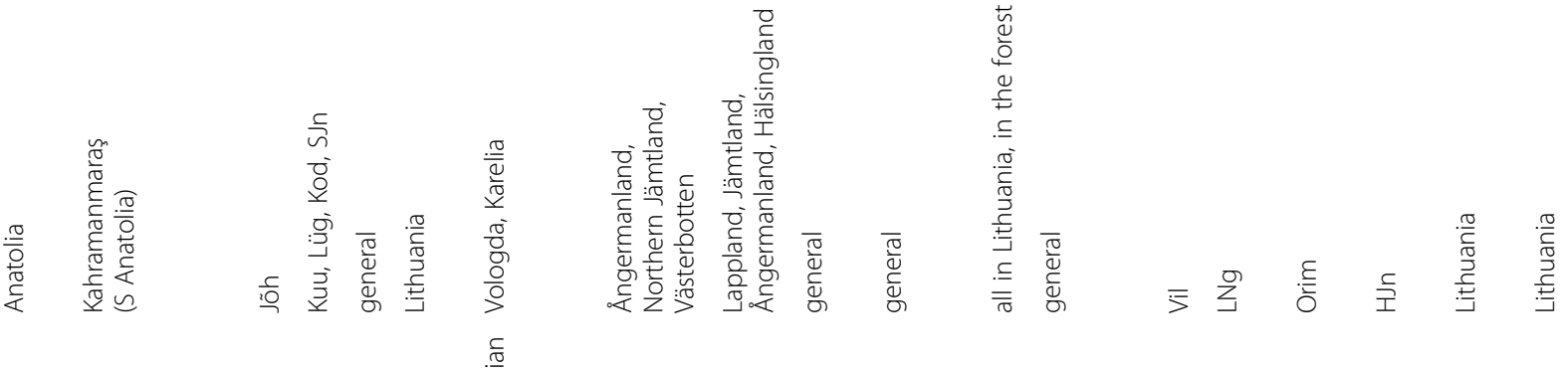

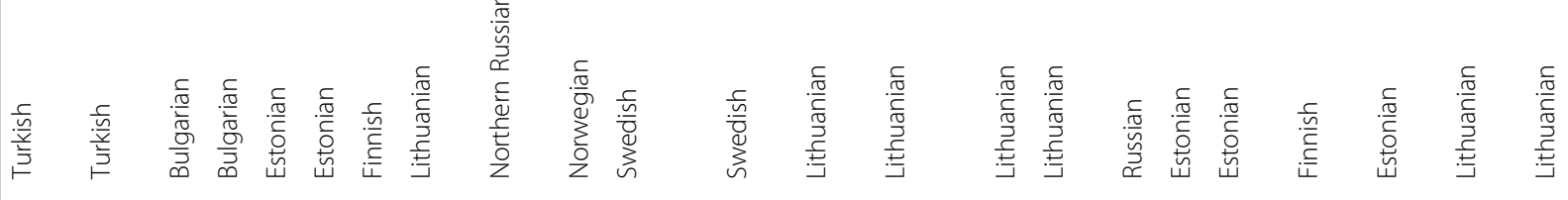

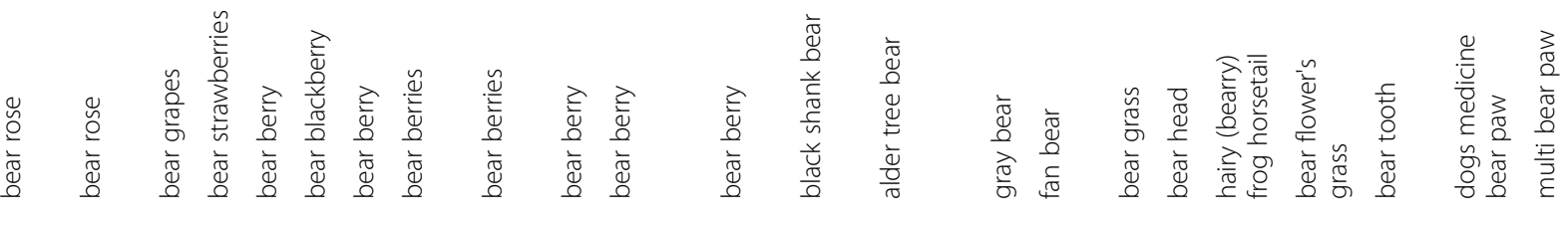

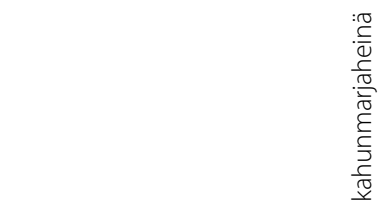

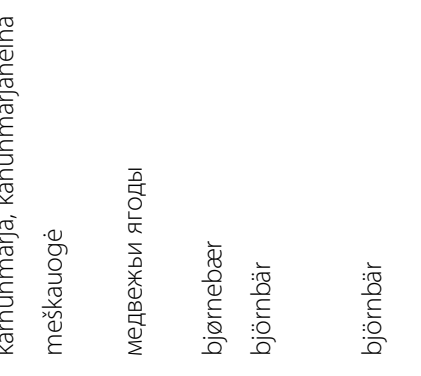

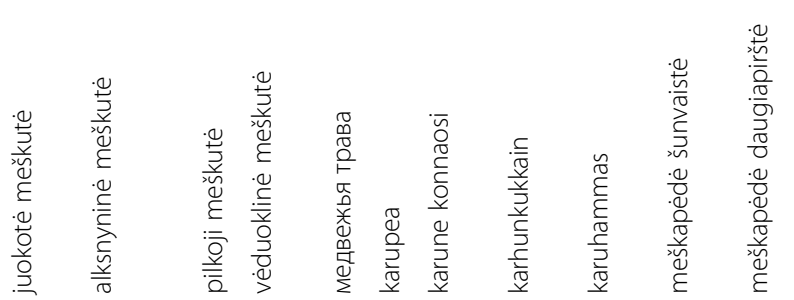

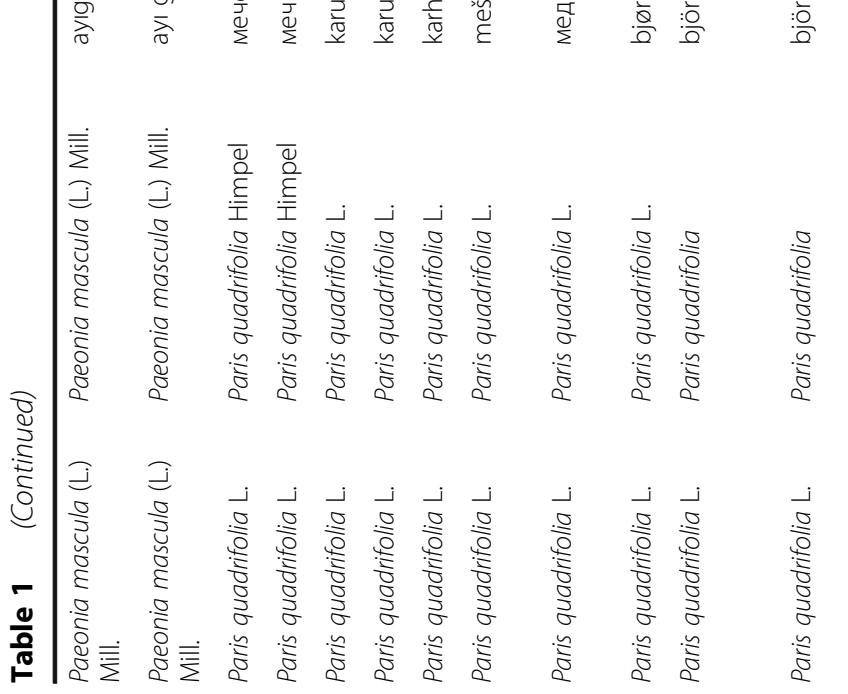
竞

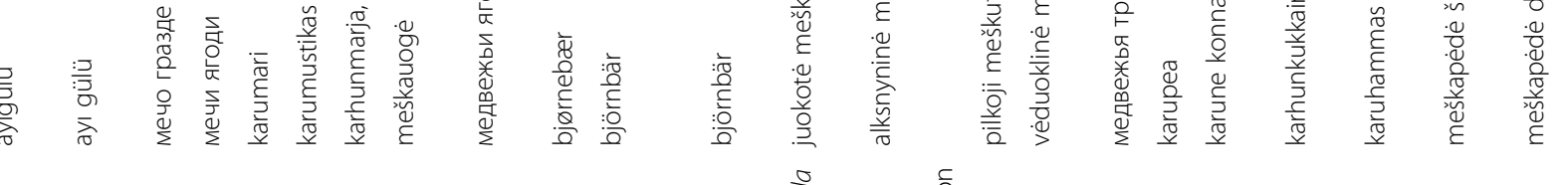
告

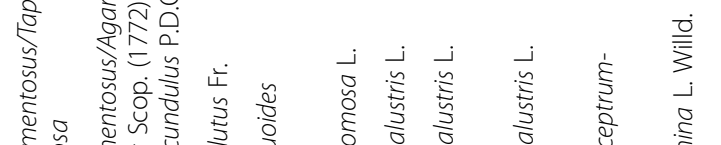

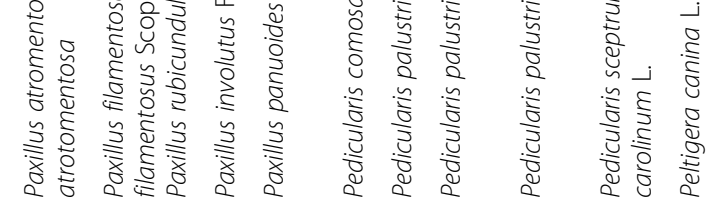

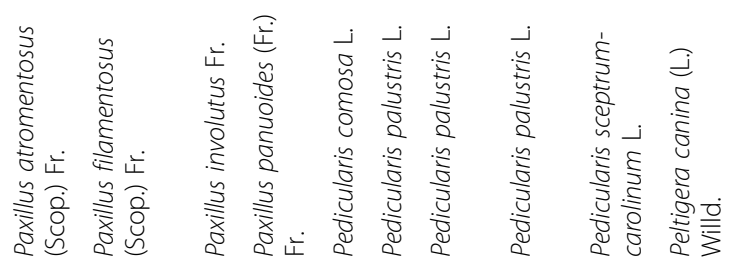




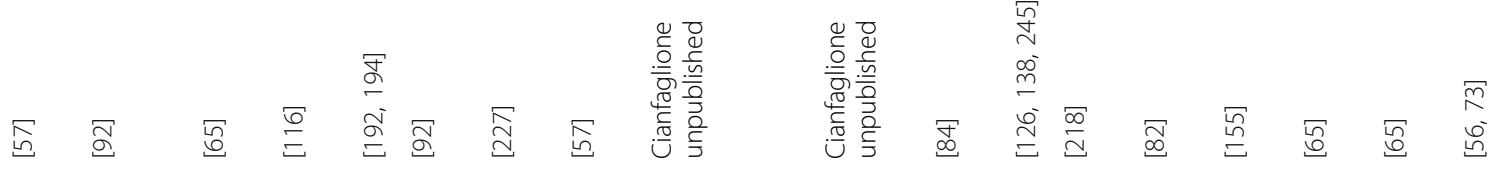

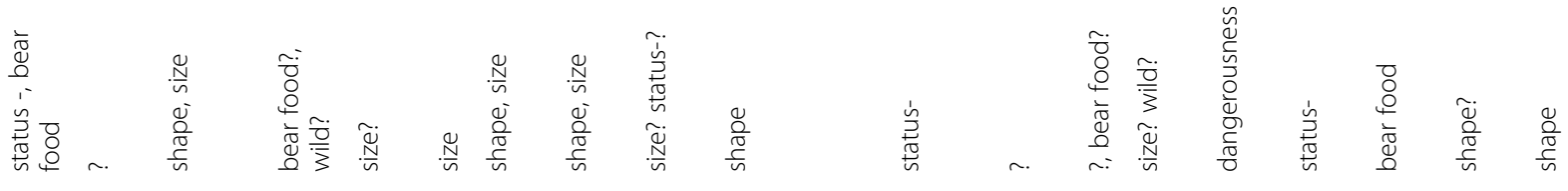

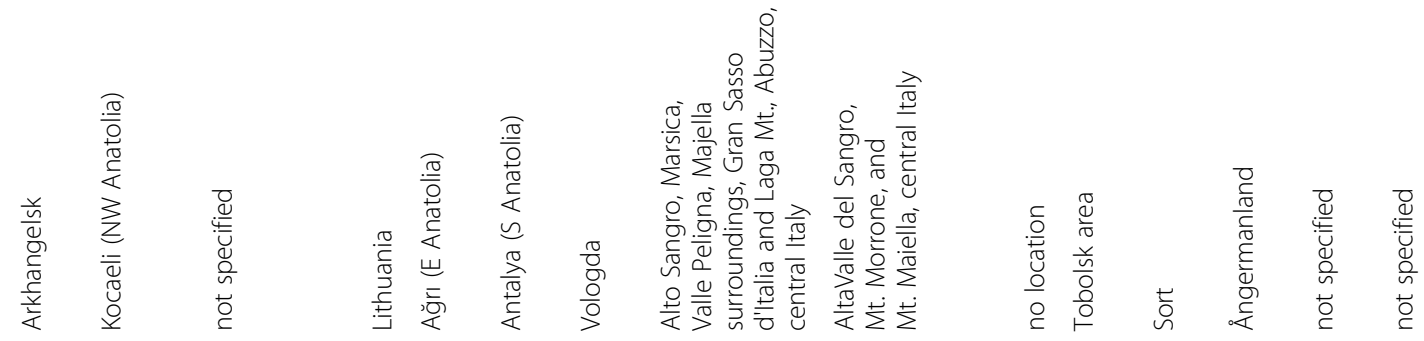

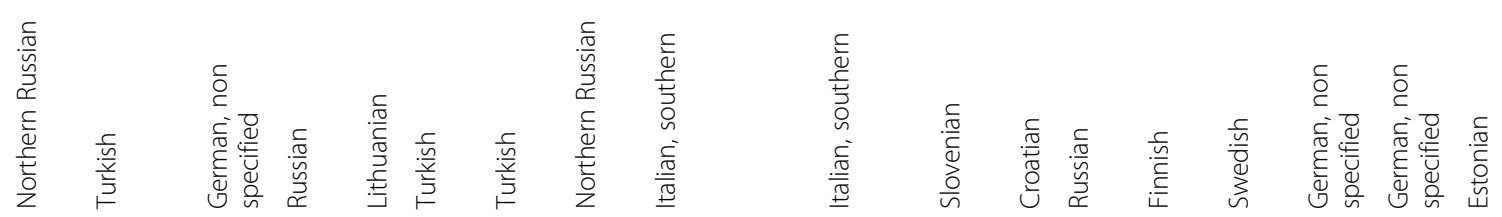

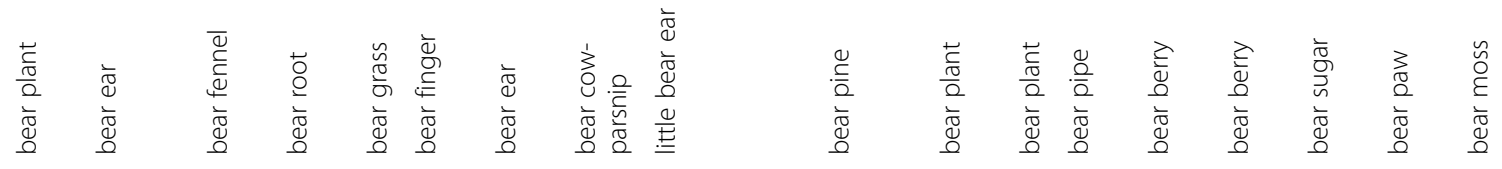

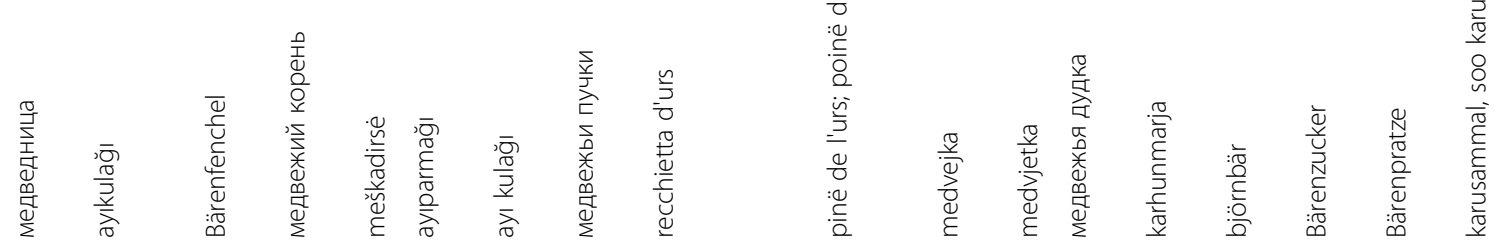

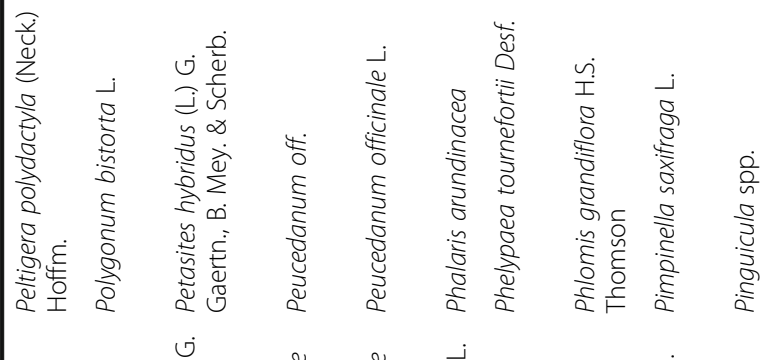

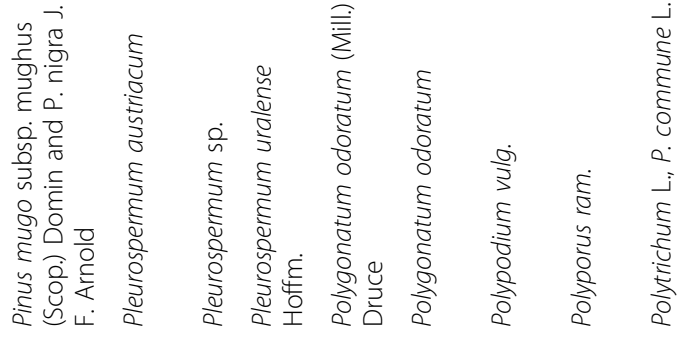

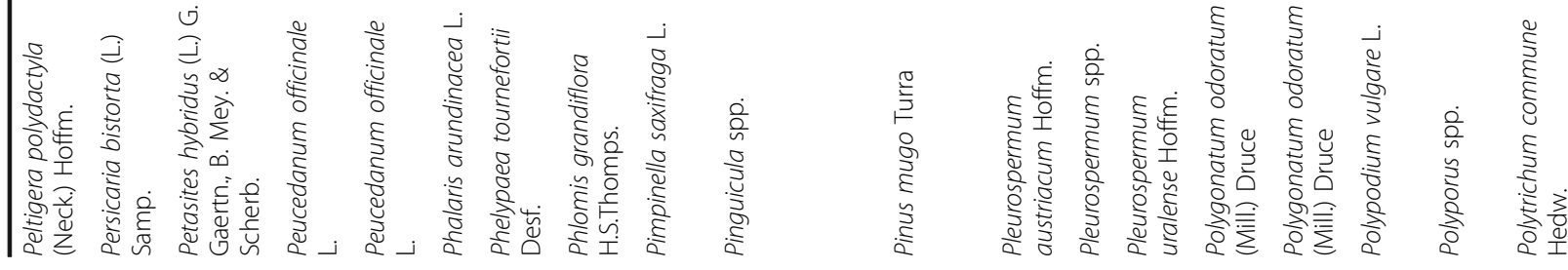




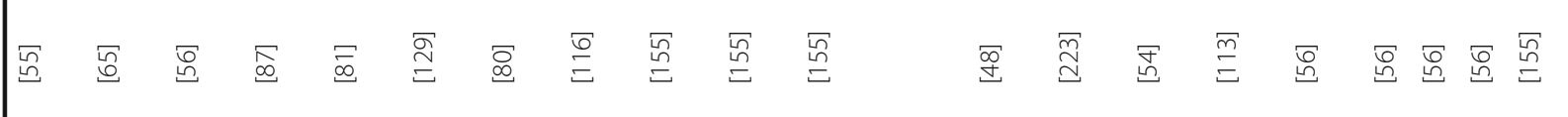

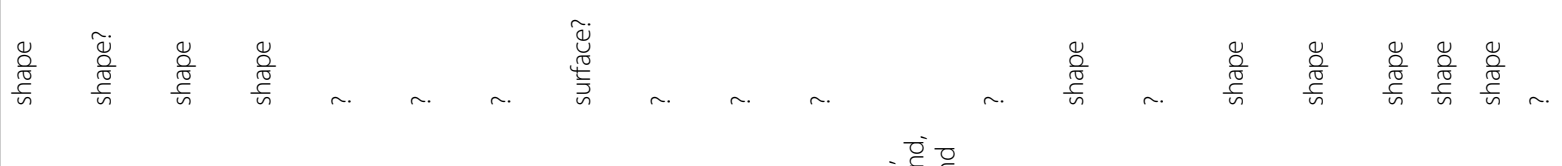

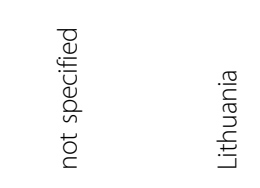
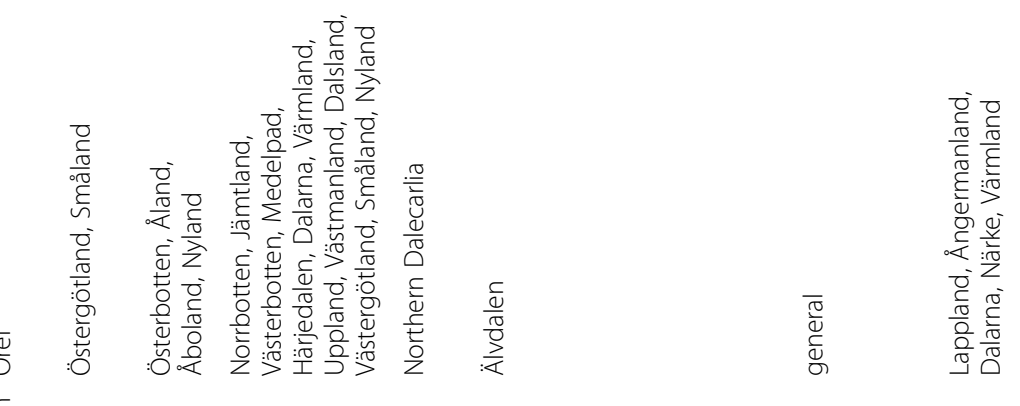

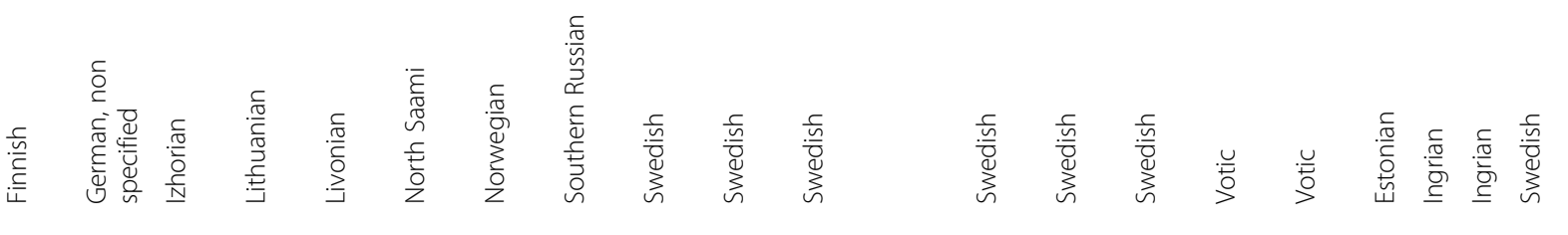

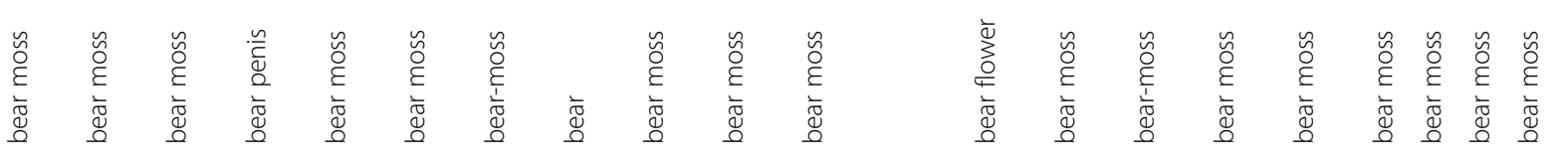

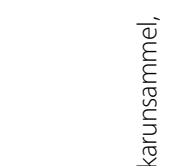

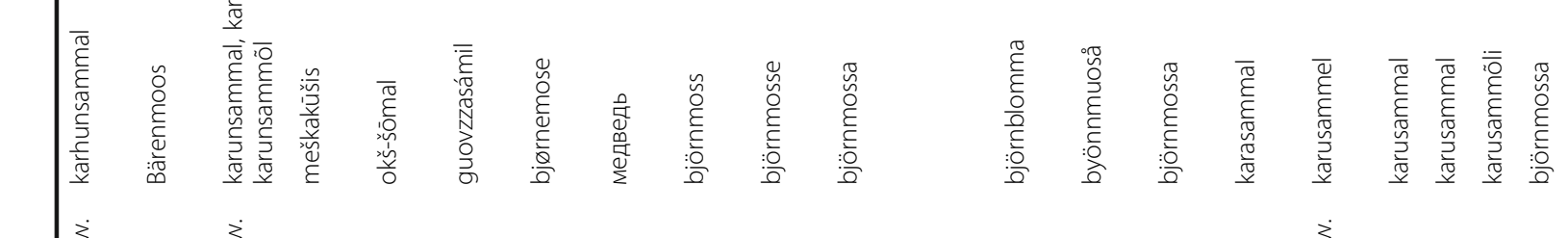

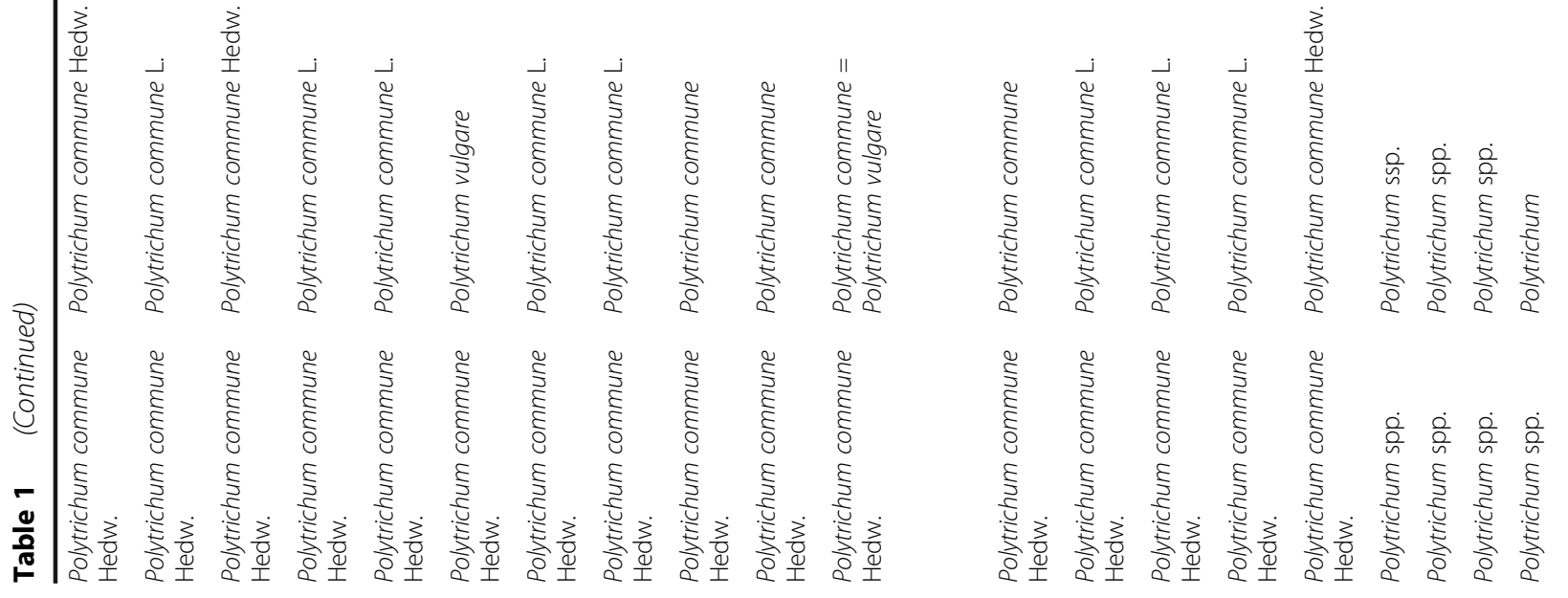




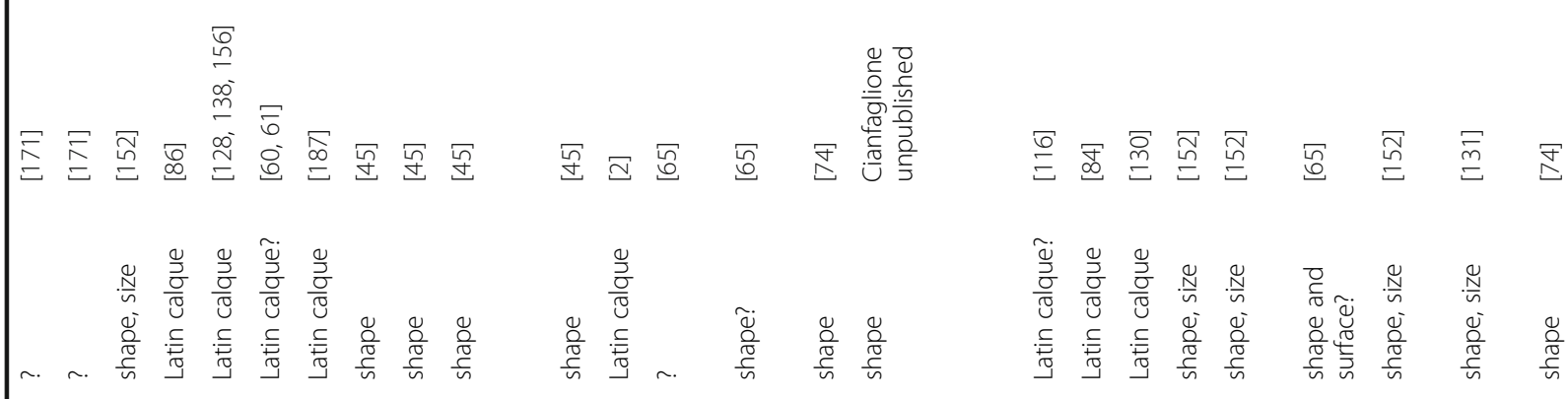

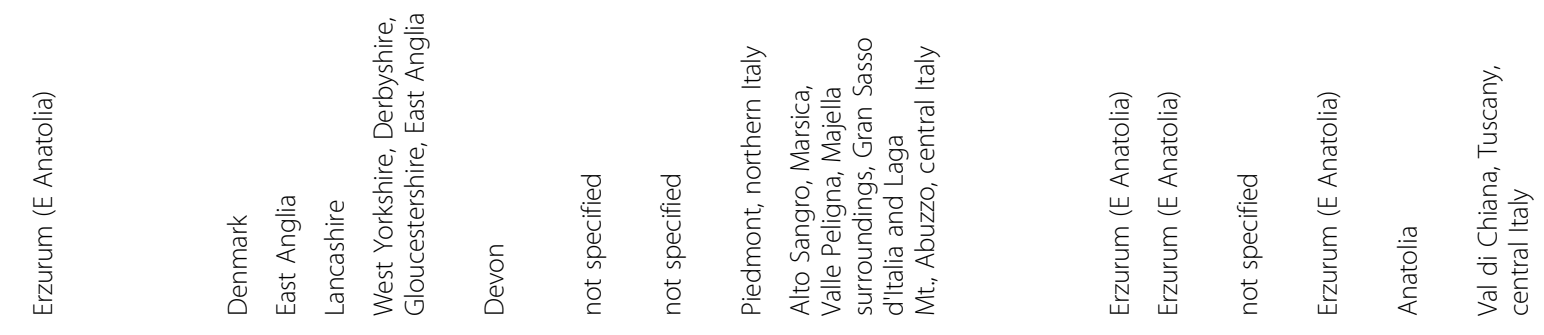

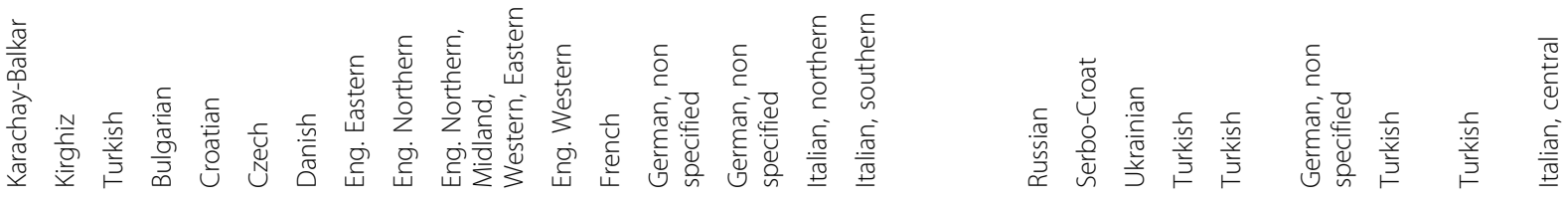

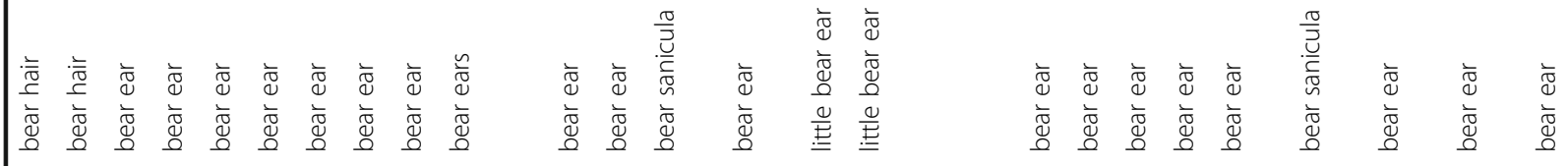

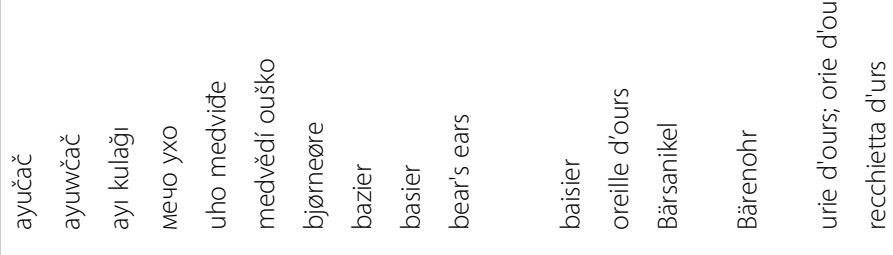

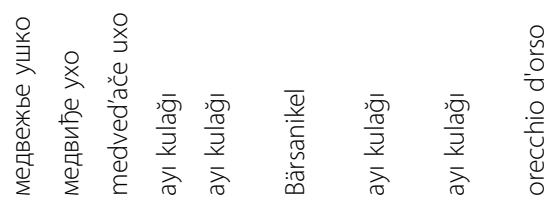

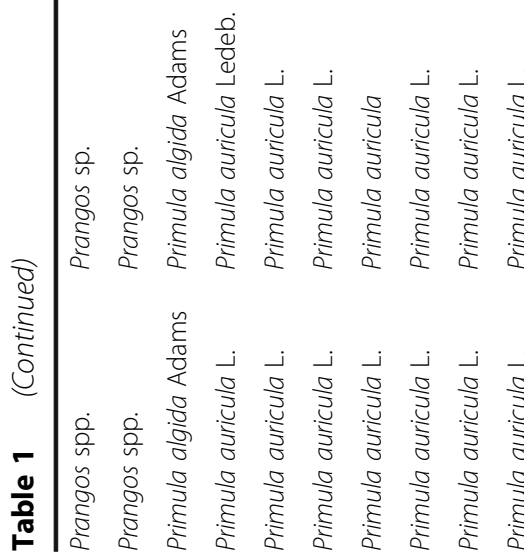

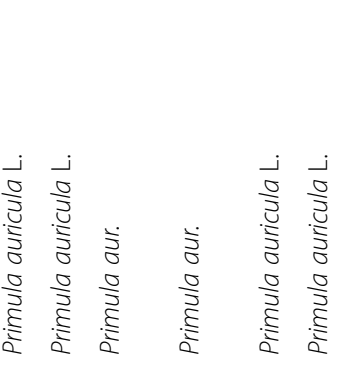

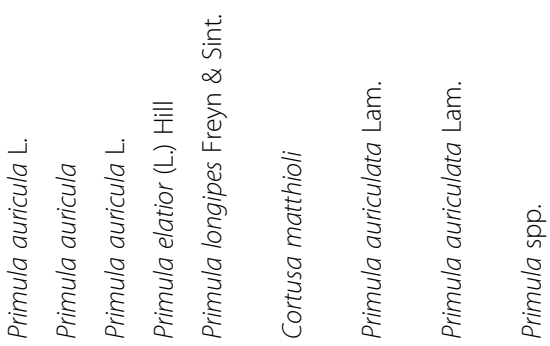

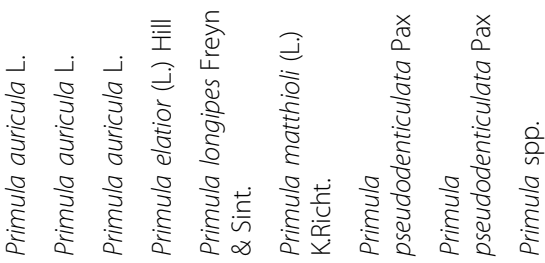




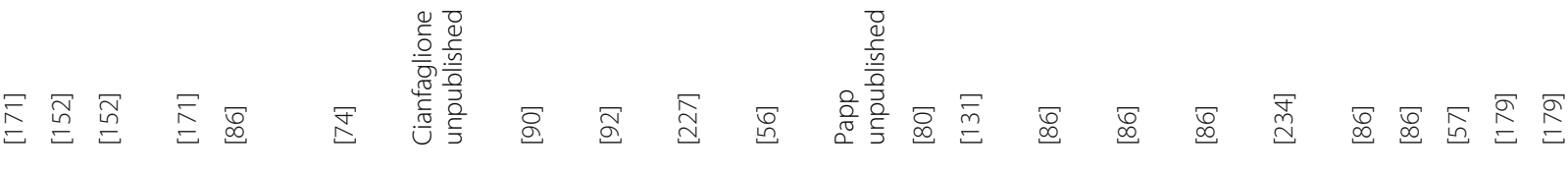

$\frac{\frac{\sqrt{2}}{\frac{2}{2}}}{\frac{2}{2}}$

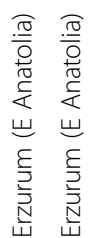
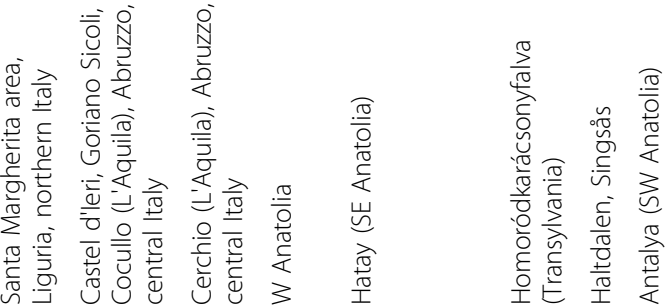

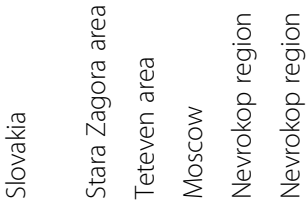

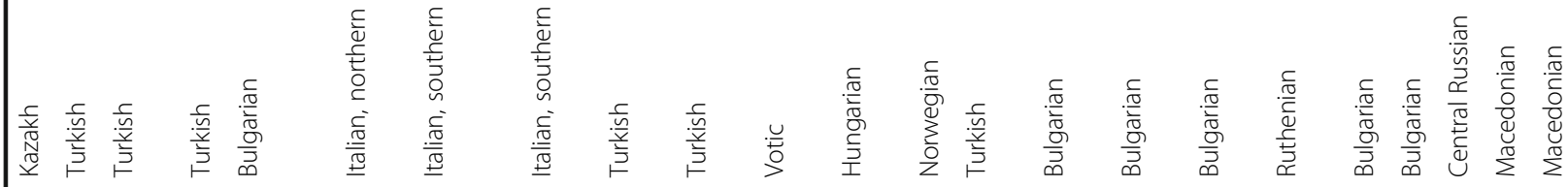

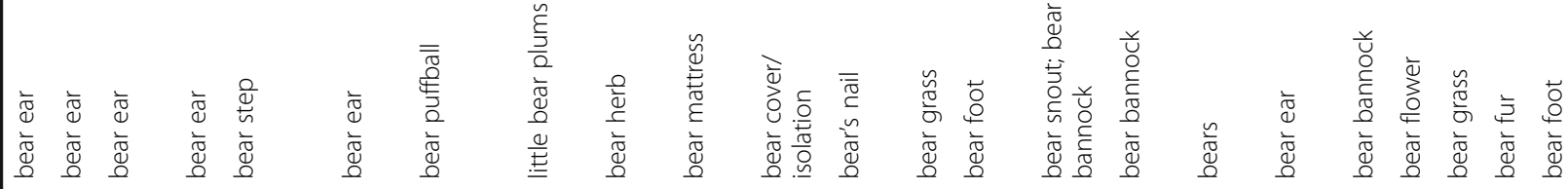

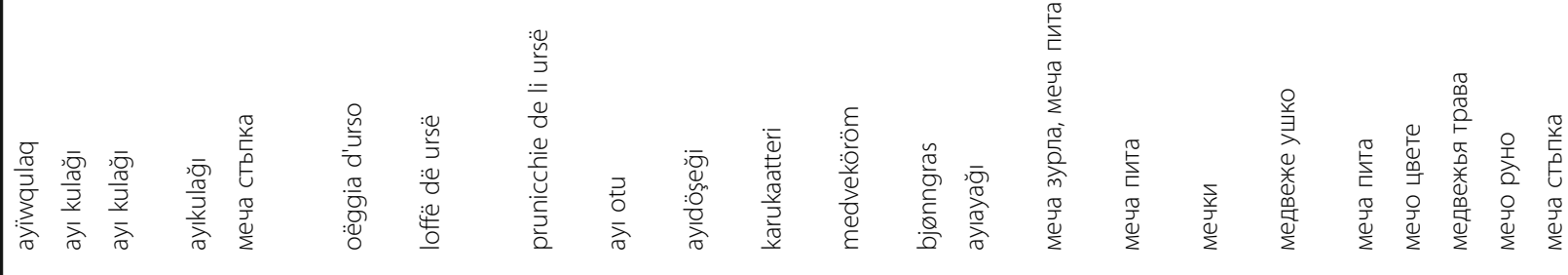

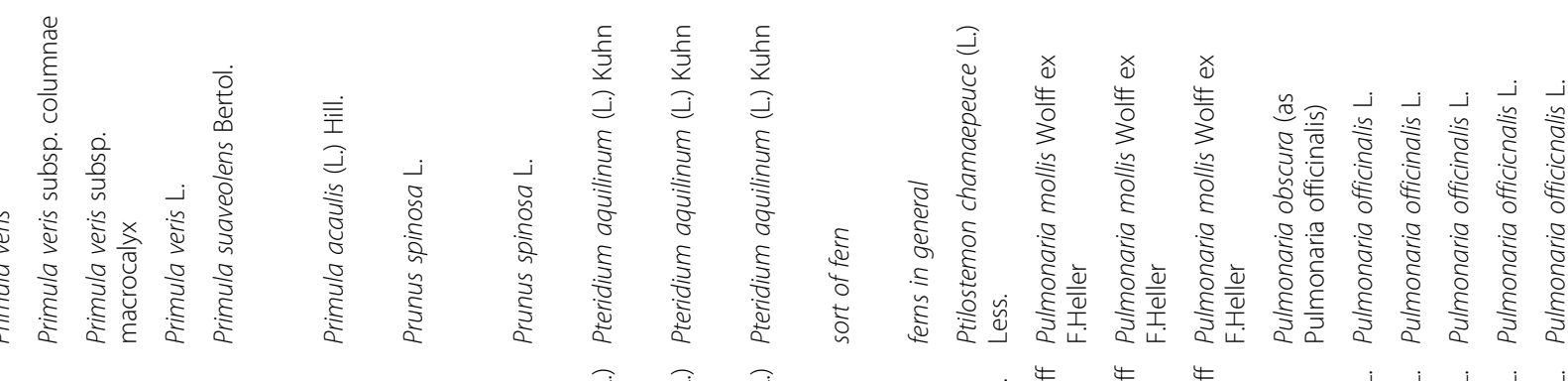




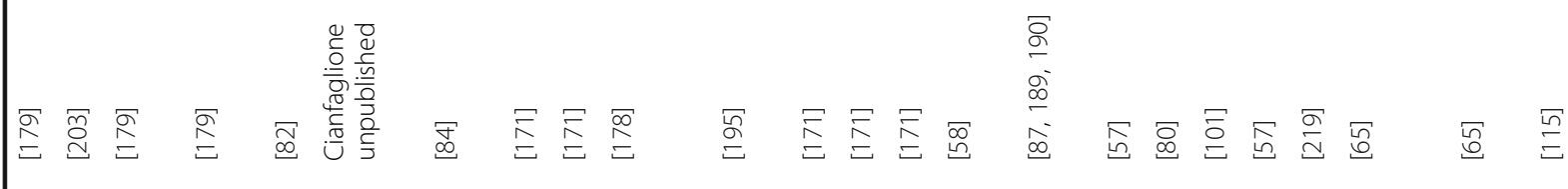

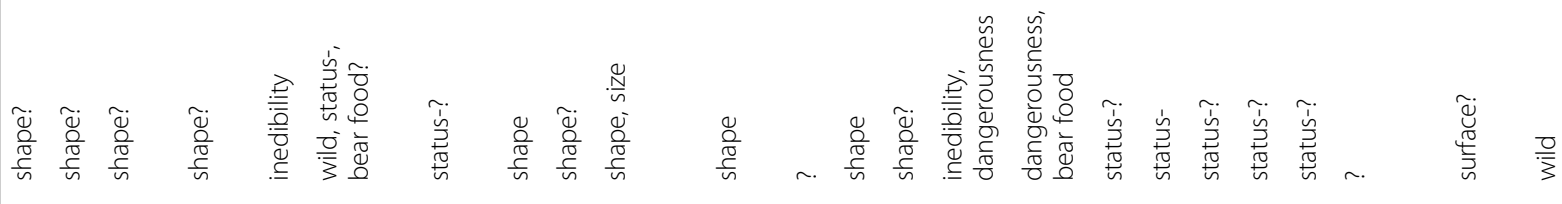

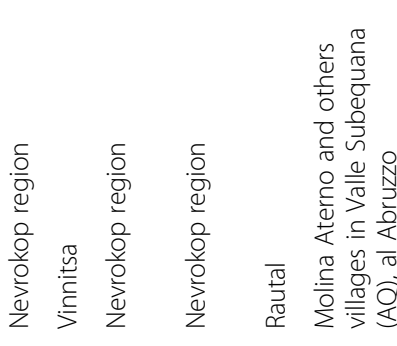
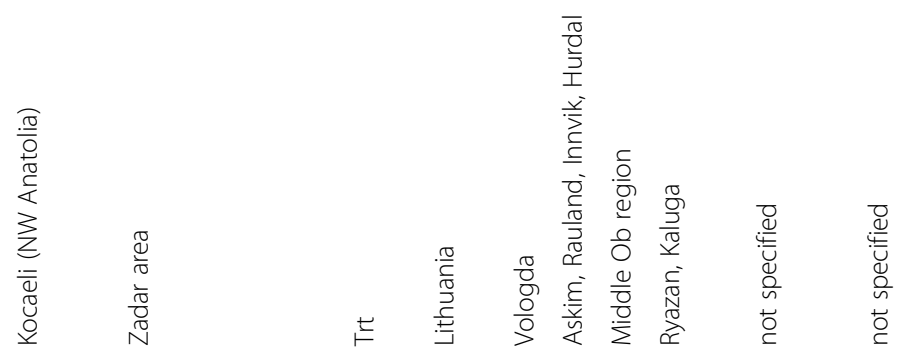

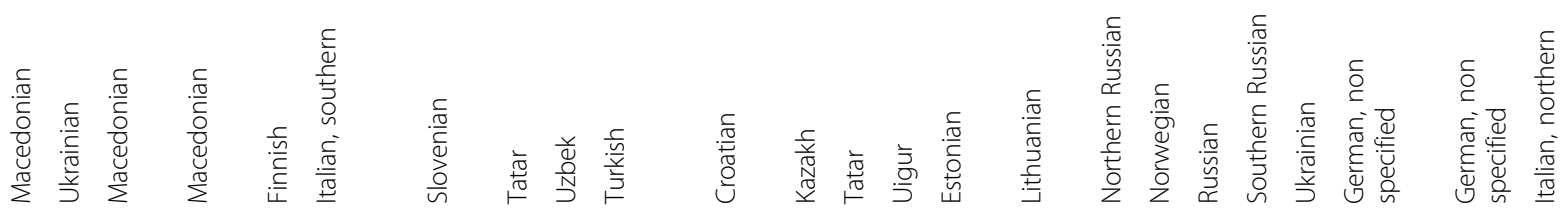

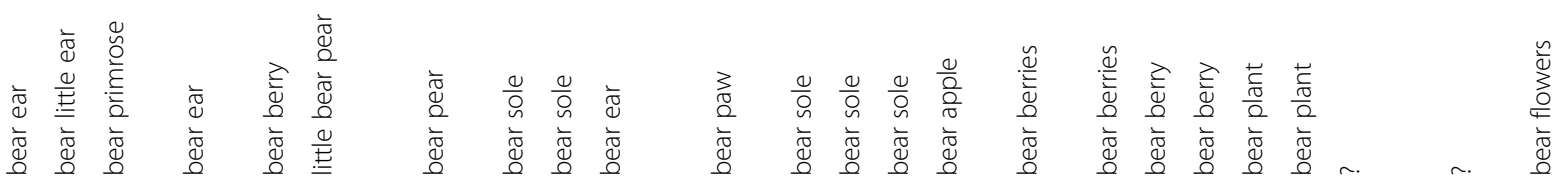

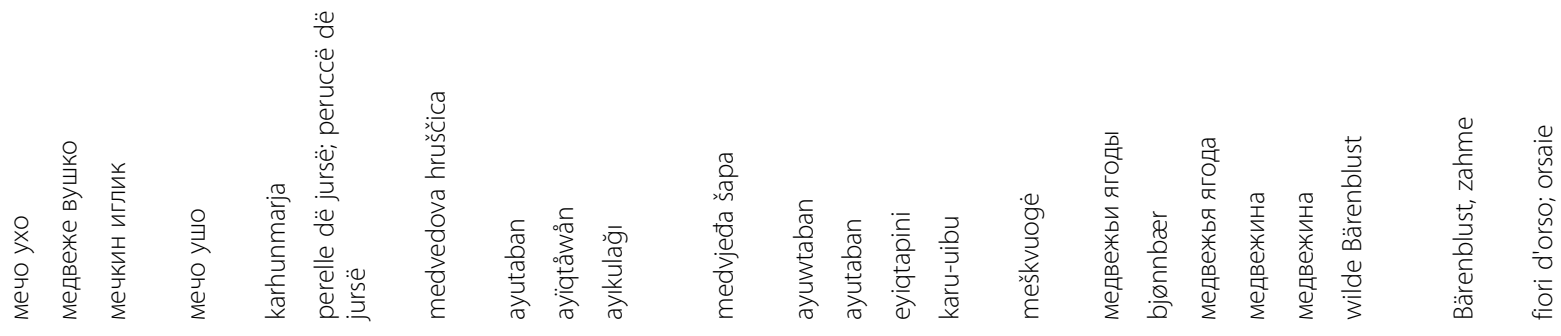

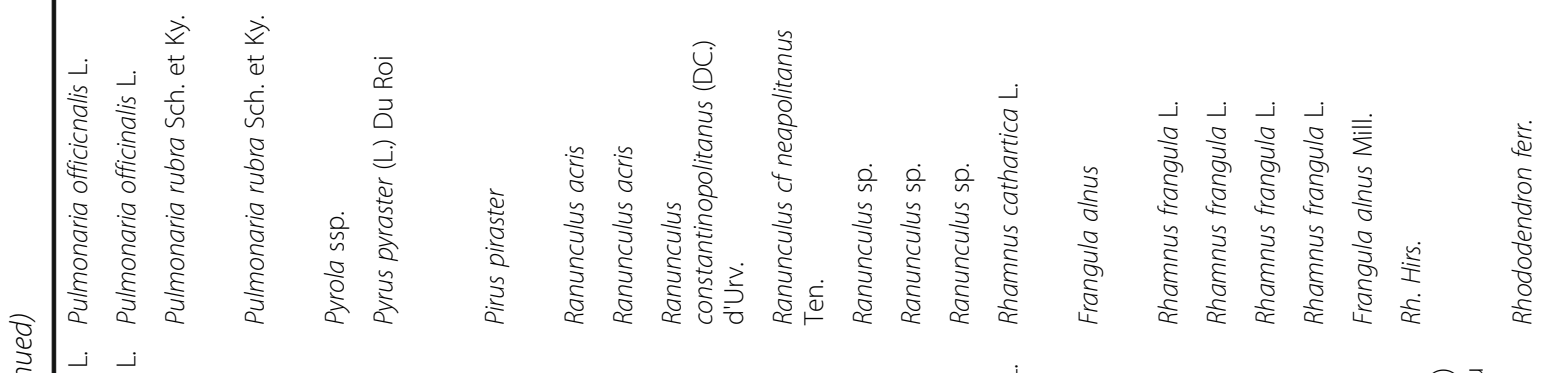

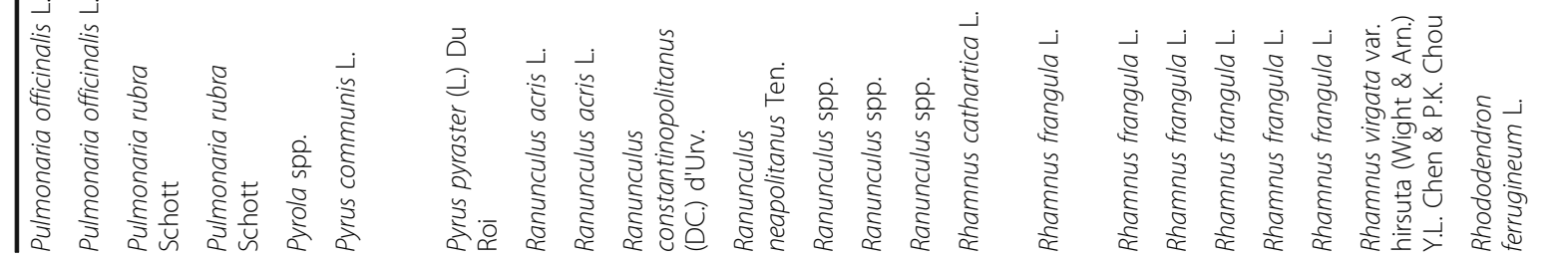




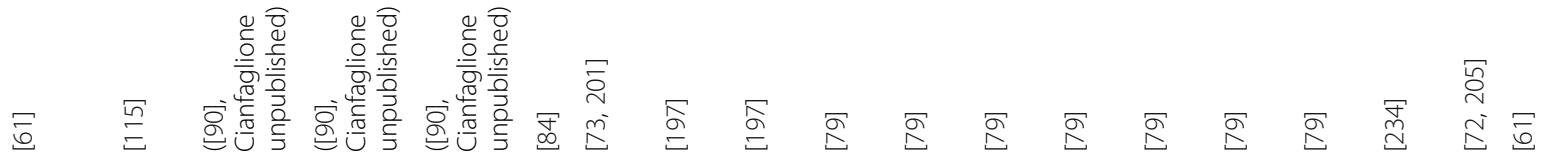

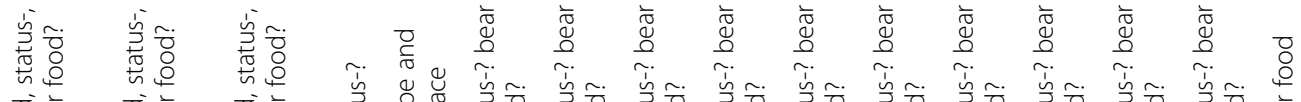

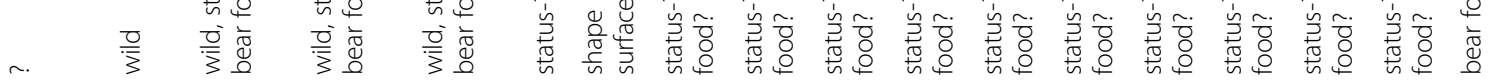

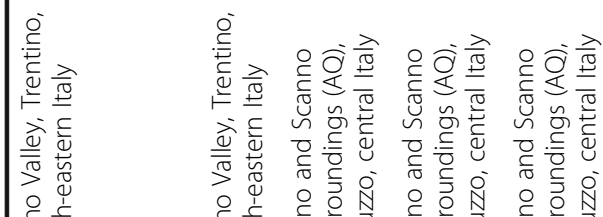

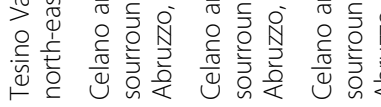

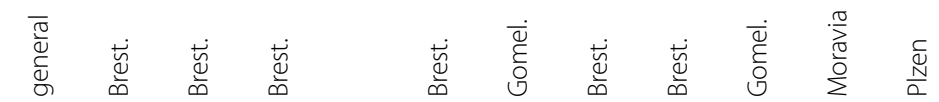

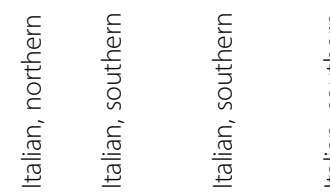

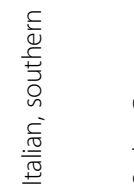

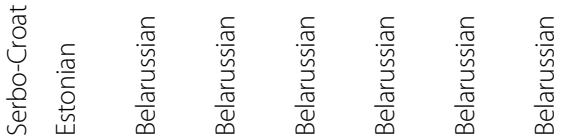

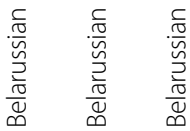

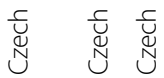

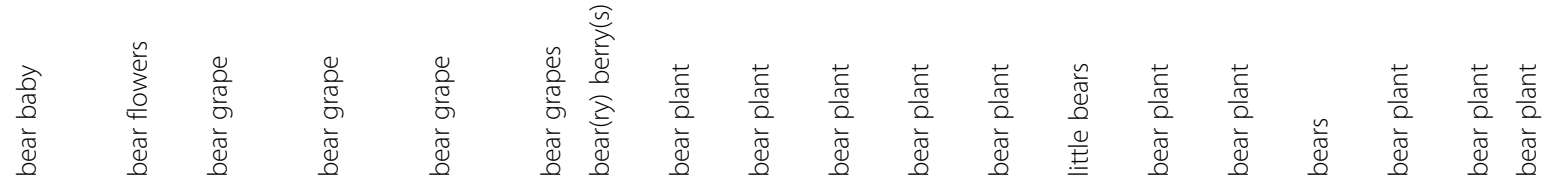

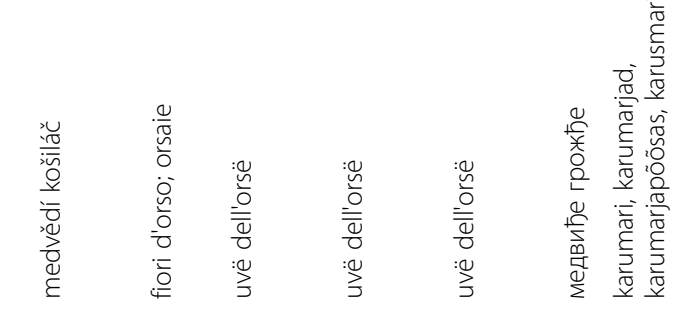
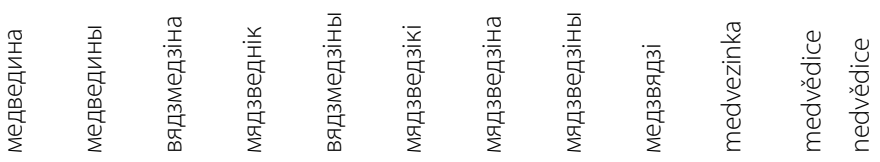

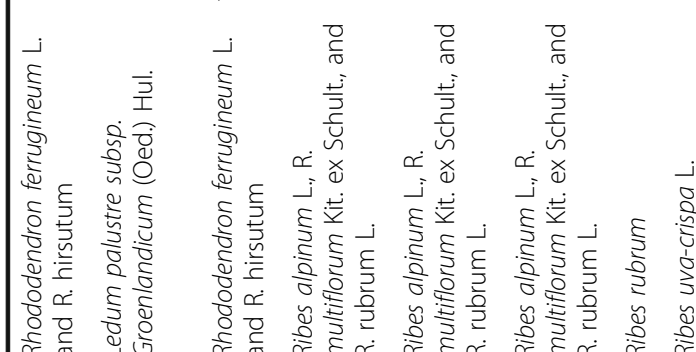

这 


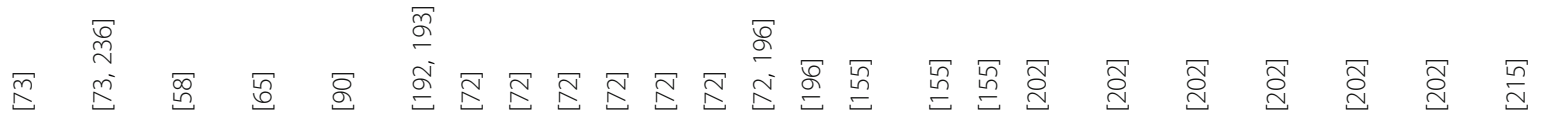

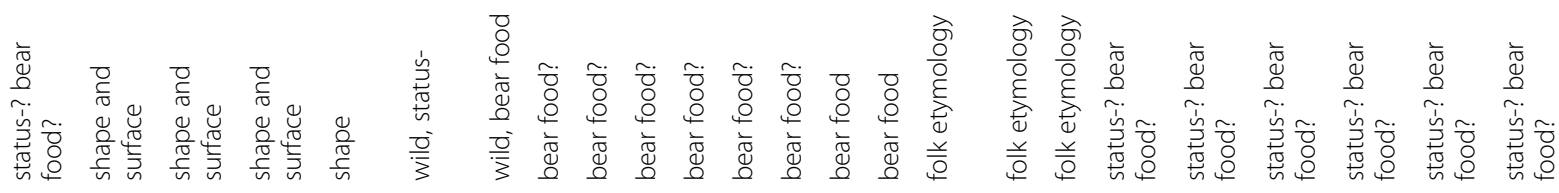

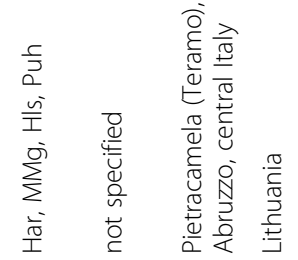

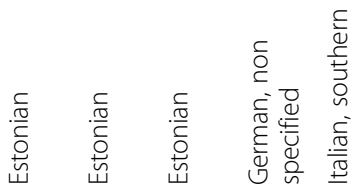

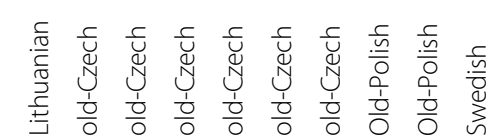

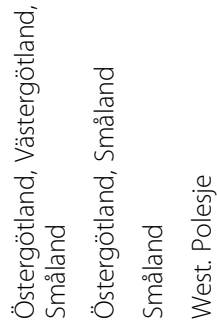

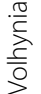

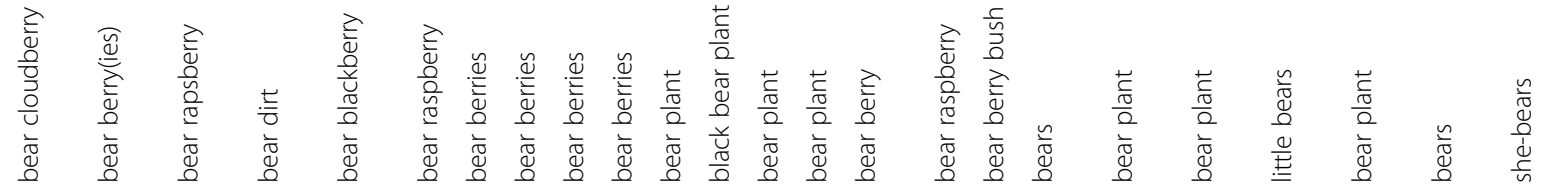

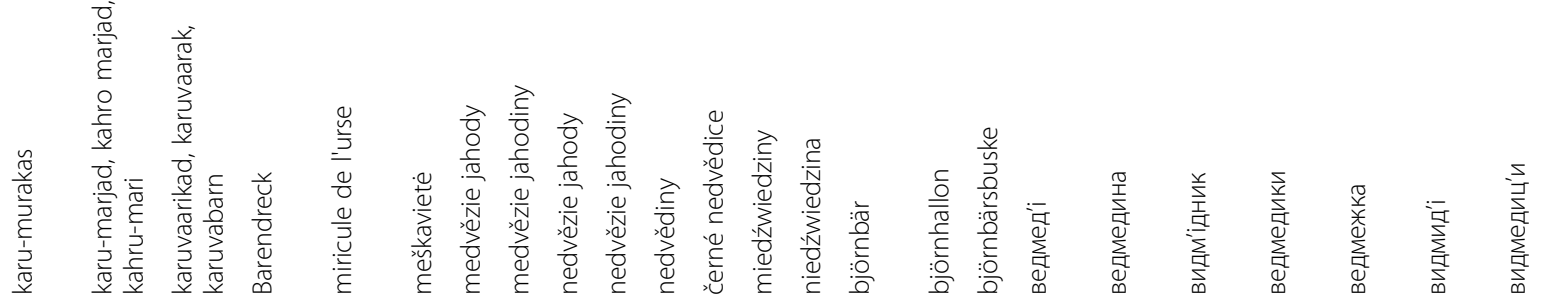




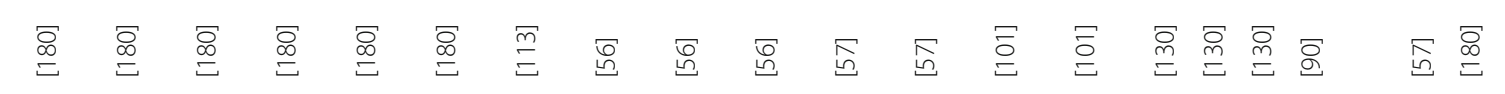

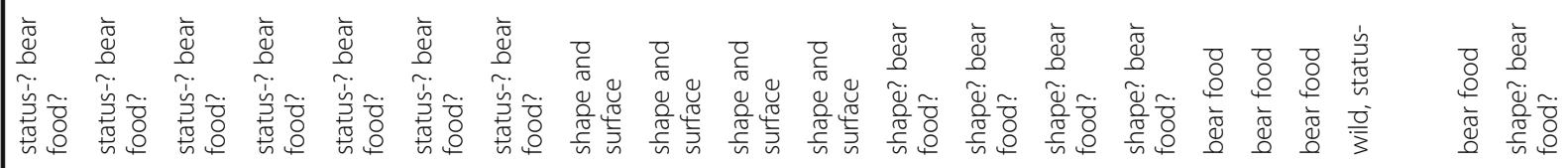

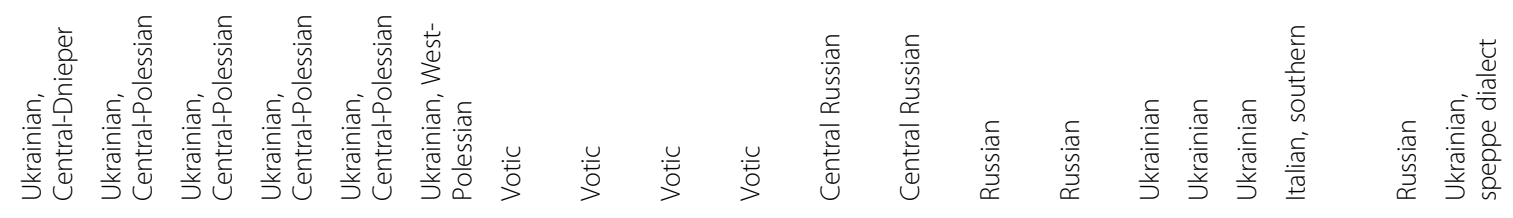

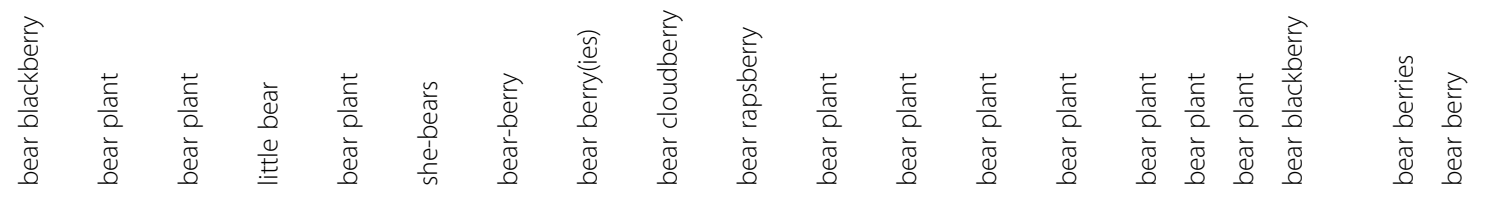

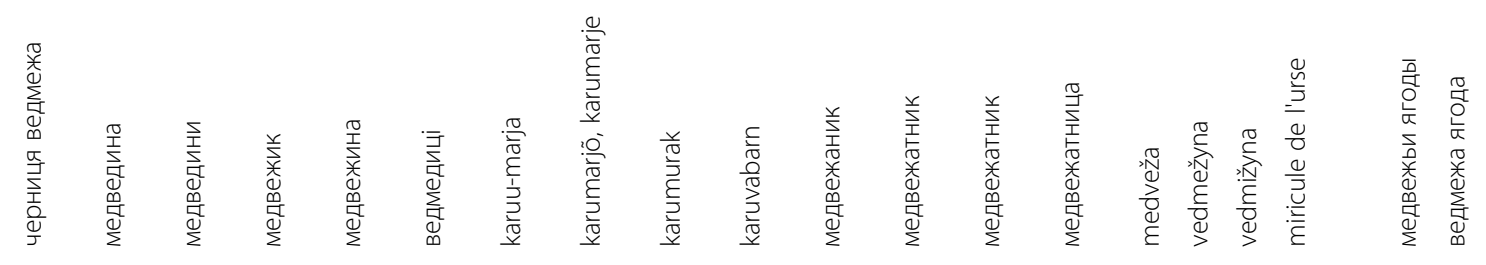

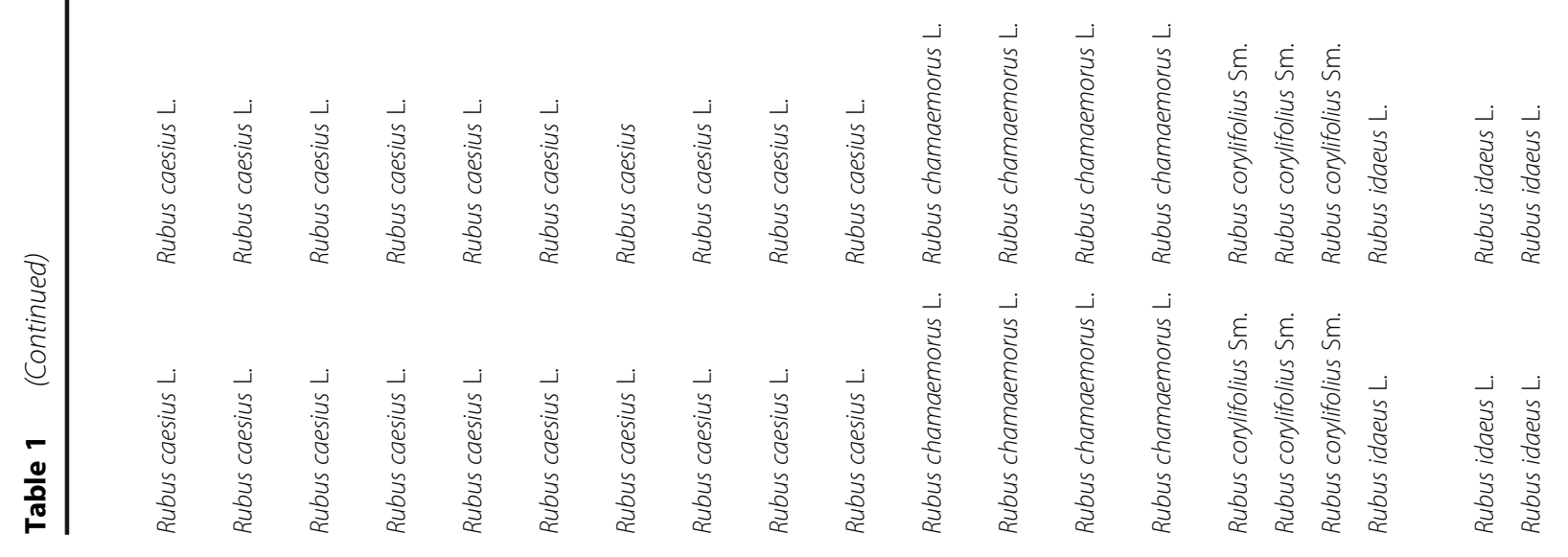




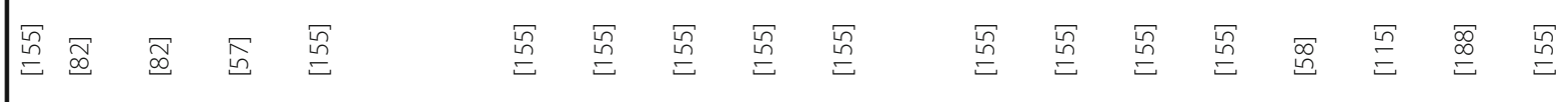

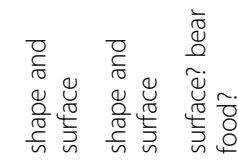
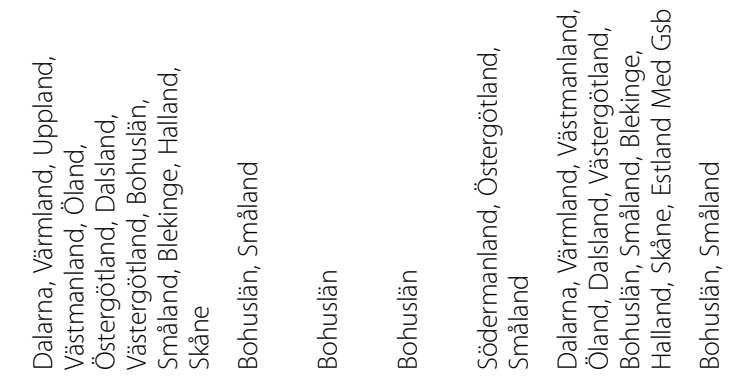

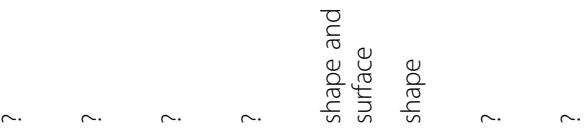

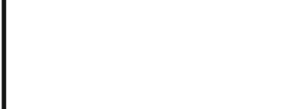

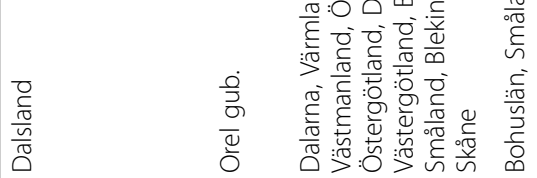
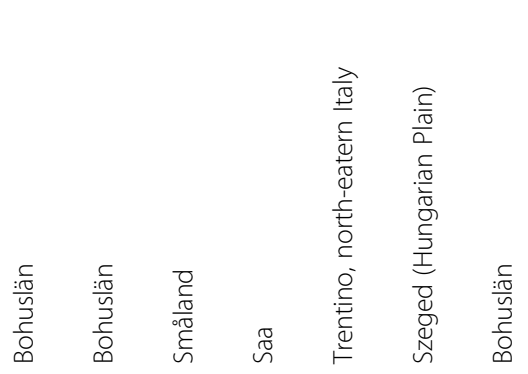

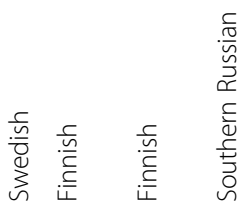

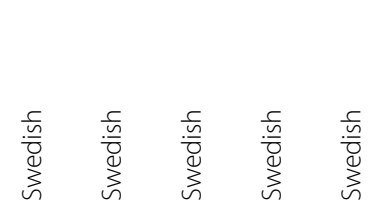

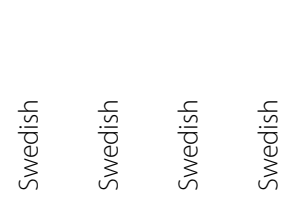

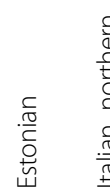

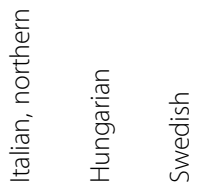

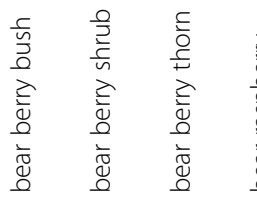

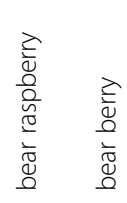

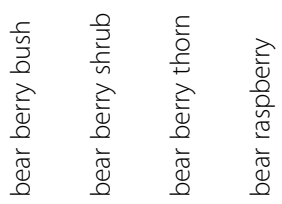

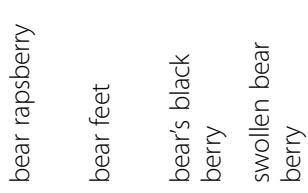

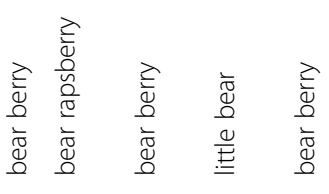

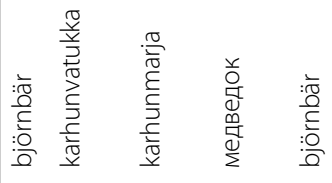

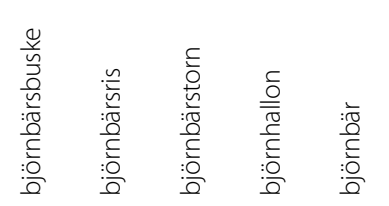

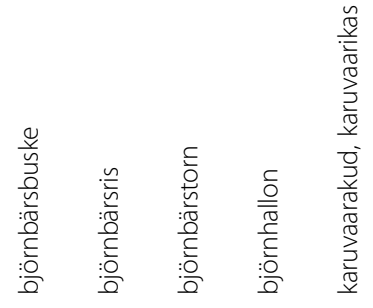
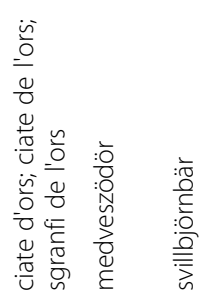

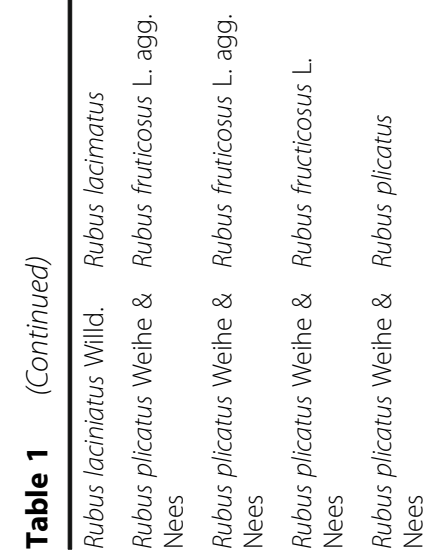

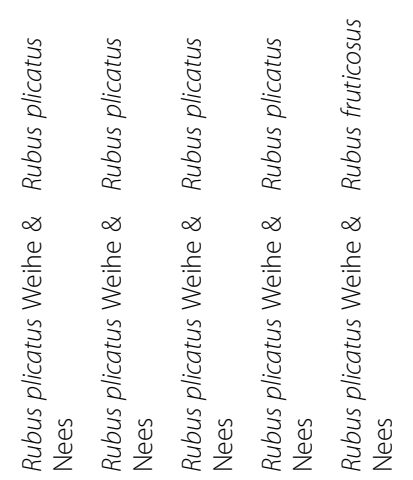

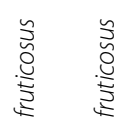

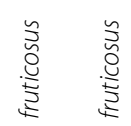

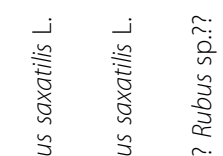

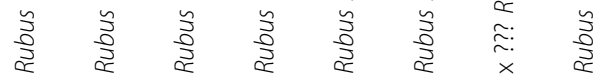

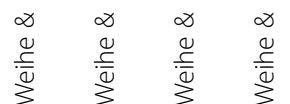

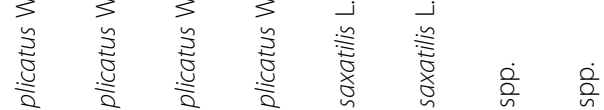

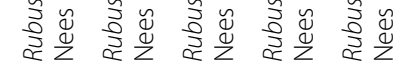

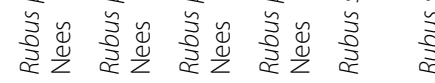




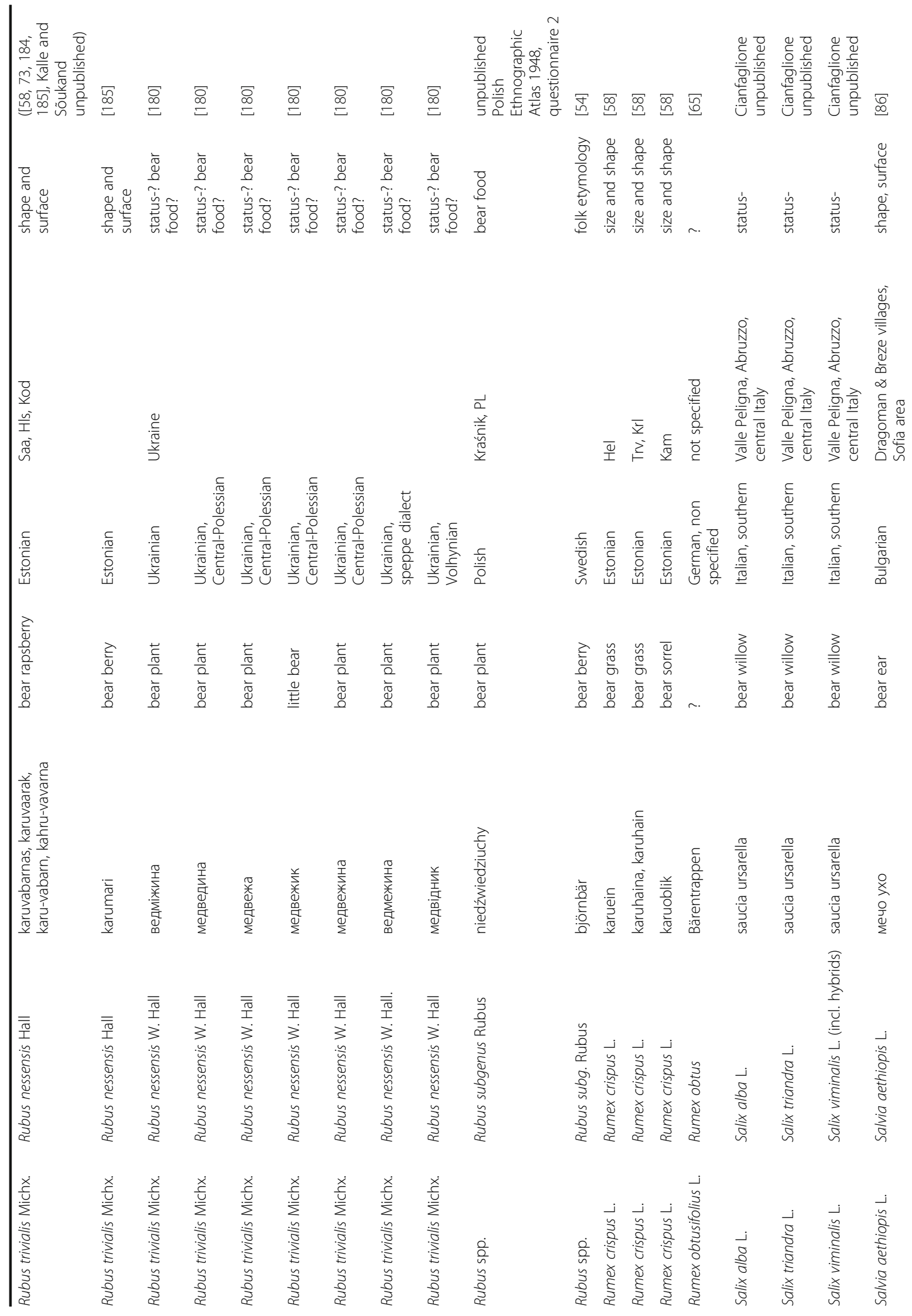




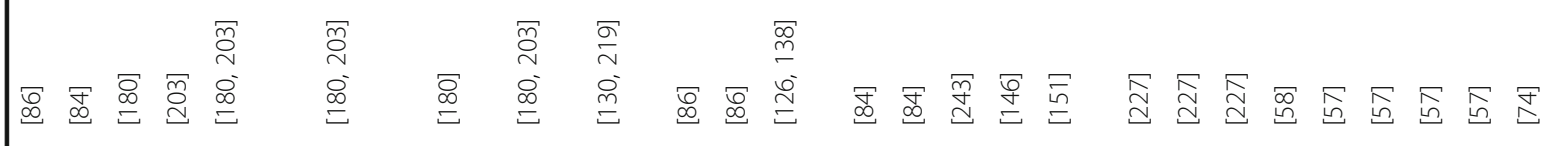

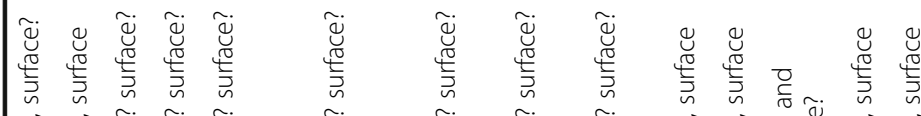

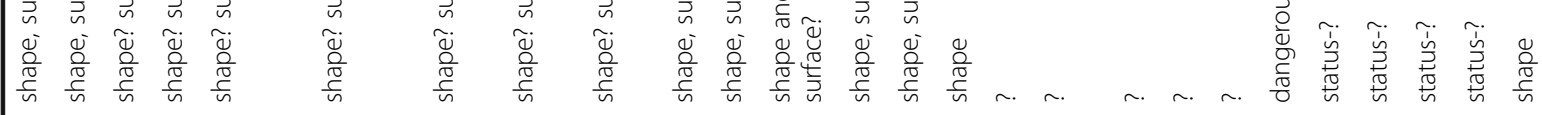

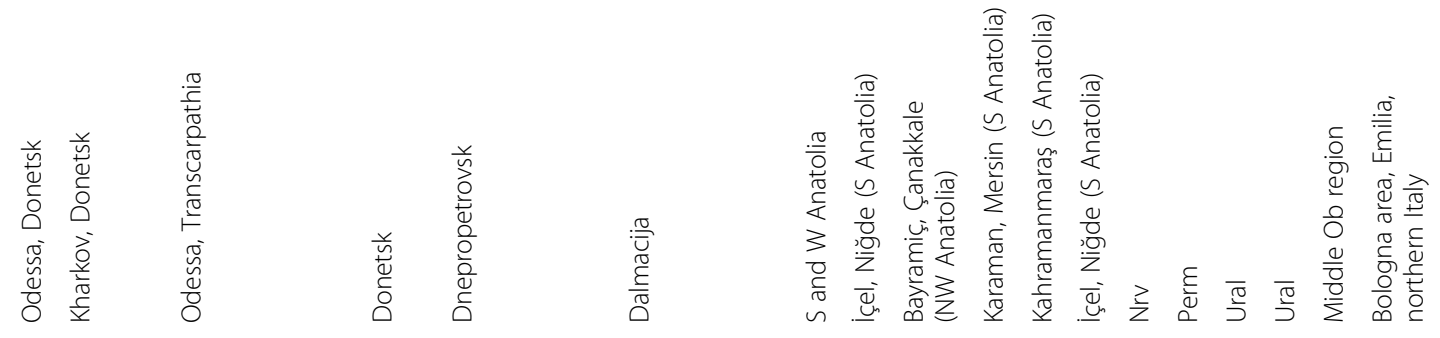

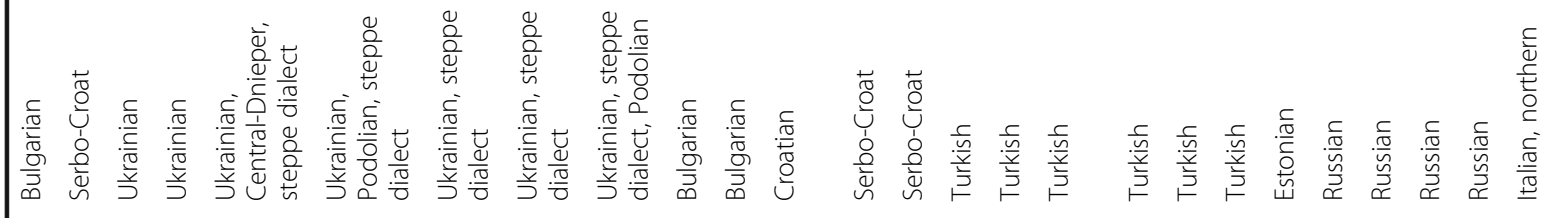

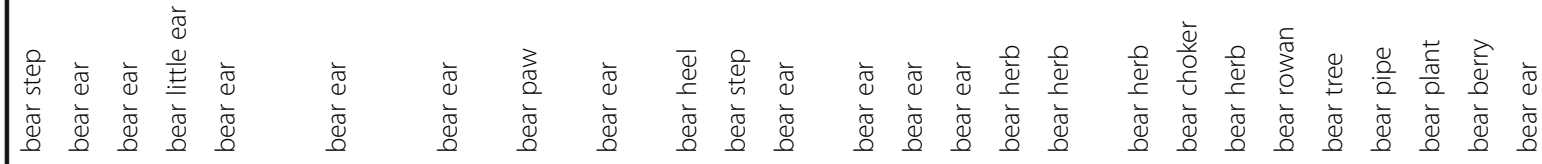

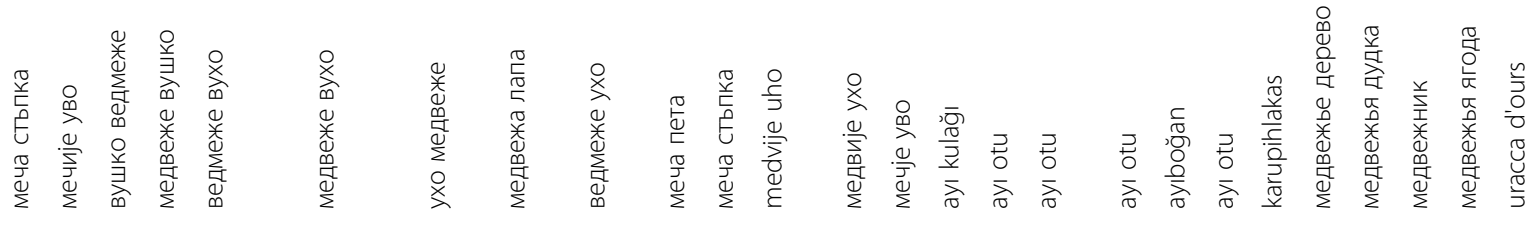




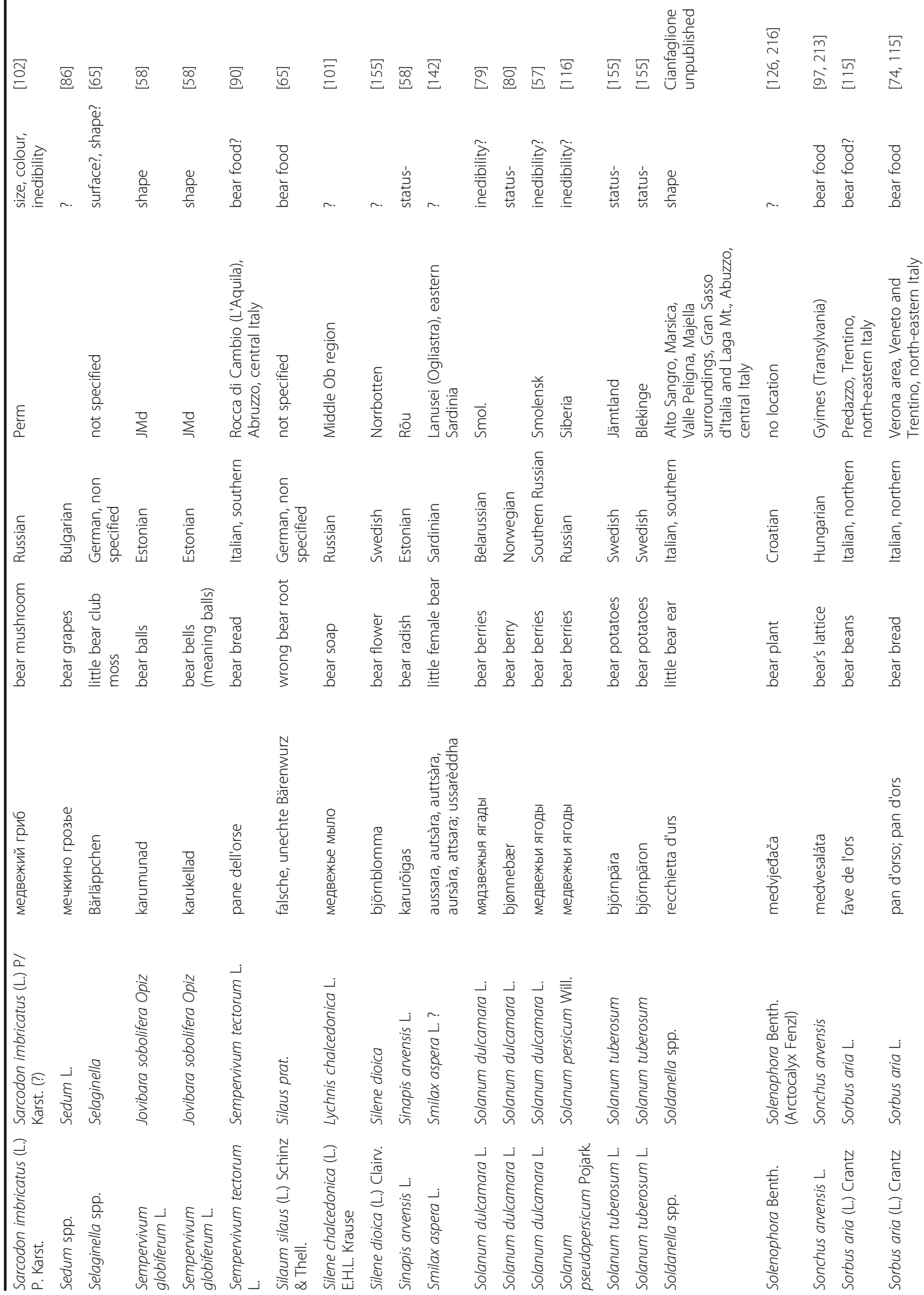




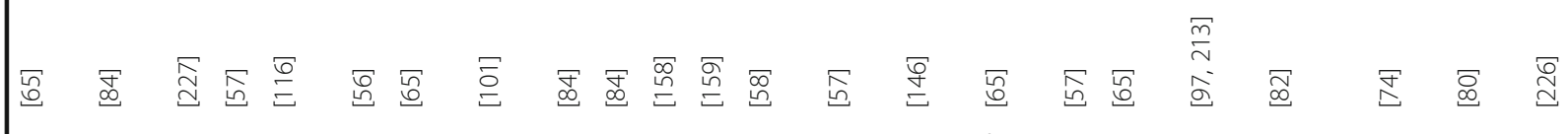

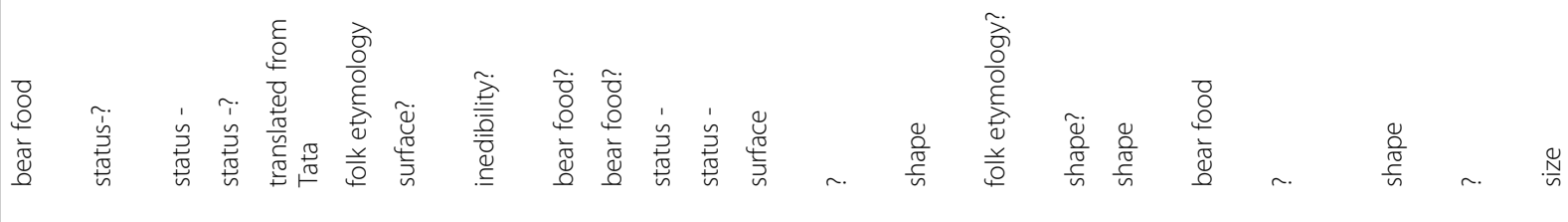

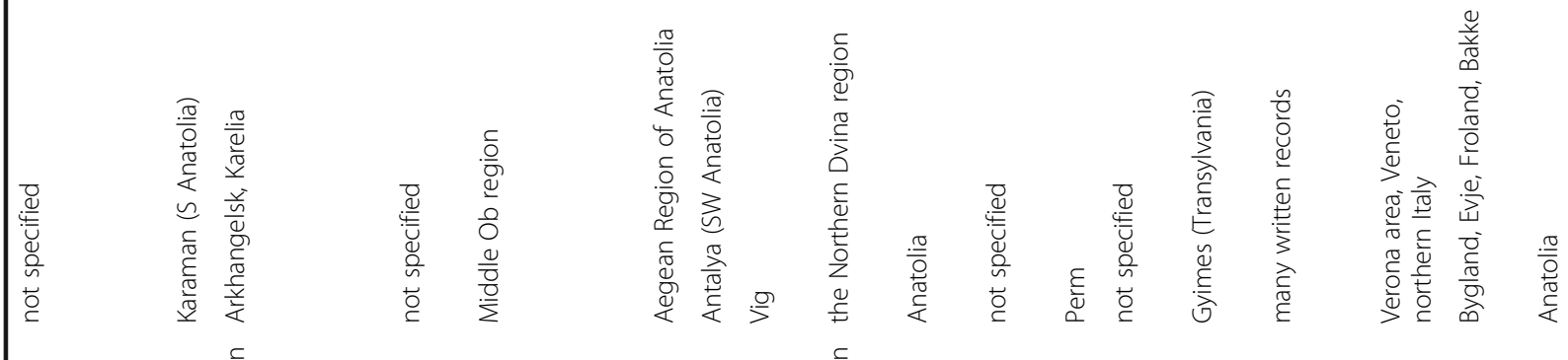

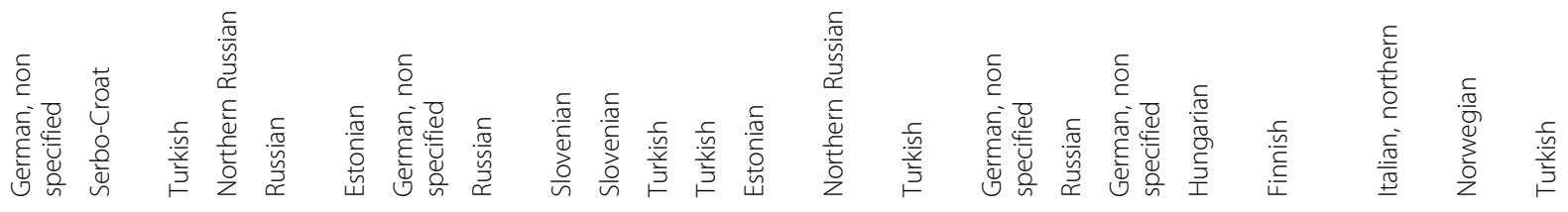

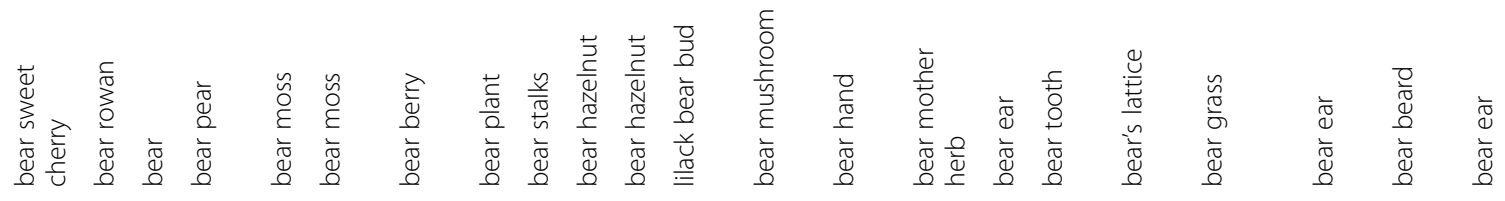

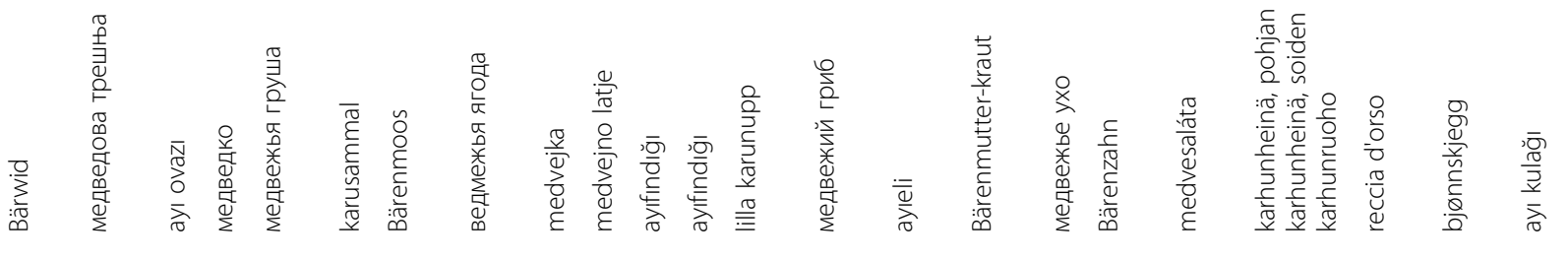

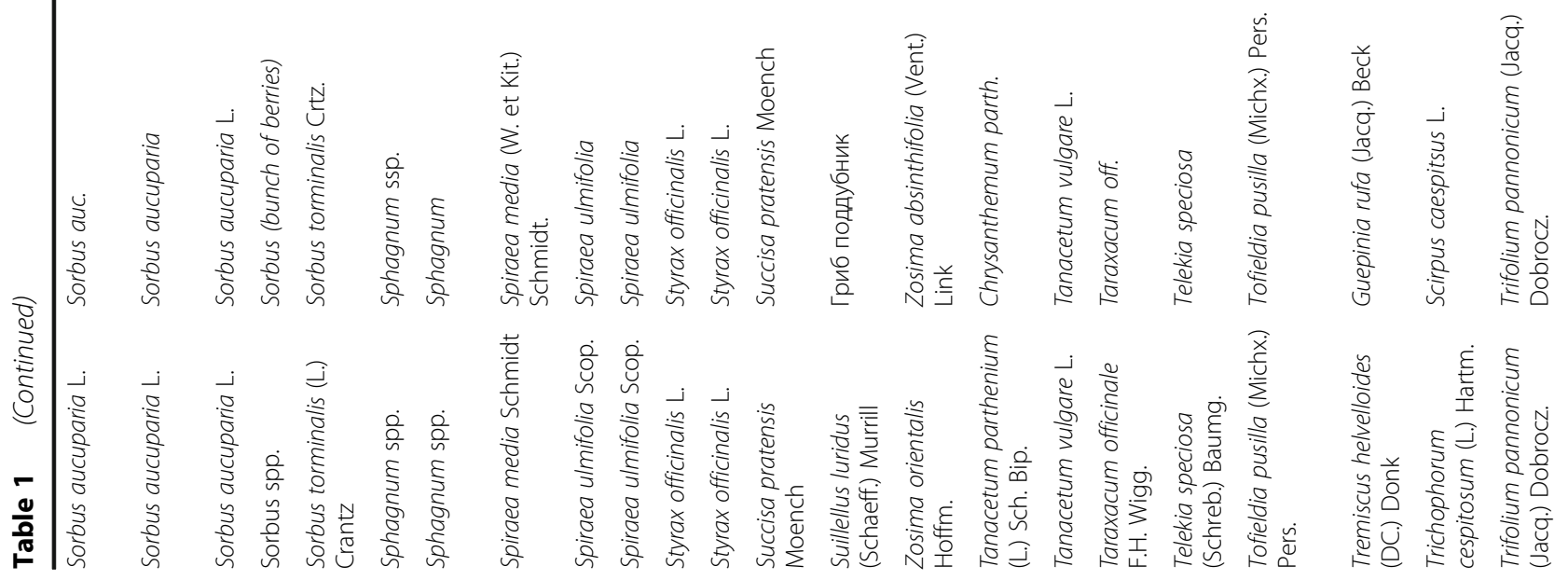




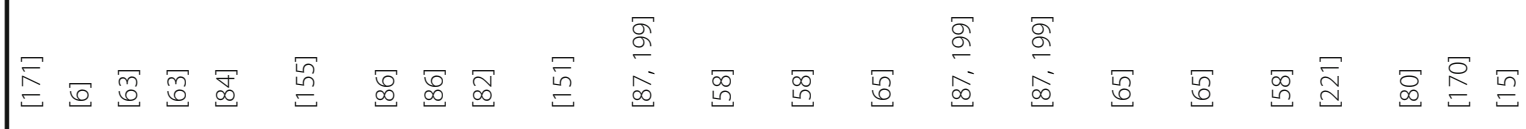

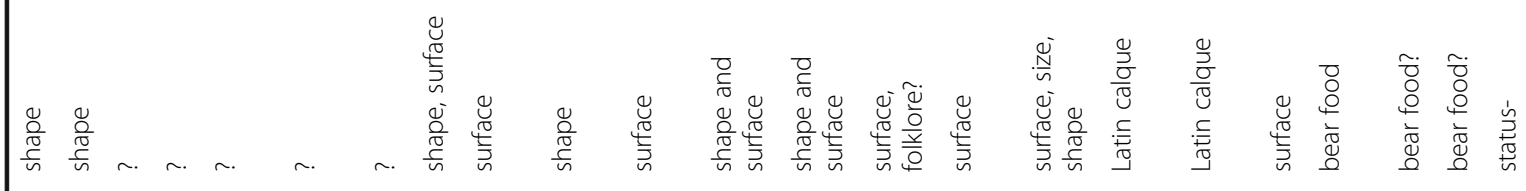<smiles>CC(C)CC(C)C</smiles>

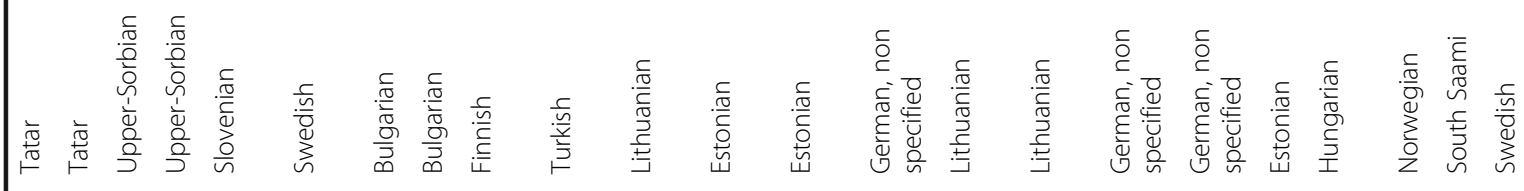

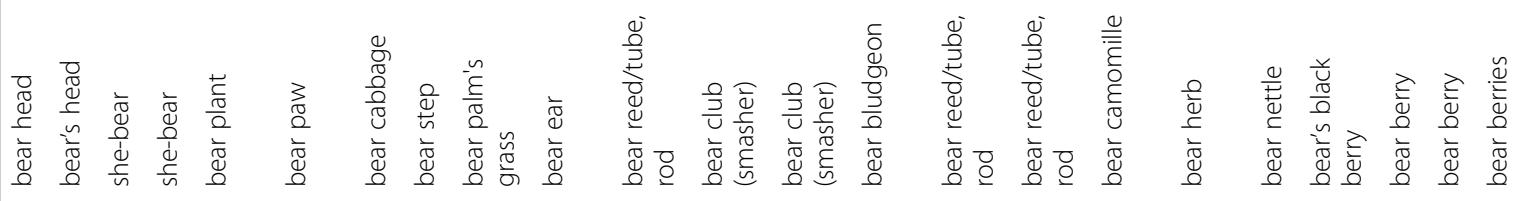

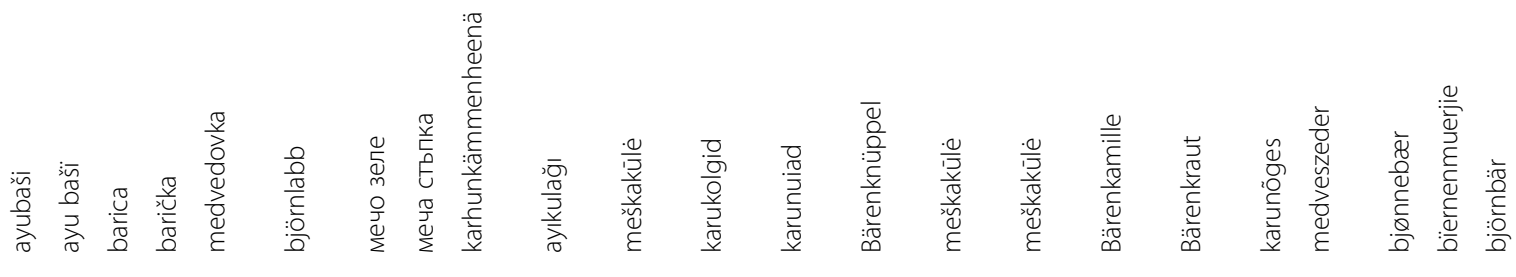

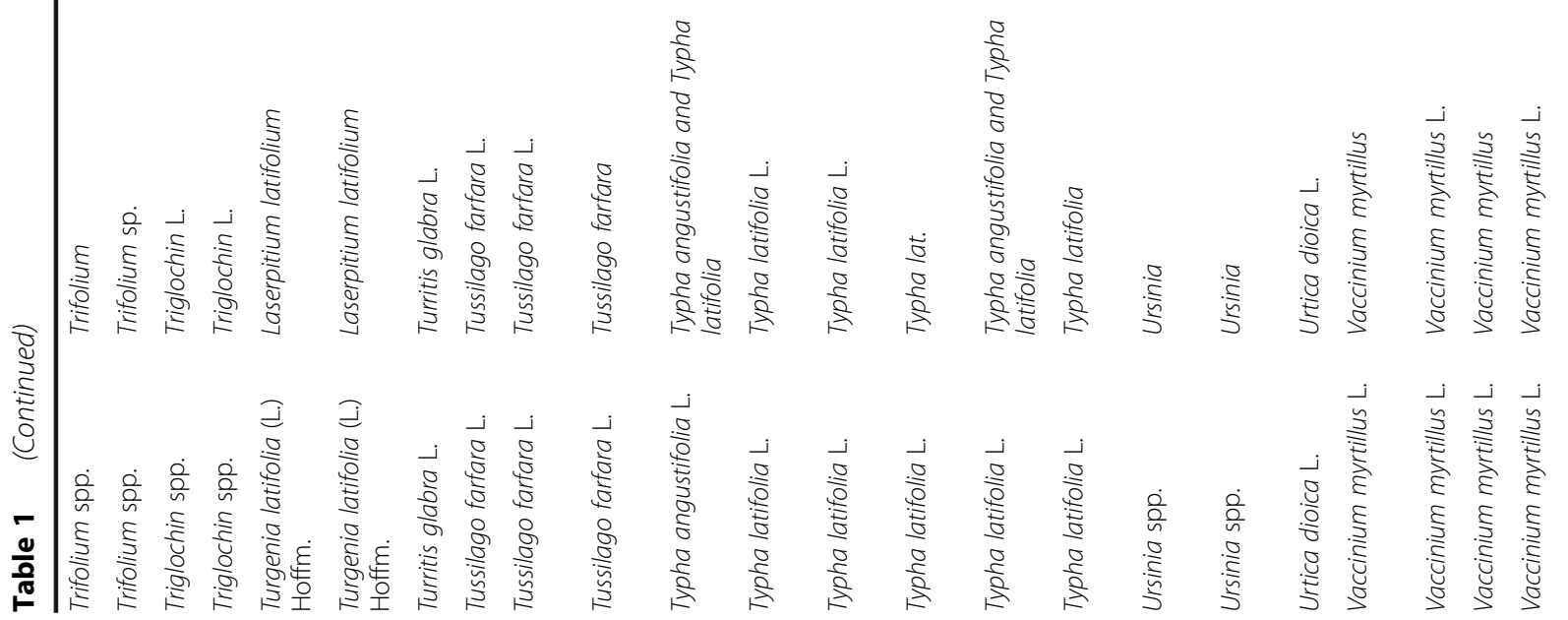




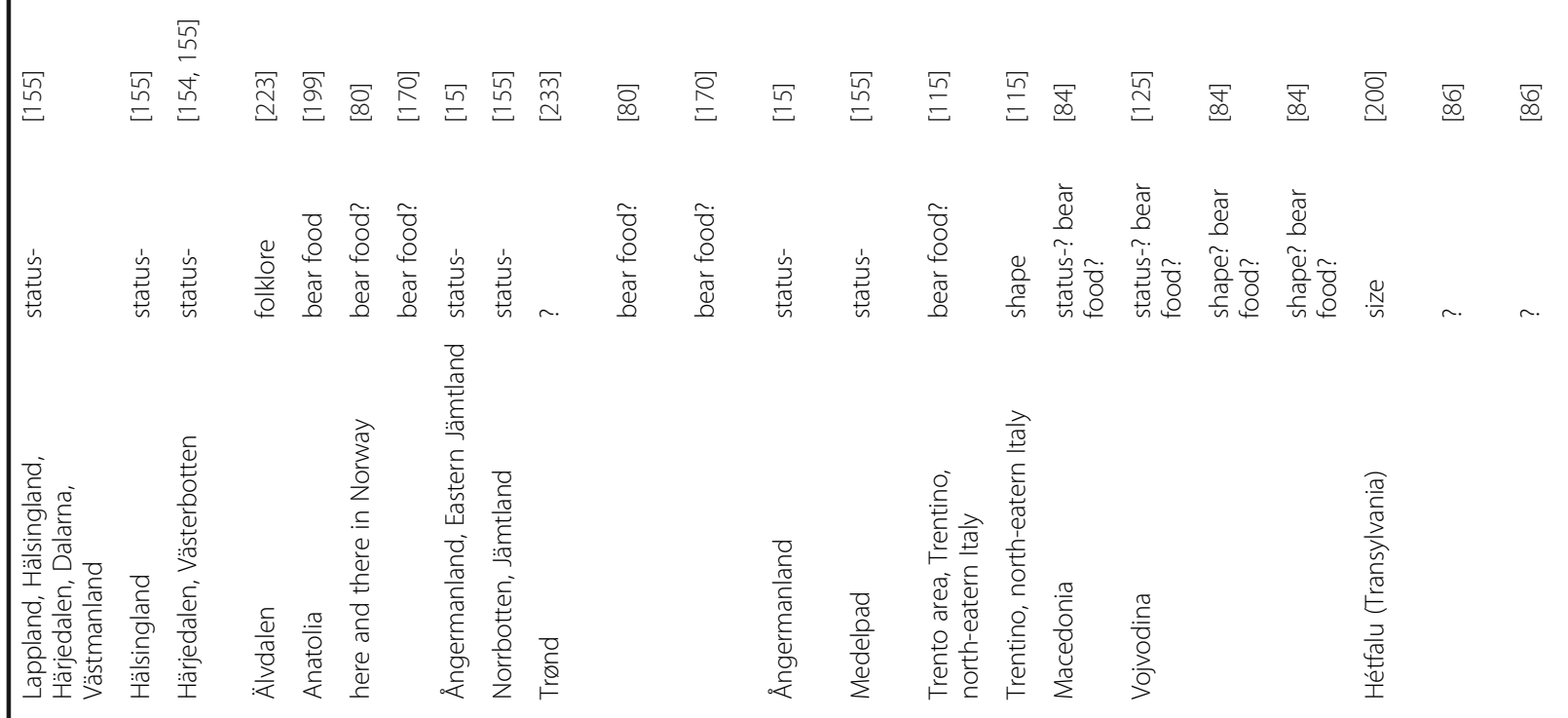

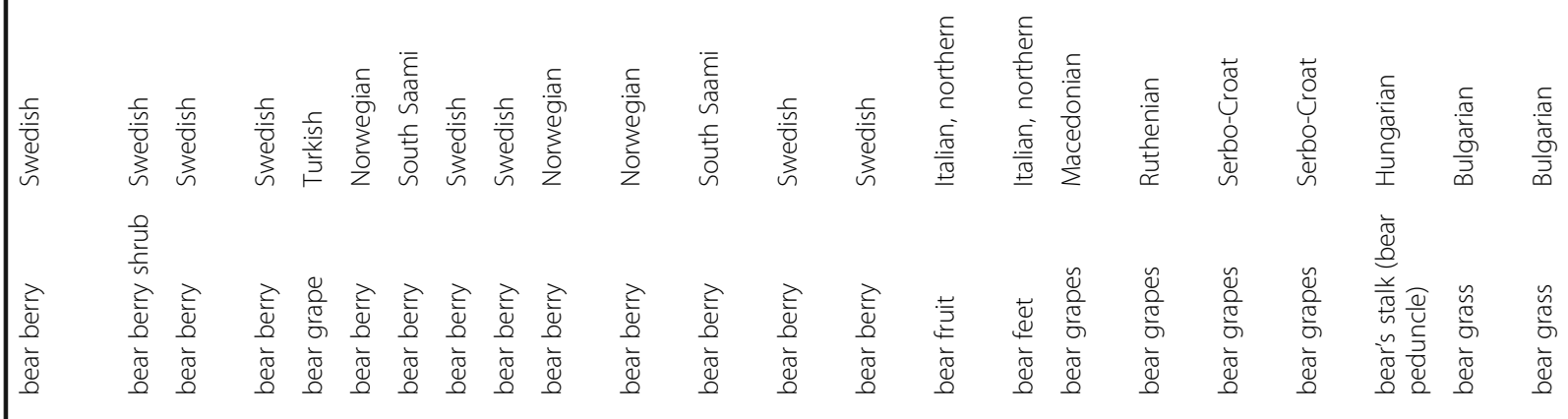

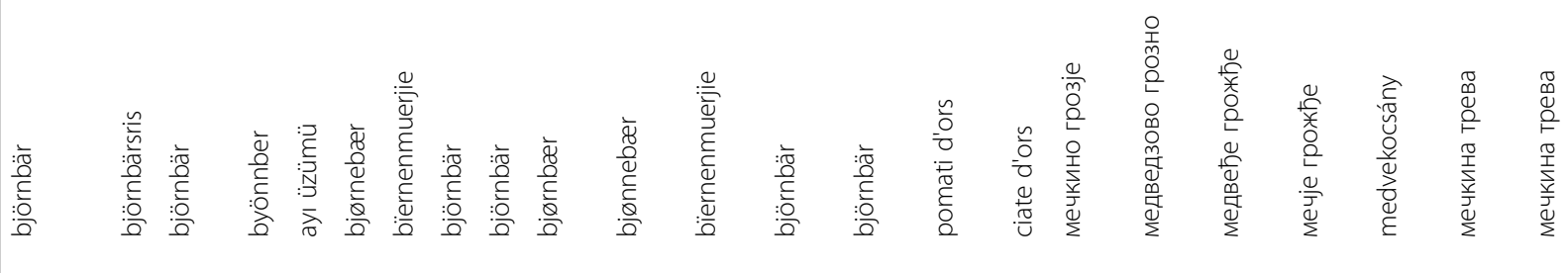

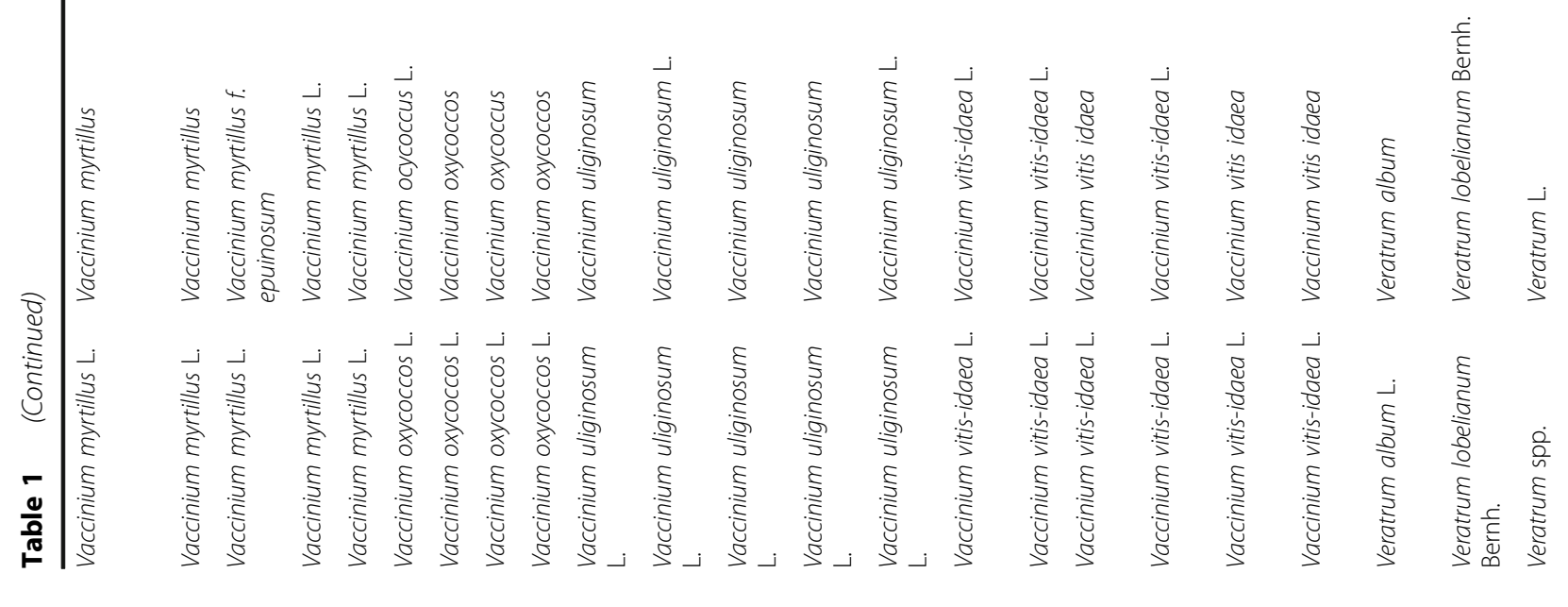




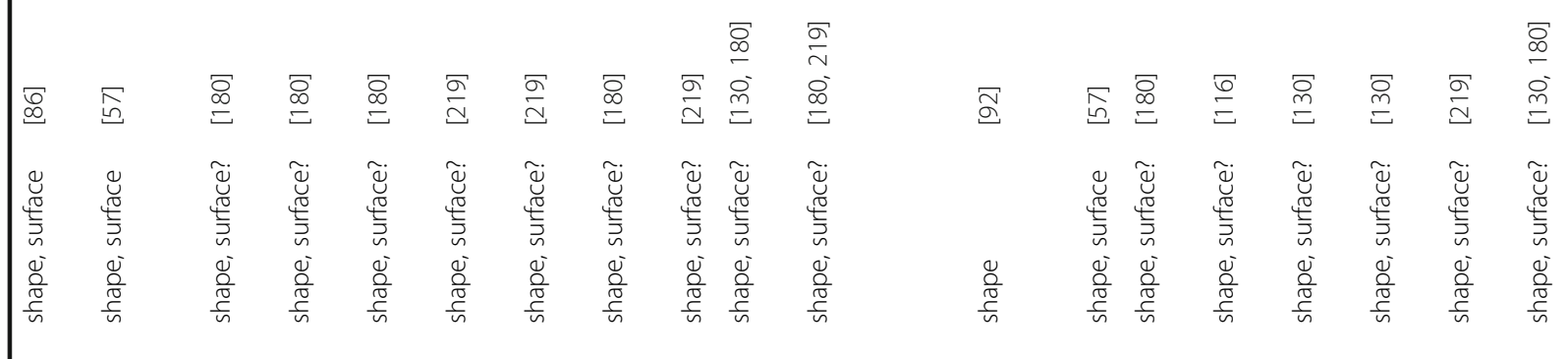

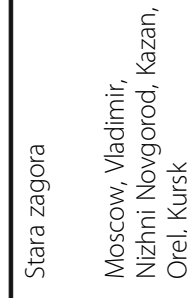

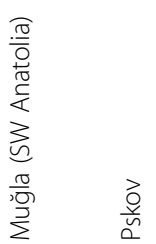

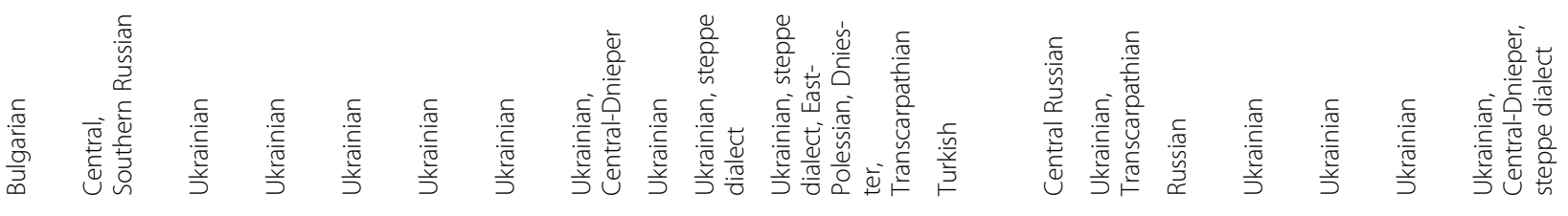

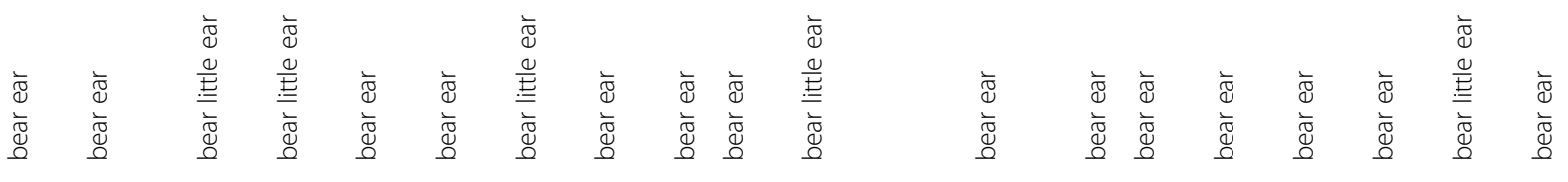

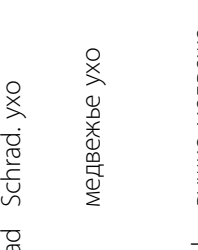

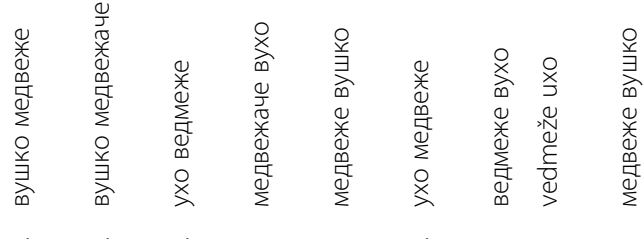

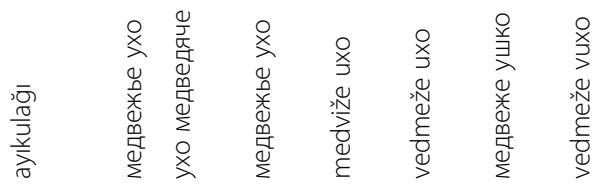

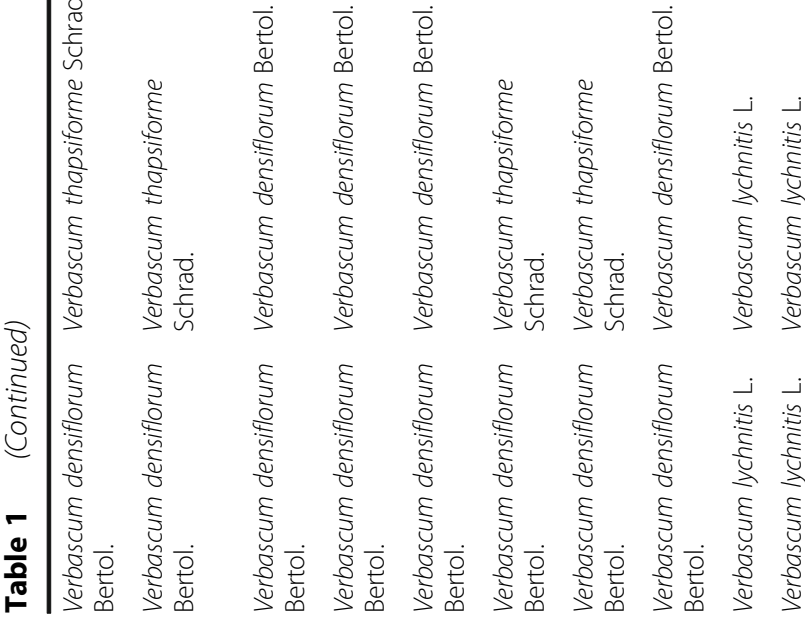

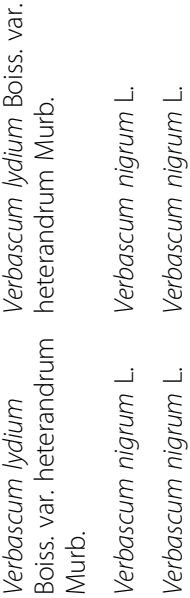

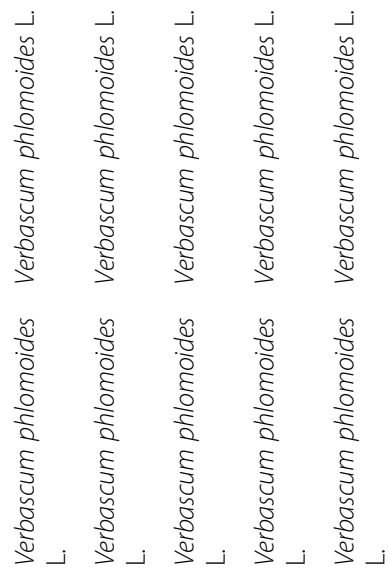




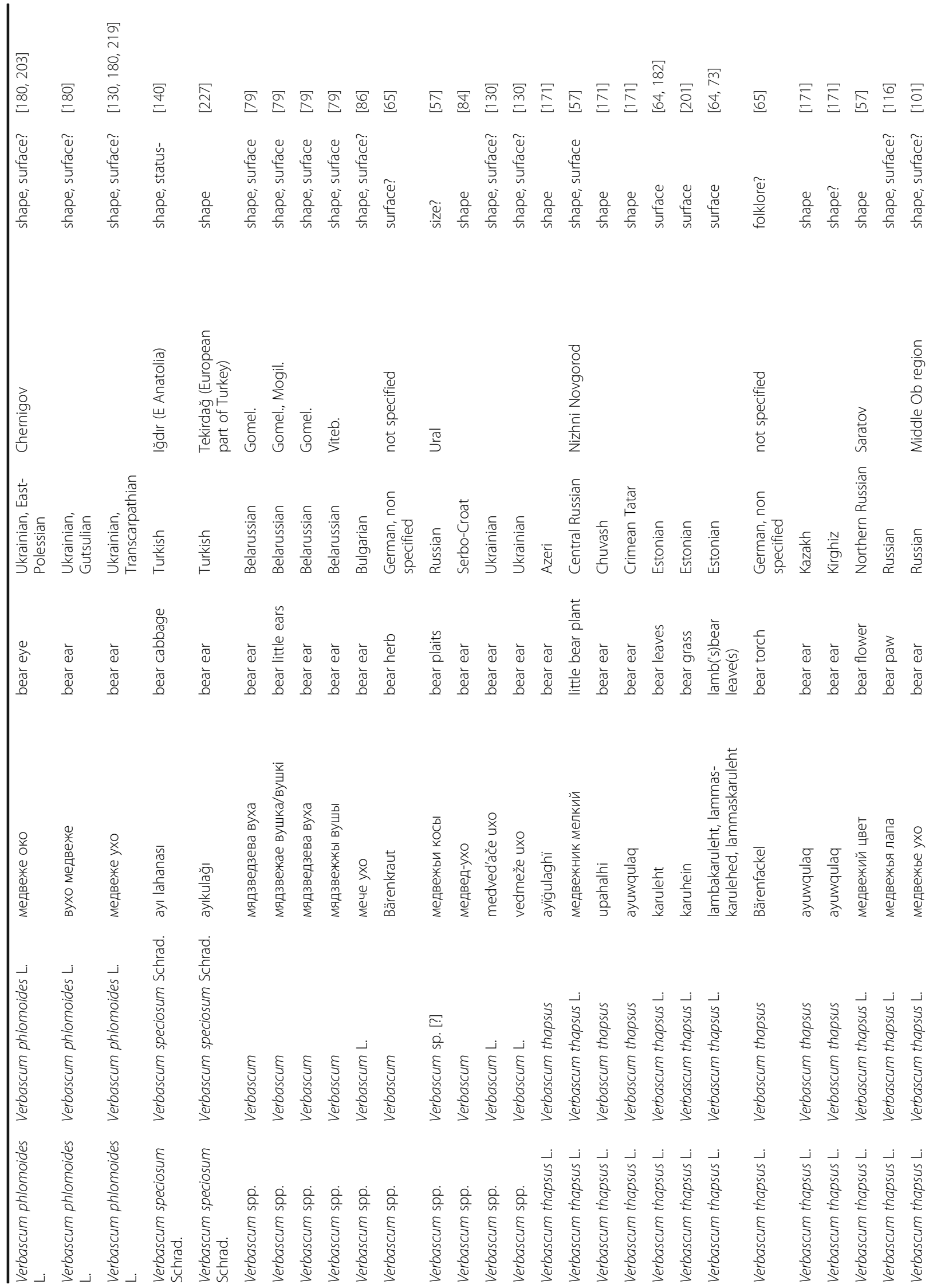




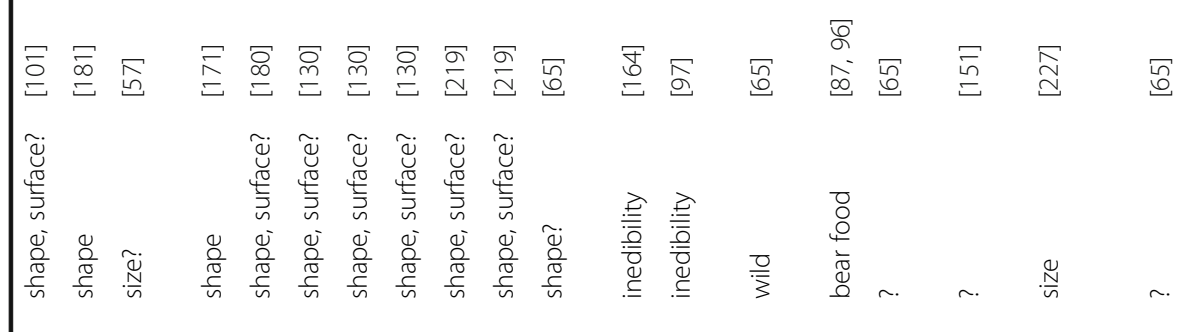

$\frac{\sqrt{\circ}}{\frac{0}{0}}$

응

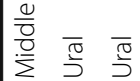

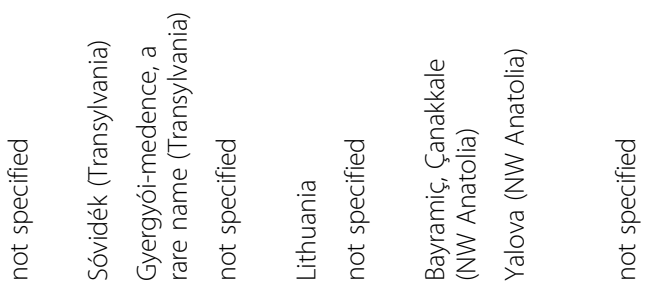

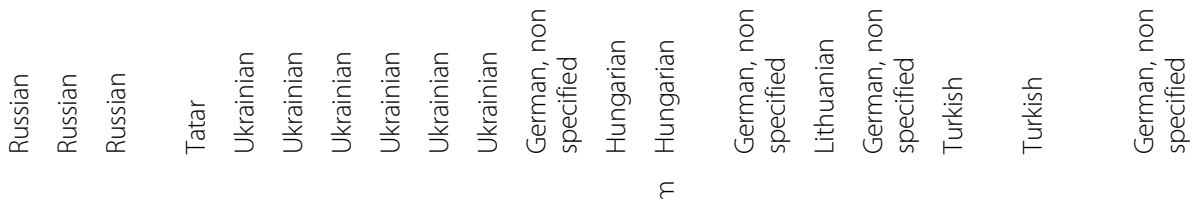

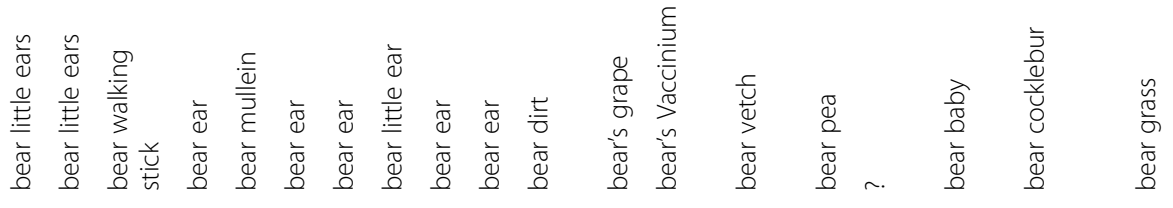

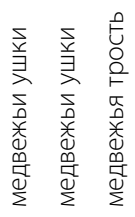
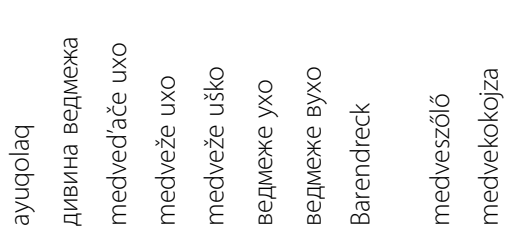

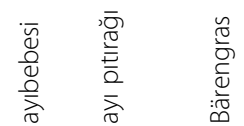

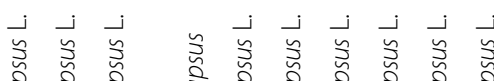

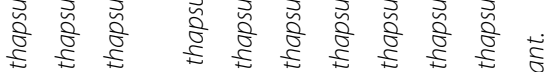

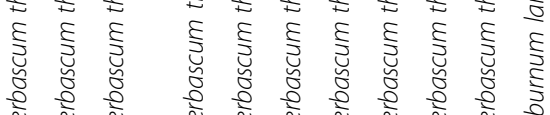

$\frac{\sqrt{2}}{8} \frac{0}{2}$

先

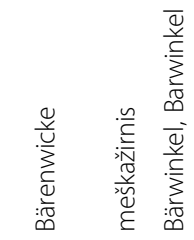

高

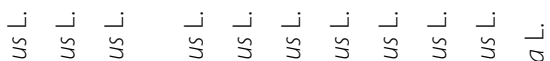
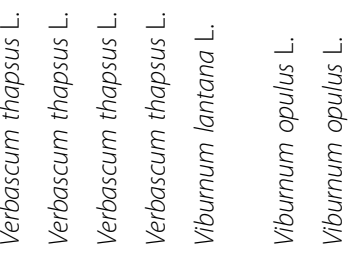

$\frac{5}{5}$
$\frac{5}{5}$
$\frac{5}{5}$

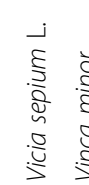

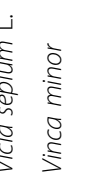

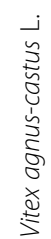

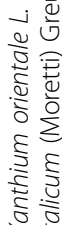

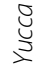


$k a h r$, and Finnish karhu) are related to each other. They are all connected with an ancient adjective (Est. kare, Fin. karhea 'rough'), which may be a very old IndoIranian loan. The words for bear coincided phonetically with another word, karune 'hairy' (where -ne is a suffix of an adjective). This word is etymologically connected with the noun karv 'hair', which is an old Baltic loan in the Baltic-Finnish languages. Gooseberry is a good example here, which in some parts of Estonia was named karusmari (Est. mari 'berry', where the 's' is a remnant of a genitive of the adjective suffix). It is of course wrong to understand this as "bear berry"; it is "hairy berry". Another Estonian name is karuohakas (Anchusa officinalis L., Cirsium lanceolatum Scop.) which have nothing to do with bear either (karu, karune and karv [48]). Still, all the aforementioned and similar cases were included into Table 1 and considered during counting the number of phytonyms.

\section{The bear as compound in Eurasian plant names}

Folk and scientific plant names have entered into intricate relationships. So, in Russia the first medicinal books (containing descriptions of various plants features) were translated in the 16th century from German and Polish, and later from Latin. Since then, a complex of naturalscience ideas (having antique, Byzantine, and WestEuropean origins) and oral folklore-mythological ideas has existed, with folk herbal books somewhere between them [49]. When the interpreters could not find an appropriate Russian equivalent, they used calques (loan translations, literal translations) or simple transliterations. This method was used also in alphabetical books (Rus. азбуковники) - the first explanatory dictionaries [50]. In Europe, "the fathers of botany" had to widen the lists of the antique authors at the expense of the local plant names [51]. Linnaeus's system influenced national nomenclatures as well. Besides, there were also foreign names borrowed by noble classes through the books, and by the peasantry - via everyday contacts with neighbouring peoples. Nowadays, strong influence is exerted by school education and media.

Children played an important role in naming of local plants. They have been very observant of details and some of these names survive for generations (cf. $[52,53])$. One example is the number of names for the sporangium of the golden maiden hair (Polytrichum commune L.) in the Germanic languages. Animal prefixes were very common (birds, mammals) and the children have seen moss as a field with crop and therefore named it crow's field, cuckoo's cereals, fox rye, etc. [54]. There was also a bear name for the Polytrichum commune L. from the northern Dalecarlia in Sweden: björnblomma ["bear flower"] [22].

While analyzing the "bear names" of plants in various languages, one may notice that the same element of a name - 'bear' - was motivated by a whole number of various plant features. Not all of them were productive to the same extent; still, the influence of each feature may be found all over the territory we studied.

It was not always possible to indicate with certainty one specific feature for each plant nomination. As it is shown in Table 2, various authors explain the bearnames of the same plant by different motivations. Very often, a name was the result of influence of a whole number of features. Moreover, one plant taxa can have various bear-phytonyms motivated by different features, for example among the 18 plants most often attributed bear-related phytonyms, we can detect between two to six different motivations, differing within the languages (Table 2). In the sections below, the names were grouped according to the "leading" assumed feature, while the other(s) was/were mentioned if relevant.

From the point of view of word-formative models, we may conclude that bear-names might be coined in the following ways: 1) "bear/she-bear" (transfer of meaning), 2) bear + a body part of the bear (e.g. ["bear's paw"]), 3) bear + a part of the plant (e.g. ["bear berry"]), 4) bear +a plant name (e.g. ["bear willow"]), 5) bear + a plant group name (e.g. ["bear tree"]), 6) bear + an object name (e.g. ["bear spindle"]).

The plant taxa having most various phytonyms within regions/languages with rich representation of bear-related phytonyms were outlined in the Table 3 . Although roughly one third of the motivations were hypothesized, the general proportion of the motivations is in large outlined in Fig. 3. The motivations below are listed based on the logical assumptions of the authors about the most popular motivations, yet the analysis proved such assumptions were incorrect.

\section{Nomination by size}

This nomination might be presumed to be the largest group, but in fact contributed only $3 \%$ of recorded or assumed motivations and some more in the combinations with other motivations. Bear names were given, as a rule, to large plants with high stalks and/or large leaves, other parts of unusually big size. Into this group such names might be included as Fin. karhunputk ["bear's thistle"], Izh. karhuntruba ["bear's pipe"] Angelica archangelica L. [55, 56]; Rus. Tobol. медвежье дерево ["bear's tree"] Asparagus [57]; Est. karuein, karuhaina, karuoblik ["bear's grass", "bear's sorrel”] Rumex crispus L. [58]. An unidentified broad-leaved meadow plant was called Rus. Perm. медвежья лапа ["bear's paw"] [57]. Turk. name ayı ardıcı ["bear's juniper"] was given to Juniperus drupacea having bigger cones than other species [59]. It seems that size was also important for Arbutus unedo L., as it is compared with grapes and strawberries, but the nominations could also be influenced by the surface of the fruit, as well as by using them as bear food. 


\section{Nomination by surface type}

Another group which assumingly could be big due to hairy look of bear and many plants, in fact contributed only $7 \%$ of the motivations. Here a good example is found in the folk names of Lycopodium spp.: in Upper Sorbian, German, Polish, Czech, Slovak, Italian, and Hungarian dialects it was compared with bear's paws, feet, legs, and hair (as well as more general words as 'herb' and '(club) moss' in Estonian and German [45, 58, 60-76];. Lycopodium was called björnmåssa ["bear moss"] in a Swedish source from 1694 [77], which is probably a translation from a German source.

Equisetum arvense in Hungarian dialects was called medveszakál(l)a ["bear's beard"], medvefarka ["bear's tail"], and medvebajusz ["bear's moustache"] [78].

\section{Nomination by form}

In this section it is possible to find several subgroups, according to the form of various parts of the plants. This is probably the reason why this group contributes to highest number of both recorded and assumed motivations (17\%). The names could be based on the appearance of:

a) large leaves of roundish shape (rather often compared to bear paws): Rus. Kostrom. медвежьи лапы ["bear's paws"], Bel. Smol., Gomel. мядзвежая лапка ["bear's little paw"], Norw. bjønnblekker ["bear's leaves"], Est. karukoll ["bear's ogre"] Caltha palustris L. [57-59, 80], Bulg. меча стъпка ["bear's step"] Tussilago farfara L. [81], Eng. bear's foot Alchemilla vulgaris L. [45].

b) spherical organs of plants (e.g. inflorescences), sometimes with thorns and prickles; could be compared with bear's head or ear: Kaz. ayïwbastiken ["bear's head"] Cirsium sp. [6], Est. karuohakad, karused uhakad, karuuhak, karuohtjas, karuõhakas ["bear's thistle", "bear's thorn"] Cirsium arvense (L.) Scop. [58], Norw. bjønnehatt ["bear's hat"] Cirsium heterophyllum L. [80], Rus. Psk. медвежник болотный ["swamp bear-plant"] Cirsium oleraceum Scop. [57]. Estonian name karune ohak for Carlina vulgaris L. literally means "bear thistle" [58]. There was also a small group of names for Centaurea spp.: Est. karukellad ["bear's bells"] [58] and Rus. медведник ["bear's plant"], медвежья лапа ["bear's paw"] [75], Turk. aylkulaği ["bear's ear"] Arctotis sp.; Aster amellus [6]. Names of mushrooms with untypical shape, for example, Liv. okš-šorrmaz ["bear's septum"] Gyromitra esculenta (Pers. ex Pers.) Fr. [81] could also be put here.

This type of nomination still stays productive; it shows, for example, in Rus. Novosib. медвежья rana ["bear's paw"] for some species of cacti; at that, the phytonym has obviously been derived recently. c) Plants with small flowers pressed in bunches, looking fluffy (often compared with ears or paws): Turk. aylkulaği ["bear's ear"] Glycyrrhiza glabra, Tat. ayu baši ["bear's head"] Trifolium sp. [6], Rus. Psk. медвежье ухо ["bear's ear"] Verbascum nigrum L. [57], Fin. karhunkukka ["bear's flower"], karhunruoho ["bear's grass"] Achillea millefolium L. [82].

As the reason for nomination was seldom indicated explicitly in sources, it seems sound to draw typological arguments. For example, a number of names for Antennaria dioica (L.) Gaertn. - Germ. Bärentatze, Bärenpratze, Cz. medvědi tlapičky, Sloven. medvedove tačice, Est. karukäpp, all literally meaning "bear's paw(s)" [58, 65, 83, 84], - finds a typological parallel in Rus. кочачьи лапки, Germ. Katzenpfoetle ["cat's paws"]. This sub-group may also be enlarged by names for Clavaria spp., compared in German, Slovenian, Bulgarian, and Serbo-Croat dialects with bear's paws, and in Slovenian also with bear's mane $[65,84,85]$.

\section{Nomination by toxicity/inedibility for humans/lower status in comparison with cultural analogues}

The toxicity along with lower status contribute in sum only $5 \%$ of records. Plants with poisonous or just inedible fruits often have the word 'bear' as the first element and the word 'berry' (or a name of some specific berry plant) as the second, as in Swedish, Norwegian, Bulgarian, Russian, Lithuanian, Estonian, and Finnish names for Paris quadrifolia L. [15, 55, 57, 58, 80, 82, 86-88]. The same may be found in many other plants names: for instance, Crataegus spp. was compared with apples in Bulgarian, and pears in Slovenian and Italian [84, 86, 89, 90]; Paeonia spp. was compared with roses in Turkish [91-94]; Ribes spp. was compared with grapes in Italian and Serbo-Croat [84, 90, 91], Corylus colurna L. - with hazelnut in Albanian [95], Lathyrus sylvestris and Vicia sepium L. was compared with peas, in Lithuanian [87, 96], Oxalis acetosella was compared with sorrel in Hungarian [97, 98] - in all these cases the abovementioned species have the 'bear' prefix. The scornful, negative character of nomination is confirmed by the fact that many of the plants analysed received other folk names with 'dog', 'wolf', 'pig', 'snake', etc. as the first component. To step beyond the scope of zoological code, the plants supposed to be unpleasant, harmful, or "not real" were also often named in ethnic terms, specific for each nominating language.

Nevertheless, as bear is taken as the dominating European animal, in Russian dialects (Novgorod, Tver, Vologda, Olonets) the bear's name was used for nomination of Boletus edulis as the best mushroom, the 


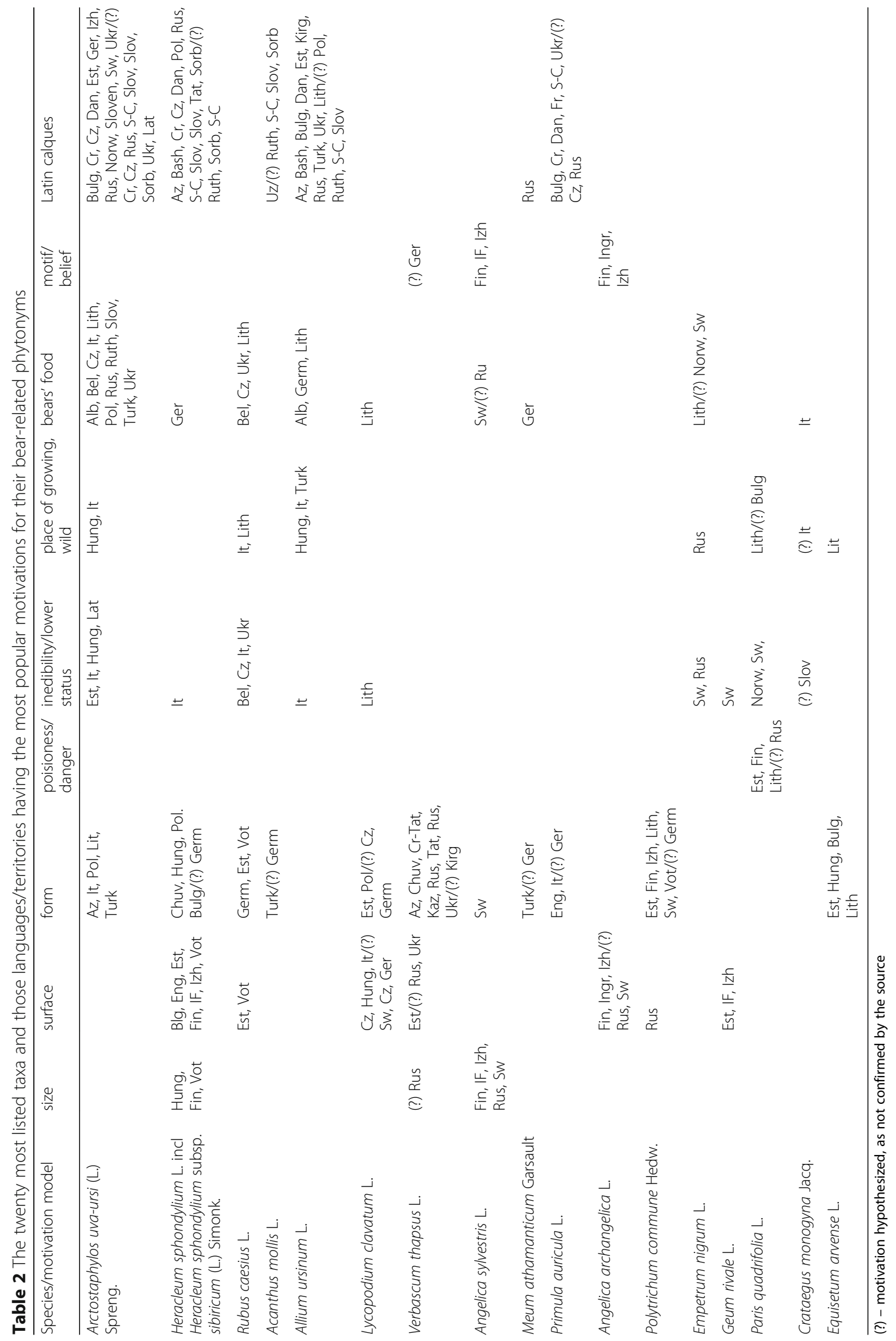


Table 3 Frequency of different phytonyms related to bears for the most named taxa in the "bear-richest" languages

\begin{tabular}{|c|c|c|c|c|c|c|c|c|c|c|c|c|c|c|c|c|c|c|c|}
\hline Taxa/languages & Est & Germ & Rus & Sw & Turk & It & Ukr & $\mathrm{S}-\mathrm{C}$ & Bulg & Sloven & $\mathrm{Cz}$ & $\mathrm{Cr}$ & Lith & Fin & Bel & Norw & Eng & Hung & Sorb \\
\hline $\begin{array}{l}\text { Heracleum sphondylium L. incl. } \\
\text { Heracleum sibiricum L. }\end{array}$ & 5 & 14 & 1 & 3 & & & & 3 & 2 & 2 & 4 & 4 & & 3 & & & 1 & 3 & 4 \\
\hline Arctostaphylos uva-ursi (L.) Spreng. & 8 & 4 & 5 & 1 & 1 & 7 & 4 & 5 & 1 & 3 & 3 & 3 & 1 & & 1 & 1 & & 1 & 2 \\
\hline Rubus caesius L. & 3 & 1 & & 3 & & 1 & 5 & & & & 3 & & 1 & & 2 & & & & \\
\hline Acanthus mollis $\mathrm{L}$. & & 1 & & & 1 & 1 & & 3 & & 1 & 2 & 3 & & & & & & & 2 \\
\hline Allium ursinum $\mathrm{L}$. & 2 & 2 & 2 & & 1 & 1 & 1 & 1 & 1 & 1 & & & 1 & & & & & 2 & \\
\hline Lycopodium clavatum L. & 1 & 5 & & 1 & & 3 & & & & & 2 & & 1 & & & & & 1 & \\
\hline Primula auricula $\mathrm{L}$. & & 2 & 1 & & & 1 & 1 & 1 & 1 & & 1 & 1 & & & & & 1 & & \\
\hline Polytrichum commune Hedw. & 1 & 1 & 1 & 2 & & & & & & & & & 1 & 1 & & 1 & & & \\
\hline Empetrum nigrum L. & 3 & & 1 & 1 & & & & & & & & & 1 & 2 & & 1 & & & \\
\hline Paris quadrifolia L. & 2 & & 1 & 1 & & & & & 2 & & & & 1 & 1 & & 1 & & & \\
\hline Arctous alpina (L.) Nied. & & 1 & & 1 & & 1 & & & & & & & & & & 1 & & & 1 \\
\hline Verbascum spp. & & 1 & 1 & & & & 1 & 1 & 1 & & & & & & 2 & & & & \\
\hline Equisetum arvense L. & 1 & & & 1 & & & & & 1 & & & & 1 & & & & & 3 & \\
\hline Heracleum spp. & 1 & & & 2 & & 1 & & & 1 & & & 1 & & & & & & & \\
\hline Calla palustris L. & 1 & & 1 & & & & & & & & & & & & 1 & 1 & 1 & & \\
\hline
\end{tabular}

Only those languages are included with at least 20 records of bear-related phytonyms, and only those taxa that have bear-related phytonyms in at least five different languages among these. The numbers show the number of different meanings bear-related phytonyms have for a specific plant in the corresponding language. In those cases where the two names have a similar meaning, but different words are used to express it, they are considered as different. Abbreviations: Est - Estonian, Germ German, Rus - Russian, Sw - Swedish, Turk - Turkish, Ukr - Ukrainian, It - Italian. S-C - Serbo-Croat, Bulg - Bulgarian, Sloven - Slovenian, Cz - Czech, Cr - Croatian, Lith - Lithuanian, Fin - Finnish, Hung - Hungarian, Norw - Norwegian, Bel - Belarussian, Eng - English, Sorb - Sorbian

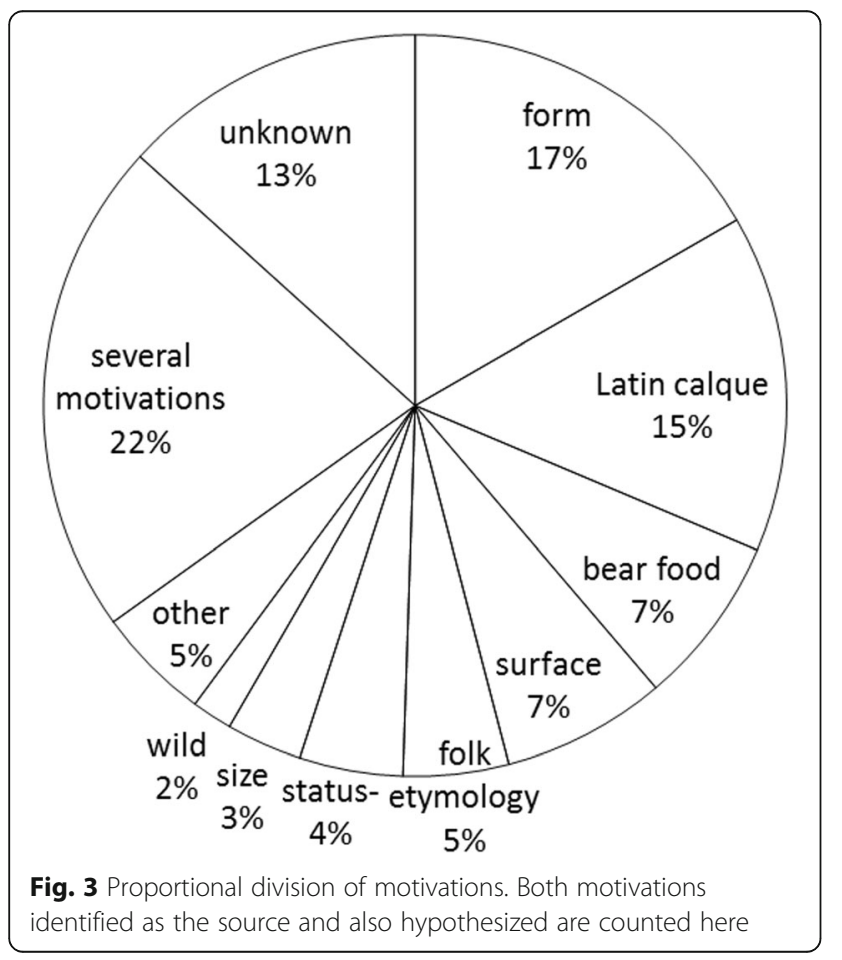

mushroom par excellence [57]. The same can be said about Hungarian folk names for $B$. edulis and other Boletus spp. in Transylvania [99, 100], and others; see the Table 1, though there is no information on motivations for corresponding cases in Turkish.

\section{Nomination by place}

Perhaps here "bear nomination" was a particular, narrower case of "wild nomination". The location in the wild is a "background feature" of the plants, growing far from the human dwelling, in the places where animals live, which serves as a basis for metaphorical alikening of a plant and an animal. Here it seems appropriate to consider a number of names for the fungus Lycoperdon spp. In this case, the ability to produce a cloud of spores caused a set of second components of phytonyms, connecting them to bathing, smoking, etc.: Germ. Bärenfurz ["bear's fart"], Bulg. мечкинь nуфети ["bear's puff"], Rus. Middle Ob медвежий дым ["bear's smoke”], медвежий табак ["bear's tobacco"], Rus. Vlad. медвежьи бани ["bear's baths"], Rus. Perm. медвежве курево ["bear's tobacco"] [65, 86, 101-103]. It is noteworthy that the same fungus was known as Fr. vesse-de-loup ["wolf's fart"], which is the literal translation from Greek. Here we see again, how a bear and a wolf, being similar in folk ideas, could be interchangeable also in folk plant nomenclature. Another example is Lychnis chalcedonica L. which has names not only Rus. Ob медвежье мыло ["bear's 
soap"], but also Rus. Ob собачье мылло, ["dogs's soap"] [101], Rus. Samar. кукушкино мыло ["cuckoo's soap"] as well a number of other names [12]. Although this motivation can be perceived as the background in many more cases, especially in case of several possible motivations, it has explicitly contributed to only $2 \%$ all records.

\section{Nomination by usage for food in bears}

Bears are typically omnivorous animals, their diet includes succulent shoots and leaves, fruits, insects, and meat [104], although recent studies have shown that carnivory is positively correlated with latitude among omnivorous mammals [105]. Omnivority of the bear gave it in many cultures the attribute of medicine animal, knowing all the plants and foods in general. As bears were often believed to have supernatural powers (due to their size and long hibernation period) people observed with great attention bear's way and the way they foraged. We can thus assume that a large proportion of bear names in plants referred to their diet. The literature on bear ecology gave us dozens of bear food plants, and some of them had bear-related names in some languages. The main example of such plants is hogweed (the genus Heracleum) reported as one of the main spring foods of the bear from many countries, e.g. the USA, China, Japan, and Poland, e.g. ([106-109], Tomasz Kozica - pers. comm.). Also "bear's garlic", Allium ursinum, was reported as important bear food in Croatia [110]. There was also an evidence from Mr. Sándor Tímár (Eastern Carpathians) of bears eating Allium ursinum and A. victorialis (Hung. vadfokhagyma, wild garlic), though the plant was not named after bear in this area: "The bear does not eat anything during winter, he licks his paws, and licks so much that by spring they are white. And then he eats first from that plant (wild garlic), in order to clean his stomach from the "deposits". $\mathrm{He}$ is such a clever animal. He searches for what he has to eat after the winter sleep". ${ }^{1}$ Bears eat a large diversity of wild fruits so it is not surprising that some of them got the names of bear berries, though it is probably impossible to say if it was because they were main fruits eaten by bears or rather those fruits which are less eaten by humans, left for the bears, like Arctostaphylos uvaursi. Bears have also been observed using plants for selfmedication, so some of the plants which are not typical bear food or do not resemble bears in any way may have acquired their names from incidents of humans observing a bear using this plant as medicine. This was the case with Ligusticum porter which was observed as being sought after by bears and was regarded as bear medicine by Native Americans [111].

It is possible to assume that some plants - their fruits, stalks or rhizomes - were eaten by bears, though dialect dictionaries seldom give explanations, and we do not always know the folk ideas behind this or that nomination. Sw. björnbär ["bear berries"] for Vaccinium myrtillus L. [15], Cz. medvédice ["bear's plant"] for Rubus caesius L. [72], Rus. медвежьи ягоды ["bear's berries"] for Rubus idaeus L. [57] seem rather reliable. Arctostaphylos alpina (L.) Spreng was also known as "bear berries" in many languages, such as N.S. guovžžamuorji, Sw. björnbär and Norw. bjønnbøer (recorded already in 1766 by Gunnerus [112]) [15].

However, not all scholars agreed that there was a necessary connection with the bear-prefix and the berries consumed by the animal. Many different kinds of berries were named björnbär locally in northern Scandinavia. Black shining fruit varieties of Vaccinium myrtillus L. and Empetrum hermaphroditum Hagerup were for instance known as björnbär "bear berries" in northern Sweden. But normal bluish-coloured fruits, although being eaten by bears, were not called "bear berries" (cf. [37]). However, in some areas the normal-coloured berries were also known as "bear berries", and known to be eaten by bears as well. Swedish plant name scholar and linguist Karl-Hampus Dahlstedt [15], who studied the north-Scandinavian berry-names in particular, stressed that the naming motif sometimes could be pejorative, for instance for Paris quadrifolia and maybe, but not necessary, for Vaccinium oxycoccos. Dahlstedt concluded that it was not easy to find one explanation for why many different kinds of berries were known as "bear berries" in northern Scandinavia [15]. Why Rubus caesius was called karuu-marja "bear-berry" in Votic is not clear either [113].

Another example of a bear food plant is Cicerbita alpina (L.) Wallr., which was known as björnmat ["bear food"] in Dalecarlia, björngräs ["bear grass"] and björnkål ["bear cabbage"] in Lapland, as well as bjønnturt ["bear plant"] and bjønnmat ["bear food"] in Norway. The plant was well-known as appreciated by bears among the peasantry in northern Scandinavia [114]. The same may be assumed about It. pan d'ors(o) ["bear's bread"] Sorbus aria L. [74, 115], and Hung. medvesaláta ["bear's lettuce"] which was a name for some woodland fringe tall herbs (Cirsium erisithales, C. oleraceum, C. rivulare, Carduus personatus) in the Eastern Carpathians.

This motivation group contributed $7 \%$ to all records and the list of plants assumingly motivated by bear food contains 66 plant taxa, among which are the taxa with several possible motivations. Very limited list of taxa detected as bear food in [105], however, only partially overlap with our extended list, as obviously not all bear food was called related to bear (like for example Populus tremula L.). Yet for example Aegopodium podagraria L., which has unknown motivation, or Taraxacum spp., Tussilago farfara L. and Trifolium spp. assumingly motivated by the form in our sources, or Urtica dioica $\mathrm{L}$. 
motivated by surface have been detected as bear food in [105], which can indicate possible earlier motivation that was later over interpreted.

\section{Nomination by folklore motif or belief}

This motivation group is not numerous (5\%). Rus. Perm. медвежий табак ["bear's tobacco"] Lycoperdon was based on the belief that "a bear, to exterminate fleas, rolls about the clearing dotted with puff-balls" [102].

Another case is presented by folk nominations of Polytrichum commune. It was called Rus. Orl. медведь ["bear"], Germ. Bärenmoos, Est. karusammal, Izh. karunsammõl, Sw. björnmossa (first recorded in 1638), Norw. bjørnemose, Liv. okš-šómal, Vot. karasamma ["bear's moss"], Lith. meškakūšis ["bear's penis"] [11, 56, $65,80,81,87,113,116]$.

Slavonic sources did not say anything about the reasons for naming, Finno-Ugrian researchers surmise form nomination type. Really, one could suppose that thick, densely growing small stalks were compared with bear fur (see Lycopodium spp. above). But there were several interpretations of this specific name on Nordic material, all of them as good as the others $[17,80]$ admitted it was not easy to interpret the name: it could be explained by the fact that the moss turned red-brown and could remind one of a bear skin, but more probable, he said, was the connection with the belief that the bears used it in their winter-home. Also the moss Rhacomitrium lanuginosum was, on the same reason, named "bear moss" in Norwegian [80].

\section{(Partial) translation from Latin}

A very important phenomenon mentioned in the beginning of the article is Latin loan translations (calques) in national phyto-taxonomies contributes $15 \%$ to all records. The most remarkable cases seem to be Arctostaphylos spp., especially Arctostaphylos uva-ursi (L.) Spreng.; its names (and over 80 of these have been recorded) have as their inner form "bear's grapes" or "bear's berries" in Albanian, Bulgarian, Czech, Danish, English, Estonian, French, German, Italian, Hungarian, Polish, Russian, Serbo-Croat, Slovenian, Turkish, and Ukrainian.

Of course, there is no guarantee that some folk names might not appear independently of learned ones, as various second components are also possible; but the greater the number of semantically identical plant names we find all over Europe, the greater the chances that they are the result of borrowing, as seems to be the case with Allium ursinum, called "bear's onion" or "bear's garlic" in Italian, German, Lithuanian, Russian, Ukrainian, Polish, Slovenian, Serbo-Croat, Bulgarian, Hungarian, Romanian, Albanian, Estonian, Turkish, Azeri, Bashkir, and Kirghiz. Böhling [117] discussed why Allium ursinum had been referred to as "bear's onion" already by the ancient Greeks. He suggested that bear in the plant epithet referred to Ursa Major (Big Dipper), - the constellation which could be seen in northern skies (other Allium species occurred in southern Europe, while A. ursinum was one of the most northerly distributed species of onions). His hypothesis is not convincing. First of all, for typological reasons, there do not seem to be any plants named after stars in any of the Germanic or Slavonic languages; the abovementioned motivation by bear food or lower status seems much more logical.

William T. Stearn [118] analysed the means of coining the medieval Latin name branca ursina ["bear's paw"] for Acanthus mollis -someone "noted a resemblance between a floral bract of Acanthus mollis and a bear's clawed paw" - and then transferred it (first in France) to Heracleum sphondylium which "has large divided leaves somewhat like those of Acanthus mollis" [118, 119]. The name branc (branque, branche) ursine Acanthus mollis, evidently, provoked the French form patte d'ours [2].

It seems that the model turned out to be rather productive, as numerous names for both plants, mostly translated as "bear's paw" (but also 'foot,' 'palm,' 'claw', 'nail', 'finger', etc.), are spread all over Europe - in Upper Sorbian, Slovak, Polish, Czech, Ruthenian (in Vojvodina), Serbian, Croat, Slovenian, Bulgarian, Russian, Romanian, French, Hungarian, English, German, Swedish, Danish, Dutch, North Saami, Votic, Tatar, Chuvash, Azeri, and Bashkir [6, $16,45,61-63,65,68,84,86,87,89,113,116,120-129]$. The model might also concern other Heracleum species; a larger group was made by folk names for Heracleum sphondylium L., mostly in Northern Europe.

In these cases we may suggest that first corresponding phytonyms penetrated to Indo-European languages, and later - very likely, through their mediation - to FinnoUgrian and Turkic ones.

A noteworthy unity of nominations was demonstrated by a number of names for Primula auricula L.: Germ. Bärenohr [65], S.-C. медвиђе ухо [84], Cz. medvédí ouško [60, 61], Eng. bear's ears, bazier, ba(i)sier [45], Rus. медвежье ушко [116], It. urie/orie d'ours, orecchio d'orso, recchietta d'urss [74], Fr. oreille d'ours [2], Ukr. medved'ače uxo [130], Bulg. мечo yxo [86]. The semantically analogous Turkish name ayı kulağ Primula auriculata, $P$. elatior subsp. pseudoelatior, $P$. longipes, $P$. veris subsp. columnae and $P$. veris subsp. macrocalyx [131]. All of them have the inner form "bear's ear(s)" - thanks to loan translation from Latin. It had a pre-Linnaean name Auricula ursi (because of its leaves which seem to be very much shaped as bear ears), which has been translated into many languages, and rendered in the medicinal and botanical literature, for instance in Germ. Berenohrlein [132], Dan. bjørnsøre [133], Sw. björnöron [134, 135]. 


\section{Several nomination types}

As it was said above, there are often reasons to assume the existence of more than one basis for nomination, and this group constituted 22\% of all motivations. Thus, Rus. Perm медвежий гриб ["bear's mushroom"] Sarcodon imbricatus (L.) P/Karst. was explained by respondents reasoning from its bitter taste; the researcher preferred to mention the size of the mushroom (reaching $30 \mathrm{~cm}$ ), as well as dark and velvety colouring of the cap [102]. But it also seems important to note that the species has greyish brittle teeth instead of gills on hymenophore which could cause comparison with bear's head or ear in Bulgarian, Czech, and Belorussian $[61,79,86]$. We have already discussed the case of Heracleum species, combining language borrowing and motivation by shape; at the same time, the plants of Heracleum genus are used by bears for food. The names for Allium ursinum also seem to combine bear-food motivation and loan translation.

\section{Motivation unknown}

Folk nomination is sometimes so doubtful and dubious that the question of motivation may remain unsolved, as it is, for example, for Alb. bar i ariut ["bear grass"] Erica herbacea L. [136], and in many other cases. Unfortunately, dialect dictionaries, while recording plant names, seldom give the motivation. This was the case in $13 \%$ of all records.

\section{Discussion and conclusions}

The material presented gives wide opportunities for linguo-geographical and ethno-cultural observations and studies. Bear phytonyms were quite widespread in some countries/languages such as Estonian (140), German (125), Russian (122), Swedish (92) and rather rare in the others - Macedonian and Ruthenian (7), Albanian and Slovak (6), Danish (5), Bashkir (4), Chuvash (3), Livonian (2) (these numbers are not absolute, as it is not always easy to differentiate phonetical variants and different phytonyms). This fact can hardly be explained only by environment differences (like for example speakers of Estonian and Livonian inhabited the same ecological niche) nor to linguistic peculiarities (as for example there are bear-rich and bear-poor languages in all main language groups) and needs deeper cultural anthropological studies combined with folklore and ethnology. A very interesting situation was observed in the Turkic languages. There are 93 recorded Turkish bear names, while the other members of the language group demonstrate a much weaker interest in this kind of nomination: 7 (in Tatar), 6 (Kazakh), 5 (Kirghiz), 2 (Turkmen and Uzbek), and 1 (in Gagauz, Uyghur, and Karachay-Balkar) - and even here, around half of these are Latin calques. However, this may, at least to certain extent, be related to the amount of the historical ethnolinguistic research done on the selected languages. Nevertheless, a rough approximation based on the mean number of names in all languages

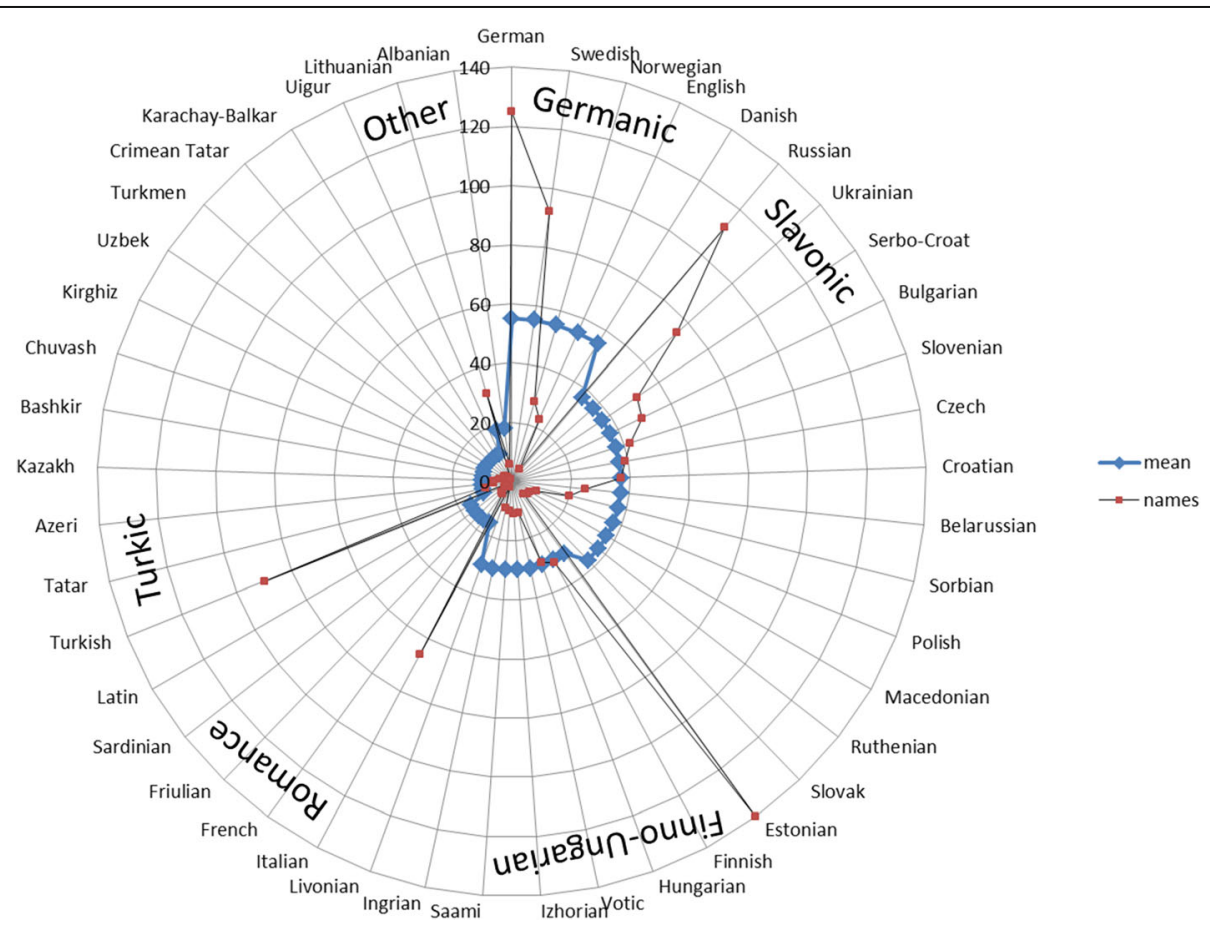

Fig. 4 Relation between language groups and the number of recorded bear-phytonyms. names - the number of recorded bear-names. mean - the mean number of names within the language group 
belonging to a language group allowed to list the language groups according to the bear-richness: Germanic, Slavonic, Finno-Ungarian, Romance, Other (Albanian and Lithuanian) and Turkic (Fig. 4). However, such approximation should not be taken too literary, as much depended on the presence of small languages and the languages we researched do not cover all languages of the groups.

From structural point of view, we may conclude that bear-names might be created according to several models: 1) bear/she-bear, 2) bear + a body part of the bear, 3) bear + a part of the plant, 4) bear + a plant name, 5) bear + a plant group name, 6) bear + an object name.

On the one hand, some plants are "champions" in bear-nomination, while others have only one or two bear names. On the other hand, some features - form and/or surface, in our case - cause a very rich pool of names, while such features as colour seem to provoke rather few associations with bear.

Some names recorded in a certain tradition may be used for explanation of dark spots in another one. For example, Serbo-Croat, Hungarian, and Turkish bearnames for Boletus spp., having no explanations inside these traditions, may be ascribed to status nomination by analogy with Russian folk names. The case of Polytrichum commune, analysed above, allows us to surmise not only form nomination in Russian, Estonian, etc. (based on Nordic explanations), but also borrowing from the Scandinavian languages, as all the names in the pool are recorded in the Northern regions of Europe.

The database we assemble is also valuable for comparative and typological studies. For example, it shows correspondence of names in Latin and in other languages and demonstrates the deep influence of Latin natural-science scholarship upon European culture. Comparing names in neighbouring cultures (e.g. Northern Russian and Estonian; Baltic and Nordic) could become a good basis for studying the question of the borrowing of plant names.

\section{Endnotes}

${ }^{1}$ Special thanks to Dániel Babai for this fieldwork evidence.

\section{Abbreviations}

\section{Geographical marks}

Gomel.: Gomelj province, Belarus; Kostrom.: Kostroma province, Russia; Middle Ob: middle part of the river Ob; Novosib: Novosibirsk area; Orl.: Orel province, Russia; Perm.: Perm province, Russia; Psk.: Pskov province, Russia; Smol.: Smolensk province, Russia; Tobol.: Tobolsk region, Russia;

Vlad.: Vladimir province, Russia

\section{Languages}

Alb.: Albanian; Az:: Azeri; Bash.: Bashkir; Bel.: Belarusian; Bulg.: Bulgarian; Chuv.: Chuvash; Cr.: Croat; Cr.-Tat:: Crimean Tatar; Cz.: Czech; Dan.: Danish; Eng.: English; Est.: Estonian; Fin.: Finnish; Fr.: French; Germ.: German; Gr.: Greek; Hung.: Hungarian; I.F.: Ingrian Finnish; It:: Italian; Izh.: Izhorian; K.-B.: Karachay-Balkar; Kaz:: Kazakh; Kirg.: Kirghiz; Lat.: Latin; Lith.: Lithuanian; Liv.: Livonian; N.S.: North Saami; Norw.: Norwegian; Pol.: Polish; Rus.: Russian;
Ruth.: Ruthenian; S.-C.: Serbo-Croat; Serb.: Serbian; Slov.: Slovak; Sloven.: Slovenian; Sorb.: Sorbian; Sw.: Swedish; Tat.: Tatar; Turk.: Turkish; Uyg.: Uyghur; Ukr.: Ukrainian; Uz.: Uzbek; Vot.: Votic Historical parishes in Estonia

Äks: Äksi; Amb: Ambla; Aud: Audru; Ha: Harjumaa; Hää: Häädemeeste; Hag: Hageri; Han: Hanila; Har: Hargla; Hel: Helme; HJn: Harju-Jaani; Hlj: Haljala; Hls: Halliste; lis: lisaku; JJn: Järva-Jaani; JMd: Järva-Madise; Jõe: Jõelähtme; Jõh: Jõhvi; Jür: Jüri; Juu: Juuru; Kaa: Kaarma; Kad: Kadrina; Käi: Käina; Kam: Kambja; Kan: Kanepi; Kei: Keila; Khk: Kihelkonna; Kod: Kodavere; Kos: Kose; Krl: Karula; Kse: Karuse; Ksi: Kursi; Kul: Kullamaa; Kuu: Kuusalu; Lai: Laiuse; Lih: Lihula; LNg: Lääne-Nigula; Lüg: Lüganuse; Mar: Martna; Mih: Mihkli; MMg: Maarja-Magdaleena; Mus: Mustjala; Nis: Nissi; Noa: Noarootsi; Nrv: Narva; Ote: Otepää; Pal: Palamuse; Pär: Pärnu; Phl: Pühalepa; PJg: Pärnu-Jaagupi; Plt: Põltsamaa; Puh: Puhja; Rap: Rapla; Rid: Ridala; Rõu: Rõuge; Saa: Saarde; Se: Setumaa; SJn: Suure-Jaani; TIn: Tallinn; Tõs: Tõstamaa; Trm: Torma; Trt: Tartu; Trv: Tarvastu; Tür: Türi; Vai: Vaivara; Var: Varbla; Vig: Vigala; Vil: Viljandi; VII: Valjala; Võn: Võnnu

\section{Acknowledgements}

We are thankful to Professor Raimo Raag, Uppsala University, for fruitful discussions. We would like to thank Professor Lars-Erik Edlund for providing us with material from the Swedish and Norwegian plant name in the Diabas Database at Umeå University. Special thanks for the opportunity to collect the data from the Turkish Plant Names' Database of Istanbul Nezahat Gökyiğit Botanic Garden (http://www.ngbb.org.tr/tr/) in 2014.

\section{Funding}

OTKA (Hungarian Scientific Research Fund) grant (PD 108534) supported work of NP; Estonian Ministry of Education and Research (EKKM14-300) supported fieldwork of RS and RK, Estonian Research Council (IUT22-5), and European Union through the European Regional Development Fund (Centre of Excellence in Estonian Studies) supported research made by RK and RS.

\section{Availability of data and materials}

The dataset supporting the conclusions of this article was included within the article as its (Table 1).

\section{Authors' contributions}

Initiation and assembling of the article (VK, IS, AP, RS, JR), "Introduction" (VK, IS, RS), identification of original and modern nomenclatures (LS), Fig. 1 (VK), all other figures and tables (RS) and various language contribution: Russian, Ukrainian, Belarusian, Serbian, Macedonian, Slovenian, Sorbian, Czech, Ruthenian (VK), Swedish (including Swedish in Finland and Estonia), Norwegian, Danish, French, Finnish, South Saami, North Saami, Livonian, Votic, Azeri, Bashkir, Chuvash, Karachay-Balkar, Kazakh, Kirghiz, Tatar, Turkish, Uyghur, Uzbek (IS), Estonian, Finnish, Ingrian Finnish, Izhorian, Votic (RS, RK), Turkish (AMGÖ), Polish, Slovak, Croatian, Ukrainian ( ŁŁ), Italian (AP, KC), German (LS, IS), Hungarian (ZM, NP), Lithuanian (DŠ), English (JR, IS), Bulgarian (DD), Albanian (AH). All authors read and approved the final manuscript.

\section{Competing interests}

The authors declare that they have no competing interests.

\section{Consent for publication}

Not applicable.

\section{Ethics approval and consent to participate}

All fieldworks followed the ethical guidances of Code of Ethics of International Society of Ethnobiology, whereas prior oral informed consent was obtained from every interviewed person. The approval of Ethic Committee for such studies is not applicable.

\section{Author details}

${ }^{1}$ Institute for Linguistic Studies, Russian Academy of Sciences, Tuchkov pereulok 9, Saint-Petersburg 199053, Russia. ${ }^{2}$ Uppsala Centre for Russian and Eurasian Studies, Uppsala University, Box 514SE-751 20 Uppsala, Sweden. ${ }^{3}$ Estonian Literary Museum, Vanemuise 42, Tartu 51003, Estonia. ${ }^{4}$ Department of Anthropology, University of Alaska Fairbanks, Fairbanks, AK 99775-7720, USA. ${ }^{5}$ Department of Pharmaceutical Botany, Faculty of Pharmacy, Ankara University, 06100 Tandoğan, Ankara, Turkey. ${ }^{6}$ University of Gastronomic 
Sciences, Piazza Vittorio Emanuele 9, 1-12042 Pollenzo/Bra, Italy. ${ }^{7}$ School of Biosciences and Veterinary Medicine, University of Camerino, Via Pontoni, 5 62032 Camerino, (MC), Italy. ${ }^{8}$ MTA Centre for Ecological Research, Institute of Ecology and Botany, Alkotmány u. 2-4, H-2163 Vácrátót, Hungary. ${ }^{9}$ Department of Pharmacognosy, University of Pécs, Rókus 2, 7624 Pécs, Hungary. ${ }^{10}$ Department of Botany; Institute of Applied Biotechnology and Basic Sciences, University of Rzeszów, Werynia 502, 36-100 Kolbuszowa, Poland. ${ }^{11}$ Institute of Biodiversity and Ecosystem Research, Bulgarian Academy of Sciences, Acad. Georgi Bonchev Str., bl. 23, Sofia 1113, Bulgaria.

${ }^{12}$ Faculty of Humanities, Vilnius University Kaunas, Sargeliai LT 60433, Žaiginio paštas, Lithuania. ${ }^{13}$ Department of Estonian and Comparative Folklore, Tartu University, Ülikooli 16, 50090 Tartu, Estonia. ${ }^{14}$ Department of Biology, University of Prishtina, St. Mother Teresa, Prishtinë, Kosovo.

\section{Received: 4 May 2016 Accepted: 19 December 2016} Published online: 21 February 2017

\section{References}

1. Thiselton-Dyer TF. The folk-lore of plants. London: Chatto \& Windus; 1889.

2. Kreiter H. Die von Tiernamen abgeleiteten Pflanzennamen im Französischen. Diss. Darmstadt: C. F. Winter; 1912.

3. Marzell H. Die Tiere in deutschen Pflanzennamen: Ein botanischer Beitrag zum deutschen Sprachschatze. Heidelberg: C. Winter; 1913.

4. Vajs N. Zoomorfski elementi u nazivima biljaka. Rasprave zavoda za jezik Instituta za filologiju i folkloristiku. 1979; IV-V:89-104.

5. Dubrovina SJ. Russkaja botanicheskaja terminologija $v$ etnolingvisticheskom osveshchenii (na materiale nazvanij rastenij, obrazovannyh ot nazvanij zhivotnyh i ptits). Kandidate dissertation. Moskva: 1991.

6. Hauenschield I. Tiermetaphorik in türksprachigen Pflanzennamen. Wiesbaden: Harrassowitz; 1996

7. Rácz J. Növénynevek enciklopédiája. Budapest: Tinta könyvkiadó; 2010.

8. Rakin AN. Nazvanija zhivotnych $v$ sostave fitonimov komi jazyka. In: Istorija, sovremennoe sostojanie, perspektivy razvitija jazykov i kuljtur finno-ugorskih narodov. Syktyvkar; 2005. p. 173-175.

9. Asseldonk van T. Herbs named after pigs. Beek-Ubbergen: Institute for Ethnobotany and Zoopharmacognosy; 2004.

10. Haber TB. Canine terms in popular names of plants. American Speech. 1963: 38:28-41.

11. Dubrovina SJ. Zoonimnye nazvanija rastenij. Voloviki i volovji travy. Acta linguistica petropolitana. Trudy Instituta Lingvisticheskih Issledovanij. 2010; $\mathrm{VI}(1): 62-68$.

12. Kolosova VB. Motivatsii slavjanskih nazvanij rastenij, obrazovannyh ot zoonimov. Traditsionnaja kuljtura. 2014;3:77-84.

13. Brodskij IV. "Medvezhij" fitonimy v finno-ugorskih jazykah. In: Leksicheskij atlas russkih narodnyh govorov 2007. St. Petersburg: Nauka; 2007. p. 85-90.

14. Kolosova VB. "Medvezhji" rastenija v russkih govorah. Russkaja rechj. 2012;5:94-7.

15. Dahlstedt KH. Det svenska Vilhelminamålet. Språkgeografiska studier över ett norrländskt nybyggarmål och dess granndialekter. Uppsala: Lundequistska; 1950.

16. Lange JE. Entire functions as limits of zero-restricted polynomials. Madison: University of Wisconsin; 1961

17. Svanberg I. De svenska växtnamnens ABC. Stockholm: Dialogos; 2009.

18. Turner W. Libellus de re Herbaria novus 1538. Edited with a translation into English by Mats Rydén, Hans Helander and Kerstin Olsson. Uppsala: Swedish Science Press; 1999.

19. Lovegrove R. Silent Fields: The long decline of a nation's wildlife. Oxford: Oxford University Press; 2008.

20. Berlin B. Ethnobiological classification. Principles of categorization of plants and animals in traditional societies. USA: Princeton Univ Press; 1992.

21. Rydén M. Vad är ett växtnamn? Några tankar kring växtnamn och växtnamnsforskning. In: Övriga namn: handlingar från NORNA:s nittonde symposium i Göteborg 4-6 december 1991. Uppsala: Norna; 1994. p. 101-11.

22. Hallowell Al. Bear ceremonialism in the Northern Hemisphere. Am Anthropol. 1926:28:1-175.

23. Zolotarev AM. The bear festival of the Olcha. Am Anthropol. 1937:39:113-30.

24. Kindaichi K, Yoshida M. The concepts behind the Ainu bear festival (Kumamatsuri). Southwest J Anthropol. 1949:5:345-50.

25. Paproth HJ. Studien über das Bärenzeremoniell I. Bärenjagdtriten und Bärenfeste bei den tungusischen Völkern. Uppsala: Religionshistoriska institutionen; 1976

26. Edsman C-M. Jägaren och makterna: samiska och finska björnceremonier. Uppsala: Dialekt- och folkminnesarkivet; 1994.
27. Gökalp A. L'ours Anatolien: un oncle bien entreprenant. Études mongoles. 1980;11:238-40.

28. Svanberg I. I samhällets utkanter: om'tattare" i Sverige. Centre for Multiethnic Research, Uppsala University, Faculty of Arts; 1987.

29. Slavjanskie drevnosti. Tt. 1-5. Moskva: Mezhdunarodnye otnoshenija; 1995-2012.

30. Brunner B. Bears: A Brief History. New Haven: Yale University Press; 2007.

31. Koby FE. L'ours des cavernes et les paléolithiques. I'Anthropologie. 1951;55:304-8.

32. Björklöf S. Björnen i markerna och kulturen. Hedemora: Gidlunds; 2010.

33. Kiersnowski R. Niedźwiedzie i ludzie w dawnych i nowszych czasach. Fakty i mity. Warszawa: Państwowy Instytut Wydawniczy; 1990

34. Pentikäinen J. Golden king of the forest: the lore of the northern bear. Helsinki: Etnika; 2007.

35. Pastoureau M. L'Ours. Histoire d'un roi déchu. Paris: Wunderkammer Verlag; 2007.

36. Medvedeva GV. Medvezhij kuljt i otrazhenie ego v ustnoj narodnij proze russkih starozhilov Vostochnoj Sibiri: semantika, sjuzhetno-motivnyj fond narrativov, nominatsii. Avtoref. diss. d.f.n. Irkutsk: 2011.

37. Levander L, Björklund S. Ordbok över folkmålen i Övre Dalarna. Vol. 1. Uppsala: Dialekt- och folkminnesarkivet; 1970.

38. Dubov IV, Sedykh VN. Svjokor-batjka govorit: k nam medveditsu vedut". Zhivaja starina. 1997:4:40-1.

39. Mollova M. Contribution aux études de tabous linguistiques. Rocznik Orientalystyszny. 1960;24:27-41.

40. Bazin L. Les noms turcs et mongols de l'ours. In: Boratav N, Dor R, Nicolas $M$, editors. Quand le crible était dans la paille. Hommage à Pertev Naili Boratav. Paris: Maisonneuve et Larose; 1978. p. 83-94.

41. Fridell S, Svanberg I. Däggdjur i svensk folklig tradition. Stockholm: Dialogos; 2007

42. Etimologicheskii slovar' slavianskikh iazykov. Vol. 18. Moscow: Nauka; 1993.

43. The Plant List. Version 1. http://www.theplantlist.org

44. Index Fungorum. Www.indexfungorum.org/.

45. Grigson G. The englishman's flora. London: Dent; 1955

46. Britten J, Holland R. English plant-names. London: Trübner for the English Dialect Society; 1878-1884

47. Wright J. The english dialect dictionary. Oxford: Henry Frowde; 1898-1905

48. Metsmägi I, Sedrik M, Soosaar SE. Eesti etümoloogiasõnaraamat. Tallinn: Eesti Keele Instituut; 2012.

49. Ippolitova AB. Russkie rukopisnye travniki XVII-XVIII vekov: Issledovanie foljklora i etnobotaniki. Moskva: Indrik; 2008

50. Kovalenko Kl. Phytonyms in Russian Manuscript Dictionaries of the 17th century. In The Second Regional Workshop on Eastern European Ethnobiology. Methodologies and methods in ethnobiology. Abstracts. 13-16. October 2011. Kira'lyre't, Hungary. Kira'lyre't. 2011:15.

51. Kupriyanov AV. Predystorija biologicheskoj sistematiki. "Narodnaja taksonomija" i razvitie predstavlenij o metode $v$ estestvennoj istorii kontsa XVI - nachala XVIII W. Sankt-Peterburg: Evropejskij universitet v Sankt-Peterburge; 2005.

52. Rydén M, Svanberg I. Kabblekans namn. Sven Bot Tidskr. 2012;106:321-2.

53. Rydén M, Svanberg I. Maskros, Taraxacum. In: Iwarsson M, Pettersson B, Tunón H, editors. Människan och floran: etnobiologi i Sverige, vol. 2. Stockholm: Wahlström \& Widstrand; 2005

54. Svanberg I. Folklig botanik. Stockholm: Dialogos; 2011

55. Koppaleva JE. Finskaja narodnaja leksika flory. Petrozavodsk: Karel'skij naučnyj centr RAN; 2007.

56. Gustav Vilbaste rahvaluulekogu. [Folklore collection of Gustav Vilbaste] 1907-1966. Estonian Folklore Archives, Estonian Literary Museum

57. Slovarj russkix narodnyx govorov. Vol. 18. Leningrad: Nauka; 1982.

58. Vilbaste $\mathrm{G}$. Eesti taimenimetused $=$ Nomina vernacula plantarum Estoniae. Eesti Teaduste Akadeemia Emakeele Seltsi toimetised, 20 (67). Tallinn: Emakeele Selts; 1993.

59. Küçüker P. Lügat-i Müşkilât-ı Eczâ'da Türkçe Bitki Adları. Uluslararası Sosyal Araştırmalar Dergisi. 2010;3(11):401-15.

60. Kosík V. Slovník lidových názvů rostlin. Praha: Školní nakladatelství pro Čechy a Moravu; 1941

61. Rystonová I. Průvodce lidovými názvy rostlin i jiných léčivých prírodnin a jejich produktů. Praha: Academia; 2007.

62. Buffa F. Vznik a vývin slovenskej botanickej nomenklatúry. K histórii slovenského odborného slovníka. Bratislava: Vydavatel'stvo Slovenskej akadémie vied; 1972.

63. Radyserb-Wjela J. Sad na štomach a keŕčkach. Časopis maćicy serbskeje. 1900;2:103-5.

64. Vallner R. Eesti rahvarohtude sõnastik: Käsiraamat apteekritele ja arstidele. Tallinn: R. Vallner; 1929

65. Marzell H. Wörterbuch der deutsche Pflanzennamen. Vol. 1-5. Leipzig: Hirzel; 1943-1972. 
66. Pabrèža J. Botanika, arba Taislius auguminis. Shenandoah, Pa.: A. M. Milukas; 1843.

67. Linde SB. Siownik języka polskiego. T. 1, cz. 1: A-F - [T. 4]: U-Z Oznaczony jako t. 6. Warszawa: Drukarnia XX. Pijarów, 1807-1814.

68. Majewski E. Słownik nazwisk zoologicznych i botanicznych polskich, zawierający ludowe oraz naukowe nazwy i synonimy polskie, używane dla zwierząt i roślin od XV-go wieku aż do chwili obecnej: źródłowo zebrane i zestawione z synonimami naukowymi łacińskiemi. Vol. 1-2. Warszawa: Nakładem prenumeratorów, skład główny w księgarni T. Paprockiego i s-ki; 1889-1898.

69. Syreniusz S. Zielnik. Kraków: 1613.

70. Knapiusz G. Thesavrvs Polonolatinograecvs Sev Promptvarivm Lingvae Latinae Et Graecae: Polonorum usui accomodatum. Quid in eo praestitum sit, in Prooemio leges. Cracoviae: Cesarus; 1621-1632.

71. Trotz MA. Nouveau dictionnaire polonois, allemand et françois: Enrichi de proverbes les plus usitez, de remarques de grammaire, de termes de medecine, de botanique, de matematique, de fortification, de marine, de chasse et des autres arts. Leipzig: Gleditsch; 1764.

72. Machek V. Česká a slovenská jména rostlin. Nakladatelství Československé Akademie Věd: Praha; 1954.

73. Wiedemann FJ. Ehstnisch-deutsches Wörterbuch. St. Petersburg: Kaiserliche Akademie der Wissenschaften; 1869.

74. Penzig O. Flora popolare italiana. Raccolta dei nomi dialettali delle principali piante indigene e coltivate in Italia. Genoa: Orto Botanico della Regia Università di Genova; 1924

75. Sella A. Flora popolare biellese. Nomi dialettali, tradizioni e usi locali. Torino: Edizioni dell'Orso; 1991.

76. Krausch HD. Wörterbuch der niedersorbischen Pflanzennamen. Manuscript. Sorbische Institute Archive. Bautzen.

77. Bromelius O. Chloris gothica, seu Catalogus stirpium circa Gothoburgum nascentium. Göteborg: J. Rahm; 1694.

78. Halászné ZK. Moldvai csángó növénynevek. Magyar csoportnyelvi dolgozatok 36. Budapest: University of Eötvös Loránd; 1987.

79. Raslinny svet: Tematychny slownik/Edited by Kuntsevich LP, Kryvitski AA. Minsk: Belaruskaja navuka; 2001

80. Høeg OA. Planter og tradisjon: Floraen i levende tale og tradisjon i Norge 1925-1973. Oslo: Universitetsforlaget; 1974.

81. Kettunen L. Livisches wörterbuch: mit grammatischer einleitung. Helsinki: Societatis Fenno-Ugricae; 1938.

82. Suhonen P. Suomalaiset kasvinnimet. Annales Botanici Societatis Zoologicae-Botanicae Finnicae Vanamo, 7(1). Helsinki: Suomalaisen Eläin- ja Kasvitieteellisen Seura; 1936.

83. Hadač E, Hadačová A, Spal J. Jména rostlin na Chodsku. Sborník Pedagogického institutu v Plzni. Jazyk a literatura. 1964; V:5-60.

84. Simonović D. Botanichki rechnik. Imena biljaka sa imenima na ruskom, angleskom, nemachkon i frantsuskom jeziku. Srpska akademija nauka. Posebna izdanja. Knj. CCCXVIII. Institut za za srpskokhvatski jezik. Knj. 3. Nauchno delo: Beograd; 1959.

85. Stranski IT. Divi i kulturni rastenija v Balgarija. Naimenovanie, razprostranenie, izpolzuvane. Rastenijata v narodnite obicha i pesni. Издателство на Българската Академия на Науките: Sofia; 1963.

86. Achtarov B. Materiali za Balgarski botanichen rechnik. Balgarska Akademia na Naukite, Pridvorna Pechatnitsa: Sofia; 1939.

87. Dagys J. Lietuviškas botanikos žodynas. Varpas: Kaunas; 1938.

88. Petkevičaitè-Bitė G. Raštai. Vilnius: Valstybinẻ grožinės literatūros leidykla; 1861-1943.

89. Karlin M. Slovenska imena naših zdravilnih rastlin. Farmacevtsko društvo Slovenije: Ljubljana; 1964

90. Manzi A. Flora popolare d'Abruzzo. Carabba Editore: Lanciano; 2001.

91. Zeynalov Y. Ilaç bitkiler tarihi gelişimi ve kullanimi. ND-Aden Yayıncılık: İstanbul; 2008.

92. Tuzlacı E. Türkiye Bitkileri Sözlüğü: Türkçe-Latince, Latince-Türkçe: Bitki Adlarının Özel Açıklamaları. Alfa: İstanbul; 2006

93. Sarıbaş, M. Bitki Adları Sözlüğü Ağaçlar-Otlar-Çalılar, Türkiye Ormancılar Derneği, Eğitim Dizisi:2, 2006

94. Karaman \$̧, Kocabaş YZ. Traditional medicinal plants of K. Maraş (Turkey). Sciences. 2001;1(3):125-8.

95. Sejdiu S. Fjalorth Etnobotanik i Shqipës. Rilindja: Prishtin; 1984

96. Grinius K. Botanikos žodynas. Manuskript. 1904.

97. Rab J. Népi növényismeret a Gyergyói-medencében. Pallas-Akadémia: Miercurea Ciuc; 2001.

98. Boris G. Népi gyógynövényismeret a székelyföldi Lövétén. BSc Thesis. Pécs: University of Pécs; 2010.
99. Pál A. Csángó növénynevek. Magyar Nyelvőr. 1909;38:379-80.

100. Szilády Z. Tájszógyűjtemény. Magyar Nyelv. 1907;3:328-33.

101. Arjanova VG. Slovar' fitonimov Srednego Priob'a. Tomsk: Izd-vo Tom. gos. ped. un-ta. 2006-2008.

102. Podjukov IA. Griby Prikamja v narodnyh nazvanijah i opisanijah: slovar' dialektnyh nazvanij gribov. Perm': OT i DO; 2012

103. Vladimirskij oblastnoj slovar': leksika prirody. Kanunova RS, Matsapaeva EM, Epifanova KV, editors. Vladimir: Vladimirskij gos. un-t. im. A.G. i N.G. Stoletovyh; 2012

104. Bojarska K, Selva N. Spatial patterns in brown bear Ursus arctos diet: the role of geographical and environmental factors. Mammal Rev. 2011; 42(2):120-43.

105. Vulla E, Hobson KA, Korsten M, Leht M, Martin AJ, Lind A, Männil P, Valdmann H, Saarma U. Carnivory is positively correlated with latitude among omnivorous mammals: evidence from brown bears, badgers and pine martens. Annales Zoologici Fennici. 2009:395-415.

106. Atwell G, Boone DL, Gustafson J, Berns VD. Brown bear summer use of alpine habitat on the Kodiak National Wildlife Refuge. In: Pelton MR, Lentifer JW, Folk GE, editors. Bears: their biology and management. Papers and proceedings of the International Conference on Bear Research and Management 4. Morges: International Union for Conservation of Nature and Natural Resources; 1980. p. 297-305.

107. Hewitt DG, Robbins CT. Estimating grizzly bear food habits from fecal analysis. Wildlife Society Bulletin. 1996:547-550.

108. McLellan BN, Hovey FW. The diet of grizzly bears in the Flathead River drainage of southeastern British Columbia. Can J Zool. 1995;73(4):704-12.

109. Schaller GB, Qitao T, Johnson KG, Xiaoming W, Heming S, Jinchu H. The feeding ecology of giant pandas and asiatic black bears in the Tangjiahe reserve, China. In: Gittleman JL, editor. Carnivore behavior, ecology, and evolution. Ithaca: Comstock; 1989. p. 212-41.

110. Kusak J, Huber D. Brown bear habitat quality in Gorski kotar, Croatia. Ursus. 1998;10:281-91.

111. Sigstedt S. How wild black bears are using Osha for medicine and helping restore a healthy global ecosystem. 2013. http://www.youtube.com/ watch? $=\mathrm{d} \times 8 \mathrm{GW} \times 65$ oM. Accessed on 6 Feb 2014

112. Gunnerus JE. Flora Norvegica observationibus praesertim oeconomicis panosque norvegici locupletata. Nidaros: Morandi; 1766.

113. Adler E, Leppik M. Vadja keele sõnaraamat, vol. 2. Eesti Teaduste Akadeemia: Tallinn; 1994.

114. Svanberg I. Järjan (Lactuca alpina) i samiskt kosthåll. In: Svanberg I, Tunón H, editors. Samisk etnobiologi: människor, djur och växer i norr. Nora: Nya Doxa; 2000. p. 259-65.

115. Pedrotti G, Bertoldi V. Nomi dialettali delle piante indigene del Trentino e della Ladinia Dolomitica presi in esame dal punto di vista della botanica della linguistica e del folklore. Monauni Editrice: Trento; 1930.

116. Annenkov NI. Botaničeskij slovar'. Sankt-Peterburg: Tipografija Imperatorskoj Akademii Nauk; 1878.

117. Böhling N. Eine Hypothese zur Ableitung des Namens "Bär"lauch. Berichte des Instituts für Landschafts- und Pflanzenökologie Universität Hohenheim. 2008; 17: 199-204.

118. Stearn WT. The tortuous tale of 'bear's breech', the puzzling bookname for "Acanthus mollis". Gard Hist. 1996;24(1):122-5.

119. Sabadosh IV. Formuvannja ukrainsjkoi botanichnoi nomenklatury. Uzhgorod: Uzhgorodsjkij derz. un-t; 1996.

120. Wjela J. Naše rostlinske mjena z přimjenami. Časopis maćicy serbskeje. 1896; 2:133-42.

121. Militzer M, Schütze T. Die Farn- und Blütenpflanzen im Kreise Bautzen. Lětopis Instituta za serbski ludospyt 1952, 1(I); 1953, 1(II). Nimorjadny zešiwk.

122. Stanko J. Antibolomenum (Antidotarium, Antibolarium) Benedicti Parthi. In: Rostafiński J, editor. Symbola ad historiam naturalem medii aevi. Kraków: Jagiellonian University; 1900 [mscr of 1472 stored in: Biblioteka Kapitulna Krakowa nr 225].

123. Karaś M, Reichan J, Okoniowa J, editors. Słownik gwar polskich. Kraków: Zakład Dialektologii Polskiej Instytutu Języka Polskiego PAN; 1977.

124. Orgelbrand M. Słownik języka polskiego. T. I-II. Wilno: Self-published; 1861.

125. Šovljanski R. Slovnik zashchiti roshlinoh i zhivotnogo stredku serbsko-ruskolatinsko-anglijski. FB Print: Novi Sad; 2010.

126. Šugar I. Hrvatski biljni imenoslov. Matica hrvatska: Zagreb; 2008.

127. Czuczor-Fogarasi J. A magyar nyelv szótára. Budapest: Magyar Tudományos Akadémia; 1867

128. Brøndegaard VJ. Folk og flora: dansk etnobotanik, vol. 3. Rosenkilde og Bagger: København; 1979. 
129. Nickel KP, Sammallahti P. Duiskka-sámi sátnegirji = Deutsch-Saamisches Wörterbuch. Davvi girji: Karasjok; 2008.

130. Makowiecki S. Słownik botaniczny łacińsko-małoruski, zebrał i ułożył w latach 1877-1932 Stefan Makowiecki. Skład główny w księgarniach Gebethnera i Wolffa: Kraków; 1936.

131. Ebcioğlu N. Bitki Adları Sözlüğü (Ingilizce-Türkçe/Türkçe-Ingilizce). Inkılap Yayınevi: İstanbul; 2009

132. Tabernaemontanus JT. New Kreuterbuch mit schönen, künstlichen und leblichen Figuren und Konterfeyten aller Gewächs der Kreuter, Wurtzeln, Blumen, Frücht, Getreyd, Gewürtz, der Bäume, Stauden und Hecken, so in Teutschen und Welschen Landen wachsen, und zu unserer Zeit gepflantz werten. Frankfurt: Nicolaus Basseus; 1588.

133. Paulli S. Flora Danica det er: Dansk Urtebog: Udi huilcken, efter hans Kongl: Mayst ... Christiani IV ... skriftlig Befalning til Facultatem Medicam, udi det Kongelig Universiteet Kiøbenhafn, icke alleeniste Urternis Historiske Beskrifvelse, Krafters oc Virkninger, med zijligste Figurer andragis: Men endocsaa Lægedomme til alle Siugdomme gafulige, korteligen oc klarligen antegnis: Saa at den er baade en Urtebog oc Lægebog. Kiöbenhafn: Melchior Martzan; 1648.

134. Franckenius J. Speculum botanicum. Upsala: Aeschillus Matthiae; 1638.

135. Rudbeckius $\mathrm{O}$. Hortus botanicus variis exoticis indigenisque plantis instructus. Upsala: Henricus Curio; 1685

136. Mitrushi I. Drurët dhe shkurret e Shqipërisë. Instituti i Shkencave: Tiranë; 1955

137. Šugar I, Gostl I, Hazler-Pilepić K. Hrvatsko biljno nazivlje: analiza hrvatskog biljnog nazivlja u djelu Liber de simplicibus Benedicti Rinij. Hrvatska sveučilišna naklada: Zagreb; 2002.

138. Šulek B. Jugoslavenski imenik bilja. Zagreb: Tiskom dioničke tiskare; 1879

139. Ahlbäck O. Ordbok över Finlands svenska folkmål, vol. 1. Forskningscentralen för de inhemska språken: Helsingfors; 1982.

140. Altundağ E. Iğdırın faydali ve zehirli bitkileri. Malatya: Medipress Matbaacılık ve Yayıncılık; 2010

141. Slovarj russkix govorov Karelii. vol. 3. Sankt-Peterburg: Izdateljstvo SanktPeterburgskogo Universiteta; 1996.

142. Atzei AD. Le piante nella tradizione popolare della Sardegna. Carlo Delfino Editore: Sassari; 2003

143. Augustin B, Jávorka S, Giovanni R, Rom P. Magyar gyógynövények. Földművelésügyi Minisztérium: Budapest; 1948.

144. Barlè J. Prinosi slovenskim nazivima bilja Zbornik za narodni život i običaje južnih Slavena. 1936, XXX(2): 181-228; 1937, XXXI(1): 169-292.

145. Baytop T. Türkiye'de Bitkilerle Tedavi Geçmişte ve Bugün, Nobel Tıp Kitabevleri; İstanbul, 1999.

146. Baytop T. Türkçe Bitki Adları Sözlüğü. Türk Dil Kurumu: Ankara; 2007.

147. Baytop T, Baytop A, Mat A, Sun S. Türkiye'de Zehirli Bitkiler, Bitki Zehirlenmeleri ve Tedavi Yöntemleri,I.Ü. Yayınları No: 3560, Eczacılık Fakültes No: 54, Gençlik Basımevi, İstanbul, 1987.

148. Bergsland K, Mattsson ML. Åarjelsaemien-daaroen baakoegærja, Sydsamisknorsk ordbok. Idut: Alta; 1993.

149. Bezlaj F. Etimološki slovar slovenskega jezika. V 5 knj. Ljubljana: Slovenska Akademija Znanosti in Umetnosti; 1977-2007.

150. Bodor K. Vadon termő gyógynövények. Mezőgazdasági és Erdészeti Könyvkiadó: Bucharest; 1963.

151. Bulut GE: Bayramiç (Çanakkale) Yöresinde Etnobotanik Araştırmalar. Marmara Üniversitesi Sağlık Bilimleri Enstitüsü, Farmasötik Botanik Anabilim Dalı, Doktora Tezi İstanbul: 2008

152. Çimen A. Uzundere ve Çevresinin Tıbbi Aromatik Bitkileri Artvin Çoruh Üniversitesi Fen Bölümleri Enstitüsü Orman Mühendisliği Anabilim Dalı, Yüksek Lisans Tezi, Artvin, 2009.

153. Coassini Lokar L, Poldini L, Angeloni Rossi G. Appunti di etnobotanica del Friuli-Venezia Giulia. Gortania-Atti del Museo Friulano di Storia Naturale. 1983; 4:101-152

154. Fridner G. Folkliga växtnamn i Västerbotten. Språk- och folkminnes institutet: Umeå; 1999.

155. Diabas: North Germanic Geolexical Database, Department of Language Studies, Umeå University, Umeå, Sweden.

156. Discorsi di MPA: Matthioli (Sinj) \& Herbario Nuovo di Castore Durante. 1717 (na Visovcu).

157. Dobrovol'skij VN. Smolenskij oblastnoj slovar'. Tipografija P.A. Silina: Smolensk; 1914.

158. Durmuşkahya C. Ege Bölgesi'nde Doğal Yayılış Gösteren Ağaçlar ve Çalılar Fotoğraflı Bitki Tanıma Kılavuzu. T.C. Çevre ve Orman Bakanlığı Yayını: Ankara; 2006.
159. Ekim T. A Botanical Field Trip to Geyik Dağı. Karaca Arboretum Mag. 1999; 5:16.

160. Çubukçu B, Sarıyar G, Meriçli AH, Sütlüpınar N, Mat A, Meriçli F. Fitoterapi Yardımcı Ders Kitabı. I.Ü. Basım ve Yayınevi Müdürlüğü; İstanbul; 2002.

161. Frendl K. Népi növényismeret, nép humán- és állatgyógyászati adatok gyûjtése a Székelyföldön (Kápolnásfalu, Szentegyháza). Mosonmagyaróvár: University of West-Hungary; 2001. MSc thesis.

162. Gilić S. Rječnik bilja. Građa za hrvatsku fitonimiju. Stanislav Gilić - Erebia Gorana: Rijeka; 2004

163. Gornitskij KS. Spisok russkih i nemnogih inorodcheskih nazvanij rastenij. Har'kov: 1890

164. Gub J. Erdő-mező növényei a Sóvidéken. Firtos Culture Institute: Corund; 1996.

165. Günbatan T. Çamlıdere (Ankara) Halk Illaçları, Gazi Üniversitesi Sağlık Bilimleri Enstitüsü, Farmakognozi Anabilim Dalı. Ankara: Subat; 2011.

166. Kovács A. Járok-kelek gyöngyharmaton... növény- és állatnevek a FelsőSzigetköz tájnyelvében. Mosonmagyaróvár: Mosonmagyaróvári Helytörténeti Füzetek 6; 1987

167. Herman O. A magyar pásztorok nyelvkincse. Budapest: Kir. Magyar Természettudományi Társulat; 1914

168. Penavin O. Bácskai és bánáti (népi) növénynevek. Forum Könyvkiadó: Újvidék; 2002.

169. Harvey JH. Osmanlı ve islam dünyasinda bahçecilik ve bitkiler. Bağbahçe Dergisi. 2008;15:30-2.

170. Hasselbrink G. Südlappisches Wörterbuch I-III. Uppsala: AB Lundequistska; 1981-1985.

171. Hauenschield I. Türksprachige Volksnamen für Kräuter und Stauden mit den deutschen, englischen und russischen Bezeichnungen. Otto Harrassowitz: Wiesbaden; 1989.

172. Heinsoo H. Kuidas vadjalased mäletavad taimede nimetusi. Õdagumeresoomõ kodo, Võro Instituudi toimõtisõq 20. 2007:33-53.

173. Hupel AW. Topographische Nachrichtenvon Lief- und Ehstland: nebst vollständigen register über alle drey bände, vol. 2. Riga: J. F. Hartknoch; 1777.

174. Eesti murrete sõnaraamat. II köide. 9. vihik, Kandetagune - kats/Eesti Teaduste Akadeemia, Eesti Keele Instituut. Tallinn: Eesti Keele Instituut; 1999.

175. İkinci N. Türkiye'nin zambak Türleri. Bağbahçe Dergisi. 2006:4:16-8.

176. Jávorka S. A magyar flóra: Magyarország virágos és edényes virágtalan növényeinek meghatározó kézikönyve I-II. Budapest: Studium; 1924-1925.

177. Kalwaitis W. Lietuwiszkų Wardų Klètelè. Spausdinta O.v. Mauderodés: Tilžèje; 1910.

178. Kızılarslan Ç. "İzmit Körfezi'nin Güney Kesiminde Etnobotanik Bir Araştırma" Istanbul Üniversitesi Sağlık Bilimleri Enstitüsü, Farmasötik Botanik Anabilim Dalı, Yüksek Lisans Tezi. İstanbul. 2008.

179. Kitanov B. Narodni imena na rastenijata ot Iztochna Makedonija. Spisanie na Balgarskata Akademija na naukite i izkustvata. 1943; LXVIII:81-107.

180. Kobiv J. Slovnyk ukrainsjkih naukovyh i narodnyh nazv sudynnyh roslyn. Kyiv: Naukova dumka; 2004.

181. Konovalova NI. Slovarj narodnyh nazvanij rastenij Urala. Ekaterinburg: 2000

182. Kunder J. Looduse õpetus. 2. raamat: Taimede riik. Tartu: Schnakenburg; 1881

183. Wiedemann FJ, Weber E. Beschreibung der phanerogamischen Gewächse Ehst-, Liv- und Curlands mit möglichst genauer Angabeder Fundorte und der geographischen Verbreitungnebst Andeutung über den Gebrauch in medicinischer, technischer und öconomischer Beziehung. Reval: F. Kluge; 1852.

184. Kõrv J. Tarwiline õpetus maja-aia pidamisest. Tartu: K. Mattiesen; 1881.

185. Spuhl-Rotalia J. Kodumaa marjad. 2., parand. ja täiend. tr. Haapsalu: M. Tamverk; 1912.

186. Lajnert J. Rostlinske mjena. Serbske. Nemske. Łaćanske. Berlin: Volk und wissen Volkseigener Verlag; 1954.

187. Lange J. Ordbog over Danmarksplantenavne. Vol. 1-3. København: Ejnar Munksgaard; 1959-1960.

188. László I. Válaszok a szerkesztőség kérdéseire 5. Gyümölcsnevek. Magyar Nyelvőr. 1901;30:347-51.

189. Drąsutavičius K. Botanikos terminų ir augalų vardų lietuviškai lotyniškas žodynèlis. Manuskript. 1902.

190. Juška A. Litovskij slovar' A. Juškeviča s' tolkovaniem' slov' na russkom' i polskom' jazykach'. T. I-II. Ross. Akademija Nauk: St. Petersburg/Petrograd; 1922.

191. Ivinskis L. Kalendorius arba metskaitlius. Vilnius: J. Zavadskio spaustuvè 1946-1979.

192. Lietuviu kalbos žodynas, I-XX t. Vilnius: Lietuviu kalbos institutas: 1941-2002.

193. Urbonas V. Lietuvos grybų atlasas. Lututė: Kaunas; 2007. http://www. hardydrew.com/label/VideoHive+1582712+Black+Bear+Eating+Berries+6+|+ Stock+Footage+download.html. 
194. Lauchyute YA. Slovarj baltizmov v slavyanskih yazykah. Nauka. Leningradskoe otdelenie: Leningrad; 1982.

195. Łuczaj Ł, Zovko-Končić M, Miličević T, Dolina K, Pandža M. Wild vegetable mixes sold in the markets of Dalmatia (southern Croatia). J Ethnobiol Ethnomed. 2013;9:1

196. Wróbel H. Związki staroczesko-staropolskie w terminologii botanicznej. Zeszyty Naukowe WSP w Katowicach. Sekcja Językoznawcza. Katowice; 1962:105-137.

197. Materyjaly aguljnaslavjanskaga lingvistichnaga atlasa. Raslinny svet. Minsk: Prava i ekanomika; 2009.

198. Mart S, Türkmen N. Bahçe ve Hasanbeyli (Osmaniye) bölgesinin etnobotanik kültürü. Ot Sistematik Botanik Dergisi. 2008;15(2):137-50.

199. Mataracı T. Ardıç. Bağbahçe Dergisi. 2010;32:28-31.

200. Moesz G. Székely és csángó növénynevek. Magyar Nyelv. 1908;4:29-34.

201. Mändmets J. Korjakem rahva suust lillede ja rohtude nimesid! Eesti Kirjandus. 1916:119-120.

202. Omeljkovetsj RS. Atlas zahidnopolisjkih nazv likarskyh roslyn. Lutsjk: RW "Vezha" Volynsjkoho derzh. un-tu im. Lesi Ukrainki; 2003.

203. Osadcha-Janata N. Ukrainsjki narodni nazvy roslin. New-York: UVAN; 1973.

204. Fancsali I. Reevaluarea actiunii plantelor medicinale folosite in etnomedicina din bazinul superior al Trotusului (Ghimes). MSc thesis: University of Medicine and Pharmacy, Tirgu Mures; 2010.

205. Hadač E. Lidové názvy vyšších rostlin plzeňského okresu. Sborník Vyšší Pedagogického Institutu v Plzni. 1958; IS:37-70.

206. Servettaz O, Rando MT. Ricerche sull'uso delle piante medicinali in Val Rendena. Webbia: Journal of Plant Taxonomy and Geography. 1979;33(2):511-29.

207. Pellegrini GB, Rossi GB. Flora popolare agordina (Contributo allo studio del lessico della Val Cordevole). Francolini: Firenze; 1964.

208. Pieroni A, Nedelcheva A, Hadjari A, Mustafa B, Scaltriti G, Cianfaglione K, Quave CL. Local knowledge on food plants and "domestic" medicines in the mountain villages of Peshkopia, Eastern Albania. J Mt Sci. 2014;11:180-94.

209. Praprotnik N. Henrik Freyer in njegov seznam slovanskih rastlinskih imen (Verzeichniß navischen Pflanzen-Namen) iz leta 1836. Scopolia. 2007;61:1-99.

210. Qafzezi N. Fjalor i bijqësisë shqip-latinisht-italisht-rusisht. Tiranë: Shtëpia Botuese 8 Nentori; 1978.

211. Demiri M. Përcaktues bimësh (Flora e vogël e Shqipërisë). Shtëpia Botuese e Librit Shkollor: Tiranë; 1979.

212. Qvigstad J. Lappiske Plantenavne. Nyt Magazin for Naturviidenskaberne. 1901;39:303-26.

213. Molnár Z, Babai D. Népi növényzetismeret Gyimesben I. Botanikai Közlemények. 2009:96(1-2):117-43.

214. Saareste $A$. Eesti keele mõisteline sõnaraamat = Dictionnaire analogique $d e$ la langue estonienne: avec un index pourvu des traductions en français. III. Stocholm: Vaba Eesti; 1962

215. Sabadosh IV. Atlas botanichnoi leksiki ukrainsjkoi movy. Uzhgorodsjkij derz. un-t: Uzhgorod; 1999.

216. Schlosser JK. Vrtna cvjetana ili opis... Manusript stored in the library of the faculty of Botany, University of Zagreb; 1870-1872.

217. Schlosser JK, Vukotinović L. Flora croatica. Zagreb: JAZU; 1869.

218. Skalozubov NL. Botanicheskij slovar'. Narodnye nazvanija rastenij Toboljskoj gubernii, dikorastushchih i nekotoryh kuljturnyh. Ezhegodnik Toboljskogo Gubernskogo Muzeja. 1911;XXl:1-86. Toboljsk: 1913.

219. Smyk GK. Korysni ta ridkisni roslyny Ukrainy. Kyiv: "Ukrainsjka radjansjka entsyklopedija" im. M.P. Bazhana; 1991.

220. Söderström S. Arnäsmalet. Uppsala: Dialekt- och folkminnesarkivet; 1994

221. Szánthó G. Válaszok a szerkesztőség kérdéseire 2. Bóka Magyar Nyelvőr. 1902;31:214-20.

222. Spólnik A. Nazwy polskich roślin do XVIII wieku. Ossolineum: WrocławWarszawa-Kraków-Gdańsk-Łódź; 1990.

223. Steensland L. Älvdalska växtnamn förr och nu. Dialekt- och folkminnesarkivet: Uppsala; 1994

224. Svahn M. Finnskägg, tåtel och sia: om folkliga namn på gräs. Dialekt-, ortnamns- och folkminnesarkivet: Umeå; 1991.

225. Janda J. Počela botanike za više nazrede srednjih učilišta. Zagreb: Zemaljska naklada; 1878.

226. Tuncer H (çev.). Yabani Bitkiler Sözlüğü. Hayati Zade Mustafa Feyzi Efendi (IV. Sultan Mehmet zamanı). I. Cilt. T.C. Gıda Tarım ve Hayvancılık Bakanlığı Basın Yayın ve Halkla Illişkiler Dair. Başk. Ankara: Atak Matbaası; 1978

227. Tuzlacı E. Türkiye Bitkileri Sözlüğü. Genişletilmiş 2. Baskı. Alfa Basım Yayım Dağıtım Ltd.Şti; İstanbul; 2011.

228. Vajs N. Hrvatska povijesna fitonimija. Institut za hrvatski jezik i jezikoslovlje: Zagreb; 2003
229. Horvatić S. Ilustrirani bilinar. Školska knjiga: Zagreb; 1954.

230. Hirc D. Slike iz hrvatske cvjetane. Zagreb: Hrvatski pedagogijsko-kniježevni sbor; 1880.

231. Korlević A. Dra Vjekoslava Pokornoga BOTANIKA za niže razrade srednjih učilišta. Zagreb; 1911.

232. Waniakowa J. Polskie gwarowy nazwy dziko rosnących roślin zielnych na tle słowiańskim. Zagadnienia ogólne. Wydawnictwo Uniwersytetu Jagiellońskiego: Kraków; 2012

233. Aasen. Norske Plantenavne. Budstikken. 1860:9-37.

234. Bartoš F. Dialektický slovník Moravský. Praha: Č. l; 1903.

235. Başer KHC. Sarımsak (Allium sativum L.) Bağbahçe. 2013:48:30-31.

236. Wiedemann FJ, Hurt J: Ehstnisch-deutsches Wörterbuch. St. Peterburg: Kaiserliche Akademie der Wissenschaften; 1893.

237. Sõukand R, Raivo K: Historistlik Eesti Rahvameditsiini Botaaniline Andmebaas (HERBA). 2008. http://herba.folklore.ee.

238. Kalle R, Sõukand R. Current and Remembered Past Uses of Wild Food Plants in Saaremaa, Estonia: Changes in the Context of Unlearning Debt. Economic Botany 2016;70:235-253

239. Vocabolario italiano-illirico. Manuscript (appr. 18th century) deposited in Dubrovnik in the Monastery of Minor Friar Franiscans, signature no. 19.

240. Özhatay N, Eminağaoğlu Ö, Esen S: Karlı Yaylaların Sakı Bahçesi“Ardahan'ın Doğal Çiçekleri" TC Ardahan Valiliği, III Çevre ve Orman Müdürlüğü, Promat Basin Yayin San. ve Tic.A.Ş.; İstanbul; 2010.

241. Özhatay N, Ok T: Doğanın Penceresinden Kahramanmaraş. Kahramanmaraş Valiliği, II Çevre ve Orman Müdürlüğü, İstanbul; 2008

242. Özuslu E, İskender E: Gaziantep'in (Soğanlı) Geofit Bitkileri. Gaziantep: Şehitkamil Belediyesi Kültür Yayını; 2010.

243. Ülken MC: Dr. Ot :"Ege ve Akdeniz Bölgelerinin Sifalı Bitkileri". Altın Kitaplar Yayınevi; İstanbul; 1999.

244. Šulek B: Biljarstvo. Uputa u poznavanje bilja. Dio 1. Beč: Albrecht; 1856.

245. Šulek B: Biljarstvo. Uputa u poznavanje bilja. Dio 2. Zagreb: Brzotisak Karla Albrechta; 1859

\section{Submit your next manuscript to BioMed Central and we will help you at every step:}

- We accept pre-submission inquiries

- Our selector tool helps you to find the most relevant journal

- We provide round the clock customer support

- Convenient online submission

- Thorough peer review

- Inclusion in PubMed and all major indexing services

- Maximum visibility for your research

Submit your manuscript at www.biomedcentral.com/submit
) Biomed Central 